\title{
ANGLES AND DAEMONS: \\ SPIN CORRELATIONS AT THE LHC
}

\author{
by \\ Nhan V. Tran \\ A dissertation submitted to The Johns Hopkins University in conformity with the \\ requirements for the degree of Doctor of Philosophy. \\ Baltimore, Maryland \\ September, 2011 \\ (C) Nhan V. Tran 2011 \\ All rights reserved
}




\section{Abstract}

The Large Hadron Collider has recently started collecting data, opening a new energy regime. This will allow us to probe further than ever before many of the current mysteries of the field. New physics beyond the Standard Model, the field's current paradigm, could manifest itself via new particles. In addition, the Higgs boson, hypothesized as a consequence of electroweak symmetry breaking, remains undiscovered. At the time of discovery, the properties of such particles will be unknown. In order to understand the nature of any new physics, it will be important to understand the properties of that new particle. Methods are presented for measuring its spin, parity and coupling to the Standard Model particles. These methods are implemented at the Compact Muon Solenoid experiment and an analysis is presented with the data collected during 2010 and 2011 running at the Large Hadron Collider. An application of these techniques is used to make a measurement of the weak mixing angle. A current status of the search for the Higgs boson is also presented.

Primary Reader: Andrei Gritsan

Secondary Reader: Bruce Barnett 


\section{Dedication}

Ross: You had a rough day, huh?"

Rachel: Yeah, just so weird seeing him like that, you know? I mean he is a doctor. You don't expect doctors to get sick.

Ross: But we do...

- Friends

To everyone who supported, encouraged and laughed with me along the way... 


\section{Contents}

Abstract $\quad$ ii

List of Tables vii

List of Figures $\quad$ ix

1 Introduction 1

1.1 The Standard Model and the $\mathrm{LHC}$ era $\ldots \ldots \ldots \ldots$

1.2 Beyond the Standard Model .................. 5

1.3 Angles and Daemons . . . . . . . . . . . . . . 10

2 Physics foundations $\quad 12$

2.1 Angular Momentum . . . . . . . . . . . . . . . . . 13

2.2 Helicity formalism . . . . . . . . . . . . . . . . 18

2.3 The Standard Model, redux . . . . . . . . . . . . . . . . . . 21

3 The LHC and the CMS detector 31 


\section{CONTENTS}

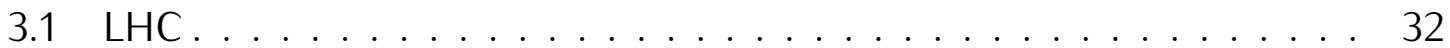

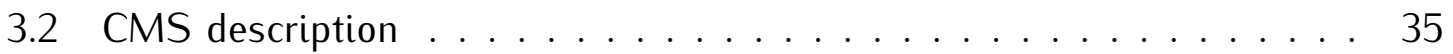

3.3 Commissioning and Tracker Alignment . . . . . . . . . . . . 45

4 Kinematics of Generic Resonances $\quad 63$

4.1 Resonances at the $\mathrm{LHC} \ldots \ldots \ldots \ldots$

4.2 General interactions with SM fields . . . . . . . . . . . . . . 68

4.3 Helicity amplitudes . . . . . . . . . . . . . . . 73

4.4 Angular distributions of spin- $J$ resonances $\ldots \ldots \ldots$. . . . . . . 77

4.5 Applications at the $\mathrm{LHC} \ldots \ldots \ldots \ldots$

5 Measurement of the Weak Mixing Angle $\quad 97$

5.1 Analytical Parameterization of the Drell-Yan Process . . . . . . . . . 100

5.2 Event Reconstruction . . . . . . . . . . . . . . . . . . 114

5.3 Analysis Strategy . . . . . . . . . . . . . . . . . . . 119

5.4 Systematic Uncertainties . . . . . . . . . . . . . . . . . 128

5.5 Results and Discussion . . . . . . . . . . . . . . . . . 144

6 Study of resonances in $X \rightarrow Z Z \rightarrow 4 \ell \quad 149$

6.1 Spin and $C P$ determination of resonances in $X \rightarrow Z Z \rightarrow 4 l \ldots 150$

6.2 Search for the Higgs in $H \rightarrow Z Z \rightarrow 4 \ell \ldots \ldots \ldots \ldots$

7 Conclusion and Outlook 198 


\section{CONTENTS}

$\begin{array}{ll}\text { Acknowledgements } & 202\end{array}$

$\begin{array}{ll}\text { Bibliography } & 206\end{array}$ 


\section{List of Tables}

2.1 Electroweak quantum numbers . . . . . . . . . . . . . 27

3.1 LHC nominal and current parameters . . . . . . . . . . . . 34

3.2 RMS of the distribution of the median of the residuals (DMR) . . . 53

4.1 Summary of resonance production and decay at the LHC . . . . . 67

4.2 Summary of allowed helicity states for spin-zero, -one and -two resonances. . . . . . . . . . . . . . . . . . . . . 92

5.1 Relative values of standard model neutral current couplings to fermions [61]. . . . . . . . . . . . . . . . . . . . . 102

5.2 Systematic uncertainties and corrections to the fit values in the mea-

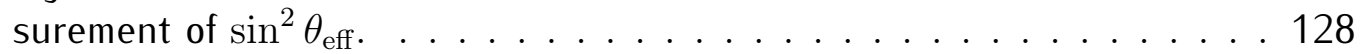

5.3 Effective values of $\sin ^{2} \theta_{\text {eff }}$ for different fermion couplings to the $Z$ boson. 129

5.4 Results of the MC cross-fits using Horace $(\alpha)$, Horace (exp), Pythia (Pythia FSR), and Pythia (PHOTOS FSR). . . . . . . . . . . 133

5.5 Results of the FSR re-weighting and the change of $\sin ^{2} \theta_{\text {eff. }} \ldots \ldots 134$

5.6 Slope of the profile plots shown in Fig. $5.19 \ldots \ldots \ldots$. . . . . 137

5.7 Differences of the fitted $\sin ^{2} \theta_{\text {eff }}$ value in four different $p_{T}(Z)$ ranges for data and simulation . . . . . . . . . . . . . . . . 140

5.8 Correction to the $\sin ^{2} \theta_{\text {eff }}$ fit result in data and MC due to NLO (QCD)

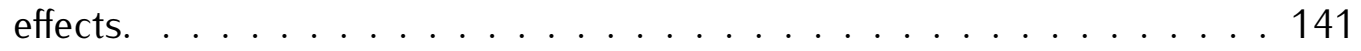

6.1 List of signal scenarios used in the case study . . . . . . . . . 151

6.2 Results of the hypothesis separation of the seven scenarios listed in Table 6.1 for $m_{X}=250 \mathrm{GeV}$. . . . . . . . . . . . . . . . . 164

6.3 Results of the hypothesis separation of the seven scenarios listed in Table 6.1 for $m_{X}=1 \mathrm{TeV}$.

6.4 Results of the fit for the free parameters of the spin-zero hypothesis with generated samples of SM Higgs-like $X$ corresponding to $0^{+}$. . 168 


\section{LIST OF TABLES}

6.5 Results of the fit for the free parameters of the spin-zero hypothesis with generated samples of a pseudo-scalar $X$ corresponding to $0^{-}$. . . 168

6.6 Results of the fit for the free parameters of the spin-one hypothesis with generated samples of an exotic pseudo-vector $X$ corresponding to $1^{+}$.

6.7 Results of the fit for the free parameters of the spin-one hypothesis with generated samples of an exotic vector $X$ corresponding to $1^{-}$. . . 169

6.8 Results of the fit for the free parameters of the spin-two hypothesis with positive parity with generated samples of an exotic $X$ corresponding to

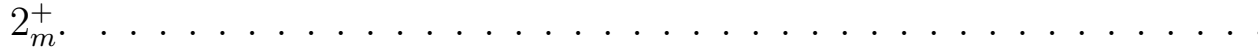

6.9 Results of the fit for the free parameters of the spin-two hypothesis with positive parity with generated samples of an exotic $X$ corresponding to

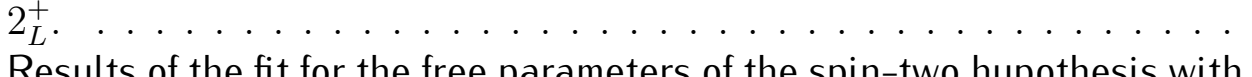

6.10 Results of the fit for the free parameters of the spin-two hypothesis with negative parity with generated samples of an exotic $X$ corresponding to $2^{-}$.

6.11 Results of the hypothesis separation $(\mathcal{S})$ of the seven scenarios for $m_{X}=250 \mathrm{GeV}$. with the $X \rightarrow Z Z \rightarrow 4 l$.

6.12 Results of the hypothesis separation $(\mathcal{S})$ of the seven scenarios for $m_{X}=500 \mathrm{GeV}$. with the $X \rightarrow Z Z \rightarrow 4 l$.

6.13 Results of the hypothesis separation $(\mathcal{S})$ of the seven scenarios for $m_{X}=1 \mathrm{TeV}$ with the $X \rightarrow Z Z \rightarrow 4 l$.

6.14 Results of the hypothesis separation of the $0^{+}$and $0^{-}$scenarios for $m_{X}=140 \mathrm{GeV}$.

6.15 Number of $Z Z$ background events from $m_{4 l}=[100-600] \mathrm{GeV}$ for two methods of estimation . . . . . . . . . . . . . . . . 183

6.16 Number of expected events and relative uncertainties for reducible for reducible background contributions $t \bar{t}, Z b \bar{b} / c \bar{c}$ and $Z$ +jets . . . . . . 184

6.17 Summary of the magnitude of systematic uncertainties in percent. . . . 185 


\section{List of Figures}

1.1 Representation of the particles in the Standard Model . . . . . . . . . 3

1.2 Indirect constraints on the Higgs mass from electroweak precision measurements and direct constraints from LEP and the Tevatron, previous to this work. . . . . . . . . . . . . . . 6

1.3 The composition of the cosmos ................ 8

1.4 Landscape of BSM physics models as envisioned by H. Murayama [10] 10

2.1 Euler angles. . . . . . . . . . . . . . . . . 16

2.2 The helicity states for a spin-1/2 particle are illustrated. . . . . . . . 19

2.3 Examples are given for the electromagnetic and weak neutral interactions. 23

2.4 Weak charged current interactions . . . . . . . . . . . . 24

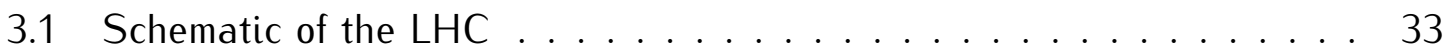

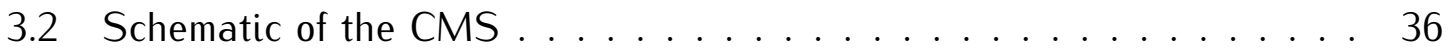

3.3 Functionality of CMS subsystems . . . . . . . . . . . 37

3.4 Drawing of the CMS inner tracking system . . . . . . . . . . . 39

3.5 CMS muon system ........................... 43

3.6 Distribution of cosmic ray muons in data and simulation . . . . . . . . 46

3.7 Visualization of track residuals . . . . . . . . . . . . . . . 50

3.8 Normalized residuals for various geometries . . . . . . . . . . . . . . 54

3.9 Track $\chi^{2} /$ ndf for various geometries . . . . . . . . . . . 55

3.10 Schematic of cosmic track splitting procedure. . . . . . . . . . . 56

3.11 Track parameter resolution using split cosmic tracks . . . . . . . . . . 57

3.12 Transverse impact parameter and $p_{T}$ resolution as a function of $p_{T} \quad$. . 58

3.13 Comparison of module positions between combined and design geome-

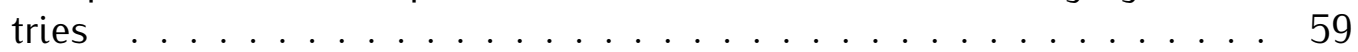

3.14 Geometrical deformation and track $\chi^{2}$ performance in weak mode align-

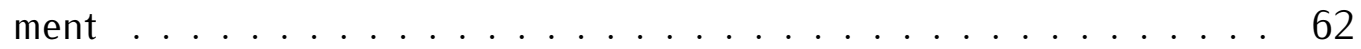

4.1 Parton-parton luminosities at the LHC . . . . . . . . . . . . 66 


\section{LIST OF FIGURES}

4.2 Production mechanisms for particle $X$ at the LHC . . . . . . . . 66

4.3 Decay channels for particle $X$ at the LHC . . . . . . . . . . . . . 67

4.4 Angular distribution for $2 \rightarrow 4$ process. . . . . . . . . . 83

4.5 Virtual $Z$ invariant mass, $m_{*}$, for a scalar and pseudoscalar spin-zero

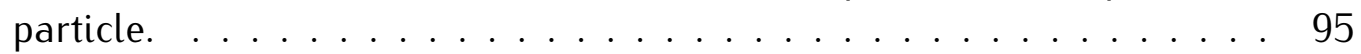

5.1 Drell-Yan process . . . . . . . . . . . . . . . . . . . 98

5.2 The production angle $\theta^{*}$ defined in the $X$ rest frame. . . . . . . . 103

5.3 Forward-backward asymmetry $A_{F B}$ as a function of mass . . . . . . . 105

5.4 Analytical parameterization of the Parton Distribution Functions using the CTEQ6L [54] numerical calculation. . . . . . . . . . . . . . . . 107

5.5 Distribution of the effective cross-section factor $F_{q \bar{q}}\left(m^{2}, Y=0\right) . \quad \ldots 111$

5.6 Distribution of the dilution factor $D_{q \bar{q}}\left(m=m_{Z}, Y\right)$. . . . . . . . 112

5.7 Distributions of $Y, m, \cos \theta^{*}$ for the analytical model and PYTHIA simulation for individual quark flavor contributions. . . . . . . . . . 113

5.8 Comparison of numerical PDF computation CTEQ6L versus the analytical description. . . . . . . . . . . . . . . 115

$5.9 Q$-dependence of the PDF fit parameters. . . . . . . . . . . 115

5.10 Distribution of the angle between the two muon tracks in data and signal MC . . . . . . . . . . . . . . . . . . . 118

5.11 Distribution of MC (POWHEG) events after progressive kinematic cuts 119

5.12 Accepted $\cos \theta^{*}$ range as a function of $Y$ for the kinematic selection in analysis . . . . . . . . . . . . . . . . . . . 122

5.13 Total efficiency of trigger, reconstruction, and acceptance as a function of muon $\eta$, dilepton $Y, \cos \theta^{*}$, and $m \ldots \ldots$. . . . . . . 123

5.14 The efficiency function $\mathcal{G}_{2}$ fitted from using simulation . . . . . . . . . . 124

5.15 Distribution of $Y, m, \cos \theta^{*}$ for the analytical model and PYTHIA simulation before and after detector acceptance, efficiency, and resolution and FSR effects. . . . . . . . . . . . . . . . . . . 126

5.16 Distributions of the backgrounds to the Drell-Yan process . . . . . . 127

5.17 Effect on $\sin ^{2} \theta_{W}$ of PDF variations for various PDF models. . . . . . . 131

5.18 FSR tail in the range $60<m<90 \mathrm{GeV}$ in the process $q \bar{q} \rightarrow \gamma^{*} / Z \rightarrow$ $\mu^{-} \mu^{+}$generated with four different FSR models . . . . . . . . 133

5.19 Profile of the di-lepton invariant mass distribution as a function of $\cos \theta^{*} \ldots \ldots \ldots \ldots \ldots \ldots 137$

5.20 Invariant mass of the di-lepton pair in simulation for the design and Realistic geometries . . . . . . . . . . . . . . . 138

$5.21 p_{T}$ distribution of the di-muon system in data and simulation and the corresponding ratio ..................... 140

5.22 Distribution of $Y, \cos \theta^{*}, m$ in the analysis of $q \bar{q} \rightarrow \gamma^{*} / Z \rightarrow \mu^{-} \mu^{+}$ events from POWHEG-based CMS detector simulation. . . . . . . . 147 


\section{LIST OF FIGURES}

5.23 Distribution of $Y, \cos \theta^{*}, m$ in the analysis of $q \bar{q} \rightarrow \gamma^{*} / Z \rightarrow \mu^{-} \mu^{+}$ events from LHC data.

6.1 Distribution of the angular observables for the various signal scenarios in the case study and the background . . . . . . . . . . 154

6.2 Detector acceptance effects for $\cos \theta^{*}$ and $\Phi_{1} \ldots \ldots \ldots \ldots \ldots$

6.3 Separations of signal hypothesis for $0^{+}$and $0^{-}$(left) and $0^{+}$and $2_{m}^{+}$

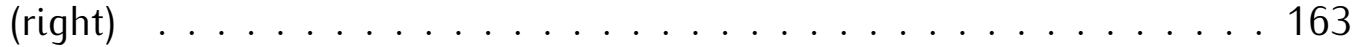

6.4 Fit for the parameters in Table 6.4 for a $0^{+}$signal hypothesis for 1000 toy experiments. . . . . . . . . . . . . . . . 170

6.5 Projection off-shell $Z$ mass for three samples at $140 \mathrm{GeV}$ : signal $0^{+}$, signal $0^{-}$, and background. . . . . . . . . . . . . . . 174

6.6 Production cross-section for SM Higgs at $7 \mathrm{TeV} \ldots \ldots \ldots \ldots$

6.7 Branching ratios for SM Higgs at $7 \mathrm{TeV} \ldots \ldots \ldots \ldots \ldots$

6.8 Leading-order invariant mass distribution for the $Z Z$ background contribution (points) and the fitted PDF (lines). . . . . . . . 188

6.9 NLO contributions for the $Z Z$ backgrounds for $q q \rightarrow Z Z$ (left) and $g g \rightarrow Z Z$ (right) . . . . . . . . . . . . . . . 189

6.10 Fits of simulated Higgs signal events for three different mass points . 190

6.11 Breit-Wigner-like and Crystal Ball function parameters as a function of mass for the signal mass parameterization . . . . . . . . . . 191

6.12 Signal efficiencies for the $4 e, 4 \mu$ and $2 e 2 \mu$ channels. . . . . . . . 192

6.13 All input shapes are shown for signal $(300 \mathrm{GeV})$ and background including data points ................... . 193

6.14 The mean expected and the observed upper limits at 95\% C.L. on $\sigma(p p \rightarrow$ $\mathrm{H}+\mathrm{X}) \times \mathcal{B}(\mathrm{ZZ} \rightarrow 4 \ell) \quad \ldots \ldots \ldots \ldots \ldots \ldots \ldots$

6.15 Combined CMS Higgs Limits with 1.1 to $1.7 \mathrm{fb}^{-1}$. . . . . . . . . 197 


\section{Chapter 1}

\section{Introduction}

"But one thing hasn't changed: you must help your humans, not hinder them. You must help them and guide them and encourage them towards wisdom. That's what daemons are for."

-Serafina Pekkala to Pan and Kirjava

"The Amber Spyglass", Phillip Pullman

\subsection{The Standard Model and the LHC era}

The Large Hadron Collider (LHC) [1] is a proton-proton collider at CERN (European Center for Nuclear Research) near Geneva, Switzerland. It collides protons at energies higher than the world's previously highest energy collider, the Tevatron [2]. At this new energy, particle physicists will finally be able to investigate many of the mysteries which currently plague the field.

Perhaps a concise, though simplified, way to understand the main principles of 


\section{CHAPTER 1. INTRODUCTION}

particle physics is to ask two elementary questions:

- What is the stuff we are all made of?

- How does that stuff interact?

Since the time of the ancient Greeks, we have been trying to understand the most basic building blocks of nature, the most fundamental properties of matter. While the level of sophistication over the many years has improved, many of the basic questions have not.

The Standard Model (SM) of particle physics [3] is the current framework which particle physicists use to describe the universe. Since its conception in the 1970s, it has stood the test of time and many precision tests. The SM consists of two types of particles: fermions and bosons which are represented in Fig. 1.1. Fermions are the matter particles in the SM (the stuff) and have intrinsic half-integer spin which obey Fermi-Dirac statistics and cannot occupy the same quantum state. Bosons are the particles in the SM which mediate the interactions between the fermions (how the stuff interacts) and have intrinsic integer spin which obey Bose-Einstein statistics and can occupy the same quantum state. The spins of these fundamental particles is a particularly relevant property which will be relevant throughout this work and can be thought of as the intrinsic angular momentum of the particle.

Further the matter particles are divided into two types: leptons and quarks; both the leptons and quarks are further divided into three families or flavors. The quarks 


\section{CHAPTER 1. INTRODUCTION}

consist of up-type quarks with charge $q=2 / 3$ : up, charm, and top and down-type quarks with charge $q=-1 / 3$ : down, strange, bottom. The three families of leptons are the electron and electon neutrino, the muon and muon neutrino, and the tau and tau neutrino. The electron, muon and tau have charge $q=-1$ and the neutrinos, as the name dictates, have charge $q=0$. As the family goes from left to right in Fig. 1.1, the masses of the quarks and leptons increase.

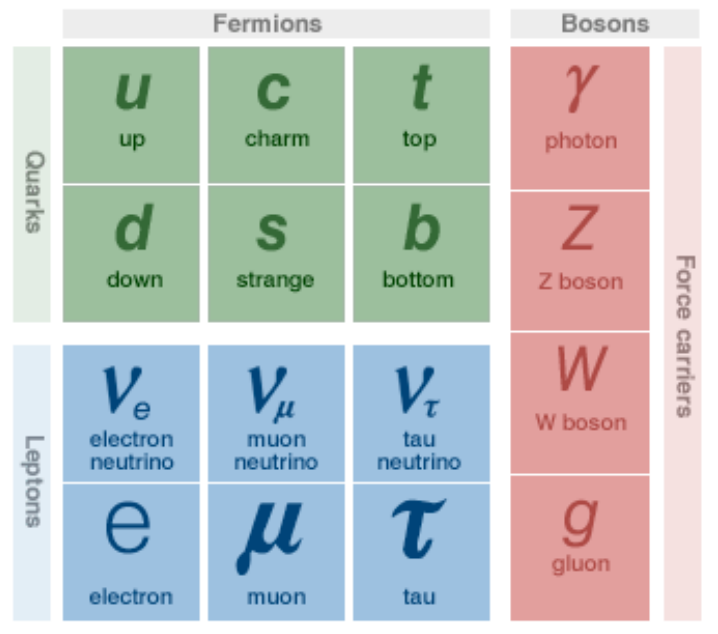

Figure 1.1: Representation of the particles in the Standard Model

In addition to the matter particles in the SM, there are also their mirror particles, anti-matter. For each matter particle, there is an exact replica particle with the same mass, but opposite charge. For example, we have the anti-up quark with charge $q=-2 / 3$ denoted as $\bar{u}$ and the anti-muon with charge $q=+1$ denoted as $\mu^{+}$.

The bosons are the particle carriers which mediate the fundamental forces of the SM. The fundamental forces described by SM are the electromagnetic force mediated 


\section{CHAPTER 1. INTRODUCTION}

by the photon, $\gamma$; the weak force mediated by the $W^{ \pm}$and $Z^{0}$ bosons; and the strong force mediated by the gluons, $g$. There is one force absent from the model, gravity, and its absence will be discussed further in Sec. 1.2.

With these building blocks of matter and the forces governing them, we can begin to construct the world around us. The quarks, held together by strong interactions via gluons, are combined to make protons and neutrons. Protons (neutrons) consist primarily of two (one) up quarks and one (two) down quarks and the gluons holding it together as well as a sea of quark-antiquark pairs. The electrons are bound to the nuclei consisting of protons and neutrons via the electromagnetic force to form the basic atom. From the atoms, we have our familiar periodic table of elements, and these elements make up much of the luminous matter in the universe.

\section{The Higgs boson and the origin of mass}

Though the initial description of the SM seems rather haphazard, there is an underlying mathematical construction which naturally expresses the model. The SM, much like the rest of physics, is governed by symmetries and each fundamental force and its interaction with matter is described by a symmetry group. This will be described in greater detail in Sec. 2.3.

For now, it is simply posited that the underlying symmetry of the SM requires that all the particles are massless. However, we know that weak bosons, $W^{ \pm}$and $Z^{0}$, are massive as well as all the fermions in the SM. The reason for this is that 


\section{CHAPTER 1. INTRODUCTION}

the electroweak symmetry, the symmetry of the electromagnetic and weak forces, is broken giving the weak bosons mass. A solution for electroweak symmetry breaking is the Higgs mechanism whereby the electroweak symmetry is spontaneously broken and a new spin zero particle, the Higgs boson, is manifest. It is the interaction of the Higgs field with the fermions which gives them mass.

However, the Higgs boson remains elusive. Direct searches for it have so far proven fruitless. Figure 1.2 [4] shows indirect constraints on the Higgs boson mass from precision electroweak measurements and direct constraints from the LEP [5] and Tevatron [6] experiments; the white region shows where the Higgs mass has not yet been ruled out experimentally. While the search for the Higgs continues, the discovery of the nature of electroweak symmetry breaking in the SM is one of the major goals of the LHC.

\subsection{Beyond the Standard Model}

Besides the search for the nature of electroweak symmetry breaking, there are other mysteries which cannot be explained by the SM. The principal ones are outlined below though there are several other mysteries which are not discussed. They are divided into two categories: questions about the nature of the SM and evidence of cosmological inconsistencies. 


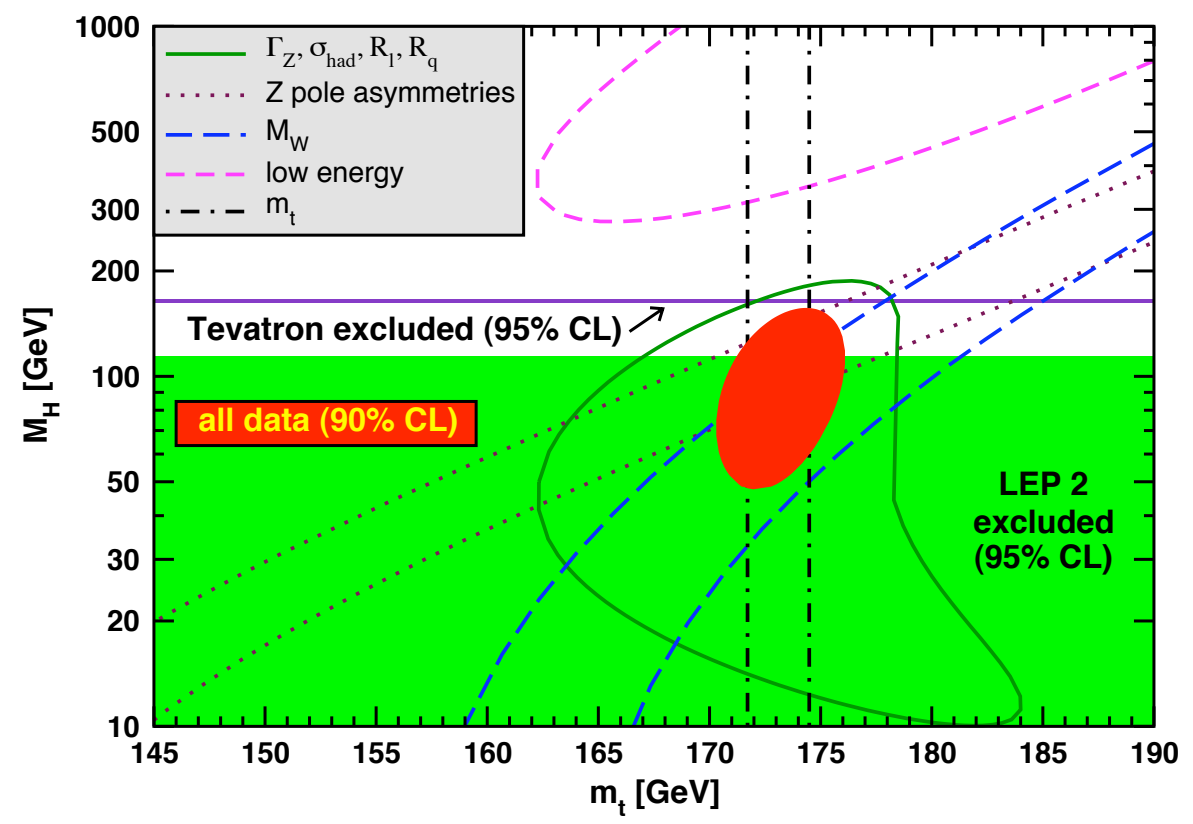

Figure 1.2: Indirect constraints on the Higgs mass from electroweak precision measurements and direct constraints from LEP and the Tevatron, previous to this work.

\section{Beyond the SM of particle physics}

One of the main issues with the SM is so-called the hierarchy problem. The idea is distilled simply to one question: why are the other forces so much stronger than the gravitational force? In fact, it is some 32 orders of magnitude $\left(10^{32}\right)$ weaker than the weak force at the order of the Plank scale, the energy scale at which quantum and gravitational effects are equal. Because of this vast difference between the forces, the model requires some "fine-tuning", where selection of the parameters of the SM are 


\section{CHAPTER 1. INTRODUCTION}

very precise at the order of $\sim 10^{-32}$. This fine-tuning is somehow undesirable within the model as though it is fabricated without reasonable theoretical motivation.

Further, other questions remain such as why there are three families of fermions. There is no particular reason why there should be only three families and so perhaps, there are even more families at higher energies. In addition, there is no pattern for why the fermion masses are what they are; this is known as the flavor problem. The masses of the fermions are simply parameters of the SM with no theoretical motivation.

As mentioned previously, gravity is not accounted for in the SM, which means that there is some other, more complete, description of the universe. If the magnitude of the four forces are calculated at the Planck energy scale, one finds that they are all almost equal; this is described as grand unification. The way that these forces are unified and the mathematical model which describes it is unknown and evidence that our current SM is not the whole picture.

\section{Cosmological mysteries}

The intersection between particle physics and astrophysics provides further evidence of inconsistencies with the SM. Cosmological evidence points to the existence of non-luminous dark matter. Originally discovered by the observation that the velocity of galaxies are not in agreement with their luminous mass [7], it appears the much of the matter in the universe is cold (moving at non-relativistic speeds) and dark (non-luminous) which does not interact strongly with the luminous matter. Further, 


\section{CHAPTER 1. INTRODUCTION}

it appears as though the rate of expansion of the universe is greater than expected pointing to some hypothetical energy which permeates the universe - dark energy [8]. In accounting for the presence of dark matter and dark energy, $95 \%$ of the composition of the cosmos consists of that which is not described in the SM. This is illustrated in

Fig. 1.3

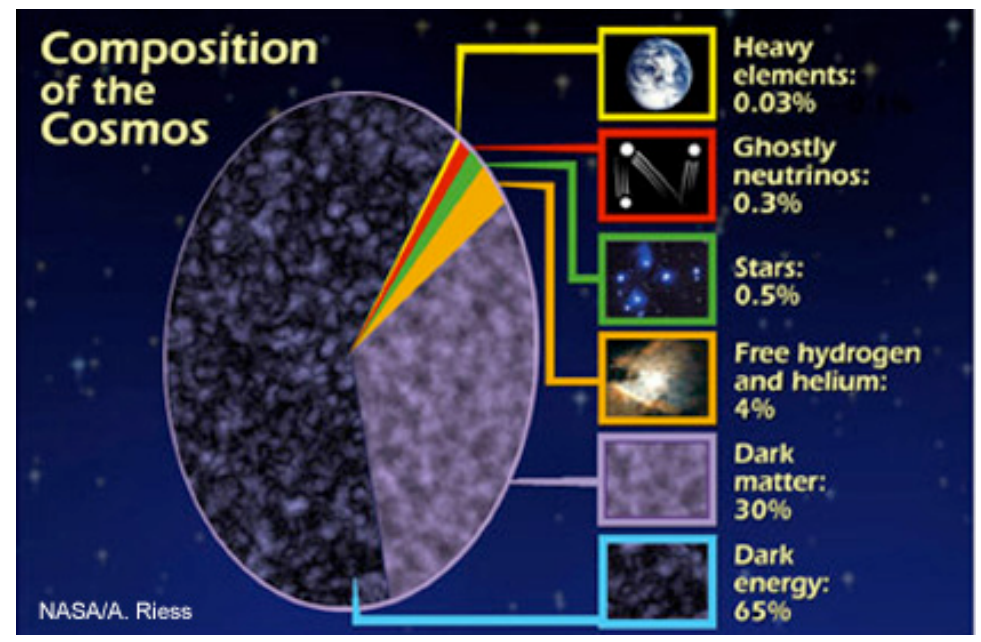

Figure 1.3: The composition of the cosmos

Further, another cosmological mystery is the asymmetry of matter and antimatter in the universe. The universe is made predominantly of matter, and the conditions for this asymmetry are often referred to as the Sakharov criteria [9]. The SM has a mechanism whereby this can occur; however, this mechanism is not strong enough to account for the amount of matter in the universe and the reason remains a mystery. 


\section{CHAPTER 1. INTRODUCTION}

\section{BSM models}

With such mysteries permeating the field of particle physics, there are many ideas, beyond the SM (BSM), which can account for these types of inconsistencies. Two such very popular models, supersymmetry and warped extra-dimensions, are briefly described though the entire landscape of beyond the SM physics is vast. Supersymmetry is an extension of the SM where a new symmetry is introduced which results in partner particles for all the SM particles. For each fermion, there is a corresponding supersymmetric boson, and for each boson, there is a corresponding supersymmetric fermion. In such types of supersymmetric models, a solution is given for the hierarchy problem as the new supersymmetric particles conspire to cancel the fine-tuning problem and many of this class of models give hypothetical dark matter particles. In warped extra-dimensional models, a very small and warped extra (fifth) dimension is hypothesized. Such classes of models yield a tower of new particle states which, among other things, account for hierarchy and flavor problems and provide dark matter candidates.

However, these two class of models only scratch the surface of possible beyond the SM phenomenology; the landscape of possibilities is vast and unexplored. This is illustrated in Fig. 1.4. Here, it is not only illustrated all the possible hypothesized models, but also it demonstrates our ignorance that the solution to beyond the SM physics has not even yet been hypothesized. 


\section{CHAPTER 1. INTRODUCTION}

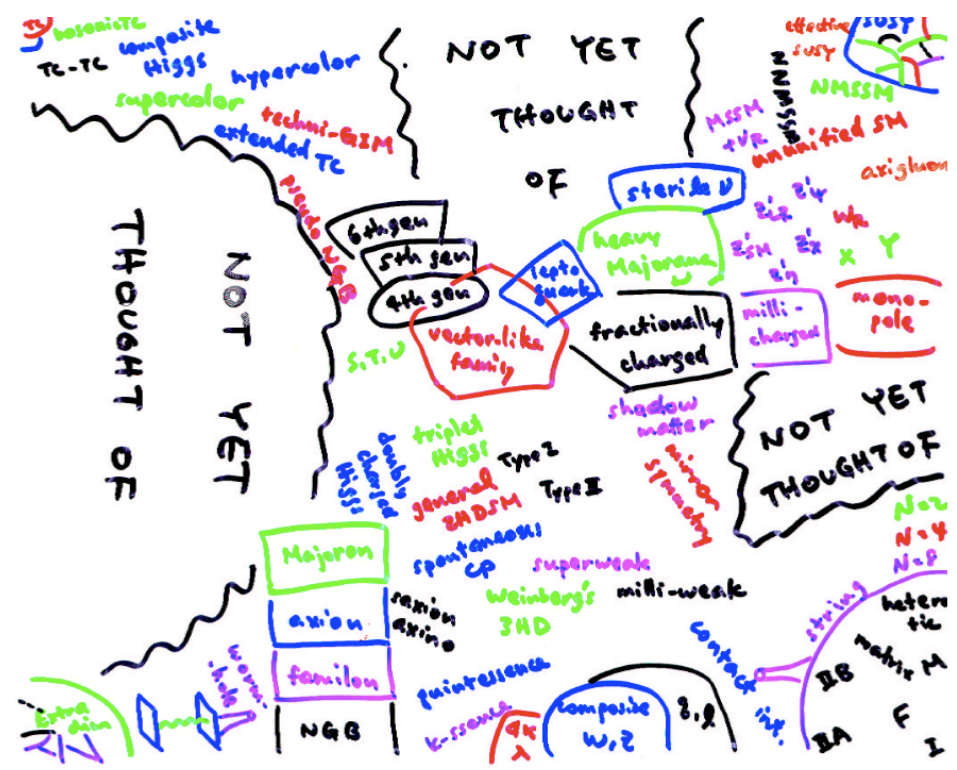

Figure 1.4: Landscape of BSM physics models as envisioned by H. Murayama [10]

\subsection{Angles and Daemons}

A brief description of the current status of particle physics has been laid out. The SM has been successful in describing much of what we know, but there are also many things which are not understood and remain a mystery. At the LHC, we hope to find evidence of the nature of electroweak symmetry breaking and beyond the SM physics. However, to find evidence of new physics is not enough, it must be understood. Many different BSM models predict new particles, but the nature of these particles, its spin, parity and interactions with the SM particles, must be determined so that the nature of any new physics is understood.

Described in the following chapters, methods and techniques are given which analyze the kinematics of the decay of such new particles. By understanding the kinematic 


\section{CHAPTER 1. INTRODUCTION}

and angular distributions of the decaying particles, the properties of the new resonance can be determined. For example, if a new particle is found to decay to two $Z^{0}$ bosons, is it the spin-zero Higgs boson, a spin-one heavier cousin of the $Z^{0}$ boson, or perhaps even a spin-two graviton? The angular distributions can give us a clue. It also serves as a reminder that if and when a new particle is discovered, this only begins the search for new physics.

The title of this thesis, Angles and Daemons ${ }^{1}$, signifies the idea of understanding new physics via its kinematics and angular distributions and the challenge and pitfalls in understanding any new physics we may encounter. Alternatively, daemons represents a bit of a play on words as Wikipedia ${ }^{2}$ describes daemons as "characters in Greek mythology, some of whom handled tasks that the gods could not be bothered with".

\footnotetext{
${ }^{1} \mathrm{~A}$ pun on the popular 2009 film "Angels and Demons" featuring scenes of the LHC, based on the 2001 novel by Dan Brown of the same name.

${ }^{2}$ http://en.wikipedia.org/wiki/Daemon_(computer_software)
} 


\section{Chapter 2}

\section{Physics foundations}

"Curiously enough, the only thing that went through the mind of the bowl of petunias as it fell was,

'Oh no, not again'. Many people have speculated that if we knew exactly why the bowl of petunias had thought that we would know a lot more about the nature of the universe than we do now."

- Douglas Adams, The Hitchhiker's Guide to the Galaxy

In this chapter, selected physics topics are introduced to better frame discussion in later chapters. A fully pedagogical approach is not taken, but rather a brief description with ample references is provided. The concepts of angular momentum and symmetries are developed in order to better understand particle kinematics. These ideas will be applied to the discussion of the Standard Model and particle interactions and dynamics at high energies. 


\section{CHAPTER 2. PHYSICS FOUNDATIONS}

\subsection{Angular Momentum}

In 'Modern Quantum Mechnics' by J. J. Sakurai [11], the author states that "the importance of angular momentum in modern physics can hardly be overemphasized". We will see that it is an integral part of many of the topics that will be discussed. The concepts in this section come mostly from Refs. [11-13].

\section{Angular momentum}

Recall from classical mechanics that rotations fail to commute, or in other words, the order in which rotations are applied matter. As an example, consider that a rotation of an object about its $x$-axis followed by a rotation about its $z$-axis does not necessarily end up in the same final state as the case as if it is rotated about its $z$-axis then the $x$-axis. This is what makes rotations unique from translations in three-dimensions, which do commute. Rotations of a given vector are generated by a $3 \times 3$ matrix, $R$, and by carefully considering infinitesimal rotations of $\epsilon$ around various axes, we find the commutation relation:

$$
R_{x}(\epsilon) R_{y}(\epsilon)-R_{y}(\epsilon) R_{x}(\epsilon)=R_{z}(\epsilon)
$$

From classical mechanics, we know that angular momentum is the generator of rotations; in other words, the angular momentum remains invariant under rotations. We now define the angular momentum operator $J_{k}$ as the generator of infinitesimal rotations about the axis $k$. In the quantum mechanical case, angular momentum is 


\section{CHAPTER 2. PHYSICS FOUNDATIONS}

quantized into units ${ }^{1}$ of $\hbar / 2$. A finite rotation of the quantum states $|\alpha\rangle$ is defined by the operator $\mathcal{D}(R)$ such that the rotated state is defined as $|\alpha\rangle_{R}=\mathcal{D}(R)|\alpha\rangle$. Then finite rotations can be described as a series of infinitesimal rotations,

$$
\mathcal{D}_{z}=1-\frac{i J_{z} \phi}{\hbar}-\frac{J_{z}^{2} \phi^{2}}{2 \hbar^{2}}+\ldots=\exp \left(\frac{-i J_{z} \phi}{\hbar}\right)
$$

If we assume that the rotation operator, $\mathcal{D}(R)$, obeys the same properties as the classic rotation $R$, we can determine the angular momentum commutation relations. Starting from Eq. 2.1, we find the fundamental commutation relations of angular momentum,

$$
\left[J_{i}, J_{j}\right]=J_{i} J_{j}-J_{j} J_{i}=i \hbar \varepsilon_{i j k} J_{k}
$$

This group of angular momentum operations in three dimensions above is said to be non-Abelian, meaning that they do not commute, and in the language of group theory, Eq. 2.3 forms a Lie algebra. The commutation relation algebra will give us the quantum mechanical angular momentum eigenvalues and eigenstates as well.

\section{Groups and $S U(2)$}

Group theory is a branch of mathematics which deals with the underlying treatment of symmetries. A group is defined by a set of axioms: closure, associativity, invertibility, and identity. As an example, the classical set of rotations characterized by a $3 \times 3$ orthogonal matrix $R$ forms a group. The group properties are satisfied:

\footnotetext{
${ }^{1}$ From now on, natural units are assumed where $\hbar=c=1$.
} 


\section{CHAPTER 2. PHYSICS FOUNDATIONS}

identity, $R \times \mathbb{I}=R$; closure, $R_{1} R_{2}=R_{3}$; associativity, $\left(R_{1} R_{2}\right) R_{3}=R_{1}\left(R_{2} R_{3}\right)$; and invertibility, $R R^{-1}=R^{-1} R=\mathbb{I}$. This group is known as $S O(3)$ where the $S$ stands for special, the $O$ for orthogonal since $R R_{T}=\mathbb{I}$ and the 3 for three dimensions.

In addition, it turns out we can also characterize arbitrary rotations in two dimensions - the lowest-dimension non-trivial representation of the rotation group. The generators can be written as $J_{i}=\sigma_{i} / 2$ where $\sigma_{i}$ are the Pauli matrices,

$$
\sigma_{1}=\left(\begin{array}{cc}
0 & 1 \\
1 & 0
\end{array}\right), \sigma_{2}=\left(\begin{array}{cc}
0 & -i \\
i & 0
\end{array}\right), \sigma_{3}=\left(\begin{array}{cc}
1 & 0 \\
0 & -1
\end{array}\right)
$$

The rotation operator in this representation is defined in the same way as in Eq. 2.2, written as $\mathcal{D}(\hat{n}, \phi)=\exp (-i \vec{\sigma} \cdot \hat{n} \phi / 2)$ and operates on states called two-component spinors, $\chi$, which can be generally written as the linear combination, $\chi=c_{+} \chi_{+}+c_{-} \chi_{-}$ of the eigenstates

$$
\chi_{+}=\left(\begin{array}{l}
1 \\
0
\end{array}\right), \chi_{-}=\left(\begin{array}{l}
0 \\
1
\end{array}\right)
$$

where $c_{+}$and $c_{-}$can be complex numbers. These spinors conventionally represent spin-1/2 $(J=1 / 2)$ particles with up or down spin projections $m=1 / 2$ or $m=-1 / 2$. This group is known as $S U(2)$ where the $S$ again stands for special, $U$ stands for unitary where now the rotation operator $\mathcal{D}$ is a unitary matrix, and 2 is the dimension of this representation. Besides its relationship to rotations, we will find that $S U(2)$ plays an important role in the structure of the SM. 


\section{CHAPTER 2. PHYSICS FOUNDATIONS}

\section{Wigner Functions}

In order to better describe rotations in particle interactions, we now consider rotations the the language of the Euler angles. In the classical case, the Euler rotations are defined as a rotation about the $z$-axis by an angle $\alpha$, a rotation about the $x$-axis by angle $\beta$, and then a rotation about the $z$-axis by an angle $\gamma$. This is presented in

Fig. 2.1. In the quantum mechanical case, the only change is that the second rotation will be taken about the $y$-axis. Then we can describe any arbitrary rotation as

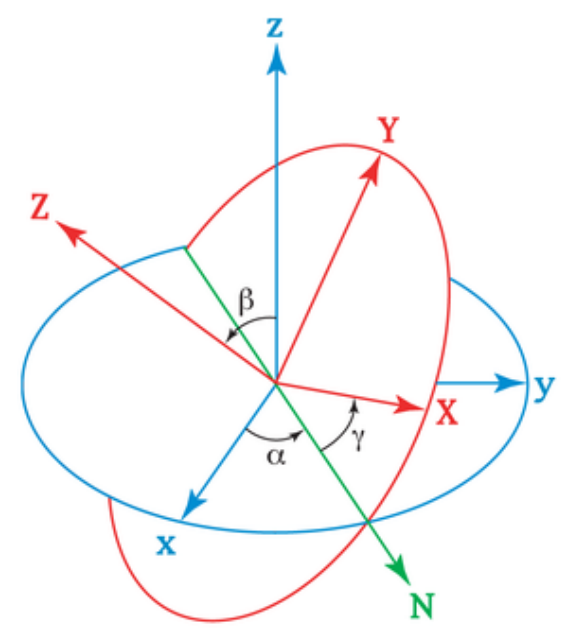

Figure 2.1: Euler angles

$\mathcal{D}(\alpha, \beta, \gamma)=\mathcal{D}_{z}(\alpha) \mathcal{D}_{y}(\beta) \mathcal{D}_{z}(\gamma)$. To write explicitly the rotation acting on a spin-1/2 particle, we have

$$
\mathcal{D}_{m^{\prime}, m}^{1 / 2}(\alpha, \beta, \gamma)=\left\langle J=\frac{1}{2}, m^{\prime}\left|e^{-i J_{z} \alpha} e^{-i J_{y} \beta} e^{-i J_{z} \gamma}\right| J=\frac{1}{2}, m\right\rangle
$$

Here, the initial state of a spin-1/2 system is $|J=1 / 2, m\rangle$ where $m$ can either be spin up or down as defined in Eq. 2.5. This initial state is then rotated to the final 


\section{CHAPTER 2. PHYSICS FOUNDATIONS}

state $\left\langle J=1 / 2, m^{\prime}\right|$ where $m^{\prime}$ can again be up or down but in the rotated frame. The function $\mathcal{D}_{m^{\prime}, m}^{J}$ is referred to as the Wigner functions. In the spin-1/2 case, we can work out the Wigner function by using the relation

$$
e^{i J_{k} \phi / 2}=\mathbf{1} \cos \frac{\phi}{2}-i \sigma_{k} \sin \frac{\phi}{2}
$$

Using this relation, we can write the Wigner functions as a function of the 'small' Wigner functions, $\mathcal{D}_{m^{\prime}, m}^{J}(\alpha, \beta, \gamma)=e^{-i m^{\prime} \alpha} d_{m^{\prime}, m}^{J}(\beta) e^{-i m \gamma}$. The individual elements for the small Wigner functions in the spin-1/2 case can then be computed as

$$
\begin{array}{r}
d_{1 / 2,1 / 2}^{1 / 2}(\beta)=d_{-1 / 2,-1 / 2}^{1 / 2}(\beta)=\cos (\beta / 2) \\
d_{1 / 2,-1 / 2}^{1 / 2}(\beta)=d_{1 / 2,-1 / 2}^{1 / 2}(\beta)=-\sin (\beta / 2)
\end{array}
$$

For the Wigner functions of arbitrary spin systems, we must discuss the angular momentum eigenvalues and eigenstates. Using only the angular momentum commutation relations in Eq. 2.3, we can determine that, for a spin- $J$ particle, there are $2 J+1$ allowed eigenstates [11] with values

$$
m=-J,-J+1, \cdots, J-1, J
$$

For a spin- $J$ system, we often refer to the Wigner functions $\mathcal{D}_{m^{\prime}, m}^{J}$ as the $(2 J+1)$ dimensional irreducible representation of the rotation operator. 


\section{CHAPTER 2. PHYSICS FOUNDATIONS}

\subsection{Helicity formalism}

In high energy particle physics at the scale of the LHC, often the collision energies are much greater than the mass of the colliding particles and can be thought of intersection of quantum mechanics and special relativity. In special relativity, we find that space and time are treated as equivalently in four dimensions. Further, we can equate energy and mass in Albert Einstein's famous equation $E^{2}=m^{2}+p^{2}$.

\section{Helicity states}

In the analogous way that a classical particle has the properties of energy and momentum, a quantum particle gains new properties for its quantum mechanical degrees of freedom, which we call quantum numbers. In the case of the intrinsic quantum mechanical angular momentum we have the spin quantum numbers. If we consider now a relativistic particle with non-zero spin in quantum mechanics, another "good" quantum number appears called helicity. The helicity operator is defined as

$$
H=\frac{\vec{\sigma} \cdot \vec{p}}{|\vec{p}|}
$$

where the helicity of a particle is defined as the projection of the spin along the direction of motion. For a spin-1/2 particle, the possible eigenvalues of the helicity operator are $\lambda=-1 / 2$ and $1 / 2$. Similar to the quantum eigenstates of angular momentum, the only allowed helicity states for a massive general spin- $j$ particle [13] 


\section{CHAPTER 2. PHYSICS FOUNDATIONS}

are

$$
\lambda=-J,-J+1, \ldots, J-1, J
$$

In the case of a massless particle, essentially when $E \gg m$ and $E^{2} \simeq p^{2}$, the formalism still holds but the allowed helicity states of a massless particle are

$$
\lambda= \pm J
$$

In the context of the Standard Model, we say that a spin-1/2 particle with its spin projection in the same direction as its momentum is said to be "right-handed" (RH) and when the spin projection is in the opposite direction of the momentum, it is said to be "left-handed" (LH). This is represented in Fig. 2.2. For massless neutrinos in the SM, all neutrinos are $\mathrm{LH}$ while all anti-neutrinos are $\mathrm{RH}^{2}$. The "handedness" of fermions in the SM is often referred to as chirality. For the case of massive fermions like quarks, electrons, muons and taus, they can be both $\mathrm{LH}$ and $\mathrm{RH}$.

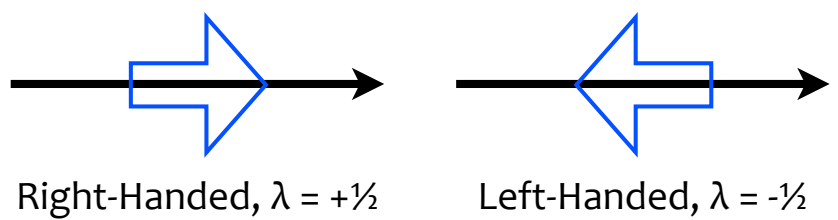

Figure 2.2: The helicity states for a spin-1/2 particle are illustrated.

\footnotetext{
${ }^{2}$ With the discovery of neutrino oscillations, neutrinos are discovered to have a small but finite mass and the $\mathrm{LH} / \mathrm{RH}$ nature of the neutrinos is not exact.
} 


\section{CHAPTER 2. PHYSICS FOUNDATIONS}

\section{$S$-matrix}

So far, we have discussed the angular momentum quantum states of a relativistic particle. However, to achieve our goal of describing the kinematics of particle interactions, we must discuss the general analysis of collisions. The $S$-matrix (or scattering matrix) formalism is the description of the evolution of a particle system. Our goal is not to fully describe the $S$-matrix theory ${ }^{3}$, but to incorporate the idea of helicity states into the formalism.

Consider a particle reaction, $a+b \rightarrow c+d$. which can be designated with momenta and helicity states

$$
\left\langle\vec{p}_{c}, \vec{p}_{d} ; \lambda_{c} \lambda_{d}|S| \vec{p}_{a}, \vec{p}_{b} ; \lambda_{a}, \lambda_{b}\right\rangle
$$

where $S$ is the matrix element describing the interaction properties and $P_{\mu}$ and $P_{\mu}^{\prime}$ are the total initial and final four-momenta, respectively. Reference systems can be defined arbitrarily for the initial and final states. The reference system for the initial state is defined by the azimuthal and polar angles $\phi_{0}$ and $\theta_{0}$ while the final state is defined by $\phi$ and $\theta$. In the center-of-mass frame of the reaction, $P_{\mu}=P_{\mu}^{\prime}=0$, we can then choose our reference system along the $z$-direction of the initial system such that $\theta_{0}=0$ and $\phi_{0}$ is arbitrary. Now, Eq. 2.13 becomes

$$
\left\langle\vec{p}_{c}, \vec{p}_{d} ; \lambda_{c} \lambda_{d}|S| \vec{p}_{a}, \vec{p}_{b} ; \lambda_{a}, \lambda_{b}\right\rangle \sim \delta^{4}\left(P_{\mu}-P_{\mu}^{\prime}\right)\left\langle\phi, \theta ; \lambda_{c} \lambda_{d}|S(E)| 0,0 ; \lambda_{a}, \lambda_{b}\right\rangle
$$

\footnotetext{
${ }^{3}$ see, for example, [14], for a more complete description
} 


\section{CHAPTER 2. PHYSICS FOUNDATIONS}

And we reduce Eq. 2.14 including the Wigner functions as

$$
\begin{aligned}
\left\langle\phi, \theta ; \lambda_{c} \lambda_{d}|S(E)| 0,0 ; \lambda_{a}, \lambda_{b}\right\rangle & =\frac{1}{4 \pi} \sum_{J}(2 J+1)\left\langle\lambda_{c} \lambda_{d}\left|S^{J}(E)\right| \lambda_{a} \lambda_{b}\right\rangle \mathcal{D}_{\lambda, \mu}^{J}(\phi, \theta, \phi) \\
& =\frac{1}{4 \pi} \sum_{J}(2 J+1)\left\langle\lambda_{c} \lambda_{d}\left|S^{J}(E)\right| \lambda_{a} \lambda_{b}\right\rangle e^{-i(\lambda-\mu) \phi} d_{\lambda, \mu}^{J}(\theta) \\
& =\frac{1}{4 \pi} \sum_{J}(2 J+1) A_{\lambda, \mu} e^{-i(\lambda-\mu) \phi} d_{\lambda, \mu}^{J}(\theta)
\end{aligned}
$$

where $J$ is the total angular momentum of the system, $\lambda=\lambda_{a}-\lambda_{b}$, and $\mu=\lambda_{c}-\lambda_{d}$. Eq. 2.15 is the main result of this section and gives the foundation for exploring dynamics of any $2 \rightarrow 2$ particle reaction. In particular, we redefine

$$
A_{\lambda, \mu}=\left\langle\lambda_{c} \lambda_{d}\left|S^{J}(E)\right| \lambda_{a} \lambda_{b}\right\rangle
$$

and call $A_{\lambda, \mu}$ the helicity amplitude. It is the amplitude for a given process to proceed in a given angular momentum configuration at a given energy, $\left\langle\lambda_{c} \lambda_{d}\left|S^{J}(E)\right| \lambda_{a} \lambda_{b}\right\rangle$. From the $S$-matrix element to get to the usual differential cross-section which gives the phase-space for the kinematics of a given process, we use the relationship [13]

$$
d \sigma \propto\left|\left\langle\phi, \theta ; \lambda_{c} \lambda_{d}|S(E)| 0,0 ; \lambda_{a}, \lambda_{b}\right\rangle\right|^{2} d \Omega
$$

where $d \Omega=d(\cos \theta) d \phi$ is the differential over the solid angle.

\subsection{The Standard Model, redux}

One of the most beautiful results from physics is the relationship between conserved quantities and symmetries of a system. Noether's theorem states that every 


\section{CHAPTER 2. PHYSICS FOUNDATIONS}

conserved quantity corresponds to an invariance of the equations of motion under a given symmetry operation [15]. Examples from classical physics are:

- translational invariance $\Leftrightarrow$ conservation of momentum

- rotational invariance $\Leftrightarrow$ conservation of angular momentum

- invariance under spatial reflection $\Leftrightarrow$ parity conservation

- time invariance $\Leftrightarrow$ conservation of energy

These symmetries are known as external symmetries and deal with four-dimensional space-time. In particular, the parity symmetry will be revisited. Parity can be thought of as the discrete transformation from a left-handed system to a right-handed system where a field is considered parity-even if it is constant under the parity operator and parity-odd if it is changes sign under the parity operator.

In contrast, we have internal symmetries that deal with the internal symmetries of particle wave functions, additional degrees of freedom. In particular, the idea of local (gauge) symmetries is central to modern quantum field theory. This gauge symmetry is related to the phase of the quantum wave function of a particle. While we do not go into great detail about quantum field theory, it is often a "natural" requirement for gauge symmetry of free particle fields. This requirement of gauge symmetry requires a new vector field (boson) which gives our model of interactions with free particle fields. This new vector field, by this construction, should be massless. 


\section{CHAPTER 2. PHYSICS FOUNDATIONS}

\section{Electroweak unification}

In Section 1.1, we introduce the structure of the Standard Model. The description includes the matter particles of half-integer spin called fermions which consists of quarks and leptons and the force carrier particles of integer spin called bosons. The interactions of elementary particles are mediated by the exchange of these force particles. In Fig. 2.3, we see the interactions of a fermion with a boson for both the electromagnetic and weak force. The illustrations presented in Fig. 2.3 are known as
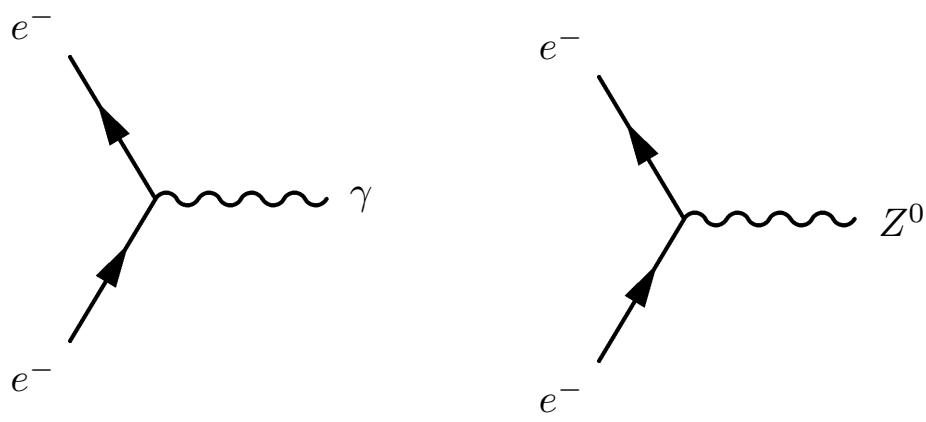

Figure 2.3: Examples are given for the electromagnetic and weak neutral interactions.

Feynman diagrams which graphically represent a particle interaction process and also are quantitatively associated with the particular transition amplitude of the process. In Fig. 2.3 time runs from bottom-to-top.

By construction, the structure of the Standard Model is governed by the set of symmetries, $S U(3)_{C} \times S U(2)_{L} \times U(1)_{Y}$. The $S U(3)_{C}$ symmetry of quantum chromodynamics is left without discussion and here we focus on the $S U(2)_{L} \times U(1)_{Y}$ electroweak symmetry. Fermions in the SM are described by Dirac spinors for both particles and 


\section{CHAPTER 2. PHYSICS FOUNDATIONS}

antiparticles denoted as $u$ and $v$, respectively [12]. For relativistic fermions where $E \gg m$, we can have left-handed and right-handed Dirac spinors which are eigenstates of the Dirac matrix ${ }^{4}$ operator $\gamma^{5}$,

$$
\begin{gathered}
\gamma^{5} u_{R}=u_{R}, \gamma^{5} u_{L}=-u_{L} \\
\gamma^{5} v_{R}=-v_{R}, \gamma^{5} v_{L}=v_{L}
\end{gathered}
$$

where $u=u_{R}+u_{L}$ are the spinors of a given particle and $v=v_{R}+v_{L}$ are the spinors of a given anti-particle. It follows then that for a purely left-handed interaction is characterized by

$$
\frac{1-\gamma^{5}}{2} u_{R}=0, \frac{1-\gamma^{5}}{2} u_{L}=u_{L}
$$

It has been observed that the nature of weak charged current interactions has the same structure which couples only to left-handed currents as the interaction in Fig. 2.4 has the form $\sim \gamma_{\mu}\left(1-\gamma^{5}\right) / 2$. Observing the structure of the weak interactions, we
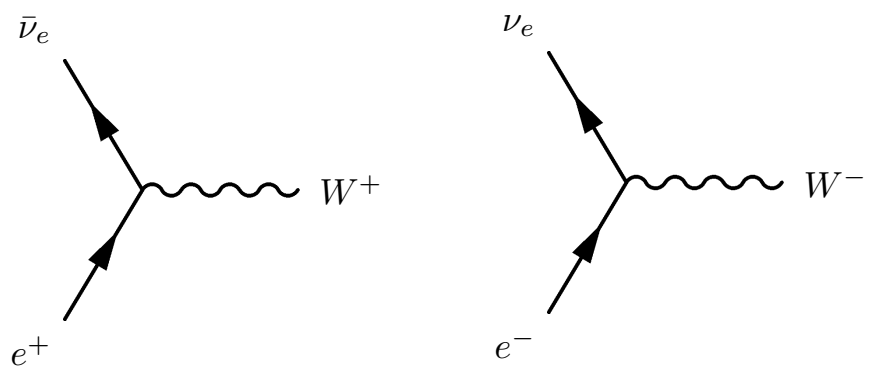

Figure 2.4: Weak charged current interactions

notice that it is similar to the $S U(2)$ structure described in Section 2.1 where instead

${ }^{4}$ For further discussion, see [16] 


\section{CHAPTER 2. PHYSICS FOUNDATIONS}

of the doublet consisting of up and down spin projections, we have the left-handed doublet and a right-handed singlet

$$
\chi_{L}=\left(\begin{array}{c}
\nu \\
e^{-}
\end{array}\right)_{L}, \quad \chi_{R}=\left(e^{-}\right)_{R}
$$

This structure comes from the property that neutrinos are exclusively left-handed. We can now write the weak charged current interaction in the shorthand

$$
J_{\mu} \equiv \bar{\nu} \gamma_{\mu} \frac{1}{2}\left(1-\gamma^{5}\right) e=\bar{\nu}_{L} \gamma_{\mu} e_{L}
$$

In this $S U(2)_{L}$ symmetry, we make a direct analogy to the angular momentum $S U(2)$ group in quantum mechanics. This new conserved quantity is called weak isospin, and we can generate the $S U(2)_{L}$ with the weak isospin generators $\vec{\tau}$ which follow the same commutation relation,

$$
\left[\tau_{i}, \tau_{j}\right]=\varepsilon_{i j k} \tau_{k} .
$$

The third generator corresponding to the weak neutral current interaction, we then anticipate should also have the purely left-handed structure of $J_{3} \equiv \bar{\nu}_{L} \gamma_{\mu} \nu_{L}+$ $\bar{e}_{L} \gamma_{\mu} e_{L}$. However, this is not what is observed in nature as the weak neutral current involves both right-handed and left-handed currents. To save our $S U(2)$ weak isospin symmetry, we observe that the electromagnetic force contains both $\mathrm{LH}$ and $\mathrm{RH}$ currents via the charge operator $Q$

$$
J_{\mu}^{e m} \equiv \bar{e}_{R} \gamma_{\mu} Q e_{R}+\bar{e}_{L} \gamma_{\mu} Q e_{L}
$$




\section{CHAPTER 2. PHYSICS FOUNDATIONS}

$Q$ is the generator of the $U(1)_{e m}$ symmetry group of electromagnetic interactions. To preserve the weak isospin symmetry, we create a new hypercharge operator, $Y$, which is a combination of the electromagnetic and neutral weak isospin operators in the following way

$$
Q=T^{3}+\frac{Y}{2} .
$$

By creating this new hypercharge symmetry group $U(1)_{Y}$, the electromagnetic and weak forces become unified under the symmetry group $S U(2)_{L} \times U(1)_{Y}$ which is called the electroweak force. It is important to note that we have a symmetry group which is a product of two symmetry groups, each with their own coupling strength. The quantum numbers for the leptons and quarks in this new symmetry group are given in Table 2.1. In doing some final accounting, we find in our $S U(2)_{L} \times U(1)_{Y}$ model that we have an isotriplet of vector fields $W_{\mu}^{i}$ from $S U(2)_{L}$ with a weak isospin coupling strength $g$ and a singlet field $B_{\mu}$ with a hypercharge coupling strength $g^{\prime}$.

To get to the physically observed eigenstates of the electroweak theory, we must mix the states, $W_{\mu}^{3}$ and $B_{\mu}$, to get the observed eigenstates

$$
\begin{aligned}
& A_{\mu}=B_{\mu} \cos \theta_{W}+W_{\mu}^{3} \sin \theta_{W} \\
& Z_{\mu}=B_{\mu} \cos \theta_{W}+W_{\mu}^{3} \cos \theta_{W}
\end{aligned}
$$

The so-called weak mixing angle, $\theta_{W}$, is a parameter of the SM which represents the amount of mixing of the electromagnetic and weak forces. The weak mixing angle is a free parameter of the SM and while it is known that the electromagnetic and weak 


\section{CHAPTER 2. PHYSICS FOUNDATIONS}

\begin{tabular}{|c|c|c|c|c|}
\hline fermion & $T$ & $T^{3}$ & $Q$ & $Y$ \\
\hline \hline$\nu_{e}$ & $\frac{1}{2}$ & $\frac{1}{2}$ & 0 & -1 \\
\hline$e_{L}^{-}$ & $\frac{1}{2}$ & $-\frac{1}{2}$ & -1 & -1 \\
\hline$e_{R}^{-}$ & 0 & 0 & -1 & -2 \\
\hline$u_{L}$ & $\frac{1}{2}$ & $\frac{1}{2}$ & $\frac{2}{3}$ & $\frac{1}{3}$ \\
\hline$d_{L}$ & $\frac{1}{2}$ & $-\frac{1}{2}$ & $-\frac{1}{3}$ & $\frac{1}{3}$ \\
\hline$u_{R}$ & 0 & 0 & $\frac{2}{3}$ & $\frac{4}{3}$ \\
\hline$d_{R}$ & 0 & 0 & $-\frac{1}{3}$ & $-\frac{2}{3}$ \\
\hline
\end{tabular}

Table 2.1: Electroweak quantum numbers

forces mix, the quantity must be measured. In Chapter 5, a measurement of $\sin ^{2} \theta_{W}$ at the LHC is presented.

In this parameterization, the weak neutral current vertex interaction is of the form

$$
-i g J_{\mu}^{3}\left(W^{3}\right)^{\mu}-i \frac{g^{\prime}}{2} j-\mu^{Y} B^{\mu}=-\frac{i g}{\cos \theta_{W}} \gamma_{\mu} \frac{1}{2}\left(c_{V}^{f}-c_{A}^{f} \gamma^{5}\right)
$$

where $c_{V}^{f}=T_{f}^{3}-2 \sin ^{2} \theta_{W} Q_{f}$ and $c_{A}^{f}=T_{f}^{3}$. While this new symmetry group is a remarkable feature of the SM, all the force carriers in the model are massless. We find in nature that the neutral boson $Z^{0}$ is massive along with $W^{ \pm}$while the photon $(\gamma)$ is massless. In the $S U(2)_{L} \times U(1)_{Y}$ gauge theory, the introduction of a mass term into the formalism breaks the symmetry and is not accounted for. In order to obtain masses for the gauge bosons and fermions while retaining the gauge symmetry, we 


\section{CHAPTER 2. PHYSICS FOUNDATIONS}

discuss spontaneous symmetry breaking and the Higgs mechanism.

\section{Higgs mechanism and the SM}

While we do not discuss the Higgs mechanism in full detail in the SM, a simple example is given of mechanism by which fields are given mass. Then a description of the consequences of Higgs mechanism in the SM is given.

\section{Spontaneous symmetry breaking}

Consider a simple scalar (spinless) field with a Lagrangian

$$
\mathcal{L}=T-V=\frac{1}{2}\left(\delta_{\mu} \phi\right)^{2}-\left(\frac{1}{2} \mu^{2} \phi^{2}+\frac{1}{2} \lambda \phi^{4}\right)
$$

The Lagrangian here contains the dynamics of the system including the potential energy and kinetic energy terms ( $V$ and $T$, respectively) in a similar way as in classical physics. If $\mu^{2}>0$ the minimum ground state energy of the system is at $\phi=0$. This point obeys the reflection symmetry of the Lagrangian. Let us choose the case when $-\mu^{2}<0$ and now the potential has two minima at

$$
\phi= \pm \sqrt{\frac{-\mu^{2}}{\lambda}}= \pm v
$$

The value of the scalar field is found by perturbation around the minima such that

$$
\phi=v+\eta(x)
$$




\section{CHAPTER 2. PHYSICS FOUNDATIONS}

The idea of spontaneous symmetry breaking is that ground state lowest energy position of the system now no longer obeys the symmetry of the system. In a sense, "nature" must choose a minimal value of the system, either $+v$ or $-v$, and the choice is arbitrary.

There are examples of this in nature. For example, with ferromagnets above the Curie temperature, the electron spins are distributed randomly. However, as the temperature is lowered below the Curie temperature, the electrons spins become oriented in a uniform direction and the symmetry is the system is broken.

\section{Electroweak symmetry breaking}

The Higgs mechanism occurs when we apply the concept of spontaneously broken symmetries to the $S U(2)_{L} \times U(1)_{Y}$ electroweak symmetry group. Now, consider the SM fields now interacting in the Higgs potential similar to that described above, $V$. A detailed description of the Higgs mechanism in the SM is discussed in [12]. It has several consequeneces:

- The $S U(2)_{L} \times U(1)_{Y}$ symmetry is broken, though the $U(1)_{E M}$ symmetry is unbroken and charge generator $Q$ is conserved.

- The weak gauge bosons, $W^{ \pm}$and $Z^{0}$, gain masses while the photon remains massless. The photon is related to the weak gauge bosons via the weak mixing angle by Eq. 2.25. 


\section{CHAPTER 2. PHYSICS FOUNDATIONS}

- The fermions gain masses via spontaneous symmetry breaking of $S U(2)_{L} \times U(1)_{Y}$ as well.

- A new particle, the Higgs boson is manifest from the Higgs mechanism. It is a scalar, spin-zero, particle whose mass is a free parameter of the SM.

The success of the Higgs mechanism is that it provides a working theoretical framework for the mechanism of electroweak symmetry breaking while providing mass to the gauge bosons and fermions. As a consequence of the spin-one gauge bosons becoming massive, they gain another degree of freedom and can have a longitudinal helicity state, $\lambda=0$. We take advantage of the longitudinal polarization of the gauge bosons in our study of resonance decay kinematics to be discussed in Chapter 4. It remains to be seen whether the simple Higgs mechanism is in fact realized in nature as the Higgs boson has yet to be found. As we will see in later chapters, the search for the Higgs boson is ongoing. One of the main motivations of the following studies is to determine the nature of any new particle which we may find at the LHC and to determine if such a particle is the Higgs boson of the SM, or some indicator of other new physics. 


\section{Chapter 3}

\section{The LHC and the CMS detector}

"As of this morning we are completely wireless here at Shrute Farm, but as soon as I find out where Mose hid all the wires, we'll get all that power back on."

- Dwight K. Schrute, The Office

Discussion, thus far, has centered on the Large Hadron Collider. The LHC is opening a new regime in particle physics and providing collisions at a frequency and energy never seen before. However, the other major technical challenge for particle physicists is to detect those particles coming from high energy collisions at the LHC. The Compact Muon Solenoid experiment (CMS) is one of four particle detectors at the LHC which is collecting data for physics analysis. CMS is a multi-purpose detector designed to identify and measure the many different particles which are produced in LHC collisions. 


\section{CHAPTER 3. THE LHC AND THE CMS DETECTOR}

In the following chapter, we discuss both the LHC and CMS. The parameters of the LHC are detailed, and the general construction and the motivation for the design of the CMS detector and its early performance during commissioning with cosmic ray muons is presented.

\subsection{LHC}

The Large Hadron Collider is an accelerating ring approximately $26 \mathrm{~km}$ in circumference which collides bunches of protons. It consists of 1232 superconducting dipole magnets which keep the proton bunches in its path and r.f. (radio frequency) cavities which provide "kicks" which increase the proton energy by approximately $0.5 \mathrm{MeV}$ per turn and keep tightly bunched protons. A schematic view of the LHC is given in Fig. 3.1. In Fig. 3.1, the four particle detectors which are on the LHC accelerating ring are also illustrated where the the proton-proton beams cross and interactions take place. The instantaneous luminosity is the number of collisions per unit area per unit time usually defined in units of $\mathrm{cm}^{-2} s^{-1}$ such that the rate is defined as the instantaneous luminosity times the collision cross-section. At the LHC, it is given as [17] as

$$
\mathcal{L}=\frac{f \kappa_{B} N_{p}^{2}}{4 \pi \epsilon_{n} \beta^{*}} F
$$

where $f$ is the revolution frequency, $\kappa_{B}$ is the number of bunches, $N_{p}$ is the number of protons per bunch, $\epsilon_{n}$ is the normalized transverse emittance, $\beta^{*}$ is the betatron 


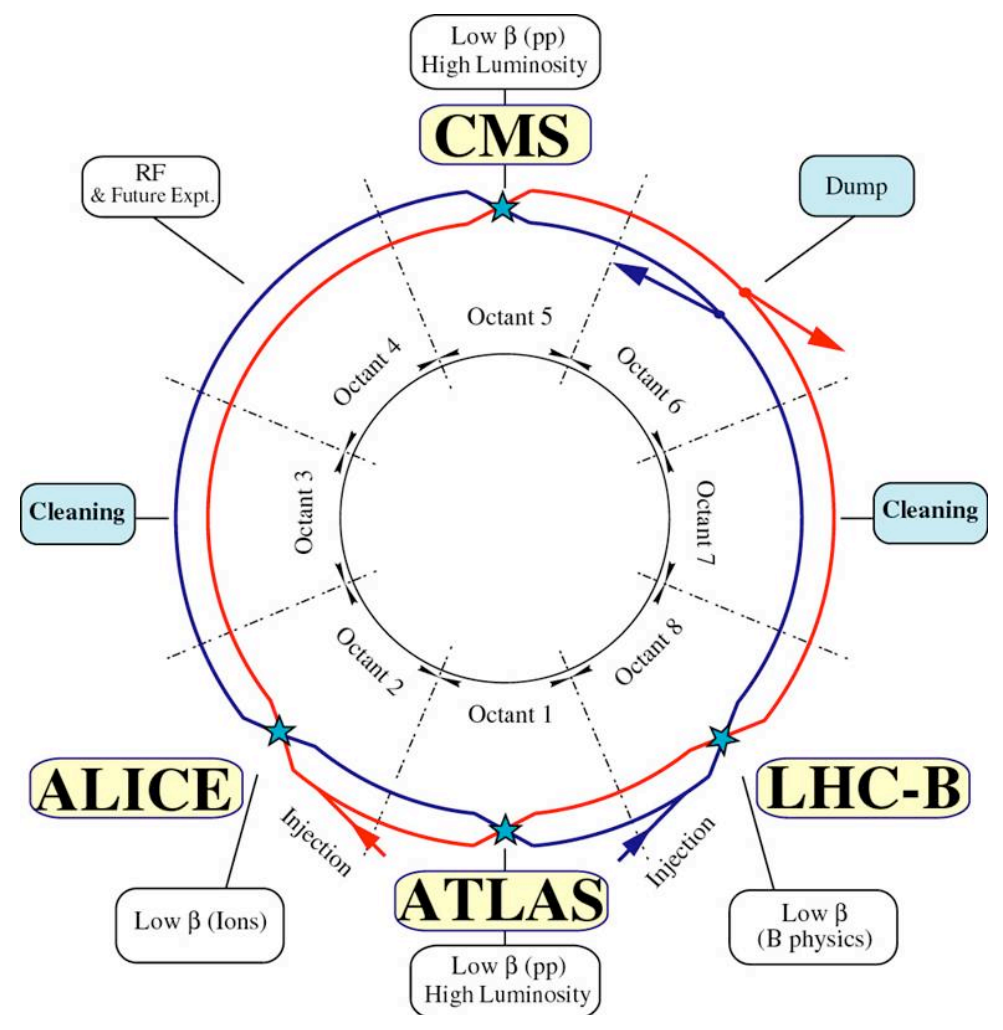

Figure 3.1: Schematic of the LHC

function at the interaction point and $F$ is the reduction factor due to the crossing angle. The factors $\epsilon_{n}$ and $\beta^{*}$ are related to the size of the proton bunches and the smaller the bunches are squeezed, the greater the luminosity increases. The emittance is a measure of the size of the beam in the transverse direction while the betatron function is a measure of the beam size in the longitudinal direction. The factor $F$ is due to the fact that beams do not collide exactly collinear, but collide with some angle.

The design instantaneous luminosity for the $\mathrm{LHC}$ is $\mathcal{L}=10^{34} \mathrm{~cm}^{-2} \mathrm{~s}^{-1}$. As a useful conversion, the integrated luminosity delivered by the LHC is often given in units of inverse barns where $1 b=10^{-24} \mathrm{~cm}^{2}$. The design energy of the LHC is 


\section{CHAPTER 3. THE LHC AND THE CMS DETECTOR}

\begin{tabular}{|c|c|c|}
\hline LHC parameter & Design & July 2011 \\
\hline \hline Energy per proton $[\mathrm{TeV}]$ & 7 & 3.5 \\
\hline Instantaneous luminosity $\left[\mathrm{cm}^{-2} s^{-1}\right]$ & $10^{34}$ & $1.75 \times 10^{33}$ \\
\hline Bunch separation $[\mathrm{ns}]$ & 25 & 50 \\
\hline Number of bunches, $\kappa_{B}$ & 2808 & 1380 \\
\hline Protons per bunch, $N_{p}$ & $1.15 \times 10^{11}$ & $1.15 \times 10^{11}$ \\
\hline$\beta^{*}[\mathrm{~m}]$ & 0.55 & 1.5 \\
\hline
\end{tabular}

Table 3.1: LHC nominal and current parameters

$7 \mathrm{TeV}$ per proton beam. So far, in 2011, the LHC machine has been running at less than design parameters with an instantaneous luminosity of $2 \times 10^{33} \mathrm{~cm}^{-2} \mathrm{~s}^{-1}$ and an energy of $3.5 \mathrm{TeV}$ per proton beam and has delivered approximately $1 \mathrm{fb}^{-1}$ of integrated luminosity by July of 2011. The nominal and 2011 beam parameters are summarized in Table 3.1. It is important to note that the current LHC parameters change rapidly and Table 3.1 is meant to give an idea of current LHC status.

The above description of the LHC parameters gives a general overview of what will be delivered to each of the detectors at the LHC. As the LHC approaches and possibly surpasses the nominal instantaneous luminosity, the number of experimental challenges that CMS will face increases. In the next section, the design of the CMS detector is discussed, including the issues of event rate and multiple interactions which 


\section{CHAPTER 3. THE LHC AND THE CMS DETECTOR}

are direct challenges presented by the LHC.

\subsection{CMS description}

CMS is a multi-purpose detector designed to detect stable and semi-stable particles produced in LHC collisions. There sub-detectors of CMS are each designed to identify and measure different classes of particles such as charged leptons like electrons and muons, photons, neutral and charged hadrons such as pions and kaons, and missing energy.

The overall layout of the CMS detector is given in Fig. 3.2. At the heart of CMS sits a 13-m-long, $5.9 \mathrm{~m}$ inner diameter, $4 \mathrm{~T}$ superconducting solenoid. Within the magnet sits the silicon tracking system, the electromagnetic calorimeter (ECAL) and the hadronic calorimeter (HCAL). Outside the solenoid are the yokes for the return magnetic field and the muon system. In order to achieve good momentum resolution within a compact spectrometer without making stringent demands on muon chamber resolution and alignment, a high magnetic field was chosen. In the bottom of Fig. 3.3 is a schematic of how the CMS detector is designed to work. Fig. 3.3 shows each CMS subdetector and illustrates how each class of (semi-)stable particle is identified.

The innermost sub-detector is the silicon tracking system which sits closest to the beam pipe. Its purpose is to detect charged particles with good momentum resolution and with high reconstruction efficiency. Further, it is required to efficiently reconstruct 


\section{CHAPTER 3. THE LHC AND THE CMS DETECTOR}

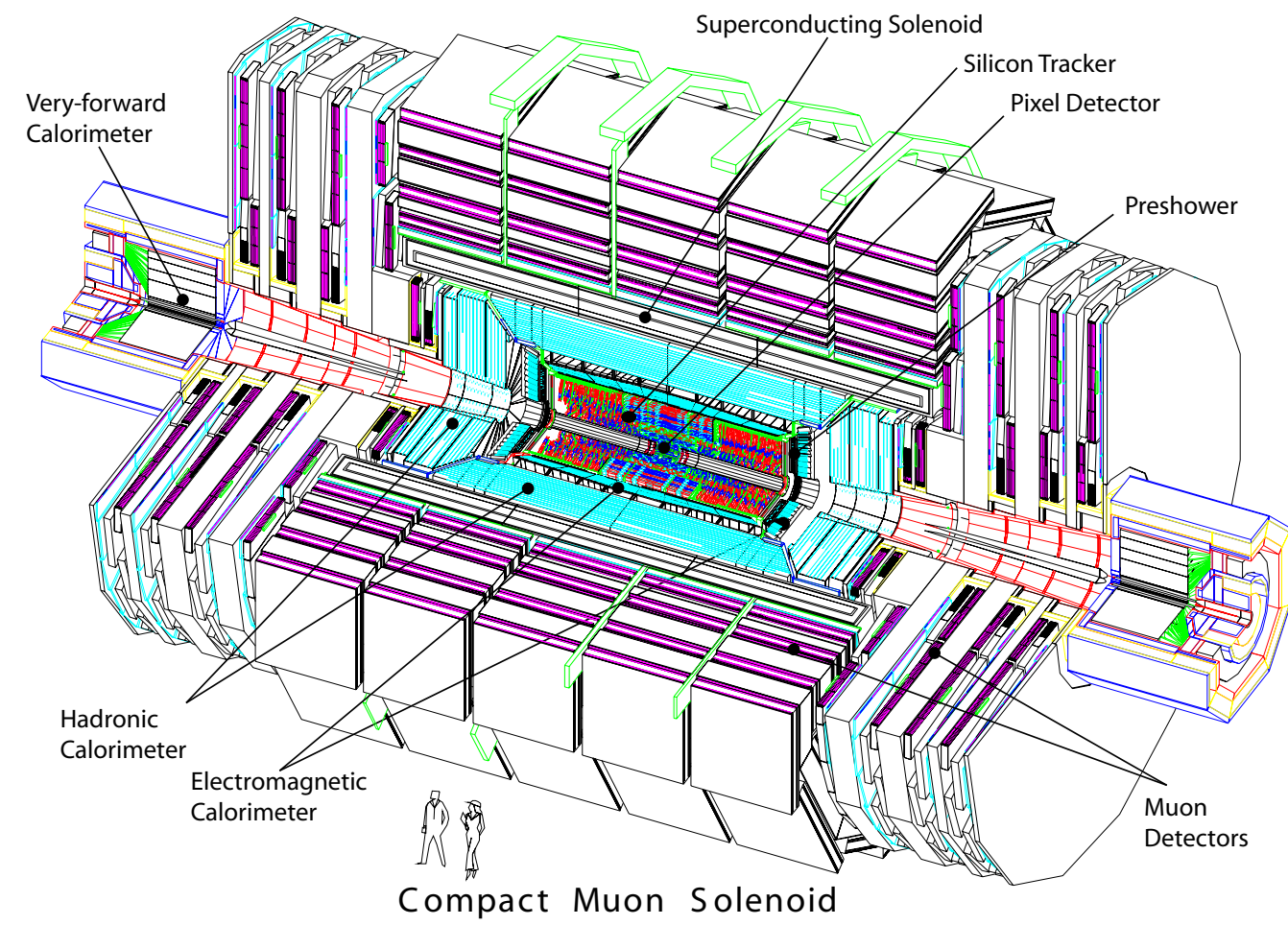

Figure 3.2: Schematic of the CMS

$\tau$ leptons and $b$-jets whose main characteristic is a displaced vertex with respect to the primary vertex. The ECAL sits outside of the tracker and is responsible for detecting photons and electrons with good energy resolution and coverage. The HCAL sits outside of the ECAL and is responsible for detecting both charged and neutral hadrons such as pions, kaons, and neutrons. It is required to have good resolution of jets and missing transverse energy, $E_{T}^{\text {miss }}$, which is defined as the missing energy in the direction perpendicular to the beam line direction ${ }^{1}$. The outer part of the HCAL, the hadron outer detector $(\mathrm{HO})$ sits outside of the coils of the solenoid. The muon system

\footnotetext{
${ }^{1}$ In general, "transverse" refers to the direction transverse to the beam line direction.
} 


\section{CHAPTER 3. THE LHC AND THE CMS DETECTOR}

is outside of the solenoid and is responsible for identifying muons and coupled with the tracking system provides good momentum resolution of muons as well.

The CMS coordinate system is defined such that the $\hat{y}$-direction is up and the $\hat{x}$-direction is towards the center of the LHC. The $\hat{z}$ direction is then defined along the beamline. In cylindrical coordinates, $\phi$ is defined as the azimuthal angle and $\theta$ as the polar angle. Though $\theta$ is sometimes used, the quantity $\eta=-\ln \tan (\theta / 2)$ is the more commonly used observable and referred to as pseudorapidity.

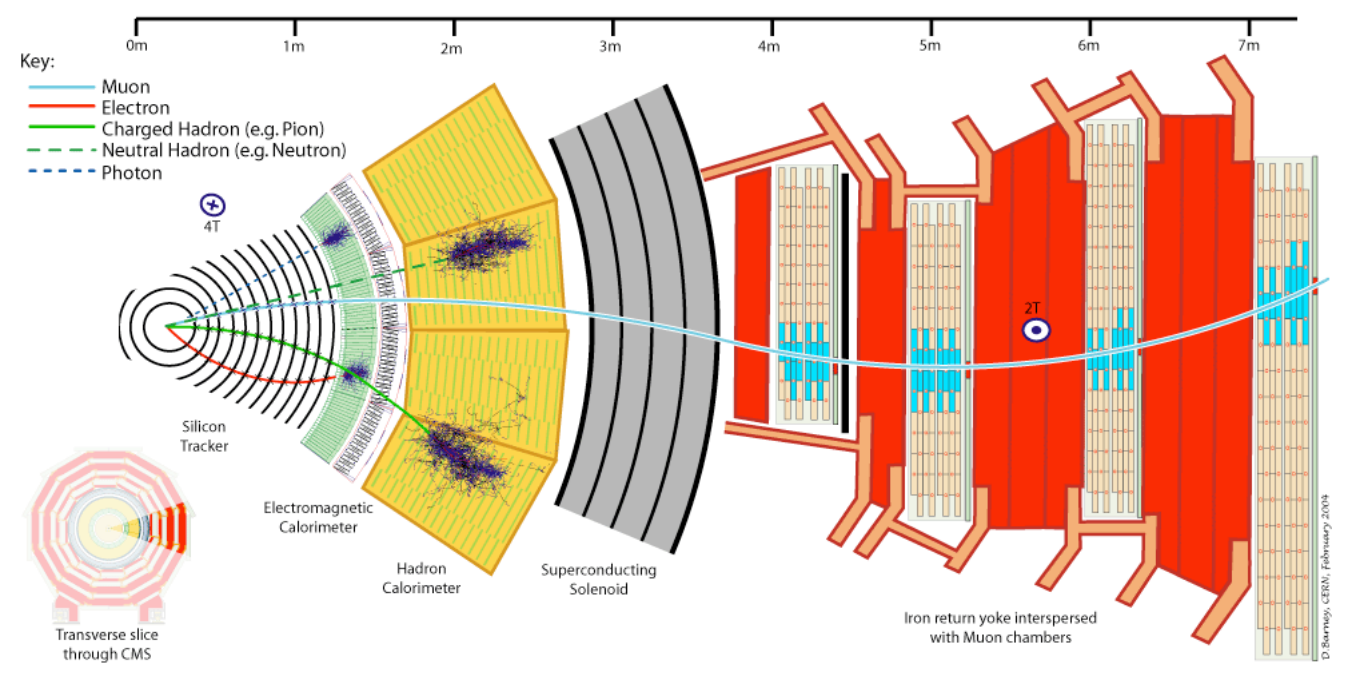

Figure 3.3: Functionality of CMS subsystems

Major experimental challenges that are addressed by CMS also include triggering and radiation damage. The LHC nominally provides bunches every $25 n s$ and thus CMS will observe approximately $10^{9}$ inelastic events per second. This event rate is much too high to store and analyze and thus an online event selection ("trigger") system is introduced to reduce the number of events saved from $10^{9}$ per second to 


\section{CHAPTER 3. THE LHC AND THE CMS DETECTOR}

approximate 100 events per second. In addition, there will be approximate 20 inelastic collisions super-imposed on the event of interest which are called pile-up events. Particles from these other collisions can be confused with those from the collision of interest and thus, high granularity detector elements with good timing resolution are required to differentiate from pile-up events. Finally, the large flux of particles coming from the interaction region leads to high radiation levels and thus, the detectors and front-end electronics must be radiation-hard.

\section{Magnet}

The CMS magnet is designed to measure muons with a momentum up to approximately $1 \mathrm{TeV}$ such that charge can be unambiguously identified. This requires a momentum resolution of about $10 \%$ in the high momentum regime. The choice of magnet for CMS was a superconducting solenoid with a magnetic field of $4 \mathrm{~T}$, a length of $12.9 \mathrm{~m}$, and inner bore radius of $5.9 \mathrm{~m}$. The length to radius ratio is determined in order to yield good momentum resolution in the forward region as well. The CMS solenoid is made with a high-purity aluminum-stabilized conductor. It consists of 2168 turns, carries a current of $20 \mathrm{kA}$ and stores approximate 2.7 GJ of energy [18]. 


\section{Inner Tracking System}

In order to attain the required granularity and precision, the inner tracking system of CMS is built with layers of silicon pixel detectors, which makes a two-dimensional measurement of the particle position in a plane, placed close to the interaction region and layers of silicon microstrip detectors, which take one-dimensional position measurements in a plane, outside of the pixel layers. The layout of the tracking system can be seen in Fig. 3.4. There are 3 layers of pixel detectors in the barrel region at

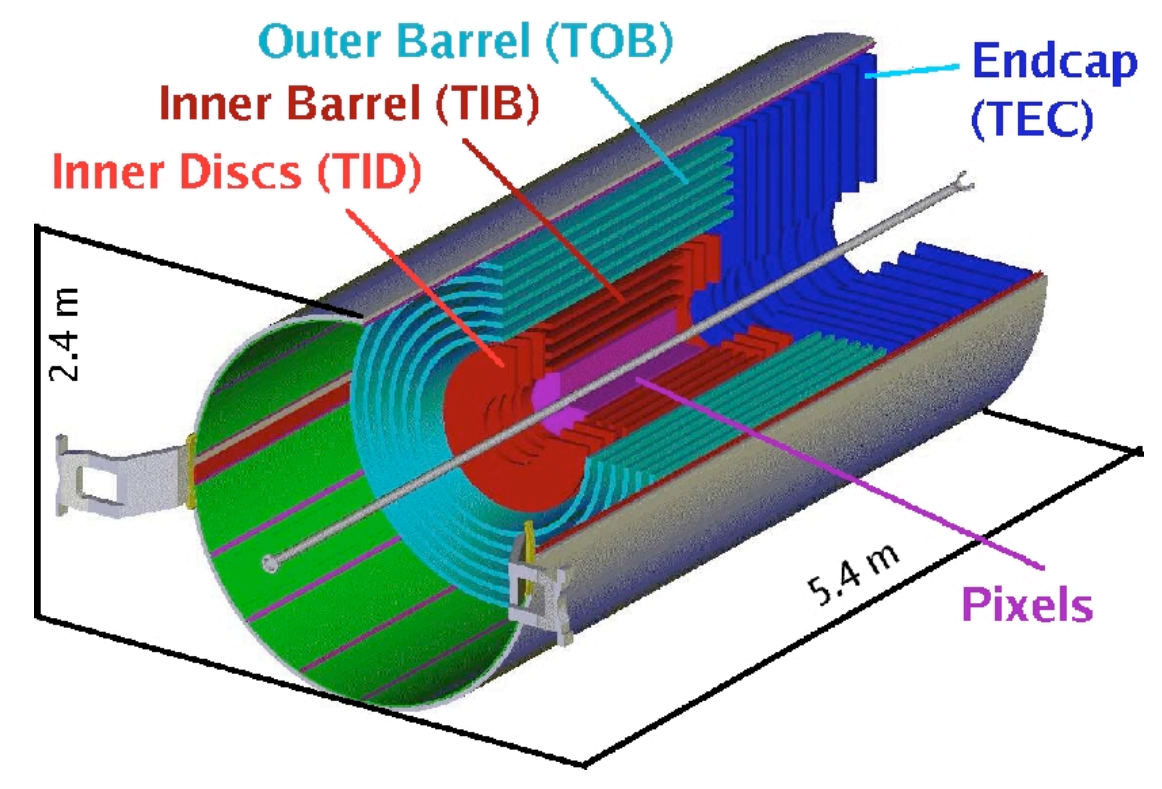

Figure 3.4: Drawing of the CMS inner tracking system

radii of 4,7 , and $11 \mathrm{~cm}$. This subsystem is called the Pixel Barrels (BPIX). In the forward region, there are 2 layers of endcap pixel detectors called the Forward Pixels (FPIX). The size of the pixels are $100 \times 150 \mu \mathrm{m}$ arranged onto modules. In the BPIX, the modules are arranged as overlapping ladders while in the FPIX the modules are 


\section{CHAPTER 3. THE LHC AND THE CMS DETECTOR}

arranged in a turbine-like structure. There are 768 pixel modules in the BPIX and 672 pixel modules in the FPIX

The silicon microstrip detector is arranged into 4 sub-detectors: the inner barrel (TIB), the outer barrel (TOB), the inner disk (TID), and the outer endcaps (TEC). The TIB is made of 4 barrel layers and the 2 closest to the interaction region are made with "stereo" modules which are two microstrip modules sitting at a stereo angle of $100 \mu$ rad with respect to each other. The stereo layers can provide measurements in $r-\phi$ and $r-z$ coordinates. Outside of the TIB is the TOB which consists of 6 layers

and the first two layers of the TOB are also made with stereo modules. The endcap layers consist of 3 TID layers and 9 TEC layers. The first 2 TID layers and layers 1,2 and 5 of the TEC consist of stereo modules. The strip pitch in all microstrip sub-detectors varies from 80 to $180 \mu \mathrm{m}$. In total, there are 15148 strip modules [18]. The tracking system provides coverage up to $|\eta|<2.4$ and contains 66 million pixels and 9.6 million silicon strips.

\section{Electromagenic Calorimeter}

The electromagnetic calorimeter (ECAL) is a hermetic and homogeneous calorimeter made of scintillating lead tungstate $\left(\mathrm{PbWO}_{4}\right)$ crystals with coverage in pseudorapidity out to $|\eta|<3.0$. The barrel region (EB) contains 61200 crystals with 7234 crystals in each of the endcaps (EE). The crystals have a short radiation length where $X_{0}=0.89 \mathrm{~cm}$ and short Moliere lengths of $2.2 \mathrm{~cm}$. The crystals are fast and emit $80 \%$ 


\section{CHAPTER 3. THE LHC AND THE CMS DETECTOR}

of their light within $25 \mathrm{~ns}$ and are radiation-hard. In order to detect the relatively low light yield, photodetectors able to operate in high magnetic field are used to detect the light. The scintillating light is detected by silicon avalanche diodes (APDs) in the barrel and vacuum phototriodes (VPTs) in the endcaps [18].

In the $E B$, there are 36 identical "supermodules" that cover the region from $0<$ $|\eta|<1.479$. The crystals have a cross-section of $22 \times 22 \mathrm{~mm}^{2}$ and a length equal to 25.8 $X_{0}$. The EE cover a region from $1.479<|\eta|<3.0$. The crystals in the EE have a cross-section of $28.6 \times 28.6 \mathrm{~mm}^{2}$ and a length equal to $24.7 X_{0}$.

\section{Hadronic Calorimeter}

The hadronic calorimeter (HCAL) consists of four parts: the Hadron Barrel (HB), the Hadron Endcap (HE), the Hadron Outer (HO) and the Hadron Forward (HF). It is designed to provide good energy resolution and provide good containment for the $E_{T}^{\text {miss }}$ measurement.

The $\mathrm{HB}$ and $\mathrm{HE}$ are made of thin plastic scintillator tiles alternating with brass plates which are used as the absorber material. Brass is used because it is easy to machine, has a short interaction length, and is non-magnetic. The scintillating light is converted by wavelength-shifting fibers which are then detected by hybrid photodiodes (HPDs). There are also stainless steel plates used as some of the absorbing layers to structurally support the HB and HE. The HB consists of 2304 towers covering the region of $|\eta|<1.4$ with segmentation in the $\eta-\phi$ plane of $0.087 \times 0.087$. The HE 


\section{CHAPTER 3. THE LHC AND THE CMS DETECTOR}

consists of 2304 towers in the pseudorapidity region $1.3<|\eta|<3.0$ with segmentation in $\eta$ of 0.087 and varying from $5^{\circ}$ to $10^{\circ}$ in $\phi$.

The HF covers the pseudorapidities from $3.0<|\eta|<5.0$ and provides full geometric coverage for measurement of the missing energy. It is constructed of steel absorbers and quartz fibers and the Cherenkov light in the quartz fibers is detected by photomultipliers. There are 900 towers in total which segmentation in $\eta$ of 0.175 and $\phi$ of $10^{\circ}$. The $\mathrm{HO}$ sits outside of the solenoid and is effectively a "tail-catcher" in the central region of $|\eta|<1.26$ consisting of plastic scintillators. The $\mathrm{HO}$ effectively increases the hadron calorimetry to greater than 10 interaction lengths and has the same geometry in $\eta$ and $\phi$ as the HB [18].

\section{Muon Detector System}

The CMS muon system is designed to identify, trigger, and measure muon momentum efficiently. Three types of gaseous detectors are used to detect the muons. The muon momentum is measured both in the muon system and the inner tracking system where the muon system uses the bending angle leaving solenoid and the inner tracking system uses the track curvature fit. By combining the inner tracking information with the muon system measurements, a global fit of the muon momentum is determined and gives better accuracy in the full momentum range than either measurement alone. The inner tracking system dominates the momentum measurement until approximately $200 \mathrm{GeV}$, and above, the muon system improves the momentum measurement in the 


\section{CHAPTER 3. THE LHC AND THE CMS DETECTOR}

global fit. The layout of the muon system is given in Fig. 3.5.

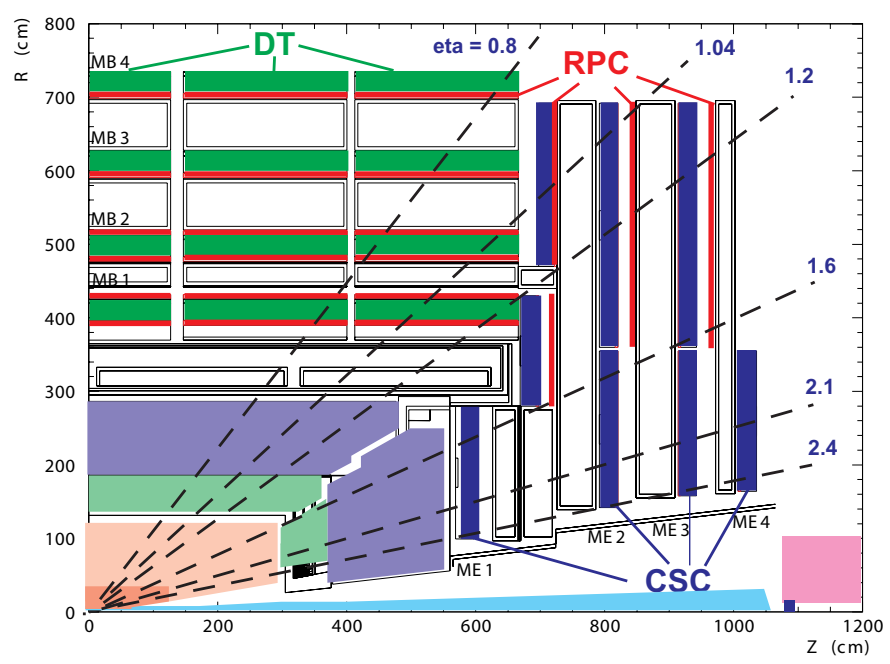

Figure 3.5: CMS muon system

In the barrel region from $|\eta|<1.2$ where low muon rates are expected, drift tube (DT) chambers are used to measure the muons tracks. The DTs consist of four stations alternating with the iron return yokes for the magnetic field. In the first three stations, there are 12 drift tubes chambers in each with 4 measuring the $r-\phi$ plane, then 4 measuring the $z$ plane, then 4 more measuring the $r-\phi$ plane again. In the fourth station, there are no drift tubes measuring the $z$ plane. The single-point resolution is approximately $200 \mu m$.

In the endcap region, cathode strip chambers (CSC) are used due to the higher muon rate and higher backgrounds. They cover the $\eta$ region out to 2.4. Each CSC is trapezoidal in shape and consists of 6 gas gaps, each gap having a plane of radial cathode strips and a plane of anode wires running almost perpendicular to the strips. 


\section{CHAPTER 3. THE LHC AND THE CMS DETECTOR}

The spatial resolution from each chamber is approximately $200 \mu \mathrm{m}$.

There are also resistive plate chambers (RPC) throughout both the barrel and endcaps that cover a region of $|\eta|<2.1$. The RPCs provide good time resolution for muon triggering and identification although their positional resolution is coarser than either the DTs or CSCs [18].

\section{Trigger and data acquisition}

As stated above, the nominal LHC bunch crossing results in approximately $10^{9}$ collisions per second while only approximately 100 events can be stored per second. Each event size is approximately 1.5 MB for $p p$ interactions; thus, a high rejection rate is required. The CMS trigger and data acquisition system consists of the detector electronics, the Level-1 trigger processors, the readout network, and the online processor farm that executes the software for the High-Level trigger.

The Level-1 trigger is required to perform trigger calculations in less than $1 \mu s$. Thus, custom hardware processors are used to perform the Level-1 decision. It makes decisions based on primitive objects such as photons, electrons, muons, and jets above some transverse energy $E_{T}$ or transverse momentum $p_{T}$ as well as global sums of $E_{T}$ or $E_{T}^{\text {miss }}$. The design rate for the Level- 1 trigger is $100 \mathrm{kHz}$ and is set by the average time to transfer full detector information throughout the readout system [18]. The High-Level trigger (HLT) is required to reduce the Level-1 output from $100 \mathrm{kHZ}$ to $100 \mathrm{~Hz}$. In doing so, the HLT software utilizes partial reconstruction and many 
CHAPTER 3. THE LHC AND THE CMS DETECTOR

virtual trigger levels (calorimeter and muon system information followed by tracking data, then the full detector information) to achieve the desired rejection rate [18].

\subsection{Commissioning and Tracker Alignment}

Before the first collisions from the LHC arrived, CMS was able to collect cosmic ray muons for commissioning of the full detector. With this cosmic ray data, CMS performed many vital studies to calibrate and understand the detector. Because of this opportunity, CMS was able to quickly produce several physics results with the first $40 \mathrm{pb}^{-1}$ of data acquired in 2010 from the LHC. Several commissioning studies were completed to better understand the detector performance from trigger performance studies [19] to pixel [20] and microstrip [21] calibration to mapping of the CMS magnetic field [22] and many others [23].

In particular, the alignment of the inner tracking system during the commissioning period was of great importance and is highlighted in this section. The alignment of the tracker has strong relationships to many physics results and is related to many aspects of detector performance such as momentum scale and resolution of charged leptons, tracking efficiency and $b$-quark tagging. In Chapter 5 we will take a particular example of the effect of tracker alignment in the measurement of the weak mixing angle $\sin ^{2} \theta_{W}$ which was defined in Section 2.3. 


\section{CHAPTER 3. THE LHC AND THE CMS DETECTOR}

\section{Introduction to tracker alignment}

The CMS collaboration conducted a month-long data-taking exercise known as the Cosmic Run At Four Tesla (CRAFT) during October-November 2008 with the goal of commissioning the experiment for extended data-taking [23]. This first run to collect cosmic ray data with the full CMS detector was critical for startup ${ }^{2}$. With all installed detector systems participating, CMS recorded 270 million cosmic-ray-triggered events with the solenoid at its nominal axial field strength of $3.8 \mathrm{~T}$. Of all the data taken during this time period, only a few percent of the cosmic muons cross the tracker volume and are used in the alignment of the tracker; the total amount of tracks used in tracker alignment is approximately 3 million. Data with no magnetic field was also taken with the solenoid off and this data was used to cross-check results with fieldon data. The distribution of momentum $p$, azimuthal angle $\phi$, and polar angle $\theta$ for cosmic rays in data is given in Fig. 3.6 and compared with simulation as well. Good agreement is observed between data and simulation.
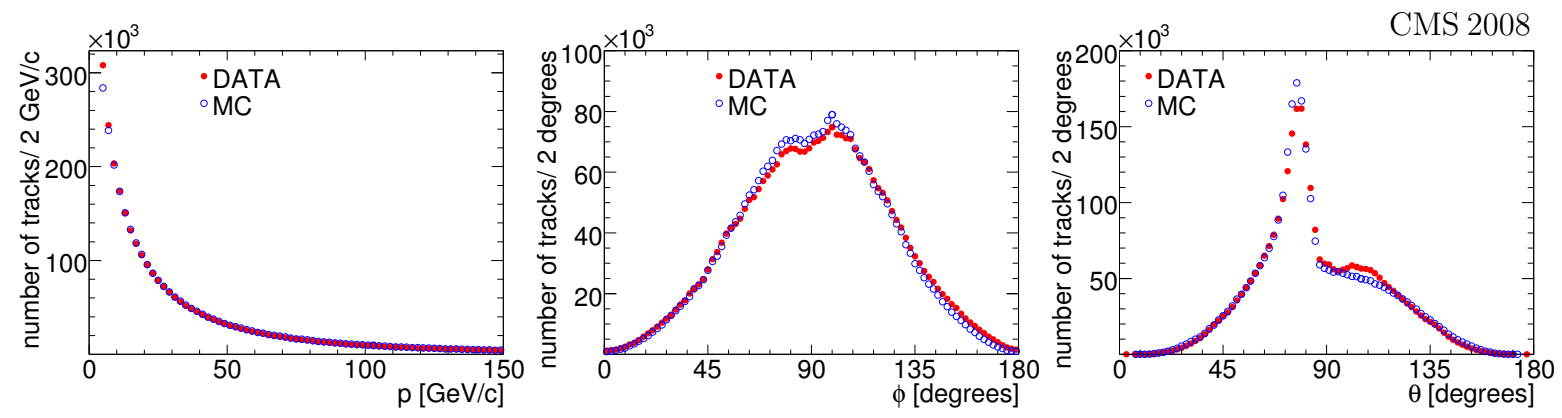

Figure 3.6: Distribution of cosmic ray muons in data and simulation

\footnotetext{
${ }^{2}$ Cosmic ray data is currently collected regularly, but CRAFT was the first instance.
} 


\section{CHAPTER 3. THE LHC AND THE CMS DETECTOR}

During the commissioning of the CMS detector, alignment of the tracker was performed using cosmic ray muons to improve tracking performance before the arrival of first collisions from the LHC. As was mentioned above, the aim of the tracker is to measure the trajectories of charged particles (tracks) with excellent momentum, angle, and position resolution, and with high pattern recognition efficiency. The job of tracker alignment is to precisely determine the position of all silicon modules. Recall that the tracker consists of 1440 2D pixel modules and 15148 1D strip modules. It is a challenging task and one of the critical aspects for achieving the design track parameter resolutions. In the context of alignment, position is used to refer both to the location of the center point of active areas on modules and to the orientation of active areas. The hit position resolution is in the range 10 to $30 \mu \mathrm{m}$ and therefore the alignment precision should be better than $10 \mu \mathrm{m}$ to achieve optimal track parameter resolution. Simulation studies based on the design (ideal) tracker geometry imply an impact parameter resolution of about $15 \mu \mathrm{m}$ and a transverse momentum $\left(p_{T}\right)$ resolution of about $1.5 \%$ for $100 \mathrm{GeV} / c$ muons [24].

The overall strategy of tracker alignment is to use all possible inputs to best constrain the positions of the modules. It is a combination of optical survey information taken before and during installation of the tracker and cosmic track information in situ. The goal of the track-based alignment procedures is to determine the module positions from a large sample of reconstructed charged particle trajectories. Each trajectory is built from charge depositions ("hits") on individual detectors, assuming a piece-wise 


\section{CHAPTER 3. THE LHC AND THE CMS DETECTOR}

helical track model, incorporating effects from multiple scattering and energy loss. The "Combinatorial Track Finder" (CTF) track algorithm [21] was used to reconstruct the cosmic muon trajectories and results in five track parameters describe the helical trajectory of a track at the point closest to the nominal interaction point: distance of closest approach in the transverse $d_{x y}$ and longitudinal $d_{z}$ directions, track azimuthal angle $\phi$, track polar angle $\theta$, and transverse momentum $p_{T}$. Optical surveys taken during module construction and integration provide initial alignment parameters for many of the modules. Additionally, the survey information was used as a constraint in the alignment procedure, as will be described below.

The final output from the alignment procedure is a geometry which records the positions and orientations of all the strip and pixel modules. Further, the procedure also produces alignment position errors for each aligned detector element, called the Alignment Position Errors (APEs), which is the estimated uncertainty on the module position in the three global coordinates The track reconstruction uses the APEs added in quadrature to the hit errors during the track reconstruction procedure. This allows for efficient track reconstruction in the presence of misalignment and a correct pull distribution of track parameters. The APEs are also of great importance when combining tracker information with muon system information in the global fit of the full muon track to properly weight the information from the inner tracker and muon subsystems. The determination of APEs is described in more detail below. 


\section{Track-based alignment algorithms and strategy}

The alignment is an optimization problem that can be formulated in the context of linear least squares. Corrections to the module position ("alignment parameters") p are determined by minimizing an objective function

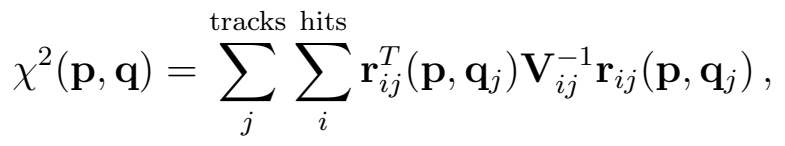

which can be expressed as the sum over all hits $i$ on all tracks $j$ and track parameters $\mathbf{q}_{j}$, assuming negligible correlations between hits. Track residuals $\mathbf{r}_{i j}=\mathbf{m}_{i j}-\mathbf{f}_{i j}\left(\mathbf{p}, \mathbf{q}_{j}\right)$ are defined as the difference between the measured hit position $\mathbf{m}_{i j}$ and the trajectory impact point $\mathbf{f}_{i j}$, both of which are given in the module local coordinate system. The residuals are either one- or two-dimensional vectors, depending on the type of module, and $\mathbf{V}_{i j}$ is either the squared error or the covariance matrix in case of one- or twodimensional residuals, respectively. A visualization of the residual in two-dimensions is given in Fig. 3.7.

Two statistical methods were employed to solve the alignment problem. The global alignment algorithm ("Millepede II") [25] minimizes the $\chi^{2}$ function in Eq. 3.2 by taking into account track and alignment parameters simultaneously. For the $n$ alignment parameters $\mathbf{p}$ of interest, the problem is reduced to the solution of a matrix equation of size $n$. The value of $n$ is of the order of $10^{5}$ for six degrees of freedom of 16588 modules. The covariance matrix $\mathbf{V}_{i j}$ is approximated by a diagonal matrix with uncertainties $\sigma_{i j}$ for uncorrelated hit measurements $\mathbf{m}_{i j}$ of the track $j$. Given reasonable starting values, 


\section{CHAPTER 3. THE LHC AND THE CMS DETECTOR}

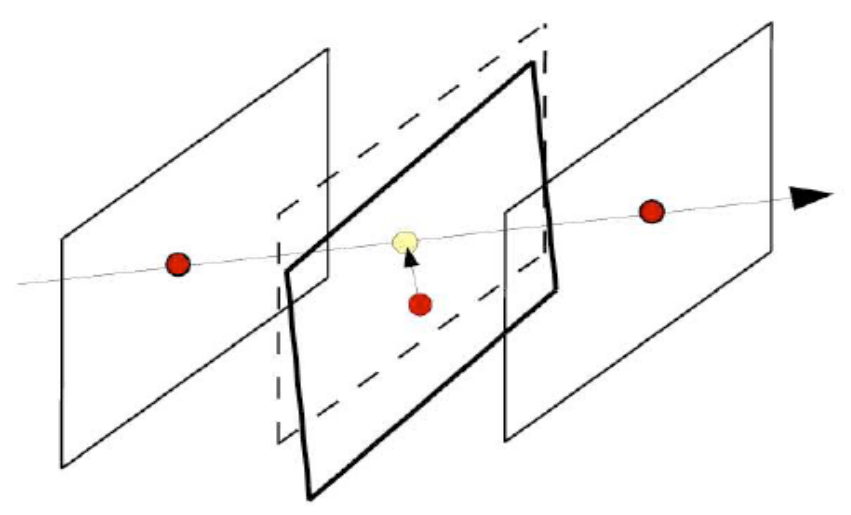

Figure 3.7: Visualization of track residuals

$\mathbf{p}_{0}$ and $\mathbf{q}_{j 0}$, the track model prediction can be linearized. Since angular corrections are small, the linearized problem is a good approximation for alignment.

The local iterative algorithm ("Hits and Impact Points") [26] approximates Eq. 3.2 by assuming no track parameter $\mathbf{q}$ dependence and therefore ignores correlations between alignment parameters for different modules in one iteration. The track parameters and correlations between different modules are taken into account through iterations of the minimization procedure and refitting the tracks with new alignment constants after each iteration. This approach allows for a simplification of the formalism and leads to a solution of the six-dimensional matrix equation for the six alignment parameters of each module in each iteration.

The local iterative algorithm permits the inclusion of survey measurements in the formalism of Eq. 3.2, as described in Ref. [27]. This leads to an additional term in the 


\section{CHAPTER 3. THE LHC AND THE CMS DETECTOR}

$\chi^{2}$ function to be minimized independently for each module in a given iteration:

$$
\chi_{m}^{2}(\mathbf{p})=\sum_{i}^{\text {hits }} \mathbf{r}_{i}^{T}(\mathbf{p}) \mathbf{V}_{i}^{-1} \mathbf{r}_{i}(\mathbf{p})+\sum_{j}^{\text {survey }} \mathbf{r}_{* j}^{T}(\mathbf{p}) \mathbf{V}_{* j}^{-1} \mathbf{r}_{* j}(\mathbf{p}),
$$

where the original formulation has been modified to include survey information from the $k$ hierarchial levels for each sub-detector. The track residuals $\mathbf{r}_{i}(\mathbf{p})$ do not have explicit dependence on track parameters and enter the sum over hits in a given module $m$. The six-dimensional survey residuals $\mathbf{r}_{* j}$ are defined as the difference between the reference and the current sensor position. The survey measurement covariance matrix $\mathbf{V}_{* j}$ reflects both the survey precision and additional uncertainties due to changes in the detector. These errors can be configured differently for different hierarchy levels based on how much the position and orientation of certain structures are known to vary in time.

During the CRAFT alignment analysis, implementations in the CMS software of the global and local methods were applied in combination. The strength of the global method was solving effectively the global correlations, while for the local method it was that the same track fit was used as in the standard CMS reconstruction, and that survey information could easily be incorporated thus allowing for alignment with more degrees of freedom. In order to take advantage of both methods, the final alignment parameters were produced starting from the output of the global method analysis, then further aligning the tracker with the local method. The resulting combined method geometry was the final result from the alignment procedure which took advantage of the the strengths of both algorithms. 
CHAPTER 3. THE LHC AND THE CMS DETECTOR

\section{Results and validation from cosmic ray alignment}

Several approaches were employed to validate the alignment results. The lowlevel quantities that were used in the $\chi^{2}$ minimization, such as residuals and the $\chi^{2} /$ ndf of the tracks, were monitored and used to extract the APEs. Given the limited size of the alignment track sample, it was used both for alignment and validation. In addition, a high-level validation by splitting cosmic tracks was performed to monitor the track parameter resolution. Moreover, techniques for comparing positions of modules within differently derived geometries allow for a better understanding of the alignment performance.

\section{APEs and Residuals}

In the track refit the APEs for each hit were assumed to be the same in the three spatial directions. For an alignment performed using cosmic ray tracks, the precision of the alignment varies within a given sub-detector because of the different illumination of modules due to their orientation relative to cosmic rays. The radius of the sphere representing the APE for each module, $r_{\text {sphere, }}$ was therefore taken to be $r_{\text {sphere }}=r_{0} / \sqrt{N_{\text {entries }}}$, where $N_{\text {entries }}$ represents the number of hits in a module. The value of $r_{0}$ was chosen in order to have the Gaussian standard deviation of the distribution of the residuals normalized to their error approximately equal to one in the symmetric interval covering $95 \%$ of the distribution. The value of $r_{0}$ was defined at the sub-detector level. In the case of small or zero $N_{\text {entries, }}$ a precision compatible with 


\section{CHAPTER 3. THE LHC AND THE CMS DETECTOR}

survey and assembly data was used. APEs were calibrated using the set of alignment parameters obtained from the combined method. The RMS of the distribution of the median of the residuals (DMR) are given in Table 3.2 and illustrate the inputs in the determination of the APEs. They are presented for the non-aligned (before), global, local, and combined geometries. The values from simulation (MC) are also presented and show good agreement. The number of modules with greater than 30 hits is also presented to illustrate the typical number of tracks passing through certain modules. Examples of the distribution of the normalized residuals after the calibration of the APEs are shown in Fig. 3.8.

Table 3.2: RMS of the distribution of the median of the residuals (DMR)

\begin{tabular}{|c|c|c|c|c|c|c|c|}
\hline & $\begin{array}{c}\text { before } \\
{[\mu \mathrm{m}]}\end{array}$ & $\begin{array}{c}\text { global } \\
{[\mu \mathrm{m}]}\end{array}$ & $\begin{array}{c}\text { local } \\
{[\mu \mathrm{m}]}\end{array}$ & $\begin{array}{c}\text { combined } \\
{[\mu \mathrm{m}]}\end{array}$ & $\begin{array}{c}\text { combined } \\
\mathrm{MC}[\mu \mathrm{m}]\end{array}$ & $\begin{array}{c}\text { ideal } \\
\mathrm{MC}[\mu \mathrm{m}]\end{array}$ & $\begin{array}{c}\text { modules } \\
>30 \text { hits }\end{array}$ \\
\hline BPIX $\left(u^{\prime}\right)$ & 328.7 & 7.5 & 3.0 & 2.6 & 2.1 & 2.1 & $757 / 768$ \\
BPIX $\left(v^{\prime}\right)$ & 274.1 & 6.9 & 13.4 & 4.0 & 2.5 & 2.4 & \\
FPIX $\left(u^{\prime}\right)$ & 389.0 & 23.5 & 26.5 & 13.1 & 12.0 & 9.4 & $393 / 672$ \\
FPIX $\left(v^{\prime}\right)$ & 385.8 & 20.0 & 23.9 & 13.9 & 11.6 & 9.3 & \\
TIB $\left(u^{\prime}\right)$ & 712.2 & 4.9 & 7.1 & 2.5 & 1.2 & 1.1 & $2623 / 2724$ \\
TOB $\left(u^{\prime}\right)$ & 168.6 & 5.7 & 3.5 & 2.6 & 1.4 & 1.1 & $5129 / 5208$ \\
TID $\left(u^{\prime}\right)$ & 295.0 & 7.0 & 6.9 & 3.3 & 2.4 & 1.6 & $807 / 816$ \\
TEC $\left(u^{\prime}\right)$ & 216.9 & 25.0 & 10.4 & 7.4 & 4.6 & 2.5 & $6318 / 6400$ \\
\hline
\end{tabular}

All tracks used in the validation procedure were refitted with APEs corresponding to the alignment parameters obtained with the combined method. To avoid a bias in the determination of the hit residuals, the track prediction was calculated without information from the hit under consideration. The track $\chi^{2} /$ ndf distribution is shown in Fig. 3.9. Overall, there is significant improvement in the track reconstruction going 

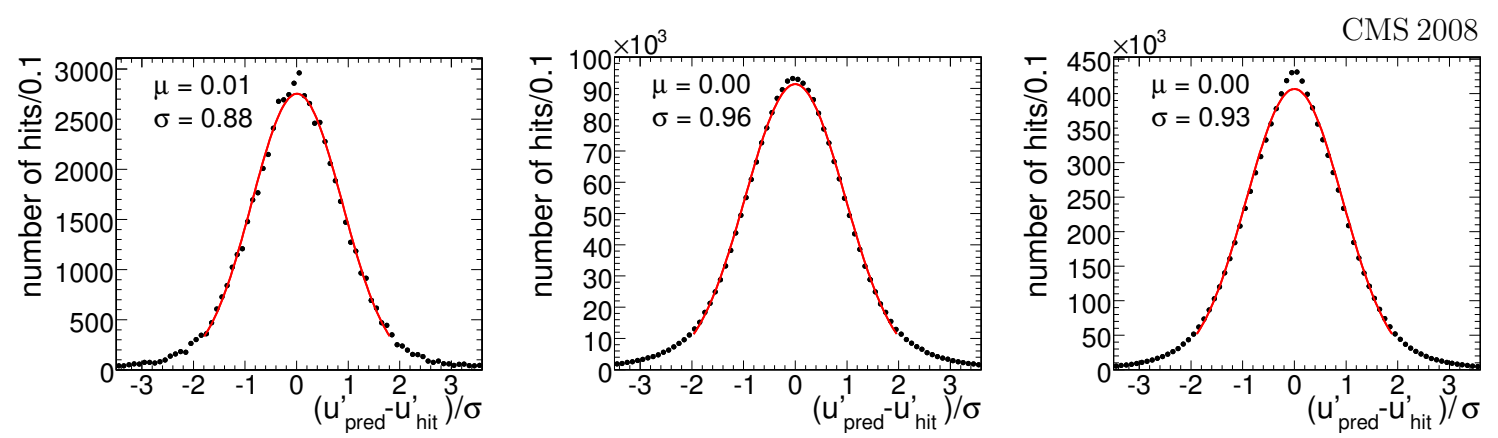

Figure 3.8: Normalized residuals for various geometries

from the geometry without any alignment, to the alignment using tracks with the local and the global method, and finally to the combined result. These values are in agreement with the expected statistical precision as determined using simulated events. They are also comparable to values obtained from a MC simulation based on the ideal detector geometry.

\section{Track parameter resolution}

The track parameter resolutions can be validated with independent reconstruction of the upper and the lower portions of cosmic ray tracks and comparison of track parameters at the point of closest approach to the nominal beamline. A schematic of this procedure is shown in Fig. 3.10. Both the upper and lower track segments were required to have at least three pixel hits. This mimics the topology of collision tracks. The track segments were reconstructed independently. Figure 3.11 shows the difference between upper and lower portions of tracks for all five track parameters. 


\section{CHAPTER 3. THE LHC AND THE CMS DETECTOR}

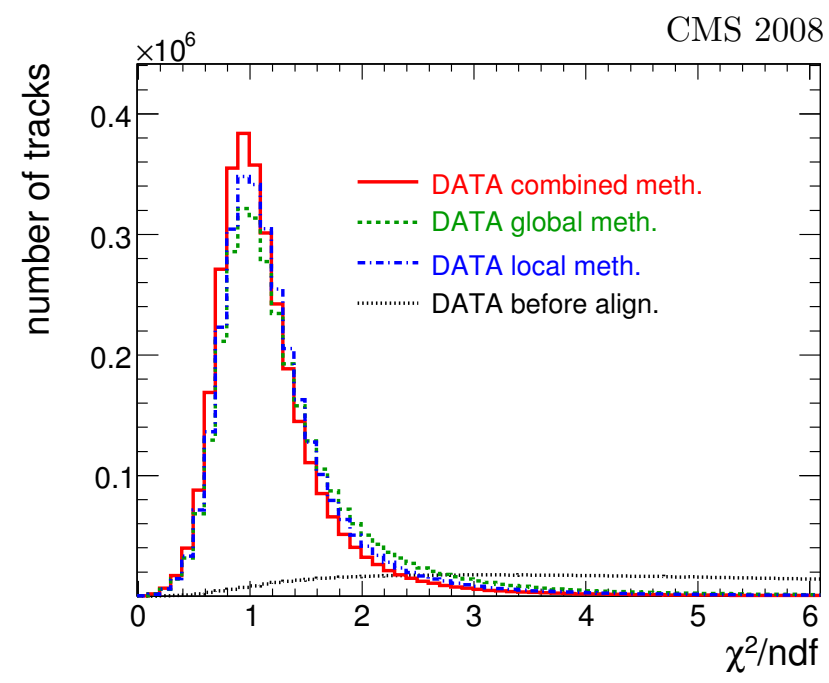

Figure 3.9: Track $\chi^{2} /$ ndf for various geometries

Distributions are shown for the distance of closest approach in the transverse direction $d_{x y}$ (top left), the same in the longitudinal direction $d_{z}$ (top right), the track azimuthal angle $\phi$ (middle left), the track polar angle $\theta$ (middle right), and $1 / p_{T}$ (bottom left). Results are shown for four geometries: data before alignment (black dotted lines), data with combined method alignment (red solid), combined method MC (green dashed), and ideal MC (blue dash-dotted). There is significant improvement due to tracker alignment, with good agreement between data and MC simulations. The results of the combined method are approaching those of a MC simulation with ideal detector geometry. The $p_{T}$ measurements are most sensitive to the strip part of the tracker, while the other four parameters are dominated by the alignment of the pixel detector. The plot on the bottom right shows the $1 / p_{T}$ difference normalized to its error, that is 


\section{CHAPTER 3. THE LHC AND THE CMS DETECTOR}

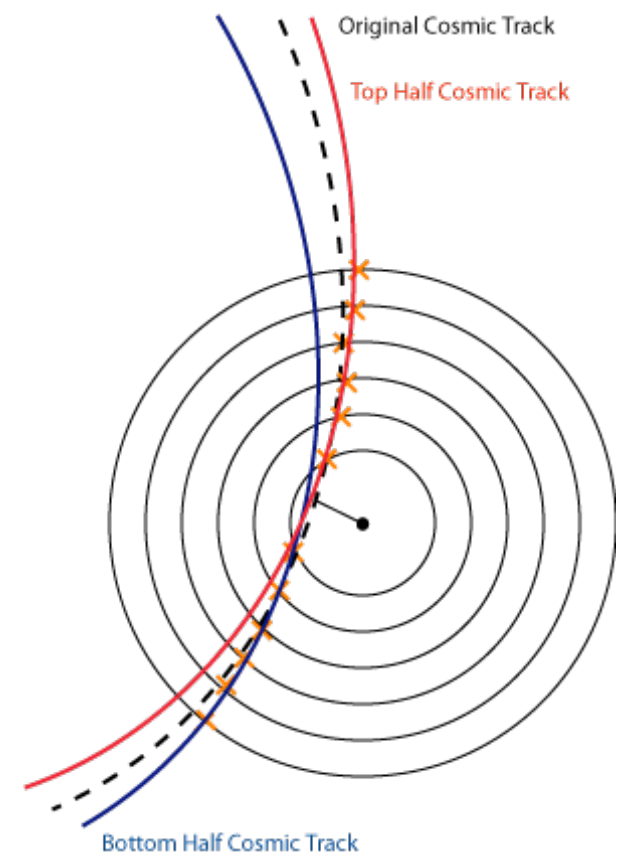

Figure 3.10: Schematic of cosmic track splitting procedure.

$\left(1 / p_{T, 1}-1 / p_{T, 2}\right) / \sqrt{\sigma_{1 / p_{T}, 1}^{2}+\sigma_{1 / p_{T}, 2}^{2}}$. The normalized distribution shows that the error estimates on the track parameters are in good agreement with predictions from $M C$ simulations.

Figure 3.12 shows the dependence of the resolution on $p_{T}$ for the track parameters $d_{x y}$ and $1 / p_{T}$. The RMS of the distribution truncated at $95 \%$ is quoted at each momentum interval, shown for the distance of closest approach in the transverse direction $d_{x y}$ (left) and for $1 / p_{T}$ (right). Results are shown for the geometries derived from ideal MC (blue squares), and from the alignment result with cosmic ray data (red circles) and combined method MC (green triangles). 


\section{CHAPTER 3. THE LHC AND THE CMS DETECTOR}
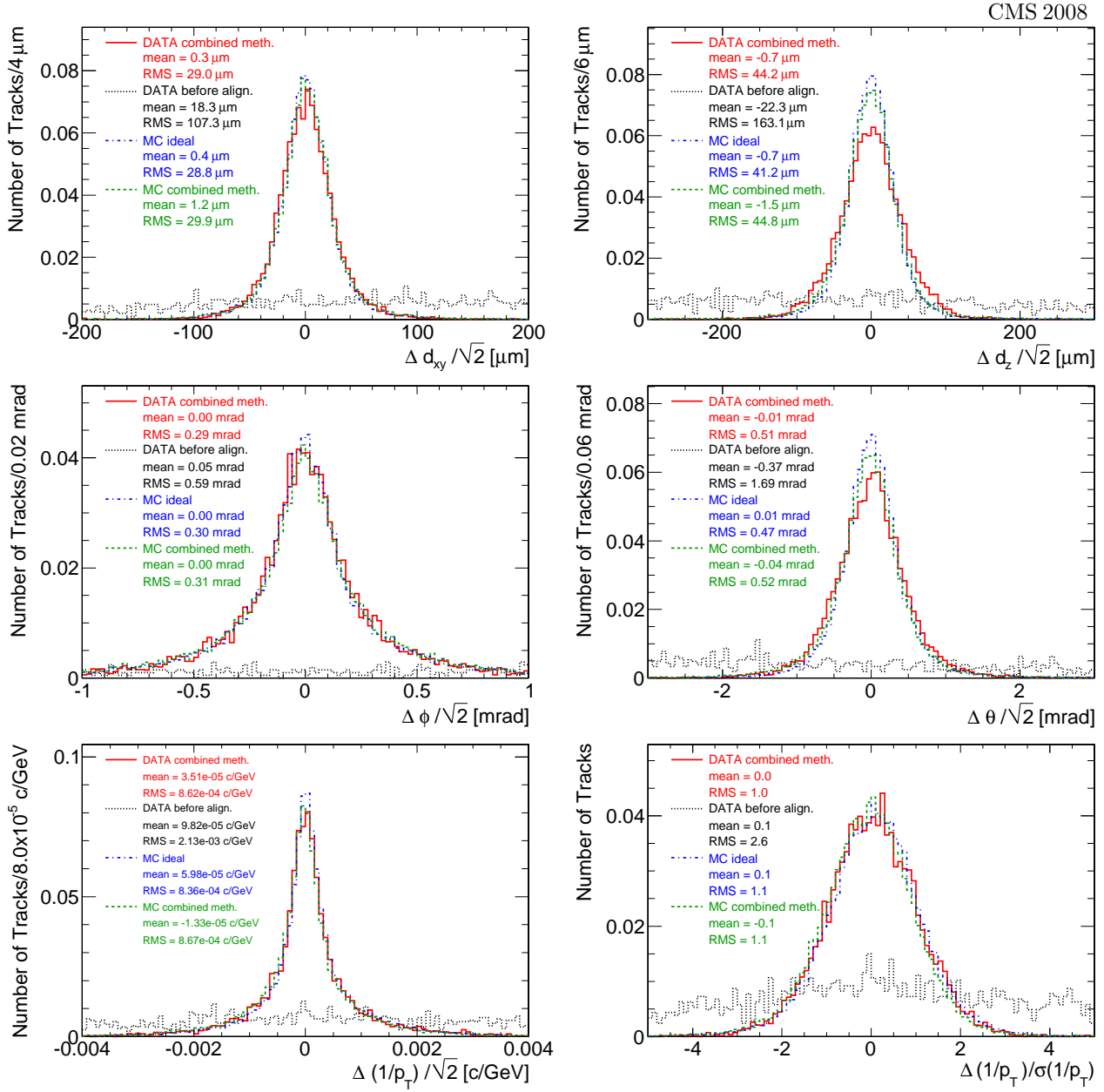

Figure 3.11: Track parameter resolution using split cosmic tracks

\section{Detector geometry studies and $\chi^{2}$-invariant deformations}

Comparisons between geometries (i.e. non-aligned, global, local and combined geometries) are utilized as another validation tool for module positions. They provide visualization of the movements of detector elements and also are used a validation cross-check that the module positions are reasonable and contain no weak modes. Weak modes are $\chi^{2}$-invariant geometrical modes which alignment may be insensitive 


\section{CHAPTER 3. THE LHC AND THE CMS DETECTOR}
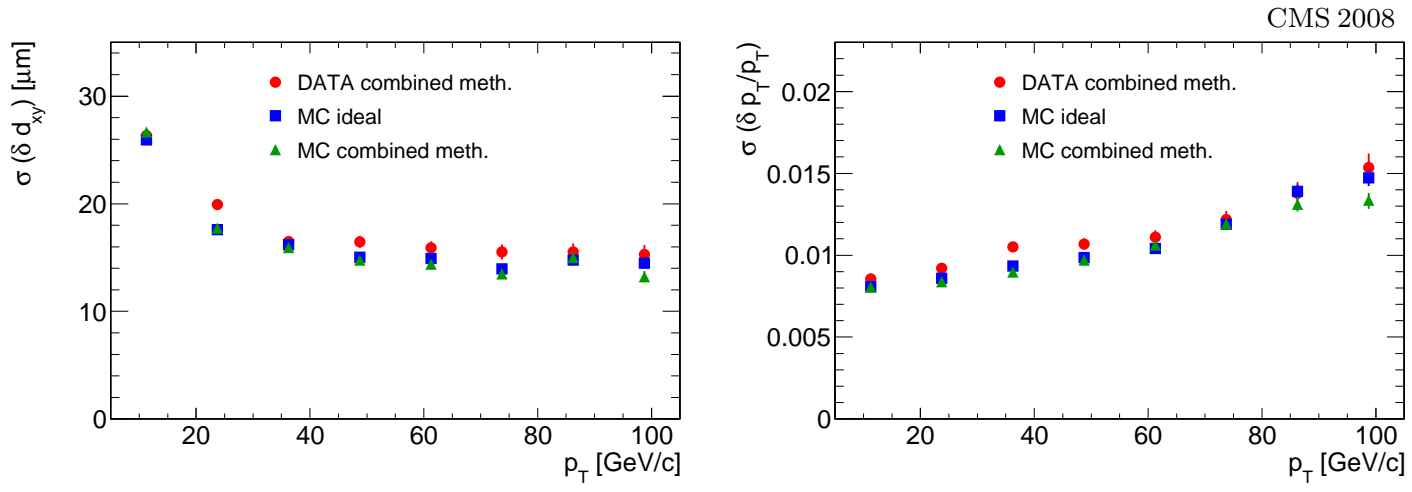

Figure 3.12: Transverse impact parameter and $p_{T}$ resolution as a function of $p_{T}$

to and degrade tracking performance; they are discussed below in more detail. A comparison between two geometries is done after correcting for an overall residual shift and rotation of the whole detector, or a sub-detector, with respect to its centerof-gravity.

Using the geometry obtained with the combined method, a comparison with the design geometry indicates that the two BPIX half-barrels are shifted along the vertical axis by about $0.4 \mathrm{~mm}$ and the two half-barrels of the TIB have an extra separation along the $z$ axis of about $5 \mathrm{~mm}$. Both displacements are mechanically possible. The large displacement of the TIB half-barrels is supported by the optical survey measurements described in Ref. [28]. Figure 3.13 shows the displacement in the $y$ - and in the $z$ coordinates of the BPIX and TIB modules with respect to the design position. In the case of the TIB, a large scatter of the $z$-coordinate of the modules with respect to their design position is observed. The modulation of the displacement as a function 


\section{CHAPTER 3. THE LHC AND THE CMS DETECTOR}
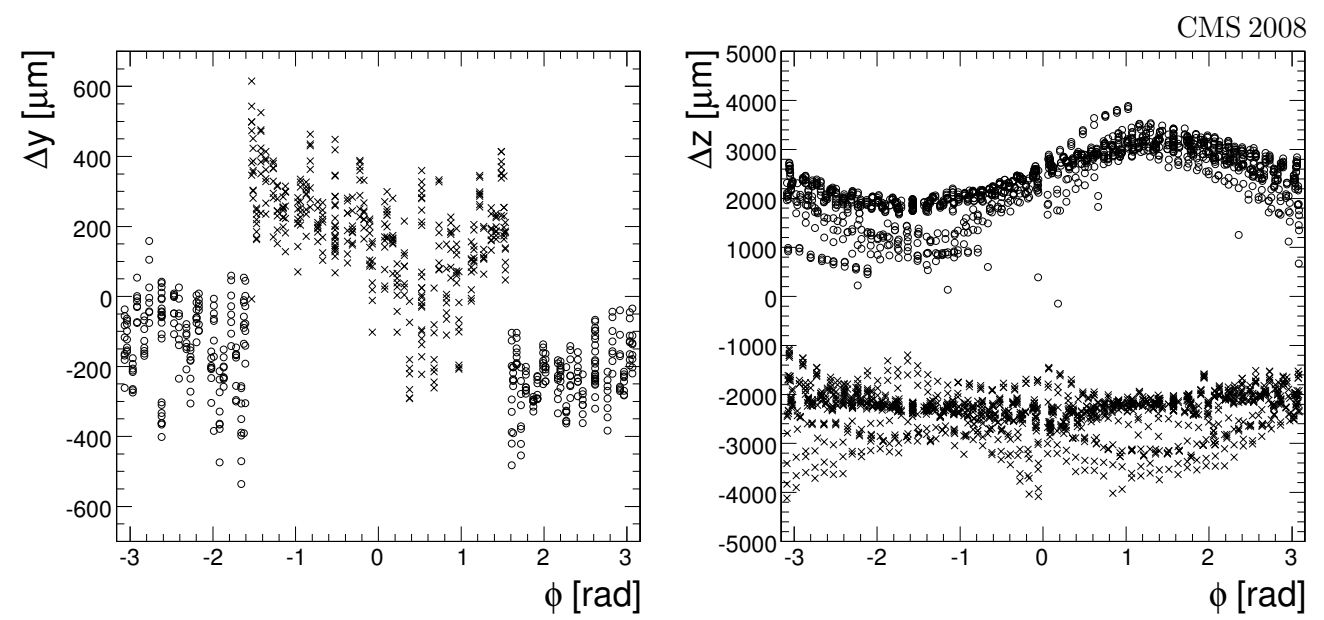

Figure 3.13: Comparison of module positions between combined and design geometries

of the $\phi$ position of the module can be explained by the presence of the weak mode skew in the combined geometry, as discussed in the previous section.

A global translation and rotation of the whole tracker is an example of a trivial transformation which leaves the $\chi^{2}$ value of Eq. 3.2 unchanged. This transformation has no effect on the internal alignment and is easily resolved by a suitable convention when defining the global reference frame. The convention that the center-of-gravity of all modules coincides with the design position was used in this work. A similar convention was used for the rotation of the tracker.

There are, however, non-trivial transformations, so-called "weak" modes, that also preserve the $\chi^{2}$ value of Eq. 3.2 and are of larger concern. The presence of weak modes in the geometry resulting from track-based alignment was investigated following the approach described in Ref. [27]. Nine cylindrical systematic distortions, in $\Delta r, \Delta \phi$, and $\Delta z$ as a function of $r, \phi$, and $z$ were applied to the aligned geometry. Studies were 


\section{CHAPTER 3. THE LHC AND THE CMS DETECTOR}

performed with both global and local alignment methods and the results presented here were obtained with the global method. The systematically misaligned geometries were used as a starting point and the alignment procedure was repeated. The nine geometries obtained after the alignments were then compared to the original aligned geometry to see if the distortions can be recovered by the alignment procedure.

The results were analyzed separately for the pixel (BPIX and FPIX), barrel strip (TIB and TOB), and forward strip (TID and TEC) sub-detectors. The remaining displacements of the modules in the TIB and TOB, which are expected to have the best illumination from cosmic ray tracks, are shown in Fig. 3.14 for four systematic deformations: the layer rotation $\left(\Delta \phi=c_{1}+c_{2} r\right)$, the twist deformation $\left(\Delta \phi=c_{1} z\right)$, the skew $\left(\Delta z=c_{1} \cos \phi\right)$, and the $z$-expansion $\left(\Delta z=c_{1} z\right)$. The layer rotation can be well recovered in alignment with cosmic ray tracks, while the scatter in the module position difference is an evidence for weak modes in other projections. The twist and the skew deformations are reflected to a lesser degree in track $\chi^{2}$, and therefore are only partially recovered. Finally, as the distribution of the track $\chi^{2}$ changes only marginally with respect to the z-expansion, this deformation cannot be recovered using cosmic ray tracks only.

In the future, additional information with tracks from LHC beam interactions, which should be uniform in $\phi$, and with additional constraints from the verticies and the masses of resonances, may provide better sensitivity to those systematic deformations. 
CHAPTER 3. THE LHC AND THE CMS DETECTOR

\section{Outlook}

The first alignment of the full CMS tracker is presented using cosmic muon data. In order to use as much information as possible, a combination of optical survey information and cosmic tracks are used to constrain module positions. The general strategy and concept of tracker alignment and an introduction to track-based alignment at CMS using the global and local algorithms is discussed. A significant improvement in track residuals, track $\chi^{2} /$ ndof, and track parameters is observed between the aligned geometries and the design geometry. In particular, the track parameter validation provides a first measurement of tracking performance at CMS. An investigation of the detector geometry and $\chi^{2}$-invariant modes are also shown.

Since the LHC has been providing collision data for CMS, tracker alignment is now performed with both collision tracks and cosmic ray data. Many of the foundations of tracker alignment at CMS evolved during the CRAFT exercise and are in practice in the current alignment of the detector. 


\section{CHAPTER 3. THE LHC AND THE CMS DETECTOR}
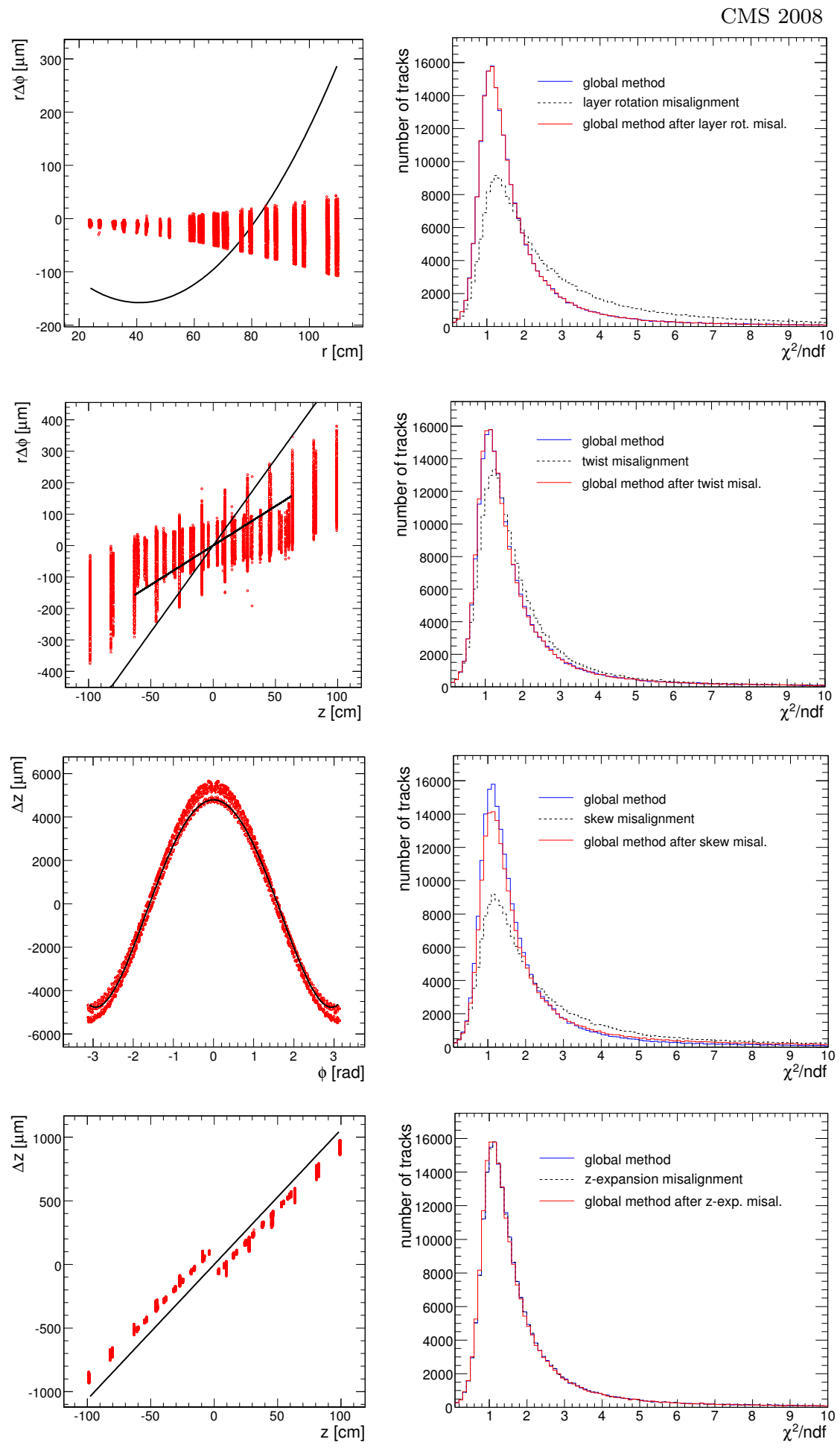

Figure 3.14: Geometrical deformation and track $\chi^{2}$ performance in weak mode alignment 


\section{Chapter 4}

\section{Kinematics of Generic Resonances}

"Life is like a grapefruit ... Well, it's sort of orangy-yellow and dimpled on the outside, wet and squidgy in the middle. It's got pips inside, too. Oh, and some people have a half a one for breakfast." - Ford Prefect to Creature

"So Long and Thanks for All the Fish", Douglas Adams

In previous chapters, we mentioned the Higgs mechanism and the search for the yet unfound Higgs boson. At the LHC, we hope to find the nature of electroweak symmetry breaking whether it be via the Higgs mechanism or otherwise. However, in Sec. 1.2, we also discussed the many motivations and possibilities for physics beyond the SM. If we find a resonance at the $\mathrm{LHC}$, its nature will be unknown at the time of discovery. It could be a Higgs boson, but it could also be one of many possible hypothesized new particles. The Higgs boson is a spin-zero particle that remains unchanged under the parity operation, a scalar particle. However, a new resonance 


\section{CHAPTER 4. KINEMATICS OF GENERIC RESONANCES}

could be another spin-zero particle, perhaps with odd parity, called a pseudoscalar particle. Such a particle may show up in supersymmetric models where the number of possible Higgs particles is increased. Perhaps the new resonance could be a new gauge boson of spin-one, a vector or axial-vector resonance, which show up in extradimensional models [29]. It is even possible that a new resonance could be of spin-two nature, a tensor particle, which could show up in Randall-Sundrum warped extradimensional models as a graviton [30] [31]. In addition to the above examples, there are many other possibilities for beyond the SM resonances, very exotic things such as Hidden Valley models [32] and even yet unhypothesized possibilities. In searches for new physics, we hope to be unbiased towards any of these hypothesized models in determining the nature of any new resonance.

In this chapter, we approach the interactions of a generic resonance of spin-zero, spin-one, or spin-two with the SM fields in a model independent way with as few theoretical constraints as possible. By determining the generic decay kinematics of a given resonance, we can derive the angular distributions for any signal hypothesis. By using the decay angular distributions of the final state SM particles of a new resonance, we hope to understand the spin, parity, and interactions of any newly discovered resonance. In this chapter, the formalism is developed to analytically describe resonance decay kinematics [33]. Then, in Chapter 6, we will introduce the techniques for distinguishing between such various hypotheses. 


\section{CHAPTER 4. KINEMATICS OF GENERIC RESONANCES}

\subsection{Resonances at the LHC}

We examine the types of resonances that can be produced at the LHC via protonproton collisions. The production and decay mechanisms for a hypothetical colorand charge-neutral particle $X$ of possible spin-zero, -one or -two are outlined below. Though other spins are possible, we consider the most elementary cases. Such a particle can be produced in two main channels at the LHC: gluon fusion, $g g \rightarrow X$, and quark-antiquark annihilation, $q \bar{q} \rightarrow X$. As an example, in Fig. 4.1 [34], the partonparton luminosities are shown for the $\mathrm{LHC}$ at $14 \mathrm{TeV}$. The green line corresponds to the $g g$ luminosity; the red line corresponds to the the $q \bar{q}=\sum_{i}\left(q_{i} \bar{q}_{i}+\bar{q}_{i} q_{i}\right)$ luminosity; and the green line corresponds to the $q g$ luminosity ${ }^{1}$. The distributions for these partonparton luminosities come from parton distributions functions which are discussed in greater detail in Sec. 5.1.

The Feynman diagrams for these production mechanisms are shown in Fig. 4.2. The left and middle diagrams in Fig. 4.2 are for gluon fusion, either at tree-level or through a loop, and the right diagram is for quark-antiquark annihilation. In the case of gluon fusion production, the resonance $X$ can be only spin-zero or spintwo because the coupling of a spin-one particle to two massless spin-one particles (gluons) is forbidden $[35,36]$. The massless spin-one gluons will have a helicity state of \pm 1 and so the polarization of $X$ particle is constrained to be either 0 or \pm 2 . In the case of quark-antiquark annihilation, in the limit of light quarks we consider the

\footnotetext{
${ }^{1}$ we ignore $q g$ production since we consider only colorless and chargeless resonances
} 


\section{CHAPTER 4. KINEMATICS OF GENERIC RESONANCES}

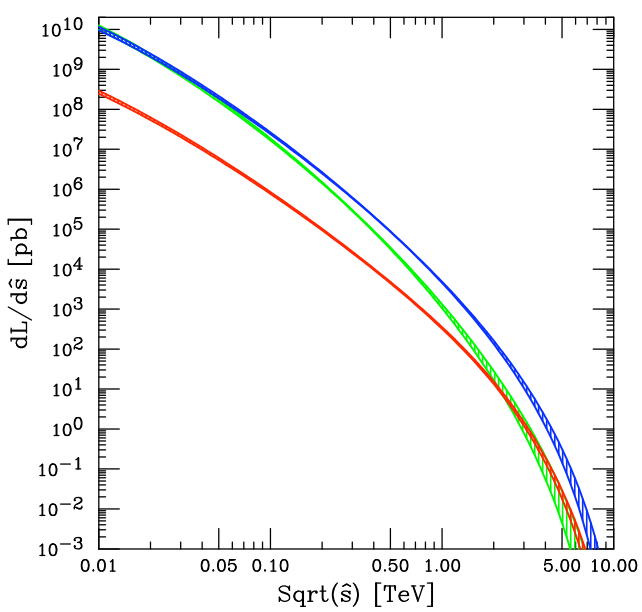

Figure 4.1: Parton-parton luminosities at the LHC

chiral symmetry to be a "good" quantum number where we have purely $\mathrm{LH}$ or $\mathrm{RH}$ interactions. When the chiral symmetry is exact, the polarization of the particle $X$ can then only be \pm 1 . Thus, a particle $X$ produced via $q \bar{q}$ annihilation can only be spin-one or spin-two as a spin-zero particle cannot have a polarization of \pm 1 . The above is summarized in Table 4.1. Analogous to Fig. 4.2, the decay channels of a particle $X$ to the SM fields are given in Fig. 4.3. The left and middle diagrams of
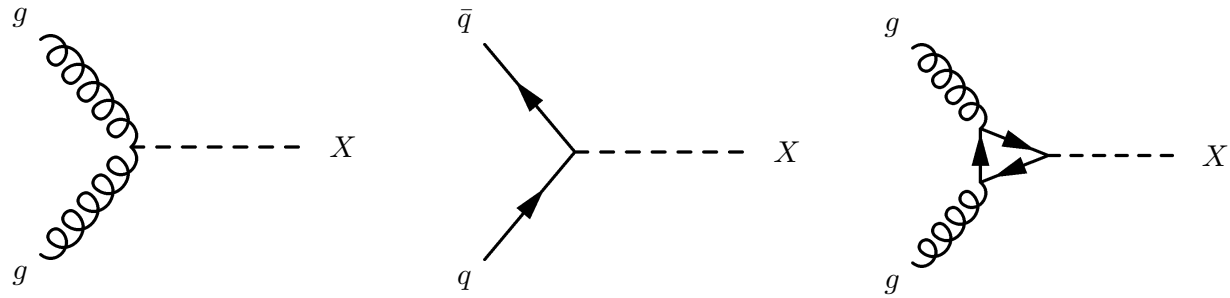

Figure 4.2: Production mechanisms for particle $X$ at the LHC 


\section{CHAPTER 4. KINEMATICS OF GENERIC RESONANCES}
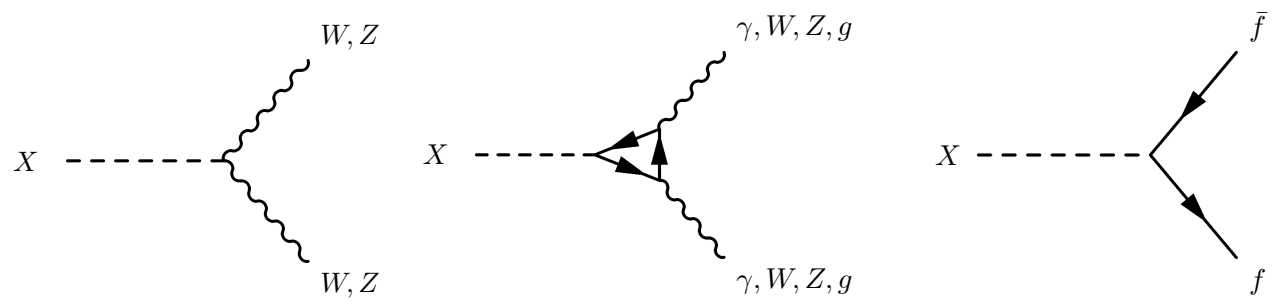

Figure 4.3: Decay channels for particle $X$ at the LHC

\begin{tabular}{|c|c|c|c|}
\hline Resonance spin & production mechanism & polarization & SM decay channels \\
\hline \hline spin-zero & $g g$ & 0 & $\gamma \gamma, W^{+} W^{-}, Z Z, g g$ \\
\hline spin-one & $q \bar{q}$ & \pm 1 & $W^{+} W^{-}, Z Z, f \bar{f}$ \\
\hline spin-two & $g g$ or $q \bar{q}$ & $0, \pm 1, \pm 2$ & $\gamma \gamma, W^{+} W^{-}, Z Z, g g, f \bar{f}$ \\
\hline
\end{tabular}

Table 4.1: Summary of resonance production and decay at the LHC

Fig. 4.3 show decay at tree level and via a loop to the gauge bosons and the right diagram shows decays to fermions. The constraints from the Landau-Yang theorem and massless relativistic fermions from the production case remain valid. This means that the a spin-zero particle $X$ decaying to fermions is highly suppressed in the limit when the fermions are massless. Also, a spin-one particle does not couple spin-one massless particles such as photons or gluons. The possibilities are summarized in Table 4.1. 


\section{CHAPTER 4. KINEMATICS OF GENERIC RESONANCES}

\subsection{General interactions with SM fields}

In this section, the interaction of a color- and charge-neutral exotic particle $X$ with two spin-one bosons $V$ (such as gluons, photons, $Z$, or $W$ bosons) or a fermionantifermion pair (such as leptons or quarks) is summarized [33]. The spin of $X$ can be zero, one, or two. The most general amplitudes are constructed consistent with Lorentz invariance and Bose-symmetry, as well as gauge-invariance with respect to unbroken subgroups of $S U(3) \times S U(2)_{L} \times U(1)_{Y}$ of the $\mathrm{SM}$.

In the description of the general interactions below, we use the following definitions. The four-momentum of the particle $X$ is denoted by $q$ and the four-momenta of the gauge bosons or fermions by $q_{1,2}$. The polarization vectors of gauge bosons are denoted by $\epsilon_{1,2} ;$ we assume them to be transverse $q_{i} \epsilon_{i}=0$. Fermion wave functions are conventional Dirac spinors, $u$ and $v$. We employ the field strength tensor of a gauge boson [12] with momentum $q_{i}$ and polarization vector $\epsilon_{i}$ as $f^{(i), \mu \nu}=\epsilon_{i}^{\mu} q_{i}^{\nu}-\epsilon_{i}^{\nu} q_{i}^{\mu}$, and the conjugate field strength tensor as $\tilde{f}_{\mu \nu}^{(i)}=1 / 2 \epsilon_{\mu \nu \alpha \beta} f^{(i), \alpha \beta}=\epsilon_{\mu \nu \alpha \beta} \epsilon_{i}^{\alpha} q_{i}^{\beta}$. We use $\tilde{q}=q_{1}-q_{2}$ to denote the particular combination of the momenta of the two final state particles. 


\section{CHAPTER 4. KINEMATICS OF GENERIC RESONANCES}

\section{Spin-zero $X$ to gauge bosons}

Consider a particle $X$ which is of spin-zero. The general couplings of a spin-zero particle to two gauge bosons are written as

$$
A(X \rightarrow V V)=v^{-1} \epsilon_{1}^{* \mu} \epsilon_{2}^{* \nu}\left(a_{1} g_{\mu \nu} M_{X}^{2}+a_{2} q_{\mu} q_{\nu}+a_{3} \epsilon_{\mu \nu \alpha \beta} q_{1}^{\alpha} q_{2}^{\beta}\right)
$$

Here, the couplings constants are denoted as $a_{1}, a_{2}$ and $a_{3}$. These coupling constants are in general dimensionless complex valued numbers and are the free components of the interaction which vary depending on the nature of the new physics model. The even parity terms are $a_{1}$ and $a_{2}$ while $a_{3}$ corresponds to an odd-parity coupling. The four-momenta of the outgoing bosons are denoted by $q_{1}$ and $q_{2}$ and their polarization vectors are denoted as $\epsilon_{1}$ and $\epsilon_{2}$. The momenta of the two $V^{\prime}$ 's are parameterized as $q_{1,2}=\left(m_{X} / 2,0,0, \pm \beta m_{X} / 2\right)$, where $\beta=\left(1-4 m_{V}^{2} / m_{X}^{2}\right)^{1 / 2}$ is the velocity of gauge bosons in the $X$ rest frame. As an example, in the case of the SM Higgs boson at leading order, we set $a_{1} \neq 0$ and $a_{2}=a_{3}=0$.

For a spin-one gauge boson the polarization vectors have the values $\pm 1,0$ and can be written as

$$
\begin{aligned}
& e_{1,2}^{\mu}(0)=M_{V}^{-1}\left( \pm \beta M_{X} / 2,0,0, M_{X} / 2\right), \\
& e_{1}^{\mu}( \pm)=e_{2}^{\mu}(\mp)=\frac{1}{\sqrt{2}}(0, \mp 1,-i, 0) .
\end{aligned}
$$

These polarization vectors are the manifestation of the decay daughter helicity states of a spin- $J$ particle from Section 2.2 


\section{CHAPTER 4. KINEMATICS OF GENERIC RESONANCES}

\section{Spin-one $X$ to gauge bosons}

Next, we consider the case when an exotic particle $X$ has spin one and arbitrary parity. As a consequence of the Landau-Yang theorem, the spin-one particle $X$ cannot interact with two massless identical gauge bosons. The phenomenology of spin-one decays into two $Z$ bosons was recently discussed in Ref. [29]. Following that reference, we consider the amplitude for the decay to two identical massive gauge bosons $X \rightarrow$ $Z Z$ which is dependent on two independent couplings

$$
A(X \rightarrow Z Z)=g_{1}^{(1)}\left[\left(\epsilon_{1}^{*} q\right)\left(\epsilon_{2}^{*} \epsilon_{X}\right)+\left(\epsilon_{2}^{*} q\right)\left(\epsilon_{1}^{*} \epsilon_{X}\right)\right]+g_{2}^{(1)} \epsilon_{\alpha \mu \nu \beta} \epsilon_{X}^{\alpha} \epsilon_{1}^{*, \mu} \epsilon_{2}^{*, \nu} \tilde{q}^{\beta} .
$$

Similar to the spin-zero case, $g_{1}^{(1)}$ and $g_{2}^{(1)}$ are dimensionless effective complex coupling constants. An amplitude which contains only a $g_{1}^{(1)}$ coupling represents the odd-parity $J^{P}=1^{-}$state while the an amplitude which contains only a $g_{2}^{(1)}$ coupling represents the even-parity coupling. 


\section{CHAPTER 4. KINEMATICS OF GENERIC RESONANCES}

\section{Spin-two $X$ to gauge bosons}

For a spin-two particle $X$, we write the general amplitude in terms of dependence on the field strength tensors and the polarization vectors

$$
\begin{aligned}
& A(X \rightarrow V V)=\Lambda^{-1}\left[2 g_{1}^{(2)} t_{\mu \nu} f^{* 1, \mu \alpha} f^{* 2, \nu \alpha}+2 g_{2}^{(2)} t_{\mu \nu} \frac{q_{\alpha} q_{\beta}}{\Lambda^{2}} f^{* 1, \mu \alpha} f^{* 2, \nu, \beta}\right. \\
& +g_{3}^{(2)} \frac{\tilde{q}^{\beta} \tilde{q}^{\alpha}}{\Lambda^{2}} t_{\beta \nu}\left(f^{* 1, \mu \nu} f_{\mu \alpha}^{* 2}+f^{* 2, \mu \nu} f_{\mu \alpha}^{* 1}\right)+g_{4}^{(2)} \frac{\tilde{q}^{\nu} \tilde{q}^{\mu}}{\Lambda^{2}} t_{\mu \nu} f^{* 1, \alpha \beta} f_{\alpha \beta}^{*(2)} \\
& +m_{V}^{2}\left(2 g_{5}^{(2)} t_{\mu \nu} \epsilon_{1}^{* \mu} \epsilon_{2}^{* \nu}+2 g_{6}^{(2)} \frac{\tilde{q}^{\mu} q_{\alpha}}{\Lambda^{2}} t_{\mu \nu}\left(\epsilon_{1}^{* \nu} \epsilon_{2}^{* \alpha}-\epsilon_{1}^{* \alpha} \epsilon_{2}^{* \nu}\right)+g_{7}^{(2)} \frac{\tilde{q}^{\mu} \tilde{q}^{\nu}}{\Lambda^{2}} t_{\mu \nu} \epsilon_{1}^{*} \epsilon_{2}^{*}\right) \\
& +g_{8}^{(2)} \frac{\tilde{q}_{\mu} \tilde{q}_{\nu}}{\Lambda^{2}} t_{\mu \nu} f^{* 1, \alpha \beta} \tilde{f}_{\alpha \beta}^{*(2)}+g_{9}^{(2)} t_{\mu \alpha} \tilde{q}^{\alpha} \epsilon_{\mu \nu \rho \sigma} \epsilon_{1}^{* \nu} \epsilon_{2}^{* \rho} q^{\sigma} \\
& \left.+\frac{g_{10}^{(2)} t_{\mu \alpha} \tilde{q}^{\alpha}}{\Lambda^{2}} \epsilon_{\mu \nu \rho \sigma} q^{\rho} \tilde{q}^{\sigma}\left(\epsilon_{1}^{* \nu}\left(q \epsilon_{2}^{*}\right)+\epsilon_{2}^{* \nu}\left(q \epsilon_{1}^{*}\right)\right)\right]
\end{aligned}
$$

The effective dimensionless complex coupling constants are denoted by $g_{1, \ldots, 10}^{(2)}$ where $g_{1, \ldots, 7}^{(2)}$ correspond to the parity-even interaction and $g_{8, \ldots, 10}^{(2)}$ correspond to the parity-odd interaction. While Eq. 4.4 is convenient in visualizing where each coupling contribution originates, we can rewrite it in terms of only the polarization vectors to compute the helicity amplitudes more easily. This is done in Eq. 4.5.

$$
\begin{aligned}
A(X \rightarrow Z Z)= & \Lambda^{-1} e_{1}^{* \mu} e_{2}^{* \nu}\left[c_{1}\left(q_{1} q_{2}\right) t_{\mu \nu}+c_{2} g_{\mu \nu} t_{\alpha \beta} \tilde{q}^{\alpha} \tilde{q}^{\beta}+c_{3} \frac{q_{2 \mu} q_{1 \nu}}{m_{X}^{2}} t_{\alpha \beta} \tilde{q}^{\alpha} \tilde{q}^{\beta}\right. \\
& +2 c_{4}\left(q_{1 \nu} q_{2}^{\alpha} t_{\mu \alpha}+q_{2 \mu} q_{1}^{\alpha} t_{\nu \alpha}\right)+c_{5} t_{\alpha \beta} \frac{\tilde{q}^{\alpha} \tilde{q}^{\beta}}{m_{X}^{2}} \epsilon_{\mu \nu \rho \sigma} q_{1}^{\rho} q_{2}^{\sigma} \\
& \left.+c_{6} t^{\alpha \beta} \tilde{q}_{\beta} \epsilon_{\mu \nu \alpha \rho} q^{\rho}+\frac{c_{7} t^{\alpha \beta} \tilde{q}_{\beta}}{m_{X}^{2}}\left(\epsilon_{\alpha \mu \rho \sigma} q^{\rho} \tilde{q}^{\sigma} q_{\nu}+\epsilon_{\alpha \nu \rho \sigma} q^{\rho} \tilde{q}^{\sigma} q_{\mu}\right)\right]
\end{aligned}
$$

The couplings are dependent on the dimensionless complex couplings $g_{1}^{(2)} \ldots g_{10}^{(2)}$ in the following way: 


\section{CHAPTER 4. KINEMATICS OF GENERIC RESONANCES}

$$
\begin{aligned}
& c_{1}=2 g_{1}^{(2)}+2 g_{2}^{(2)} \kappa\left(1+\frac{m_{V}^{2}}{s}\right)^{2}+2 g_{5}^{(2)} \frac{m_{V}^{2}}{s}, \\
& c_{2}=-\frac{g_{1}^{(2)}}{2}+g_{3}^{(2)} \kappa\left(1-\frac{m_{V}^{2}}{s}\right)+2 g_{4}^{(2)} \kappa+g_{7}^{(2)} \kappa \frac{m_{V}^{2}}{s}, \\
& c_{3}=-\left(\frac{g_{2}^{(2)}}{2}+g_{3}^{(2)}+2 g_{4}^{(2)}\right) \kappa \frac{m_{X}^{2}}{s}, \\
& c_{4}=-g_{1}^{(2)}-g_{2}^{(2)} \kappa-\left(g_{2}^{(2)}+g_{3}^{(2)}+g_{6}^{(2)}\right) \kappa \frac{m_{V}^{2}}{s}, \\
& c_{5}=2 g_{8}^{(2)} \kappa \frac{m_{X}^{2}}{s}, \quad c_{6}=g_{9}^{(2)}, \quad c_{7}=g_{10}^{(2)} \kappa \frac{m_{X}^{2}}{s} .
\end{aligned}
$$

The relationships in Eq. 4.6 are important when we discuss the production of such a resonance via gluon fusion. To extract the general amplitude in the case of couplings of $X$ to massless gluons, we set the mass $m_{V}=0$.

\section{Spin- $J X$ to fermions}

Finally, in discussing the production of a particle $X$ via $q \bar{q}$ annihilation and the decay of such a resonance to fermions, we introduce the general amplitude to fermions for a generic spin-zero, -one, or -two particle.

$$
\begin{gathered}
A\left(X_{J=0} \rightarrow q \bar{q}\right)=\frac{m_{q}}{v} \bar{u}_{q_{1}}\left(\rho_{1}^{(0)}+\rho_{2}^{(0)} \gamma_{5}\right) v_{q_{2}} \\
A\left(X_{J=1} \rightarrow q \bar{q}\right)=\epsilon^{\mu} \bar{u}_{q_{1}}\left(\gamma_{\mu}\left(\rho_{1}^{(1)}+\rho_{2}^{(1)} \gamma_{5}\right)+\frac{m_{q} \tilde{q}_{\mu}}{\Lambda^{2}}\left(\rho_{3}^{(1)}+\rho_{4}^{(1)} \gamma_{5}\right)\right) v_{q_{2}}, \\
A\left(X_{J=2} \rightarrow q \bar{q}\right)=\frac{1}{\Lambda} t^{\mu \nu} \bar{u}_{q_{1}}\left(\gamma_{\mu} \tilde{q}_{\nu}\left(\rho_{1}^{(2)}+\rho_{2}^{(2)} \gamma_{5}\right)+\frac{m_{q} \tilde{q}_{\mu} \tilde{q}_{\nu}}{\Lambda^{2}}\left(\rho_{3}^{(2)}+\rho_{4}^{(2)} \gamma_{5}\right)\right) v_{q_{2}},
\end{gathered}
$$

The couplings are represented by the dimensionless complex constants $\rho_{i}^{(j)}$ where $j$ is the spin of the resonance. As a reminder, the scale $\Lambda$ is often much larger than the 


\section{CHAPTER 4. KINEMATICS OF GENERIC RESONANCES}

mass of the fermions $m_{q}$ such that terms proportional to $m_{q} / \Lambda$ are suppressed. This corresponds to assumption of the chiral symmetry as a "good" quantum number. A caveat, for example, would be the decay to top quarks $t \bar{t}$.

To take an example from the SM, the couplings of a spin-one particle $X$ to fermions in Eq. 4.8 are a generalization of the weak neutral current interaction in Eq. 2.26. The structure of the interaction is similar in both equations and $\rho_{1}^{(1)}$ and $\rho_{2}^{(1)}$ are analogous to $c_{V}$ and $c_{A}$, respectively. The couplings $\rho_{1}^{(1)}$ and $\rho_{2}^{(1)}$ are often referred to as vector and axial-vector couplings to fermions. In Chapter 5, the SM interaction will be discussed in greater detail.

\subsection{Helicity amplitudes}

We are now in position to compute helicity amplitudes for the production and decay processes [33]. The helicity amplitudes are what is used to parameterize the angular distributions (see Eq. 2.15). Thus, by measuring the angular distributions in a certain process, we have access to the helicity amplitudes. By knowing how these amplitudes are expressed through effective couplings introduced in the previous section, we can constrain those couplings through measurements of angular distributions.

To compute the helicity amplitudes $A_{\lambda_{1} \lambda_{2}}$ for the decay $X \rightarrow V V$, the general amplitudes presented in the Section 4.2 are computed for a given spin configuration of the system via the polarization vectors in Eq. 4.2. In addition, the polarization vectors 


\section{CHAPTER 4. KINEMATICS OF GENERIC RESONANCES}

of the particle $X$ are required and are defined as follows. For the spin-one boson, $J_{X}=1$, we use

$$
e_{X}(0)=(0,0,0,1), \quad e_{X}( \pm)=\frac{1}{\sqrt{2}}(0, \mp 1,-i, 0) .
$$

For the spin-two boson, $J_{X}=2$, the polarization tensors read

$$
\begin{aligned}
& t^{\mu \nu}( \pm 2)=e_{X}^{\mu}( \pm) e_{X}^{\nu}( \pm), \quad t^{\mu \nu}( \pm 1)=\frac{1}{\sqrt{2}}\left[e_{X}^{\mu}( \pm) e_{X}^{\nu}(0)+e_{X}^{\mu}(0) e_{X}^{\nu}( \pm)\right] \\
& t^{\mu \nu}(0)=\frac{1}{\sqrt{6}}\left[e_{X}^{\mu}(+) e_{X}^{\nu}(-)+e_{X}^{\mu}(-) e_{X}^{\nu}(+)\right]+\sqrt{\frac{2}{3}} e_{X}^{\mu}(0) e_{X}^{\nu}(0) .
\end{aligned}
$$

For the decay to fermions, there are four possible complex amplitudes $A_{\lambda_{1} \lambda_{2}}$ where $\lambda_{1}$ and $\lambda_{2}$ are the allowed helicity states $1 / 2$ and $-1 / 2$. For the decay to two gauge bosons, there are nine complex amplitudes $A_{\lambda_{1} \lambda_{2}}$ where $\lambda_{1}$ and $\lambda_{2}$ are the allowed helicity states \pm 1 and 0 . Moreover, in the case of two identical vector bosons, such as $Z Z, g g$, or $\gamma \gamma$, the bosons are indistinguishable and the number of independent amplitudes is reduced from nine to six due to the following identity $[37,38]$

$$
A_{\lambda_{1} \lambda_{2}}=(-1)^{J} A_{\lambda_{2} \lambda_{1}},
$$

where $J$ is the spin of the $X$ particle. If parity is conserved, further constraints apply $[37,38]$

$$
A_{\lambda_{1} \lambda_{2}}=\eta_{P}(-1)^{J} A_{-\lambda_{1}-\lambda_{2}},
$$

where $\eta_{P}$ is the parity of the $X$ particle. We note that Eqs. 4.12 and 4.13 depend on phase conventions choices for the polarization vectors in Eqs. 4.2 and 4.10. 


\section{Helicity amplitudes for spin-zero decays}

Applying the polarization vectors to Eq. 4.1, the helicity amplitudes in the spin-zero case are computed to be

$$
\begin{aligned}
& A_{00}=-\frac{m_{X}^{4}}{4 v m_{V}^{2}}\left(a_{1}\left(1+\beta^{2}\right)+a_{2} \beta^{2}\right), \\
& A_{++}=\frac{m_{X}^{2}}{v}\left(a_{1}+\frac{i a_{3} \beta}{2}\right), \\
& A_{--}=\frac{m_{X}^{2}}{v}\left(a_{1}-\frac{i a_{3} \beta}{2}\right) .
\end{aligned}
$$

In the case of a decay to massless gauge bosons, the longitudinal amplitude $A_{00}$ vanishes. For the case, of the SM Higgs boson where only $a_{1} \neq 0$, the longitudinal amplitude dominates as the mass of the $X$ becomes much larger than the mass of the gauge boson $m_{V}$.

\section{Helicity amplitudes for spin-one decays}

Applying the polarization vectors to Eq. 4.3, the helicity amplitudes in the spin-one case are computed to be

$$
\begin{aligned}
& A_{+0}=-A_{0+}=\frac{\beta m_{X}^{2}}{2 m_{Z}}\left(g_{1}^{(1)}+i \beta g_{2}^{(1)}\right), \\
& A_{-0}=-A_{0-}=\frac{\beta m_{X}^{2}}{2 m_{Z}}\left(g_{1}^{(1)}-i \beta g_{2}^{(1)}\right) .
\end{aligned}
$$

It is apparent from Eq. 4.15 that a spin-one particle cannot decay to massless gauge bosons whose allowed helicity states are $\lambda= \pm 1$ since one of the gauge bosons must be longitudinally polarized $\lambda=0$. 


\section{Helicity amplitudes for spin-two decays}

Applying the polarization vectors to Eq. 4.5, the helicity amplitudes in the spin-two case are computed to be

$$
\begin{aligned}
& A_{+-}=A_{-+}=\frac{m_{X}^{2}}{4 \Lambda} c_{1}\left(1+\beta^{2}\right), \\
& A_{++}=\frac{m_{X}^{2}}{\sqrt{6} \Lambda}\left[\frac{c_{1}}{4}\left(1+\beta^{2}\right)+2 c_{2} \beta^{2}+i \beta\left(c_{5} \beta^{2}-2 c_{6}\right)\right], \\
& A_{--}=\frac{m_{X}^{2}}{\sqrt{6} \Lambda}\left[\frac{c_{1}}{4}\left(1+\beta^{2}\right)+2 c_{2} \beta^{2}-i \beta\left(c_{5} \beta^{2}-2 c_{6}\right)\right], \\
& A_{+0}=A_{0+}=\frac{m_{X}^{3}}{m_{V} \sqrt{2} \Lambda}\left[\frac{c_{1}}{8}\left(1+\beta^{2}\right)+\frac{c_{4}}{2} \beta^{2}-\frac{c_{6}+c_{7} \beta^{2}}{2} i \beta\right], \\
& A_{-0}=A_{0-}=\frac{m_{X}^{3}}{m_{V} \sqrt{2} \Lambda}\left[\frac{c_{1}}{8}\left(1+\beta^{2}\right)+\frac{c_{4}}{2} \beta^{2}+\frac{c_{6}+c_{7} \beta^{2}}{2} i \beta\right], \\
& A_{00}=\frac{m_{X}^{4}}{m_{V}^{2} \sqrt{6} \Lambda}\left[\left(1+\beta^{2}\right)\left(\frac{c_{1}}{8}-\frac{c_{2}}{2} \beta^{2}\right)-\beta^{2}\left(\frac{c_{3}}{2} \beta^{2}-c_{4}\right)\right] .
\end{aligned}
$$

It is noted that these are the helicity amplitudes in the simplified case of identical gauge bosons while in the general case, we relax the constraint from Eq. 4.12.

\section{Helicity amplitudes for decays into two fermions}

In this subsection, we write down helicity amplitudes for $X$ coupling to two fermions. Those helicity amplitudes are necessary to describe production of the resonance $X$ in $q \bar{q}$ annihilation. They are also used in the analysis of the process $q \bar{q} \rightarrow \gamma^{*} / Z \rightarrow \ell^{+} \ell^{-}$which is detailed in Chapter 5. The helicity amplitudes for the 


\section{CHAPTER 4. KINEMATICS OF GENERIC RESONANCES}

spin-zero case read

$$
\begin{aligned}
& A_{++}=\frac{m_{q}}{v} m_{X}\left(\rho_{2}^{(0)}-\beta \rho_{1}^{(0)}\right), \\
& A_{--}=\frac{m_{q}}{v} m_{X}\left(\rho_{2}^{(0)}+\beta \rho_{1}^{(0)}\right) .
\end{aligned}
$$

The helicity amplitudes for the spin-one case read

$$
\begin{aligned}
& A_{++}=-2 m_{q}\left(\rho_{1}^{(1)}+\frac{\beta m_{X}^{2}}{2 \Lambda^{2}}\left(\rho_{4}^{(1)}-\beta \rho_{3}^{(1)}\right)\right) \\
& A_{--}=-2 m_{q}\left(-\rho_{1}^{(1)}+\frac{\beta m_{X}^{2}}{2 \Lambda^{2}}\left(\rho_{4}^{(1)}+\beta \rho_{3}^{(1)}\right)\right), \\
& A_{+-}=\sqrt{2} m_{X}\left(\rho_{1}^{(1)}+\beta \rho_{2}^{(1)}\right) \\
& A_{-+}=-\sqrt{2} m_{X}\left(\rho_{1}^{(1)}-\beta \rho_{2}^{(1)}\right) .
\end{aligned}
$$

The helicity amplitudes for the spin-two case read

$$
\begin{aligned}
& A_{++}=\frac{2 \sqrt{2} m_{q} m_{X} \beta}{\sqrt{3} \Lambda}\left(\rho_{1}^{(2)}+\frac{\beta m_{X}^{2}}{2 \Lambda^{2}}\left(\rho_{4}^{(2)}-\beta \rho_{3}^{(2)}\right)\right), \\
& A_{--}=\frac{2 \sqrt{2} m_{q} m_{X} \beta}{\sqrt{3} \Lambda}\left(-\rho_{1}^{(2)}+\frac{\beta m_{X}^{2}}{2 \Lambda^{2}}\left(\rho_{4}^{(2)}+\beta \rho_{3}^{(2)}\right)\right), \\
& A_{+-}=-\frac{m_{X}^{2} \beta}{\Lambda}\left(\rho_{1}^{(2)}+\beta \rho_{2}^{(2)}\right), \\
& A_{-+}=\frac{m_{X}^{2} \beta}{\Lambda}\left(\rho_{1}^{(2)}-\beta \rho_{2}^{(2)}\right) .
\end{aligned}
$$

\subsection{Angular distributions of spin- $J$ resonances}

In the previous section, we defined the helicity amplitudes for any new particle $X$ with the SM fields. Recall in Section 2.2, Eq. 2.15, we show the relationship of the angular distributions of a given system to the helicity amplitude. This relationship is 


\section{CHAPTER 4. KINEMATICS OF GENERIC RESONANCES}

used to derive the angular distributions of a generic resonance $X$ produced at the $\mathrm{LHC}$. The angular distributions are parameterized by the helicity amplitudes derived in the previous section such that the kinematics are described for any generic resonance $X$ with spin $J$.

As discussed in Sec. 4.1, the production mechanisms which we consider in protonproton collisions are gluon fusion, $g g$, and quark-antiquark annihilation, $q \bar{q}$. We consider the decay of the particle $X$ to SM fields in two-body final states and four-body final states which is relevant for observation at the LHC. In the two-body final state, for example, we can consider the decay of $X$ to $\gamma \gamma, \ell^{+} \ell^{-}$, or $t \bar{t}$. In particular, in the next chapter, greater detail will be spent on the $\ell^{+} \ell^{-}$final state and the extraction of SM couplings. In the four-body final state, we consider $X$ decaying to $Z Z \rightarrow f_{1} \bar{f}_{1} f_{2} \bar{f}_{2}$. Specifically, the final state $Z Z \rightarrow \ell_{1}^{+} \ell_{1}^{-} \ell_{2}^{+} \ell_{2}^{-}$is known as the golden channel due to its clean reconstruction in the detector and we will investigate this in greater detail in Chapter 6 . In addition, an analysis of the $Z Z \rightarrow 2 \ell 2 q$ final state has been performed at CMS [39].

\section{Two-body Final States}

In Eq. 2.15, we show the $S$-matrix for a $2 \rightarrow 2$ process

$$
\left\langle\phi, \theta ; \lambda_{c} \lambda_{d}|S(E)| 0,0 ; \lambda_{a}, \lambda_{b}\right\rangle=\frac{1}{4 \pi} \sum_{J}(2 J+1) A_{\lambda, \mu} e^{-i(\lambda-\mu) \phi} d_{\lambda, \mu}^{j}(\theta)
$$




\section{CHAPTER 4. KINEMATICS OF GENERIC RESONANCES}

where again $\lambda=\lambda_{a}-\lambda_{b}$, and $\mu=\lambda_{c}-\lambda_{d}$ and the helicity states of the initial and final states. Let us slightly recast this expression in order to describe a $2 \rightarrow 1 \rightarrow 2$ process with an intermediate resonance produced. For a single $1 \rightarrow 2$ process, we rewrite the $S$-matrix as

$$
\left\langle\phi, \theta ; \lambda_{1} \lambda_{2}|S(E)| 0,0 ; J, m\right\rangle=\frac{1}{4 \pi} \sum_{J}(2 J+1) A_{\lambda_{1}, \lambda_{2}} e^{-i(m-\lambda) \phi} d_{m, \lambda}^{j}(\theta)
$$

Here, now we have $\lambda_{1}$ and $\lambda_{2}$ as the final state helicities and the initial state spin and projection as $|J, m\rangle$. We write the helicity amplitude as $A_{\lambda_{1}, \lambda_{2}}$ in agreement with Section 4.3 where the conservation of angular momentum fixes $m=\lambda_{1}-\lambda_{2}$.

Then using Eq. 2.17, we can write the differential cross-section for the $2 \rightarrow 1 \rightarrow 2$ process as

$$
\frac{d \Gamma\left(a b \rightarrow X_{J} \rightarrow P_{1} P_{2}\right)}{\Gamma d \cos \theta^{*}}=\left(J+\frac{1}{2}\right) \sum_{\lambda_{1}, \lambda_{2}} f_{\lambda_{1} \lambda_{2}} \sum_{\chi_{a}, \chi_{b}} f_{\chi_{a}, \chi_{b}}\left(d_{\chi_{a}-\chi_{b}, \lambda_{1}-\lambda_{2}}^{J}\left(\theta^{*}\right)\right)^{2}
$$

where we write the differential cross-section in terms of helicity fractions, $f$. These helicity fractions are related simply to the helicity amplitudes by

$$
f_{\lambda_{1}, \lambda_{2}}=\frac{\left|A_{\lambda_{1}, \lambda_{2}}\right|^{2}}{\sum_{\lambda_{1}, \lambda_{2}}\left|A_{\lambda_{1}, \lambda_{2}}\right|^{2}}
$$

and are used for convenience to parameterize the differential cross-section. Equivalent parameters have been defined for the $a b \rightarrow X_{J}$ production process as helicity states $A_{\chi_{a} \chi_{b}}$. In order to arrive at the small Wigner function present in Eq. 4.21, we have the relation $d_{\chi_{a}-\chi_{b}, \lambda_{1}-\lambda_{2}}^{J}\left(\theta^{*}\right)=d_{\chi_{a}-\chi_{b}, m}^{J}\left(\theta_{0}\right) \times d_{m, \lambda_{1}-\lambda_{2}}^{J}\left(\theta^{*}\right)$. Then, the reference system is defined is for convenience by setting $\theta_{0}=0$ and conservation of momentum is enforced 


\section{CHAPTER 4. KINEMATICS OF GENERIC RESONANCES}

where $\chi_{a}-\chi_{b}=m=\lambda_{1}-\lambda_{2}$. The result in Eq. 4.21 is the general expression for the angular distribution of a $2 \rightarrow 2$ process at the LHC.

\section{Example of a $2 \rightarrow 2$ process: $X \rightarrow \gamma \gamma$}

For the decay of a particle $X$ with spin $J$ to two photons, only the $J=2$ case is considered. For a $J=0$ particle, the particle $X$ holds no spin information and the angular distribution is trivially uniform. Decay of a $J=1$ particle to two massless photons is forbidden due to the Landau-Yang theorem. For the $J=2$ case, we start from Eq. 4.21. Note that for $f_{\lambda_{1} \lambda_{2}}$ only the $f_{++}, f_{--}$and $f_{-+}=f_{+-}$parameters are non-zero in the decay to two massless identical photons.

In the case of the production helicity fractions $f_{\chi_{1} \chi_{2}}$, it is more convenient to write three real parameters $f_{z 0}, f_{z 1}$, and $f_{z 2}$ to describe the fraction of spin-two $X$ resonance production with spin $z$-projections $0, \pm 1$, and \pm 2 , respectively. The fractions $f_{z 0}$ or $f_{z 2}$ arise from the gluon fusion mechanism with amplitudes $A_{++}$and $A_{--}$or $A_{+-}$and $A_{-+}$in Eq. 4.16, respectively. The fraction $f_{z 1}$ originates from $q \bar{q}$ annihilation with amplitudes $B_{+-}$and $B_{-+}$in Eq. 4.19. Note that because we assume that initial state quarks are massless, only \pm 1 spin projections on the $z$ axis are possible in quarkanitquark annihilation. On the other hand, both $J_{z}= \pm 2$ and $J_{z}=0$ spin projections of a spin-two $X$ are possible in gluon fusion. The latter possibility is often ignored in the literature because, accidentally, this contribution vanishes in the case of the minimal coupling of the spin-two particle to massless gauge bosons. 


\section{CHAPTER 4. KINEMATICS OF GENERIC RESONANCES}

We can then write down the angular distribution for a spin-two $X$ decaying to two photons as

$$
\begin{aligned}
\frac{16 d \Gamma\left(X_{J=2} \rightarrow \gamma \gamma\right)}{5 \Gamma d \cos \theta^{*}}= & \left(2-2 f_{z 1}+f_{z 2}\right)-6\left(2-4 f_{z 1}-f_{z 2}\right) \cos ^{2} \theta^{*}+3\left(6-10 f_{z 1}-5 f_{z 2}\right) \cos ^{4} \theta^{*} \\
+ & f_{+-}\left[\left(2+2 f_{z 1}-7 f_{z 2}\right)+6\left(2-6 f_{z 1}+f_{z 2}\right) \cos ^{2} \theta^{*}\right. \\
& \left.-5\left(6-10 f_{z 1}-5 f_{z 2}\right) \cos ^{4} \theta^{*}\right] .
\end{aligned}
$$

\section{Example of a $2 \rightarrow 2$ process: $X \rightarrow f \bar{f}$}

For the decay of a particle $X$ to fermions, we consider the spin-one and spin-two cases. In the $J=1$ case, recall that the only production mechanism is $q \bar{q}$. The helicity amplitudes are taken from Eq. 4.18 where the terms dependent on $\rho_{3}^{(1)}$ and $\rho_{4}^{(1)}$ are suppressed under the assumption of light fermions. The angular distribution is

$$
\begin{aligned}
\frac{8 d \Gamma\left(X_{J=1} \rightarrow f \bar{f}\right)}{3 \Gamma d \cos \theta^{*}}= & \left(f_{+-}+f_{-+}\right)\left(1+\cos ^{2} \theta^{*}\right)+2\left(1-f_{+-}-f_{-+}\right)\left(1-\cos ^{2} \theta^{*}\right) \\
& +2\left(f_{+-}-f_{-+}\right) \Delta f_{z 1} \cos \theta^{*}
\end{aligned}
$$

We define $\Delta f_{z 1}$ as the difference between the fraction of +1 and -1 spin projections due to the production mechanism. It follows from this formula that there is a forwardbackward asymmetry in this decay, as was pointed out in Ref. [40] in the context of spin-one decays to a fermion pair. A dilution factor needs to be introduced in front of the $\Delta f_{z 1}$ terms, which depends on the ability to measure the sign of $\cos \theta^{*}$ in an experiment. This case is explored in greater detail when presenting the measurement of $\sin ^{2} \theta_{W}$ in Chapter 5 . 


\section{CHAPTER 4. KINEMATICS OF GENERIC RESONANCES}

For the case of $J=2$, we use a similar notation but production is allowed via gluon fusion and quark-antiquark annihilation. The possible spin projections of the resonance are $0, \pm 1, \pm 2$ and the angular distribution is given as

$$
\begin{aligned}
\frac{16 d \Gamma\left(X_{J=2} \rightarrow f \bar{f}\right)}{5 \Gamma d \cos \theta^{*}}= & 2\left(f_{+-}+f_{-+}\right)\left\{\left(f_{z 1}+f_{z 2}\right)+3\left(2-3 f_{z 1}-2 f_{z 2}\right) \cos ^{2} \theta^{*}\right. \\
& \left.-\left(6-10 f_{z 1}-5 f_{z 2}\right) \cos ^{4} \theta^{*}\right\} \\
& +\left(1-f_{+-}-f_{-+}\right)\left\{\left(2-2 f_{z 1}+f_{z 2}\right)-6\left(2-4 f_{z 1}-f_{z 2}\right) \cos ^{2} \theta^{*}\right. \\
& \left.+3\left(6-10 f_{z 1}-5 f_{z 2}\right) \cos ^{4} \theta^{*}\right\} \\
& -4\left(f_{+-}-f_{-+}\right) \Delta f_{z 1}\left(\cos \theta^{*}-2 \cos ^{3} \theta^{*}\right) .
\end{aligned}
$$

\section{Four-body Final States}

We now turn to the case of a resonance $X$ with spin $J$ decaying in a four-body final state of the process type $2 \rightarrow 4$. In particular, we focus on the process $p p \rightarrow X \rightarrow$ $Z_{1} Z_{2} \rightarrow\left(f_{1} \bar{f}_{1}\right)\left(f_{2} \bar{f}_{2}\right)$ where the four final state fermions can be reconstructed fully. More information is contained in the four-body final state which gives it an advantage over the two-body case. In the $2 \rightarrow 2$ process, there is only one non-trivial angle $\cos \theta^{*}$ and degeneracies between various signal hypotheses are more likely to occur. In general, the $2 \rightarrow 4$ process contains 5 non-trivial angles which are illustrated in Fig. 4.4. The exact definition of the five angles shown in Fig. 4.4 is given subsequently. The angle $\theta^{*}$ is the polar angle between the parton collision axis $z$ and the $X \rightarrow Z_{1} Z_{2}$ decay axis $z^{\prime}$, both defined in the $X$ rest frame. The angle $\Psi$ is the azimuthal angle 


\section{CHAPTER 4. KINEMATICS OF GENERIC RESONANCES}

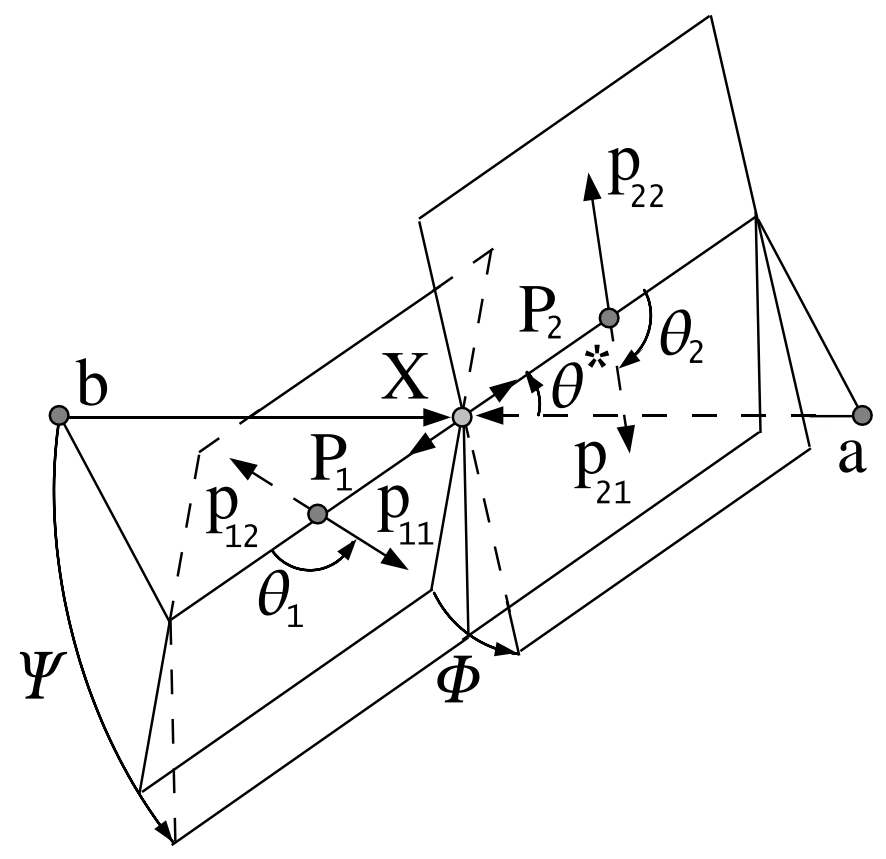

Figure 4.4: Angular distribution for $2 \rightarrow 4$ process.

between the $z z^{\prime}$ plane and the plane of which bisects the $Z_{1} \rightarrow\left(f_{1} \bar{f}_{1}\right)$ and $Z_{2} \rightarrow\left(f_{2} \bar{f}_{2}\right)$ decay planes in the $X$ rest frame. The angles $\theta^{*}$ and $\Psi$ are denoted as production angles because they depend on the production mechanism, $g g$ or $q \bar{q}$. The angle $\Psi$ could also be defined to the $Z_{1}$ or $Z_{2}$ decay plane (defined at $\Phi_{1}$ and $\Phi_{2}$, respectively) but is defined as above for the simplicity of the algebraic expression given in Eq. 4.34. The angle $\Phi$ is the angle between the decay planes of the two $Z$ systems in the $X$ rest frame. The relationship between the azimuthal angles is $\Psi=\Phi_{1}+\Phi / 2$. The angles $\Psi$ and $\Phi_{1}$ are used interchangeably below to denote the azimuthal production angle and are related by the simple expression above. The angle $\theta_{i}$ is the angle between the direction of the fermion $f_{i}$ from $Z_{i} \rightarrow\left(f_{i} \bar{f}_{i}\right)$ and the direction opposite the $X$ in 


\section{CHAPTER 4. KINEMATICS OF GENERIC RESONANCES}

the $Z_{i}$ rest frame, where index $i=1,2$ refers to the first or second $Z$ boson. Finally, The angles $\Phi, \theta_{1}$ and $\theta_{2}$ do not depend on the production mechanism and are denoted as the helicity angles.

The sixth angle $\Phi^{*}$ is the azimuth angle of the $z^{\prime}$ axis with respect to $z$. It can be arbitrarily defined and it does not carry any information about the process. However, $\Phi^{*}$ can be used for bookkeeping purposes to differentiate between the first and second $Z$ in the decay in the case that both have identical decay channels.

To derive the angular distribution, we again start with the description of the $1 \rightarrow 2$ process given in Eq. 4.20. If we now envision the process $a b \rightarrow X \rightarrow V_{1} V_{2} \rightarrow$ $\left(f_{1} f_{1}^{\prime}\right)\left(f_{2} f_{2}^{\prime}\right)$ as a sequence of $1 \rightarrow 2$ processes, it can be described as

$$
\begin{aligned}
& A_{a b}\left(p_{a}, p_{b} ; \chi_{1}, \chi_{2}, m, \lambda_{1}, \lambda_{2}, \mu_{1}, \mu_{2}, \tau_{1}, \tau_{2} ; \Omega^{*}, \Omega, \Omega^{\prime}, \Omega^{\prime \prime}\right)=\frac{(2 J+1)}{4 \pi} \sqrt{\frac{\left(2 s_{1}+1\right)}{4 \pi} \frac{\left(2 s_{2}+1\right)}{4 \pi}} \\
& \times D_{\chi_{1}-\chi_{2}, m}^{J *}\left(\Omega^{*}\right) B_{\chi_{1} \chi_{2}} \times D_{m, \lambda_{1}-\lambda_{2}}^{J *}(\Omega) A_{\lambda_{1} \lambda_{2}} \times D_{\lambda_{1}, \mu_{1}-\mu_{2}}^{s_{1} *}\left(\Omega^{\prime}\right) T\left(\mu_{1}, \mu_{2}\right) \times D_{\lambda_{2}, \tau_{1}-\tau_{2}}^{s_{2} *}\left(\Omega^{\prime \prime}\right) W\left(\tau_{1}, \tau_{2}\right) .
\end{aligned}
$$

Here the $A_{\lambda_{1} \lambda_{2}}$ and $B_{\chi_{1} \chi_{2}}$ amplitudes correspond to the $X$ decay and production processes, respectively; $T\left(\mu_{1}, \mu_{2}\right)$ and $W\left(\tau_{1}, \tau_{2}\right)$ describe the decays of the first and the second $X$ daughter to pairs of fermions; $\lambda_{1,2}$ are the helicities of the two $X$ daughters; $\chi_{1,2}$ are the helicities of two gluons or quarks in the initial state; $\mu_{1,2}$ and $\tau_{1,2}$ are the helicities of fermions in the $Z$ decays; and $m$ denotes the helicity of $X$. We keep the notation general enough with $J$ denoting the spin of the $X$ boson and $s_{1,2}$ referring to the spins of its decay products, e.g. the $Z$ bosons. In particular, $T\left(\mu_{1}, \mu_{2}\right)$ and $W\left(\tau_{1}, \tau_{2}\right)$ is specific to the decay of $Z$ bosons and is explored in great detail in Chapter 5 . We choose the convention where $\Omega=(0,0,0)$, which by conservation of 


\section{CHAPTER 4. KINEMATICS OF GENERIC RESONANCES}

angular momentum effectively sets $m=\lambda_{1}-\lambda_{2}, \Omega^{*}=\left(\Phi_{1}, \theta^{*},-\Phi_{1}\right), \Omega^{\prime}=\left(0, \theta_{1}, 0\right)$ and $\Omega^{\prime \prime}=\left(\Phi, \theta_{2},-\Phi\right)$.

Summing over the helicities of all particles, we obtain the differential cross-section for the process $a b \rightarrow X \rightarrow V_{1} V_{2} \rightarrow\left(f_{1} f_{1}^{\prime}\right)\left(f_{2} f_{2}^{\prime}\right)$ as

$$
\begin{aligned}
& \frac{d \sigma_{a b}\left(p_{a}, p_{b}, \theta^{*}, \Phi_{1}, \theta_{1}, \theta_{2}, \Phi\right)}{\mathrm{d} Y_{X} d \cos \theta^{*} d \Phi_{1} d \cos \theta_{1} d \cos \theta_{2} d \Phi}= \\
& \quad \mathcal{N}_{a b} \delta\left(s_{a b}-m_{X}^{2}\right) \delta\left(Y_{a b}-Y_{X}\right) \sum_{\{\chi, \mu, \tau\}}\left|\sum_{\{\lambda, m\}} A_{a b}\left(p_{a}, p_{b} ;\{\chi, \lambda ; m, \mu, \tau\} ;\{\Omega\}\right)\right|^{2}
\end{aligned}
$$

where $s_{a b}$ is the partonic center-of-mass energy squared, $Y_{a b}$ and $Y_{X}$ are the rapidities of the colliding partons $a b$ and the resonance $X$, and $\mathcal{N}_{a b}$ is the normalization factor

$$
\mathcal{N}_{a b}=\frac{\pi \beta_{Z}}{8 m_{X}^{3} \Gamma_{X} c_{a b}\left(32 \pi^{2}\right)^{3}\left(2 m_{Z} \Gamma_{Z}\right)^{2}},
$$

with $\beta_{Z}=\sqrt{1-4 m_{Z}^{2} / m_{X}^{2}}, c_{q \bar{q}}=3, c_{g g}=8$, and $\Gamma_{X, Z}$ being the decay widths of the resonance $X$ and the $Z$ boson, respectively. The relevant differential cross-section for hadron collisions is obtained by convoluting parton cross-sections with parton distribution functions

$$
\begin{aligned}
& \frac{d \sigma_{p p}\left(\theta^{*}, \Phi_{1}, \theta_{1}, \theta_{2}, \Phi\right)}{d \cos \theta^{*} d \Phi_{1} d \cos \theta_{1} d \cos \theta_{2} d \Phi}= \\
& \left.\quad \sum_{a b} \int \mathrm{d} Y_{X} \mathrm{~d} x_{1} \mathrm{~d} x_{2} \tilde{f}_{a}\left(x_{1}\right) \tilde{f}_{b}\left(x_{2}\right) \frac{d \sigma_{a b}\left(x_{1} p_{1}, x_{2} p_{2}, \theta^{*}, \Phi_{1}, \theta_{1}, \theta_{2}, \Phi\right)}{\mathrm{d} Y_{X} d \cos \theta^{*} d \Phi_{1} d \cos \theta_{1} d \cos \theta_{2} d \Phi}\right|_{Y_{a b}=\frac{1}{2} \ln \frac{x_{1}}{x_{2}}}
\end{aligned}
$$

where $p_{1,2}$ are the momenta of the two protons. The inclusion of the effect of the parton distribution functions in the differential cross-section is reviewed in greater detail in Section 5.1 when discussing the measurement of the weak mixing angle. Here, we only point out that the momentum of the parton constituents within the 


\section{CHAPTER 4. KINEMATICS OF GENERIC RESONANCES}

proton are described by these parton distribution functions and must be included in the calculation of the differential cross-section. However in the current discussion of the angular distributions, we note that the angles $\cos \theta^{*}, \Phi_{1}, \cos \theta_{1}, \cos \theta_{2}, \Phi$ are defined in the $X$ rest frame and are not affected by the integrations over $x_{1}, x_{2}$, and $Y_{X}$. Convolution with the parton distribution functions results in the rapidity distribution of the $X$ boson and may affect angular distributions of decay products on an event-byevent basis due to the detector acceptance, discussed later. However, it does not affect angular distributions measured with the ideal detector since in this case, integrations over $x_{1}, x_{2}$, and $Y_{X}$ in Eq. (4.30) factorize from all angular dependences. As the result, we may rewrite Eq. (4.30) in the form of the angular distribution of the decay products of the polarized particle $X$

$$
\frac{1}{\sigma_{p p \text { tot }}} \frac{d \sigma_{p p}}{d \cos \theta^{*} d \Phi_{1} d \cos \theta_{1} d \cos \theta_{2} d \Phi}=\frac{1}{\Gamma_{X}} \frac{d \Gamma_{X}}{d \cos \theta^{*} d \Phi_{1} d \cos \theta_{1} d \cos \theta_{2} d \Phi} .
$$

Since we are interested in the normalized distributions, many normalization factors, as in Eq. 4.28, drop out.

Similar to how the parameters are defined in the $2 \rightarrow 2$ case, we define easyto-measure parameters related to the helicity amplitudes, The case of a spin-two resonance is considered as an example. The decay of a spin-two particle to two vector bosons is characterized by nine complex helicity amplitudes $A_{\lambda_{1} \lambda_{2}}$. Six complex amplitudes are parameterized by twelve real numbers. However, since we are interested in the normalized angular distribution, two of them, the normalization and the overall phase, are unobservable. Hence, angular distributions of the spin-two particle decay 


\section{CHAPTER 4. KINEMATICS OF GENERIC RESONANCES}

to two identical vector bosons can be parameterized by ten real parameters. We can choose them to be

$$
\begin{aligned}
f_{\lambda_{1} \lambda_{2}} & =\left|A_{\lambda_{1} \lambda_{2}}\right|^{2} / \sum_{k, l= \pm, 0}\left|A_{k l}\right|^{2}, \\
\phi_{\lambda_{1} \lambda_{2}} & =\arg \left(A_{\lambda_{1} \lambda_{2}} / A_{00}\right),
\end{aligned}
$$

with $\left(\lambda_{1}, \lambda_{2}\right)=(++),(--),(+-),(+0),(0-)$. The remaining (dependent) amplitude parameters $f_{k l}$ and $\phi_{k l}$ can be expressed either using Eq. 4.12 or the relationship

$$
f_{00}=1-\sum_{k, l= \pm, 0} f_{k l}, \quad \phi_{00}=0 .
$$

For the $X$ decays to massless vector bosons or if its spin is less than two, the number of independent non-vanishing parameters is further reduced. Our notation for those (simpler) cases are similar to what is described above. For example, the spin-zero case is parameterized with four real parameters for $\left(\lambda_{1}, \lambda_{2}\right)=(++),(--)$, and the spin-one case with two real parameters for $\left(\lambda_{1}, \lambda_{2}\right)=(+0)$ after re-defining the phase convention with respect to the $A_{0-}$ amplitude.

Again, in the case of the production helicity states, we define them as fractions of the resonance spin-projections. Exactly as in the case of the $2 \rightarrow 2$ process, we choose the three real parameters $f_{z 0}, f_{z 1}$, and $f_{z 2}$ to describe the fraction of spin-two $X$ resonance production.

Equation 4.27 can now be expanded as a full sum over all possible helicities for a generic spin $J$ particle to calculate the full angular distribution. The full expression is given and further discussion of the implications is discussed below.

$$
\frac{\mathcal{N}_{J} d \Gamma_{J}}{\Gamma d \cos \theta^{*} d \Psi d \cos \theta_{1} d \cos \theta_{2} d \Phi}=
$$




\section{CHAPTER 4. KINEMATICS OF GENERIC RESONANCES}

$$
\begin{aligned}
& F_{00}^{J}\left(\theta^{*}\right) \times\left\{4 f_{00} \sin ^{2} \theta_{1} \sin ^{2} \theta_{2}+\left(f_{++}+f_{--}\right)\left(\left(1+\cos ^{2} \theta_{1}\right)\left(1+\cos ^{2} \theta_{2}\right)+4 R_{1} R_{2} \cos \theta_{1} \cos \theta_{2}\right)\right. \\
& -2\left(f_{++}-f_{--}\right)\left(R_{1} \cos \theta_{1}\left(1+\cos ^{2} \theta_{2}\right)+R_{2}\left(1+\cos ^{2} \theta_{1}\right) \cos \theta_{2}\right) \\
& +4 \sqrt{f_{++} f_{00}}\left(R_{1}-\cos \theta_{1}\right) \sin \theta_{1}\left(R_{2}-\cos \theta_{2}\right) \sin \theta_{2} \cos \left(\Phi+\phi_{++}\right) \\
& +4 \sqrt{f_{--} f_{00}}\left(R_{1}+\cos \theta_{1}\right) \sin \theta_{1}\left(R_{2}+\cos \theta_{2}\right) \sin \theta_{2} \cos \left(\Phi-\phi_{--}\right) \\
& \left.+2 \sqrt{f_{++} f_{--}} \sin ^{2} \theta_{1} \sin ^{2} \theta_{2} \cos \left(2 \Phi+\phi_{++}-\phi_{--}\right)\right\} \\
& +4 F_{11}^{J}\left(\theta^{*}\right) \times\left\{\left(f_{+0}+f_{0-}\right)\left(1-\cos ^{2} \theta_{1} \cos ^{2} \theta_{2}\right)-\left(f_{+0}-f_{0-}\right)\left(R_{1} \cos \theta_{1} \sin ^{2} \theta_{2}+R_{2} \sin ^{2} \theta_{1} \cos \theta_{2}\right)\right. \\
& \left.+2 \sqrt{f_{+0} f_{0-}} \sin \theta_{1} \sin \theta_{2}\left(R_{1} R_{2}-\cos \theta_{1} \cos \theta_{2}\right) \cos \left(\Phi+\phi_{+0}-\phi_{0-}\right)\right\} \\
& +(-1)^{J} \times 4 F_{-11}^{J}\left(\theta^{*}\right) \times\left\{\left(f_{+0}+f_{0-}\right)\left(R_{1} R_{2}+\cos \theta_{1} \cos \theta_{2}\right)-\left(f_{+0}-f_{0-}\right)\left(R_{1} \cos \theta_{2}+R_{2} \cos \theta_{1}\right)\right. \\
& \left.+2 \sqrt{f_{+0} f_{0-}} \sin \theta_{1} \sin \theta_{2} \cos \left(\Phi+\phi_{+0}-\phi_{0-}\right)\right\} \sin \theta_{1} \sin \theta_{2} \cos (2 \Psi) \\
& +2 F_{22}^{J}\left(\theta^{*}\right) \times f_{+-}\left\{\left(1+\cos ^{2} \theta_{1}\right)\left(1+\cos ^{2} \theta_{2}\right)-4 R_{1} R_{2} \cos \theta_{1} \cos \theta_{2}\right\} \\
& +(-1)^{J} \times 2 F_{-22}^{J}\left(\theta^{*}\right) \times f_{+-} \sin ^{2} \theta_{1} \sin ^{2} \theta_{2} \cos (4 \Psi) \\
& +2 F_{02}^{J}\left(\theta^{*}\right) \times\left\{2 \sqrt{f_{00} f_{+-}} \sin \theta_{1} \sin \theta_{2} \times\left[\left(R_{1}-\cos \theta_{1}\right)\left(R_{2}+\cos \theta_{2}\right) \cos \left(2 \Psi-\phi_{+-}\right)\right.\right. \\
& \left.+\left(R_{1}+\cos \theta_{1}\right)\left(R_{2}-\cos \theta_{2}\right) \cos \left(2 \Psi+\phi_{+-}\right)\right] \\
& +\sqrt{f_{++} f_{+-}}\left[\sin ^{2} \theta_{1}\left(1-2 R_{2} \cos \theta_{2}+\cos ^{2} \theta_{2}\right) \cos \left(2 \Psi-\Phi+\phi_{+-}-\phi_{++}\right)\right. \\
& \left.+\left(1-2 R_{1} \cos \theta_{1}+\cos ^{2} \theta_{1}\right) \sin ^{2} \theta_{2} \cos \left(2 \Psi+\Phi-\phi_{+-}+\phi_{++}\right)\right] \\
& +\sqrt{f_{--} f_{+-}}\left[\sin ^{2} \theta_{1}\left(1+2 R_{2} \cos \theta_{2}+\cos ^{2} \theta_{2}\right) \cos \left(2 \Psi-\Phi-\phi_{+-}+\phi_{--}\right)\right. \\
& \left.\left.+\left(1+2 R_{1} \cos \theta_{1}+\cos ^{2} \theta_{1}\right) \sin ^{2} \theta_{2} \cos \left(2 \Psi+\Phi+\phi_{+-}-\phi_{--}\right)\right]\right\} \\
& -2 \sqrt{2} F_{01}^{J}\left(\theta^{*}\right) \times\left\{2 \sqrt { f _ { 0 0 } f _ { + 0 } } \left[\sin \theta_{1}\left(R_{1}-\cos \theta_{1}\right) \sin ^{2} \theta_{2} \cos \left(\Psi-\Phi / 2-\phi_{+0}\right)\right.\right. \\
& \left.-\sin ^{2} \theta_{1} \sin \theta_{2}\left(R_{2}-\cos \theta_{2}\right) \cos \left(\Psi+\Phi / 2+\phi_{+0}\right)\right] \\
& +2 \sqrt{f_{00} f_{0-}}\left[\sin ^{2} \theta_{1} \sin \theta_{2}\left(R_{2}+\cos \theta_{2}\right) \cos \left(\Psi+\Phi / 2-\phi_{0-}\right)\right. \\
& \left.-\sin \theta_{1}\left(R_{1}+\cos \theta_{1}\right) \sin ^{2} \theta_{2} \cos \left(\Psi-\Phi / 2+\phi_{0-}\right)\right] \\
& +\sqrt{f_{++} f_{+0}}\left[\left(1-2 R_{1} \cos \theta_{1}+\cos ^{2} \theta_{1}\right) \sin \theta_{2}\left(R_{2}-\cos \theta_{2}\right) \cos \left(\Psi+\Phi / 2+\phi_{++}-\phi_{+0}\right)\right.
\end{aligned}
$$




\section{CHAPTER 4. KINEMATICS OF GENERIC RESONANCES}

$$
\begin{aligned}
& \left.-\sin \theta_{1}\left(R_{1}-\cos \theta_{1}\right)\left(1-2 R_{2} \cos \theta_{2}+\cos ^{2} \theta_{2}\right) \cos \left(\Psi-\Phi / 2-\phi_{++}+\phi_{+0}\right)\right] \\
& +\sqrt{f_{++} f_{0-}}\left[\sin \theta_{1}\left(R_{1}-\cos \theta_{1}\right) \sin ^{2} \theta_{2} \cos \left(\Psi+3 \Phi / 2+\phi_{++}-\phi_{0-}\right)\right. \\
& \left.-\sin ^{2} \theta_{1} \sin \theta_{2}\left(R_{2}-\cos \theta_{2}\right) \cos \left(\Psi-3 \Phi / 2-\phi_{++}+\phi_{0-}\right)\right] \\
& +\sqrt{f_{--} f_{+0}}\left[\sin ^{2} \theta_{1} \sin \theta_{2}\left(R_{2}+\cos \theta_{2}\right) \cos \left(\Psi-3 \Phi / 2+\phi_{--}-\phi_{+0}\right)\right. \\
& \left.-\sin \theta_{1}\left(R_{1}+\cos \theta_{1}\right) \sin ^{2} \theta_{2} \cos \left(\Psi+3 \Phi / 2-\phi_{--}+\phi_{+0}\right)\right] \\
& +\sqrt{f_{--} f_{0-}}\left[\sin \theta_{1}\left(R_{1}+\cos \theta_{1}\right)\left(1+2 R_{2} \cos \theta_{2}+\cos ^{2} \theta_{2}\right) \cos \left(\Psi-\Phi / 2+\phi_{--}-\phi_{0-}\right)\right. \\
& \left.\left.-\left(1+2 R_{1} \cos \theta_{1}+\cos ^{2} \theta_{1}\right) \sin \theta_{2}\left(R_{2}+\cos \theta_{2}\right) \cos \left(\Psi+\Phi / 2-\phi_{--}+\phi_{0-}\right)\right]\right\} \\
& -2 \sqrt{2} F_{12}^{J}\left(\theta^{*}\right) \times\left\{\sqrt { f _ { + - } f _ { + 0 } } \left[\left(1-2 R_{1} \cos \theta_{1}+\cos ^{2} \theta_{1}\right) \sin \theta_{2}\left(R_{2}+\cos \theta_{2}\right) \cos \left(\Psi+\Phi / 2-\phi_{+-}+\phi_{+0}\right)\right.\right. \\
& \left.-\sin \theta_{1}\left(R_{1}+\cos \theta_{1}\right)\left(1-2 R_{2} \cos \theta_{2}+\cos ^{2} \theta_{2}\right) \cos \left(\Psi-\Phi / 2+\phi_{+-}-\phi_{+0}\right)\right] \\
& +\sqrt{f_{+-} f_{0-}}\left[\sin \theta_{1}\left(R_{1}-\cos \theta_{1}\right)\left(1+2 R_{2} \cos \theta_{2}+\cos ^{2} \theta_{2}\right) \cos \left(\Psi-\Phi / 2-\phi_{+-}+\phi_{0-}\right)\right. \\
& \left.\left.-\left(1+2 R_{1} \cos \theta_{1}+\cos ^{2} \theta_{1}\right) \sin \theta_{2}\left(R_{2}-\cos \theta_{2}\right) \cos \left(\Psi+\Phi / 2+\phi_{+-}-\phi_{0-}\right)\right]\right\} \\
& -(-1)^{J} \times 2 \sqrt{2} F_{-12}^{J}\left(\theta^{*}\right) \times\left\{\sqrt { f _ { + - } f _ { + 0 } } \left[\sin \theta_{1}\left(R_{1}-\cos \theta_{1}\right) \sin ^{2} \theta_{2} \cos \left(3 \Psi+\Phi / 2-\phi_{+-}+\phi_{+0}\right)\right.\right. \\
& \left.-\sin ^{2} \theta_{1} \sin \theta_{2}\left(R_{2}-\cos \theta_{2}\right) \cos \left(3 \Psi-\Phi / 2+\phi_{+-}-\phi_{+0}\right)\right] \\
& +\sqrt{f_{+-} f_{0-}}\left[\sin ^{2} \theta_{1} \sin \theta_{2}\left(R_{2}+\cos \theta_{2}\right) \cos \left(3 \Psi-\Phi / 2-\phi_{+-}+\phi_{0-}\right)\right. \\
& \left.\left.-\sin \theta_{1}\left(R_{1}+\cos \theta_{1}\right) \sin ^{2} \theta_{2} \cos \left(3 \Psi+\Phi / 2+\phi_{+-}-\phi_{0-}\right)\right]\right\},
\end{aligned}
$$

where $\mathcal{N}_{J}$ is the normalization factor which does not affect the angular distributions.

The angular distribution above is parameterized by the helicity fractions and phases, $f_{\lambda_{1} \lambda_{2}}$ and $\phi_{\lambda_{1} \lambda_{2}}$ which are determined for a given signal scenario. Due to the SM $Z$ decays, distributions depend on the parameters $R_{1,2}=2 r_{1,2} /\left(1+r_{1,2}^{2}\right)$, where $r_{1,2}$ is the ratio of axial to vector couplings of the fermions $f_{1,2}$. Specifically, $r=c_{A} / c_{V}=$ $t_{3 L} /\left(t_{3 L}-2 q \sin ^{2} \theta_{W}\right)$, where $q$ is the fermion charge and $t_{3 L}$ is its weak isospin, as

discussed in Section 2.3. This dependence of $Z$ decays on $\sin ^{2} \theta_{W}$ is the central 


\section{CHAPTER 4. KINEMATICS OF GENERIC RESONANCES}

concept to be discussed in Chapter 5 and more details are given there. If we consider $Z$ decays to charged leptons, $q=-1, t_{3 L}=-1 / 2$, and $R \simeq 0.15$. Note that if the first $Z$ decays to charged leptons and the second $Z$ decays to quark and antiquark jets, one needs to average the value of $R_{2}$ over contributing quark flavors since $R$ is different for up and down quarks, $R_{\text {up }} \simeq 0.67, R_{\text {down }} \simeq 0.94$. However, if the quark and antiquark jets cannot be distinguished in the $Z$ decay, this is equivalent to $R_{2}=0$ in the measured angular distributions.

The functions $F_{i j}^{J}\left(\theta^{*}\right)$ are explicitly dependent on the polar production angle $\theta^{*}$. They are defined as follows

$$
F_{i j}^{J}\left(\theta^{*}\right)=\sum_{m=0, \pm 1, \pm 2} f_{m} d_{m i}^{J}\left(\theta^{*}\right) d_{m j}^{J}\left(\theta^{*}\right)
$$

where $f_{ \pm 1}=f_{z 1} / 2, f_{ \pm 2}=f_{z 2} / 2$, and $f_{0}=f_{z 0}=\left(1-f_{z 1}-f_{z 2}\right)$. Note that for odd $J$ one has $f_{00}=f_{++}=f_{--}=0$, and therefore $F_{0 j}^{J=\text { odd }}\left(\theta^{*}\right)$ terms do not contribute.

Below we show $F_{i j}^{J}\left(\theta^{*}\right)$ explicitly for $J=0,1$, and 2 . For spin-zero, we have

$$
\begin{aligned}
& F_{00}^{0}=1, \\
& F_{11}^{0}=F_{-11}^{0}=F_{22}^{0}=F_{-22}^{0}=F_{02}^{0}=F_{01}^{0}=F_{12}^{0}=F_{-12}^{0}=0,
\end{aligned}
$$

where only four parameters $f_{++}, f_{--}, \phi_{++}$, and $\phi_{--}$remain relevant, and $f_{00}$ can be expressed as $f_{00}=\left(1-f_{++}-f_{--}\right)$. For spin-one, we have

$$
\begin{aligned}
& F_{11}^{1}=\frac{1}{4}\left(1+\cos ^{2} \theta^{*}\right), \\
& F_{-11}^{1}=\frac{1}{4}\left(1-\cos ^{2} \theta^{*}\right), \\
& F_{00}^{1}=F_{22}^{1}=F_{-22}^{1}=F_{02}^{1}=F_{01}^{1}=F_{12}^{1}=F_{-12}^{1}=0,
\end{aligned}
$$




\section{CHAPTER 4. KINEMATICS OF GENERIC RESONANCES}

where only two parameters $f_{+0}$ and $\left[\phi_{+0}-\phi_{0-}\right]$ remain relevant, and $f_{0-}$ can be expressed as $f_{0-}=\left(1-2 f_{+0}\right) / 2$. Finally, for spin-two, we have

$$
\begin{aligned}
& F_{00}^{2}=\frac{1}{8}\left\{\left(2-2 f_{z 1}+f_{z 2}\right)-6\left(2-4 f_{z 1}-f_{z 2}\right) \cos ^{2} \theta^{*}+3\left(6-10 f_{z 1}-5 f_{z 2}\right) \cos ^{4} \theta^{*}\right\} \\
& F_{11}^{2}=\frac{1}{4}\left\{\left(f_{z 1}+f_{z 2}\right)+3\left(2-3 f_{z 1}-2 f_{z 2}\right) \cos ^{2} \theta^{*}-\left(6-10 f_{z 1}-5 f_{z 2}\right) \cos ^{4} \theta^{*}\right\} \\
& F_{-11}^{2}=-\frac{1}{4}\left\{\left(f_{z 1}-f_{z 2}\right)+\left(6-10 f_{z 1}-5 f_{z 2}\right) \cos ^{2} \theta^{*}\right\} \sin ^{2} \theta^{*} \\
& F_{22}^{2}=\frac{1}{16}\left\{\left(6-2 f_{z 1}-5 f_{z 2}\right)-6\left(2-2 f_{z 1}-3 f_{z 2}\right) \cos ^{2} \theta^{*}+\left(6-10 f_{z 1}-5 f_{z 2}\right) \cos ^{4} \theta^{*}\right\} \\
& F_{-22}^{2}=\frac{1}{16}\left\{6-10 f_{z 1}-5 f_{z 2}\right\} \sin ^{4} \theta^{*} \\
& F_{02}^{2}=-\frac{1}{8} \sqrt{\frac{3}{2}}\left\{\left(2-2 f_{z 1}-3 f_{z 2}\right)-\left(6-10 f_{z 1}-5 f_{z 2}\right) \cos ^{2} \theta^{*}\right\} \sin ^{2} \theta^{*} \\
& F_{01}^{2}=-\frac{\sqrt{6}}{8}\left\{\left(2-4 f_{z 1}-f_{z 2}\right)-\left(6-10 f_{z 1}-5 f_{z 2}\right) \cos ^{2} \theta^{*}\right\} \cos \theta^{*} \sin \theta^{*} \\
& F_{12}^{2}=\frac{1}{8}\left\{\left(6-6 f_{z 1}-9 f_{z 2}\right)-\left(6-10 f_{z 1}-5 f_{z 2}\right) \cos ^{2} \theta^{*}\right\} \cos \theta^{*} \sin \theta^{*} \\
& F_{-12}^{2}=-\frac{1}{8}\left(6-10 f_{z 1}-5 f_{z 2}\right) \cos \theta^{*} \sin ^{3} \theta^{*},
\end{aligned}
$$

where all parameters contribute and again $f_{00}$ can be expressed as $f_{00}=\left(1-f_{++}-\right.$ $\left.f_{--}-2 f_{+0}-2 f_{0-}-2 f_{+-}\right)$.

While Eq. 4.34 is a cumbersome but general result, in many cases the parameters are zero and many of the terms may be ignored. To provide an example, we explore the case of spin-zero, -one, and -two resonances. The allowed (non-zero) helicity states are given in Table 4.2 where the allowed production and decay helicity fractions are also listed in the two right-most columns. From Table 4.2, we see that if we restrict our discussion to certain spin $J$ resonances, the expression in Eq. 4.34 may become simpler as many of the terms vanish. Recall that due to the decay of identical $Z$ bosons, we can relate some of the helicity amplitudes as shown in the table via Eq. 4.12: $A_{k l}=(-1)^{J} A_{l k}$. 


\section{CHAPTER 4. KINEMATICS OF GENERIC RESONANCES}

Table 4.2: Summary of allowed helicity states for spin-zero, -one and -two resonances.

\begin{tabular}{|c|c|c|c|c|}
\hline spin & production & polarization & production fractions & helicity amplitudes \\
\hline spin-zero & $g g$ & 0 & $f_{z 0}$ & $A_{++}, A_{--}, A_{00}$ \\
\hline spin-one & $q \bar{q}$ & \pm 1 & $f_{z 1}$ & $\begin{array}{l}A_{+0}=A_{0+} \\
A_{0_{-}}=A_{-0}\end{array}$ \\
\hline spin-two & $g g$ or $q \bar{q}$ & $0, \pm 1, \pm 2$ & $f_{z 0}, f_{z 1}, f_{z 2}$ & $\begin{array}{c}A_{++}, A_{--}, A_{00} \\
A_{+0}=A_{0+} \\
A_{0-}=A_{-0} \\
A_{+-}=A_{-+}\end{array}$ \\
\hline
\end{tabular}

It is interesting to note that in Eq. 4.38, there is often a linear combination of terms of the form $\left(\alpha_{1}+\alpha_{2} f_{z 1}+\alpha_{3} f_{z 2}\right)$, multiplied by other parameters. It is peculiar that in all $\theta^{*}$ - and $\Phi_{1}$-dependent terms, there is a relationship between the $\alpha$-coefficients $\alpha_{1}=-0.4 \times\left(\alpha_{2}+\alpha_{3}\right)$. This means that if we choose $f_{z 1}=f_{z 2}=0.4$ in Eq. 4.38 , the production angle distribution for the spin-two particle becomes flat! To understand this, note that those helicity fractions imply production of an unpolarized $X$-boson in which case the production angle distribution must be constant. It is interesting to point out in this regard that observation of the flat production angle distribution in a two-body decay $X \rightarrow P_{1} P_{2}$ does not mean that the $X^{\prime}$ s spin is zero. In fact, $X$ can have any spin $J$ but, for the flat distribution, it must be produced unpolarized. In turn, this implies that all helicity fractions must be equal $2 f_{z 0}=f_{z 1}=f_{z 2}=\ldots . f_{z J}$. 


\section{CHAPTER 4. KINEMATICS OF GENERIC RESONANCES}

\section{Analysis of $Z Z^{*}$ resonances}

So far, we have assumed a search for a resonance decaying to two real on-shell $Z$ bosons whose mass is greater than $2 m_{Z}$, in other words above the $Z Z$ mass threshold. Under this assumption, the helicity amplitudes $A_{\lambda_{1} \lambda_{2}}$ are good experimental observables in the case of the narrow-mass approximation. This is a good approximation for the narrow resonances $X$ and $Z$ with a mass above the $Z Z$ mass threshold and we can assume the mass distributions of the $X$ and $Z$ 's are uncorrelated to the angular distributions. However, below the $Z Z$ mass threshold, the helicity amplitudes depend on the actual mass of the two daughters in the decay $X \rightarrow Z Z^{*}$ where one of the $Z$ bosons is now virtual and off-shell. Then, a correlated mass and angular distribution must be developed. As a motivated example, we take the case of the spin-zero $X$ particle since much of the phenomenological interest in a resonance below the $Z Z$ threshold comes from a Higgs-like spin-zero particle. Its coupling to two $Z$ bosons is generally described as in Eq. 4.1. The helicity amplitudes are recalculated as follows

$$
\begin{aligned}
& A_{00}=-\frac{m_{X}^{2}}{v}\left(a_{1} \chi+a_{2} \eta\left(\chi^{2}-1\right)\right) \\
& A_{ \pm \pm}=\frac{m_{X}^{2}}{v}\left(a_{1} \pm \frac{i a_{3} \eta \sqrt{\chi^{2}-1}}{2}\right)
\end{aligned}
$$

where the parameters $\chi$ and $\eta$ now depend on the masses of the particles, and in particular on the mass $m_{*}$ of the off-shell $Z^{*}$

$$
\begin{aligned}
\chi & =\left(m_{X}^{2}-m_{Z}^{2}-m_{*}^{2}\right) /\left(2 m_{Z} m_{*}\right) \\
\eta & =m_{Z} m_{*} / m_{X}^{2}
\end{aligned}
$$




\section{CHAPTER 4. KINEMATICS OF GENERIC RESONANCES}

The angular distribution would still be described by Eq. (4.34), but the values of $f_{\lambda_{1} \lambda_{2}}$ are no longer good quantities to fit for since they are not constant. Instead, $f_{\lambda_{1} \lambda_{2}}$ should be substituted by $\left|A_{\lambda_{1} \lambda_{2}}\right|^{2}$ expressed through coupling constants and masses using Eq. 4.39. Therefore, the 5D angular distrubution becomes 6D mass-nagular distribution where dependence on $m_{*}$ enters in a correlated way.

We define the dimensionless coupling constants $a_{i}=a_{1}, a_{2}, a_{3}$ as $a_{i}=\left|a_{i}\right| e^{i \phi_{i}}$. We can see the dependence of the angular distribution on the off-shell boson mass $m_{*}$ through $\eta, \chi$ by writing down the free parameters:

$$
\begin{aligned}
\left|A_{00}\right|^{2} & =\frac{M_{X}^{4}}{v^{2}}\left\{\left|a_{1}\right|^{2} \chi^{2}+\left|a_{2}\right|^{2} \eta^{2}\left(\chi^{2}-1\right)^{2}+2\left|a_{1}\right|\left|a_{2}\right| \chi\left(\chi^{2}-1\right) \eta \cos \left(\phi_{1}-\phi_{2}\right)\right\} \\
\left|A_{ \pm \pm}\right|^{2} & =\frac{M_{X}^{4}}{v^{2}}\left\{\left|a_{1}\right|^{2}+\left|a_{3}\right|^{2} \eta^{2}\left(\chi^{2}-1\right) \pm 2\left|a_{1}\right|\left|a_{3}\right| \chi \eta \sqrt{\chi^{2}-1} \cos \left(\phi_{1}-\phi_{3}\right)\right\} \\
\phi_{00} & =\arctan 2\left[\left|a_{1}\right| \chi \cos \phi_{1}+\left|a_{2}\right| \eta\left(\chi^{2}-1\right) \cos \phi_{2},\left|a_{1}\right| \chi \sin \phi_{1}+\left|a_{2}\right| \eta\left(\chi^{2}-1\right) \sin \phi_{2}\right] \\
\phi_{ \pm \pm} & =\arctan 2\left[\left|a_{1}\right| \cos \phi_{1} \mp\left|a_{3}\right| \eta \sqrt{\chi^{2}-1} \cos \phi_{3},\left|a_{1}\right| \sin \phi_{1} \pm\left|a_{3}\right| \eta \sqrt{\chi^{2}-1} \sin \phi_{3}\right]
\end{aligned}
$$

To write down the fully differential mass-angle expression, we must add in the $Z^{*}$ propagator term, as in Eq. 23 of Ref. [41].

$\frac{d \Gamma_{J=0}}{\Gamma d\left(M_{*}\right)^{2} d \cos \theta_{1} d \cos \theta_{2} d \Phi} \sim \frac{M_{*}^{2} \beta}{\left(M_{*}^{2}-M_{Z}^{2}\right)^{2}+M_{Z}^{2} \Gamma_{Z}^{2}}\left[\frac{d \Gamma_{J=0}}{\Gamma d \cos \theta_{1} d \cos \theta_{2} d \Phi}\left(M_{*}, \cos \theta_{1}, \cos \theta_{2}, \Phi\right)\right]$

where we have defined

$$
\beta^{2}=\left[1-\frac{\left(M_{Z}+M_{*}\right)^{2}}{M_{X}^{2}}\right]\left[1-\frac{\left(M_{Z}-M_{*}\right)^{2}}{M_{X}^{2}}\right] .
$$

As a cross-check, in Fig. 4.5 we show the invariant mass distribution for the virtual $Z$ by integrating out the angular contribution for the case of a scalar $(\mathrm{H})$ and 


\section{CHAPTER 4. KINEMATICS OF GENERIC RESONANCES}

pseudoscalar (A) spin-zero particle at $140 \mathrm{GeV}$. This is equivalently the distribution of $\left(\left|A_{++}\right|^{2}+\left|A_{--}\right|^{2}+\left|A_{00}\right|^{2}\right)$ with the mass dependence expressed through Eq. 4.39. For the case of a scalar spin-zero, we set $a_{1}=1$ and $a_{2}=a_{3}=\phi_{i}=0$. For the case of a pseudoscalar spin-zero, we set $a_{3}=1$ and $a_{1}=a_{2}=\phi_{i}=0$. The results in Fig. 4.5 agree with the results in Fig. 11 of Ref. [41].

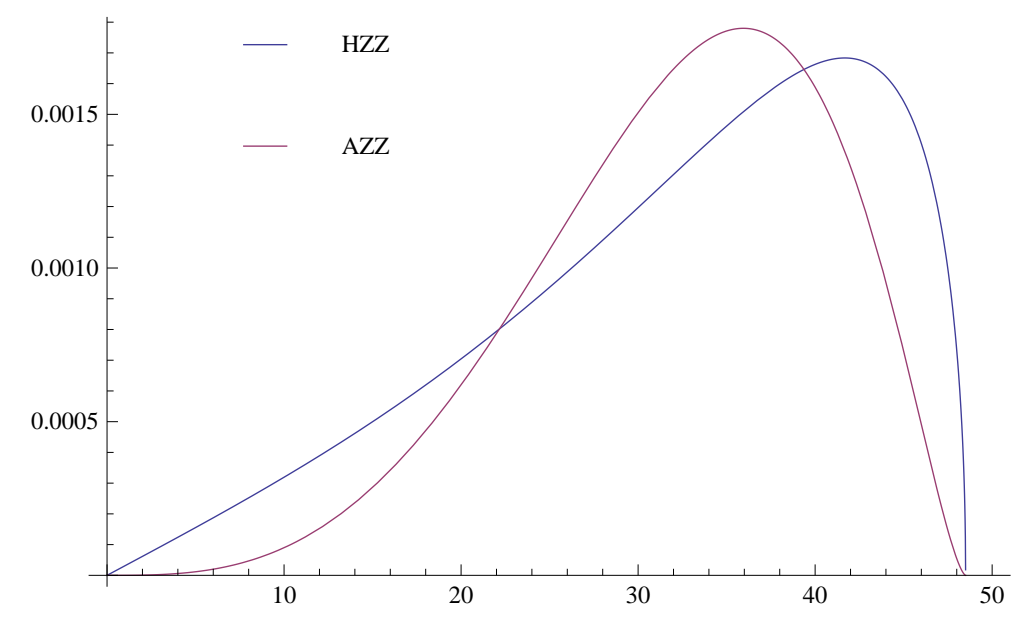

Figure 4.5: Virtual $Z$ invariant mass, $m_{*}$, for a scalar and pseudoscalar spin-zero particle.

\subsection{Applications at the LHC}

In the previous section, the angular distributions for a general spin $J$ resonance decaying in either a two-body or four-body final state are calculated. We use the helicity amplitude formalism introduced in Chapter 2 and have expanded it in this chapter to parameterize those angular distributions. The helicity amplitude formalism 


\section{CHAPTER 4. KINEMATICS OF GENERIC RESONANCES}

is developed in a way to be model-independent and only general couplings are used. The angular distributions describe the kinematics and phase space of a given process regardless of the final state.

In the following chapters we apply the general concepts and distributions from this chapter to two specific analyses at the LHC. In both cases, while the kinematics are fully described in the above sections, real life is more complex due to detector effects such as a detector acceptance which sculpts the angular distributions, finite detector resolution and backgrounds. In the Chapter 5 we look at the SM process of a $\gamma$ or $Z$ decaying to muons. In this decay mode, the angular distribution can be used to directly measure the couplings of the $Z$ boson and measure the SM parameter $\sin ^{2} \theta_{W}$. In Chapter 6 , the golden mode $X \rightarrow Z Z \rightarrow 4 l$ is used to differentiate between different possible signal hypotheses. If a new resonance is discovered at the LHC, the next task will be to understand the nature of such a resonance and its how it couples to the SM fields. 


\section{Chapter 5}

\section{Measurement of the Weak Mixing}

\section{Angle}

"Her personality is like a three. Her sense of humor is a two. Her ears are like a seven, and a four. Add it all up and what do you get? 16. And he treats her like she's a perfect forty. "

- Kelly Erin Hannon, The Office

In Section 2.3, the SM parameter $\sin ^{2} \theta_{W}$ was introduced. Recall $\theta_{W}$ is the weak mixing angle which defines the electroweak boson couplings and the rotation in the $S U(2)_{L} \times U(1)_{Y}$ electroweak symmetry. In order to measure the weak mixing angle, we examine the SM Drell-Yan production of the $Z$ and $\gamma^{*}$ in $q \bar{q}$ annihilation and subsequent decay to a di-lepton pair [42]. The Feynman diagram for the process is pictured in Fig. 5.1. In Section 4.4, we developed the angular formalism for a generic two-body final state resonance at the LHC. We can use this description of 


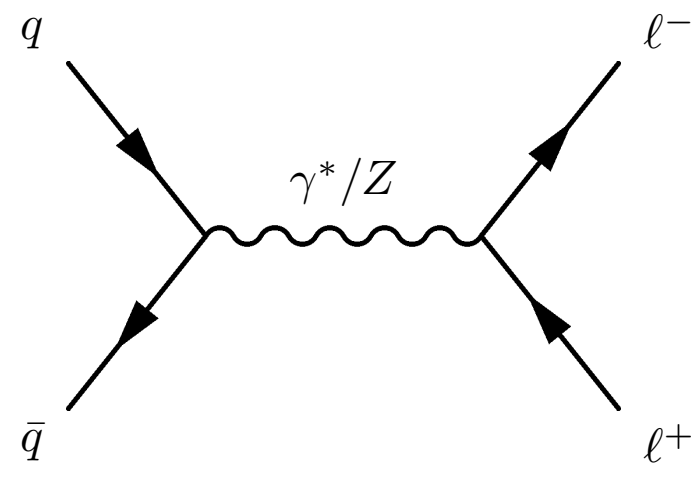

Figure 5.1: Drell-Yan process

the kinematics in the Drell-Yan process as a model to test our formalism to determine quantum numbers and couplings of a resonance by using the well-established $Z$ boson. The process $X \rightarrow l^{+} l^{-}$has been well studied in the literature [43-49]. Recently, Ref. [33] extended the angular analysis formalism to allow for different resonance $X$ polarization in production.

Couplings of the $Z$ to a lepton pair have also been measured with high precision by the dedicated $e^{+} e^{-}$LEP and SLC experiments [50]. However, couplings to the light quarks, such as $Z u \bar{u}$ and $Z d \bar{d}$ are known to less precision. For example, there was a lot of excitement when the NuTeV collaboration measured the weak mixing angle $\sin ^{2} \theta_{W}=0.2277 \pm 0.0016$ which was $3.0 \sigma$ away from the SM prediction [51]. The best Tevatron measurement [52] at the $Z$ has a slightly better precision. Both measurements are sensitive to the $Z q \bar{q}$ coupling.

The proton-proton collisions at the LHC pose new challenges compared to previous experiments. The presence of both the axial-vector and vector couplings leads to an 


\section{CHAPTER 5. MEASUREMENT OF THE WEAK MIXING ANGLE}

asymmetry in the distribution of the angle $\cos \theta^{*}$ illustrated in Fig. 5.2. This 'forwardbackward' asymmetry, $A_{\mathrm{FB}}$, has been the primary measurement to extract the couplings and $\sin ^{2} \theta_{W}$ at the LEP, SLC, and Tevatron experiments. However, one added challenge at the LHC is the direction of the quark direction can be deduced only on a statistical basis [44] using the boost direction of the final state.

Therefore, we develop a per-event multivariate analytical likelihood which uses full information about the Drell-Yan process $q \bar{q} \rightarrow \gamma^{*} / Z \rightarrow \ell^{-} \ell^{+}$parameterized as a function of the dilepton rapidity $Y$, dilepton invariant mass squared $s$, and dilepton decay angle $\theta^{*}$. This technique uses more information than the conventional forwardbackward asymmetry approach and the distribution of the polar angle as a function of both mass and rapidity is an essential component of this method. This technique is applied for the high-precision electroweak measurement of the weak mixing angle, but it also applies to searches for new resonances at the LHC. Our approach in this analysis is to perform a blind fit of the weak mixing angle. Blind fits are a common analysis strategy and keep the analyzers unbiased from any preconceptions on what the results should be. The final fit result of $\sin ^{2} \theta_{W}$ to the CMS data is kept blind until a review of the entire analysis is complete including evaluation of systematic effects though we do look at projections of the observables and the errors from the fit. 


\subsection{Analytical Parameterization of the Drell- Yan Process}

The philosophy of the multivariate likelihood analysis is to first write the phenomenological model of the process and then introduce detector effects into the model to match the observed data. The application of the multivariate likelihood method will be described in greater detail later. Parameters of the model may either be fixed to the best known values or left free in the fit and determined from the data. These parameters may include the physical quantities of interest, such as $\sin ^{2} \theta_{W}$, or a description of the detector effects, such as a correction for the energy scale in the track reconstruction. Therefore, we start with discussion of the phenomenological model, and then proceed to detector-specific effects in the application of the analysis to CMS data.

The description of the phenomenological model is built from a leading order (LO) description of the Drell-Yan process illustrated in Fig. 5.1. In reality, we expect higherorder corrections to contribute to this process from strong (QCD) and electroweak (EWK) interactions. Two examples include: hard gluon radiation from the initial state quarks (ISR) which may give the dilepton system a significant transverse momentum component and distort LO angular distributions and final state radiation of photons from the final state leptons (FSR) which causes the reconstructed mass to be less than intermediate resonance invariant mass. We consider these higher-order processes as 
corrections to our LO model and are studied with detailed next-to-leading order (NLO) simulation.

\section{Weak boson couplings to fermions}

To derive the angular distribution of the Drell-Yan process, we use the helicity amplitude formalism developed in Chapter 4. We need to derive the helicity amplitudes for the SM Drell-Yan case of the spin-one resonance. Recall Eq. 4.8 which gives the general amplitude of a spin-one resonance to fermions. Starting from this amplitude, we can derive the various helicity amplitudes which have already been given in Section 4.3. In the relativistic limit of light $\left(m_{q} \rightarrow 0\right)$ quarks $q \bar{q} \rightarrow X$, a spin-zero polarization on the production axis is not possible. Therefore, only the $\rho_{1}^{(J)}$ and $\rho_{2}^{(J)}$ coupling constants can contribute in the production of the spin-one resonances.

From the discussion in Section 2.3, the $Z$ neutral current interaction was introduced in Eq. 2.26. In this equation, $c_{V}^{f}=T_{f}^{3}-2 \sin ^{2} \theta_{W} Q_{f}$ and $c_{A}^{f}=T_{f}^{3}$ are defined as the vector and axial-vector couplings where $T_{f}$ and $Q_{f}$ are the fermion weak isospin and charge quantum numbers, respectively; the SM quantum numbers are given in Table 2.1 Bringing together Eq. 4.8 and Eq. 2.26, it is clear how we can derive the helicity amplitudes for the couplings of the SM $\gamma$ and $Z$ to fermions. In Table 5.1, we explicitly write out the neutral current couplings to fermions in the SM. Note that in the generic formalism the couplings are denoted as $\rho_{1}^{(1)}$ and $\rho_{2}^{(1)}$ while in the $\mathrm{SM}$, those couplings are known as $c_{V}$ and $c_{A}$, respectively. 
Table 5.1: Relative values of standard model neutral current couplings to fermions [61].

\begin{tabular}{lcc}
\hline \hline process & $\rho_{1}^{(1)}=c_{V}$ & $\rho_{2}^{(1)}=c_{A}$ \\
\hline$\gamma \rightarrow l^{+} l^{-}$ & $-e$ & 0 \\
$\gamma \rightarrow u \bar{u}$ & $+2 e / 3$ & 0 \\
$\gamma \rightarrow d \bar{d}$ & $-e / 3$ & 0 \\
$Z \rightarrow l^{+} l^{-}$ & $\frac{-3+12 \sin ^{2} \theta_{W}}{6 \sin \left(2 \theta_{W}\right)} e \simeq-0.045 e$ & $\frac{+1}{2 \sin \left(2 \theta_{W}\right)} e \simeq+0.593 e$ \\
$Z \rightarrow u \bar{u}$ & $\frac{+3-8 \sin ^{2} \theta_{W}}{6 \sin \left(2 \theta_{W}\right)} e \simeq+0.227 e$ & $\frac{-1}{2 \sin \left(2 \theta_{W}\right)} e \simeq-0.593 e$ \\
$Z \rightarrow d \bar{d}$ & $\frac{-3+4 \sin ^{2} \theta_{W}}{6 \sin \left(2 \theta_{W}\right)} e \simeq-0.410 e$ & $\frac{+1}{2 \sin \left(2 \theta_{W}\right)} e \simeq+0.593 e$ \\
\hline \hline
\end{tabular}

From Table 5.1, we have worked out the couplings necessary to define the helicity amplitudes in the SM case. Using these values, we are ready to calculate the angular distributions for the Drell-Yan process.

\section{Angular Distribution}

Starting with Eq. 4.21, we have the general angular distribution for a $2 \rightarrow 2$ process. The production angle $\theta^{*}$ is defined as the angle between the parton collision axis $z$ and the $X$ decay axis $z^{\prime}$ in the $X$ rest frame, see Fig. 5.2. The axis directions are defined by the direction of a fermion (as opposed to antifermion). This is complicated at the LHC where we have proton-proton collisions and the direction of the quark is unknown. This dilution effect is discussed in more detail below. A further complication 


\section{CHAPTER 5. MEASUREMENT OF THE WEAK MIXING ANGLE}

is that determination of the $z$ axis requires care since it may differ somewhat from the beam collision axis due to transverse momentum of the dilepton system from NLO effects. We use the Collins-Soper frame [53] to reduce the uncertainties due to the transverse momentum of the incoming quarks. In this frame, the angle $\theta^{*}$ is defined to be the angle between the lepton momentum and a $z$-axis that bisects the directions of one proton and the opposite direction of the other proton in the $Z$ rest frame. We first discuss angular distributions with respect to the "ideal" parton collision axis $z$.

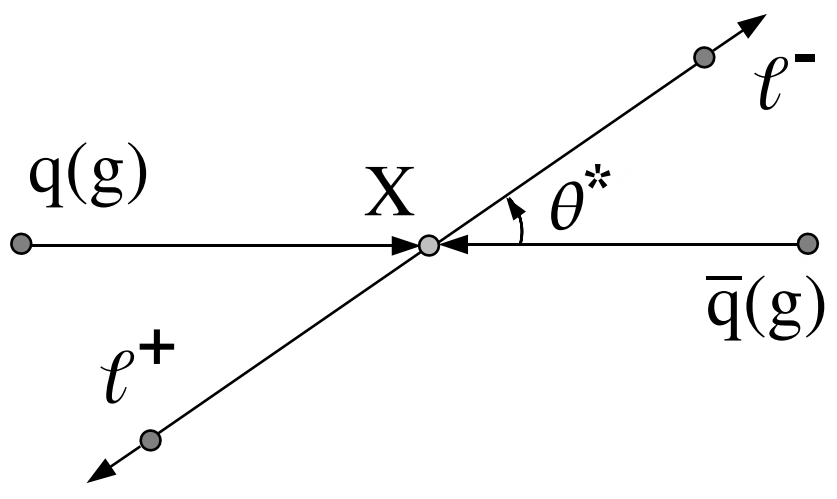

Figure 5.2: The production angle $\theta^{*}$ defined in the $X$ rest frame.

Because we consider the angular observable, $\cos \theta^{*}$ as a function of the dilepton mass, $m$, and the Drell-Yan process involves the interference of two spin-one intermediate states $\left(\gamma^{*} / Z\right)$, the differential cross-section includes mass-dependence in the form of the boson propagator terms.

$$
\begin{aligned}
\hat{\sigma}_{q \bar{q}}\left(\cos \theta^{*}, s\right) & \propto \frac{1}{s} \sum_{\chi_{1}, \chi_{2}, \lambda_{1}, \lambda_{2}=\uparrow, \downarrow}(2 J+1)\left(d_{\chi_{1}-\chi_{2}, \lambda_{1}-\lambda_{2}}^{J=1}\left(\theta^{*}\right)\right)^{2} \\
\times & \left|A_{\lambda_{1}, \lambda_{2}}^{(\gamma \rightarrow l l)} A_{\chi_{1}, \chi_{2}}^{(q \bar{q} \rightarrow \gamma)}+A_{\lambda_{1}, \lambda_{2}}^{(Z \rightarrow l l)}\left(\theta_{W}\right) A_{\chi_{1}, \chi_{2}}^{(q \bar{q} \rightarrow Z)}\left(\theta_{W}\right) \times \frac{s}{\left(s-m_{Z}^{2}\right)+i m_{Z} \Gamma_{Z}}\right|^{2}
\end{aligned}
$$




\section{CHAPTER 5. MEASUREMENT OF THE WEAK MIXING ANGLE}

where $\chi_{1}, \chi_{2}, \lambda_{1}$, and $\lambda_{2}$ are the helicity states of the quark-antiquark and leptonantilepton. The helicity amplitude terms are written in Eq. 5.1 to show that they are dependent on the weak mixing angle $\sin ^{2} \theta_{W}$. In Eq. 5.1, the separate contributions from the virtual photon $\gamma^{*}$ and the $Z$ boson are denoted clearly. The interference of the $\gamma^{*}$ with the $Z$ is clear in the calculation of the differential cross-section. If a new particle such as a new gauge boson is present in a similar process $q \bar{q} \rightarrow Z^{\prime} \rightarrow l^{+} l^{-}$, it would interfere with the $\gamma^{*} / Z$ as well and its presence could be identified using this formalism. A caveat is that a new spin-two resonance produced in the $g g$ initial state would not interfere with the Drell-Yan process.

If we expand out Eq. 5.1, we can see the explicit asymmetric terms

$$
\begin{aligned}
\hat{\sigma}_{q \bar{q}}\left(s, \theta^{*}\right) \propto & \frac{3 c_{V, l}^{(\gamma) 2} c_{V, q}^{(\gamma) 2}}{2 s}\left(1+\cos ^{2} \theta^{*}\right) \\
& +\frac{3}{2} \frac{s}{\left(m_{Z}^{2}-s\right)^{2}+m_{Z}^{2} \Gamma_{Z}^{2}(s)}\left[\left(c_{A, l}^{2}+c_{V, l}^{2}\right)\left(c_{A, q}^{2}+c_{V, q}^{2}\right)\left(1+\cos ^{2} \theta^{*}\right)+8 c_{A, l} c_{V, l} c_{A, q} c_{V, q} \cos \theta^{*}\right] \\
& +\frac{3\left(s-m_{Z}^{2}\right) c_{V, l}^{(\gamma)} c_{V, q}^{(\gamma)}}{\left(m_{Z}^{2}-s\right)^{2}+m_{Z}^{2} \Gamma_{Z}^{2}(s)}\left[c_{V, l} c_{V, q}\left(1+\cos ^{2} \theta^{*}\right)+2 c_{A, l} c_{A, q} \cos \theta^{*}\right]
\end{aligned}
$$

where the axialvector and vector couplings $\left(c_{V}, c_{A}\right)$ are denoted for leptons and quarks $(l, q)$ and the photon couplings are denoted with ${ }^{(\gamma)}$. Eq. 5.2 is written such that the first two lines are the pure photon and $Z$ contributions and the third line is the term arising from the interference of the two bosons. Using the numerical values from Table 5.1, we find the interference term provides the strongest asymmetry.

The above angular distribution in Eq. 5.1 for the SM process $q \bar{q} \rightarrow Z / \gamma^{*} \rightarrow l^{+} l^{-}$ at any given value of $s$ can be rewritten as

$$
\frac{d \Gamma\left(q \bar{q} \rightarrow X_{J=1} \rightarrow P_{1} P_{2}\right)}{\Gamma d \cos \theta^{*}}=\frac{3}{8}\left(1+\cos ^{2} \theta^{*}\right)+A_{F B}^{q \bar{q}}\left(s, \theta_{W}\right) \cos \theta^{*}
$$




\section{CHAPTER 5. MEASUREMENT OF THE WEAK MIXING ANGLE}

which includes the forward-backward asymmetry parameter $A_{F B}$. One can formally define $A_{F B}$ as an asymmetry in the event count with positive and negative values of $\cos \theta^{*}$. From Eq. 5.2 and Table 5.1, it is clear this forward-backward asymmetry depends on the $\mathrm{SM}$ parameter $\sin ^{2} \theta_{W}$, on mass, and on quark type in production, as shown in Fig. 5.3.

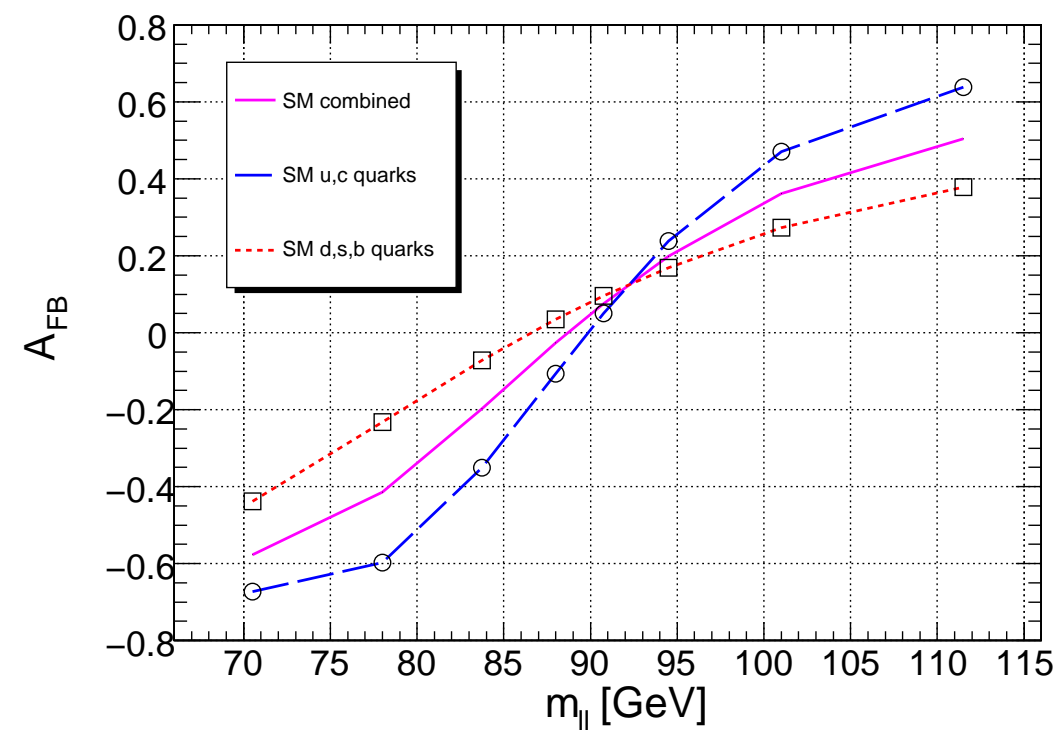

Figure 5.3: Forward-backward asymmetry $A_{F B}$ as a function of mass

\section{Parton distribution functions}

The mass-angle differential cross-section for the Drell-Yan process is given in Eq. 5.2. However, to describe the Drell-Yan process at the LHC, a description of the parton distribution functions (PDFs) is required. The LHC is a proton-proton collider, but our description thus far is built from the quark-antiquark interaction. As was 


\section{CHAPTER 5. MEASUREMENT OF THE WEAK MIXING ANGLE}

discussed briefly in Section 1.1, the proton is composed of valence quarks (two up and one down), gluons, and a sea of all quark flavors. Each proton constituent carries a fraction of the proton total momentum. In Fig. 5.4, an analytical parameterization is given of the PDFs and will be discussed in greater detail below. The parton distribution function is given as a function of the fraction of the proton momentum, $x$, carried by each of the proton constituents. The PDFs are defined for a given parton collision center-of-mass energy, $Q$; this particular plot shows the PDFs for a given $Q=100 \mathrm{GeV}$. We denote the PDFs below as $f\left(x, Q^{2}\right)$.

Because the collisions are proton-proton, the direction of the quark at the LHC is unknown. A solution to this challenge is to choose the $z$ axis direction based on the boost of the dilepton system and the reason is because on average, the quarks carry more of the proton momentum than the antiquarks which belong to the sea. This is clear from Fig. 5.4.

The interaction of two partons can be written in terms of the parton distribution

functions, $f\left(x_{1}, Q^{2}\right)$ and $f\left(x_{2}, Q^{2}\right)$. The rapidity (boost) distribution of the dilepton system, $Y$, is dependent on the fraction of the proton momentum $x$, and thus, the PDFs. The PDFs also contribute to the shaping of the invariant mass distribution, $m_{l l}$. An example of the partonic contributions to the invariant mass distribution is given in Fig 4.1. The contribution to the process from the PDFs is incorporated in the 


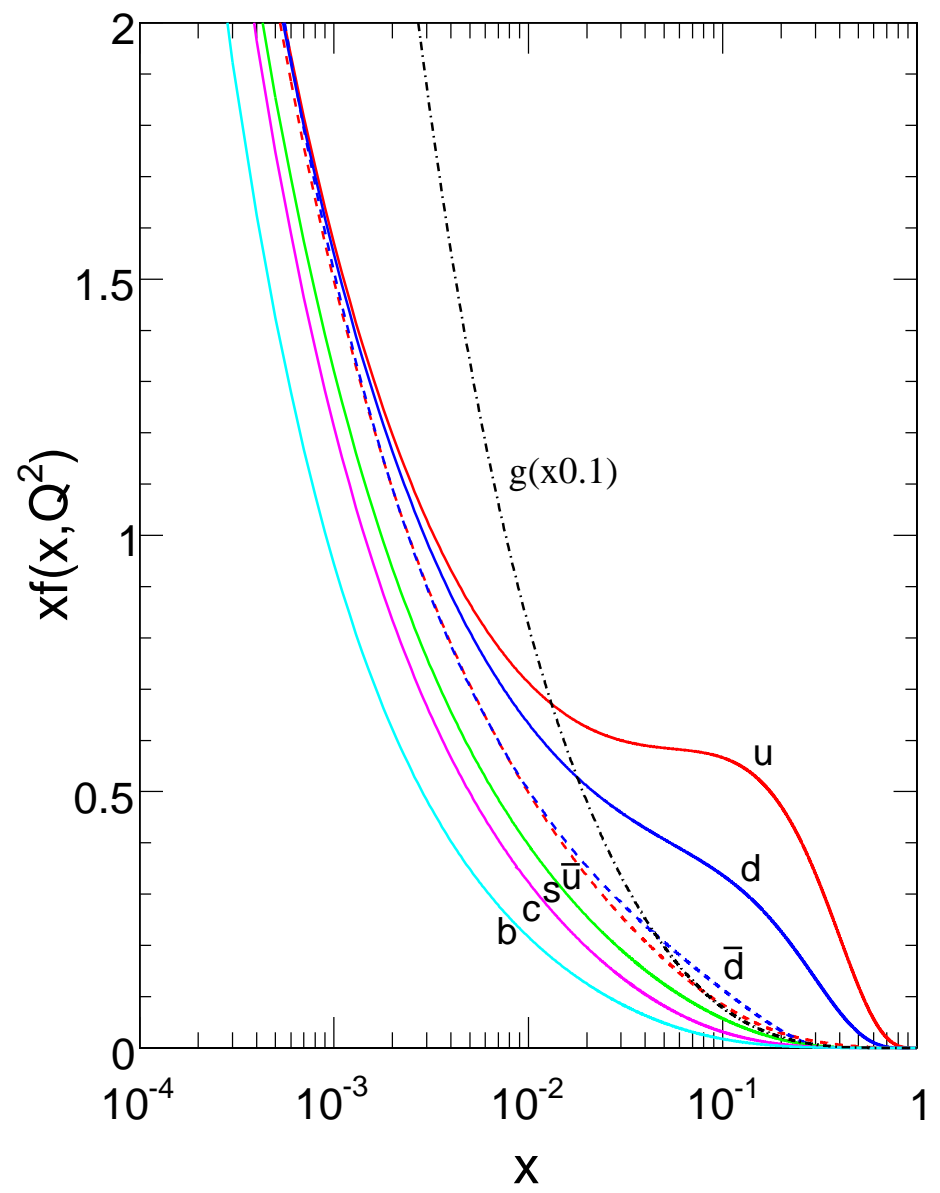

Figure 5.4: Analytical parameterization of the Parton Distribution Functions using the CTEQ6L [54] numerical calculation.

differential cross-section in the following way:

$$
\frac{d \sigma_{p p}\left(p x_{1}, p x_{2}, \cos \theta^{*} ; \theta_{W}\right)}{d x_{1} d x_{2} d \cos \theta^{*}}=\sum_{a b} \hat{\sigma}_{a b}\left(Q^{2}, \cos \theta^{*}\right) \tilde{f}_{a}\left(x_{1}, Q^{2}\right) \tilde{f}_{b}\left(x_{2}, Q^{2}\right)
$$

From Eq. 5.4, the differential cross-section becomes a function of the dilepton invariant mass $m=Q=\sqrt{s}$, the production angle $\theta^{*}$, the parton fraction of the proton momentum $x_{1}$ and $x_{2}$, and the center-of-mass energy of the proton collision which, in the case of the LHC for this analysis, is $\sqrt{s_{p p}}=7 \mathrm{TeV}$. An additional complication, as 


\section{CHAPTER 5. MEASUREMENT OF THE WEAK MIXING ANGLE}

discussed above, is the unknown quark direction which is accounted for on a statistical basis. This is fully described by the PDFs and we express it by incorporating the direction of the dilepton system (boost) to Eq. 5.4 as

$$
\begin{array}{r}
\frac{d \sigma_{p p}\left(p x_{1}, p x_{2}, \cos \theta^{*} ; \theta_{W}\right)}{d x_{1} d x_{2} d \cos \theta^{*}} \propto \sum_{q=u, d, s, c, b}\left(\hat{\sigma}_{q \bar{q}}\left(Q^{2}, \operatorname{sgn}\left(x_{1}-x_{2}\right) \cos \theta^{*} ; \theta_{W}\right) \tilde{f}_{q}\left(x_{1}, Q^{2}\right) \tilde{f}_{\bar{q}}\left(x_{2}, Q^{2}\right)\right. \\
\left.+\hat{\sigma}_{q \bar{q}}\left(Q^{2}, \operatorname{sgn}\left(x_{2}-x_{1}\right) \cos \theta^{*} ; \theta_{W}\right) \tilde{f}_{q}\left(x_{2}, Q^{2}\right) \tilde{f}_{\bar{q}}\left(x_{1}, Q^{2}\right)\right)(5.5)
\end{array}
$$

The sign of the production angle $\cos \theta^{*}$ is defined with respect to the direction of the dilepton system. It is convenient to redefine the two variables $\left(x_{1}, x_{2}\right)$ through $(Y, s)$, the rapidity and the parton center-of-mass energy squared (or momentum transfer $Q^{2}$ ) as

$$
\begin{aligned}
Y & =\frac{1}{2} \ln \frac{E+p_{z}}{E-p_{z}}=\frac{1}{2} \ln \frac{x_{1}}{x_{2}} \\
s & =Q^{2}=x_{1} x_{2} s_{p p}
\end{aligned}
$$

where $\sqrt{s_{p p}}=7 \mathrm{TeV}$ is the LHC center-of-mass energy, $E$ is the system energy, and $p_{z}$ is its longitudinal momentum in the laboratory frame.

After a transformation of the variables, the Drell-Yan process in proton-proton interactions can be expressed as follows

$$
\begin{aligned}
\frac{d \sigma_{p p}\left(Y, s, \cos \theta^{*} ; \theta_{W}\right)}{d Y d s d \cos \theta^{*}} \propto & \sum_{q=u, d, s, c, b}\left[\hat{\sigma}_{q \bar{q}}^{\mathrm{even}}\left(s, \cos ^{2} \theta^{*} ; \theta_{W}\right)\right. \\
& \left.+D_{q \bar{q}}(s, Y) \times \hat{\sigma}_{q \bar{q}}^{\text {odd }}\left(s, \cos \theta^{*} ; \theta_{W}\right)\right] \times F_{q \bar{q}}(s, Y)
\end{aligned}
$$

The parton factor is defined as

$$
\begin{aligned}
F_{q \bar{q}}(s, Y)=\tilde{f}_{q}\left(e^{+Y} \sqrt{s / s_{p p}}, s\right) & \tilde{f}_{\bar{q}}\left(e^{-Y} \sqrt{s / s_{p p}}, s\right) \\
& +\tilde{f}_{q}\left(e^{-Y} \sqrt{s / s_{p p}}, s\right) \tilde{f}_{\bar{q}}\left(e^{+Y} \sqrt{s / s_{p p}}, s\right)
\end{aligned}
$$




\section{CHAPTER 5. MEASUREMENT OF THE WEAK MIXING ANGLE}

and the dilution factor is defined as

$$
\begin{aligned}
& D_{q \bar{q}}(s, Y)= \\
& \frac{\tilde{f}_{q}\left(e^{+|Y|} \sqrt{s / s_{p p}}, s\right) \tilde{f}_{\bar{q}}\left(e^{-|Y|} \sqrt{s / s_{p p}}, s\right)-\tilde{f}_{q}\left(e^{-|Y|} \sqrt{s / s_{p p}}, s\right) \tilde{f}_{\bar{q}}\left(e^{+|Y|} \sqrt{s / s_{p p}}, s\right)}{\tilde{f}_{q}\left(e^{+Y} \sqrt{s / s_{p p}}, s\right) \tilde{f}_{\bar{q}}\left(e^{-Y} \sqrt{s / s_{p p}}, s\right)+\tilde{f}_{q}\left(e^{-Y} \sqrt{s / s_{p p}}, s\right) \tilde{f}_{\bar{q}}\left(e^{+Y} \sqrt{s / s_{p p}}, s\right)} .
\end{aligned}
$$

The partonic cross-section (Eq. 5.2) has been expressed in either even or odd powers of $\cos \theta^{*}$

$$
\begin{aligned}
& \hat{\sigma}_{q \bar{q}}^{\text {even }}\left(s, \cos ^{2} \theta^{*} ; \theta_{W}\right)=\frac{\hat{\sigma}_{q \bar{q}}\left(s,+\cos \theta^{*} ; \theta_{W}\right)+\hat{\sigma}_{q \bar{q}}\left(s,-\cos \theta^{*} ; \theta_{W}\right)}{2}, \\
& \hat{\sigma}_{q \bar{q}}^{\text {odd }}\left(s, \cos \theta^{*} ; \theta_{W}\right)=\frac{\hat{\sigma}_{q \bar{q}}\left(s,+\cos \theta^{*} ; \theta_{W}\right)-\hat{\sigma}_{q \bar{q}}\left(s,-\cos \theta^{*} ; \theta_{W}\right)}{2} .
\end{aligned}
$$

Both factors $F_{q \bar{q}}(s, Y)$ and $D_{q \bar{q}}(s, Y)$ arise from the PDFs and can be extracted from parameterizations such as in Refs. [54-57]. We choose to extract PDFs numerically from the leading order (LO) parameterization CTEQ6 [54] to match the LO model of the process. We then parameterize the PDFs analytically in the relevant range of $x$ for this analysis and with coefficients which are also functions of $Q$. More details of this analytical parameterization are given in a short section below. This analytical parameterization is illustrated in Fig. 5.4 for $Q=100 \mathrm{GeV}$. We choose an analytical parameterization because of the computational speed and ease of implementation, but performance has been cross-checked with numerical computation. For systematic uncertainty studies, we check the performance against simulation with other PDF models [58, 59], such as NLO CTEQ [55], MSTW [56], and NNPDF [57].

The factor $F_{q \bar{q}}(s, Y)$ is the effective cross-section factor which scales the elementary parton-level cross-section. This factor quickly decreases as the energy scale reaches 


\section{CHAPTER 5. MEASUREMENT OF THE WEAK MIXING ANGLE}

values comparable to the full proton energies, as illustrated in Fig. 5.5 for $Y=0$ production. It is shown for the for five quark flavors and also for the gluon contribution scaled by 0.1 to fit in the plot range. The dilution factor $D_{q \bar{q}}(s, Y)$ reflects the fact that the quark direction is generally unknown and is taken as the boost direction of the system, reflecting the higher probability for valence quarks to provide the boost. In Fig. 5.6, the dilution faction is illustrated for $q=u$ (red) or $d$ (blue) in the ranges between 0 and 1 as $|Y|$ changes from 0 to 4 . It is shown for the analytical model (lines) and PYTHIA simulation (data points) with good agreement. From Eq. 5.10 it follows that $D=0$ for $q=s, c, b$ under the assumption $f_{\bar{q}}\left(x, Q^{2}\right)=f_{q}\left(x, Q^{2}\right)$, which is a good approximation in the current PDF model. The challenge at LHC is that the dilution factor $D_{q \bar{q}}$ is small for the typical range of $Y$ values in the detector acceptance region. Information about $\sin ^{2} \theta_{W}$ or individual fermion couplings is contained in the shape of the 3D distributions in Eq. 5.8 and enters through elementary couplings of the electroweak bosons and fermions in the process $q \bar{q} \rightarrow \gamma^{*} / Z \rightarrow \ell^{-} \ell^{+}$.

Figure 5.7 illustrates projections of the correlated distribution from Eq. 5.8 in $Y$ (top), $m$ (middle), and $\cos \theta^{*}$ (bottom). The analytical model in Eq. 5.8 shows good agreement with conventional leading order numerical simulation using the PYTHIA generator [60] with LO CTEQ6 [54] PDFs. The five quark flavors are shown combined (black circles) and separately, in order of decreasing contribution: $u \bar{u}$ (fraction 0.450, shown as red boxes), $d \bar{d}(0.375$, blue crosses), $s \bar{s}(0.103$, green dimonds), $c \bar{c}(0.048$, magenta triangles), and $b \bar{b}(0.025$, cyan stars), where numbers in parentheses indicate 
CHAPTER 5. MEASUREMENT OF THE WEAK MIXING ANGLE

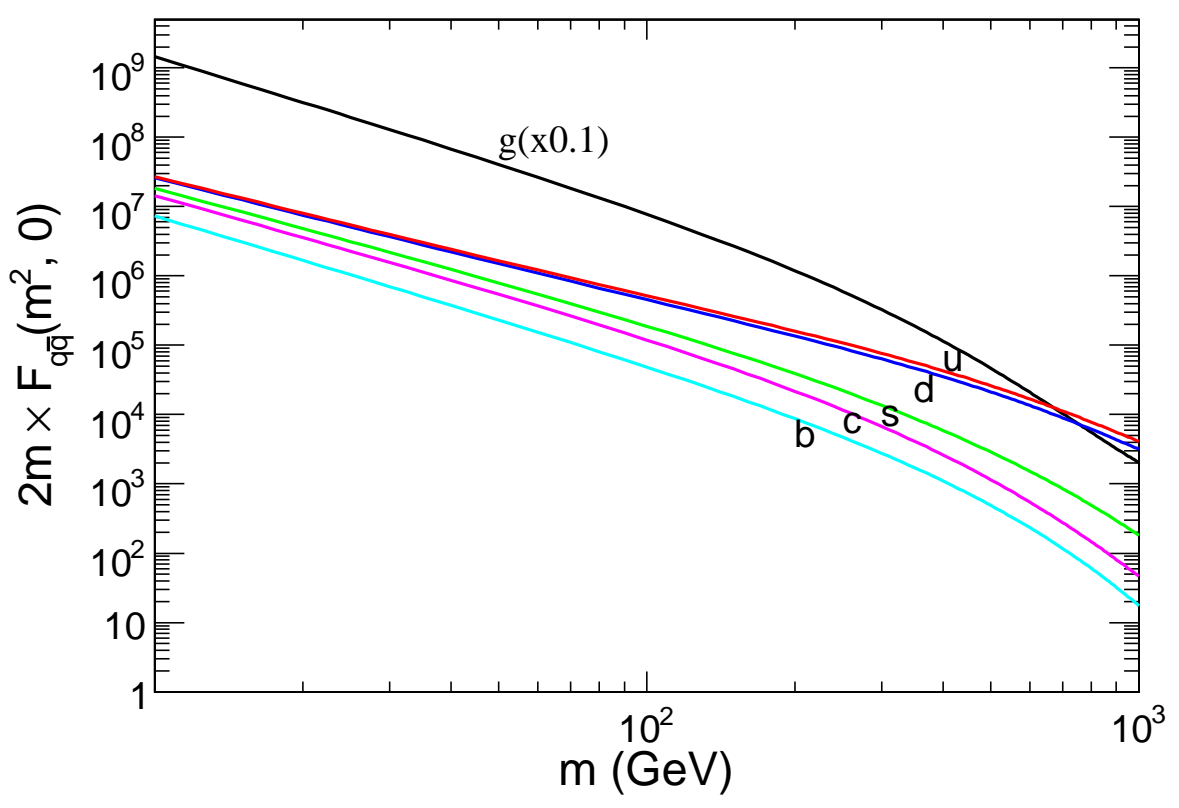

Figure 5.5: Distribution of the effective cross-section factor $F_{q \bar{q}}\left(m^{2}, Y=0\right)$.

fractions.

As mentioned above, the above cross-section is parameterized at the leading order in both strong $(\mathrm{QCD})$ and electroweak (EWK) interactions. Effects from the NLO QCD contributions are studied with a detailed NLO simulation. Effects from NLO EWK contributions are expected to be small compared to precision in this analysis. Electroweak corrections are absorbed in an effective definition of the weak-mixing parameter, $\sin ^{2} \theta_{\text {eff }}$ [61]. We use $\sin ^{2} \theta_{\text {eff }}$ in place of $\sin ^{2} \theta_{W}$ in the rest of this paper. 


\section{CHAPTER 5. MEASUREMENT OF THE WEAK MIXING ANGLE}

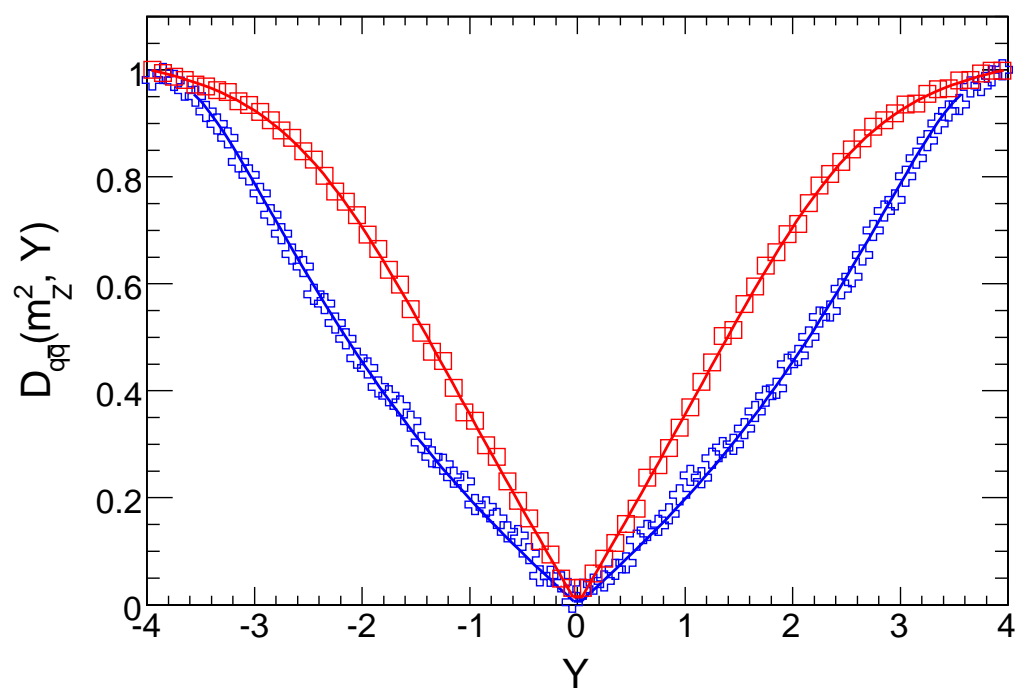

Figure 5.6: Distribution of the dilution factor $D_{q \bar{q}}\left(m=m_{Z}, Y\right)$.

\section{Analytical parameterization of the PDFs}

Analytical parameterization of the ideal distribution $\mathcal{P}_{\text {ideal }}\left(Y, m, \cos \theta^{*}\right)$ in Eq. 5.8 requires knowledge of the parton distributions functions $\tilde{f}_{a}\left(x, Q^{2}\right)$ for each parton type $a$. In order to obtain an analytical function of two variables, we first parameterize $x \tilde{f}_{a}\left(x, Q^{2}\right)$ in slices of $Q^{2}$. We extract 100000 bins between $x=0$ and 1 from CTEQ6 [?] parameterization and plot $x \tilde{f}_{a}\left(x, Q^{2}\right)$ for $a=u, d, \bar{u}, \bar{d}, s, c, b$ quarks (and in principle for gluons $a=g$ ). Note that distributions for sea quarks and antiquarks are expected to be the same (for $s, c$, or $b$ and for $\bar{s}, \bar{c}$, or $\bar{b}$ ). At this point we do not worry about systematic uncertainties in these distributions, but later compare results to expectation.

We consider five mass points corresponding to $Q=50,100,300,500$, and $1000 \mathrm{GeV}$, 


\section{CHAPTER 5. MEASUREMENT OF THE WEAK MIXING ANGLE}
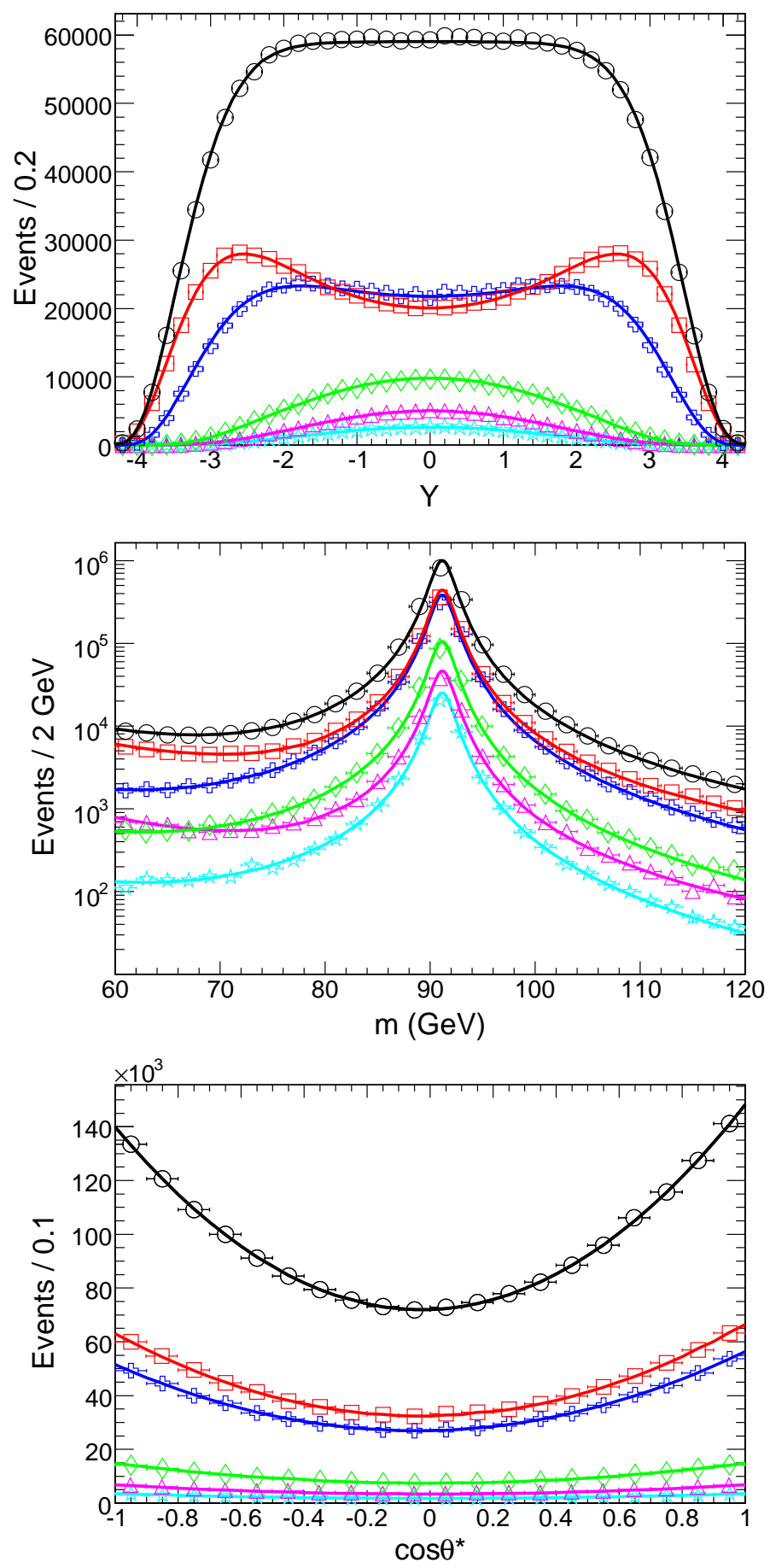

Figure 5.7: Distributions of $Y, m, \cos \theta^{*}$ for the analytical model and PYTHIA simulation for individual quark flavor contributions. 


\section{CHAPTER 5. MEASUREMENT OF THE WEAK MIXING ANGLE}

where the wide range of $Q$ values is considered for good interpolation of parameters across the wide range of reconstructed masses. At each value of $Q$, we parameterize the $x$ dependence with coefficients $a_{i}$ which also depend on $Q$ as follows

$$
\begin{aligned}
x \tilde{f}_{a}\left(x, Q^{2}\right)=\left[a_{0}(Q)\right. & \left.+a_{1}(Q) \times x+a_{2}(Q) \times x^{2}+a_{3}(Q) \times x^{3}\right] \\
& \times(1-x)^{4} \times x^{a_{4}(Q)} \times \exp \left(1+a_{5}(Q) \times x\right)
\end{aligned}
$$

The parton distribution functions are illustrated for up quarks at $Q=100 \mathrm{GeV}$ in Fig. 5.8 [?]. On the left, the quantity $x \tilde{f}_{u}(x)$ is compared from the numerical computation CTEQ6L as data points versus the analytical parameterization (lines). The same comparison is made on the right with different axis scales. Good agreement is observed where the initial uncertainties on the points were $5 \%$ and the fit results were consistent with this error estimate. We parameterize dependence of each coefficient $a_{i}(Q)$ on $Q$, as shown for an example of the first two coefficients in the up quark pa-

rameterization in Fig. 5.9. As a result, we get the full parameterization of $x \tilde{f}_{a}\left(x, Q^{2}\right)$ for the range of $Q$ values from 50 to $1000 \mathrm{GeV}$. The range of the $x$ values considered depends somewhat on the rapidity distributions in the detector acceptance, discussed below, and the $Q$ value, but it typically covers the range from $10^{-4}$ to 0.8 .

\subsection{Event Reconstruction}

In the previous section, an analytical parameterization of the Drell-Yan process dependent on the effective weak mixing angle is presented. We now apply our ana- 
CHAPTER 5. MEASUREMENT OF THE WEAK MIXING ANGLE
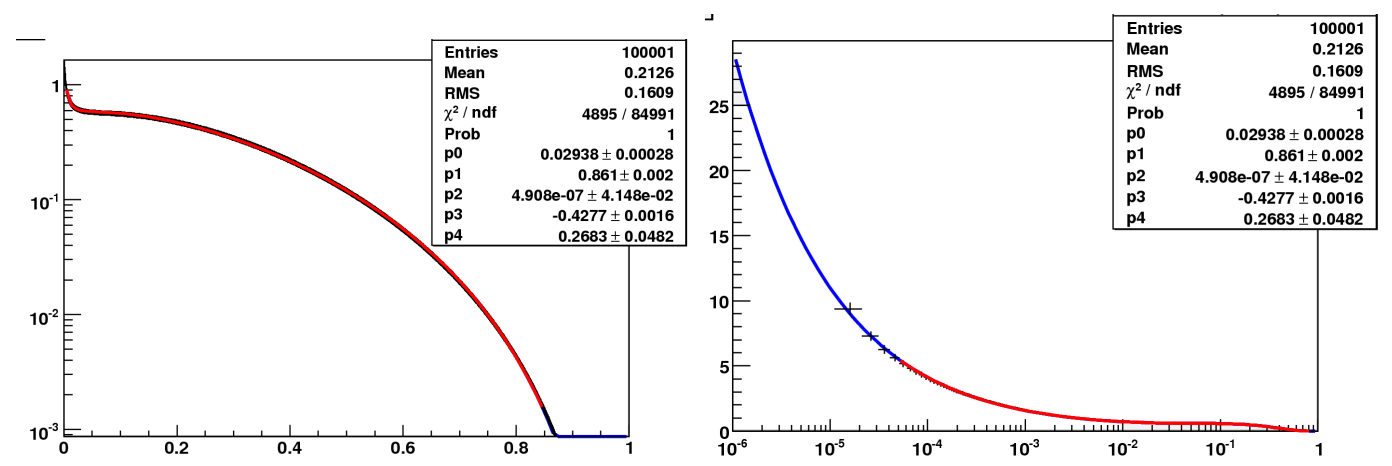

Figure 5.8: Comparison of numerical PDF computation CTEQ6L versus the analytical description.
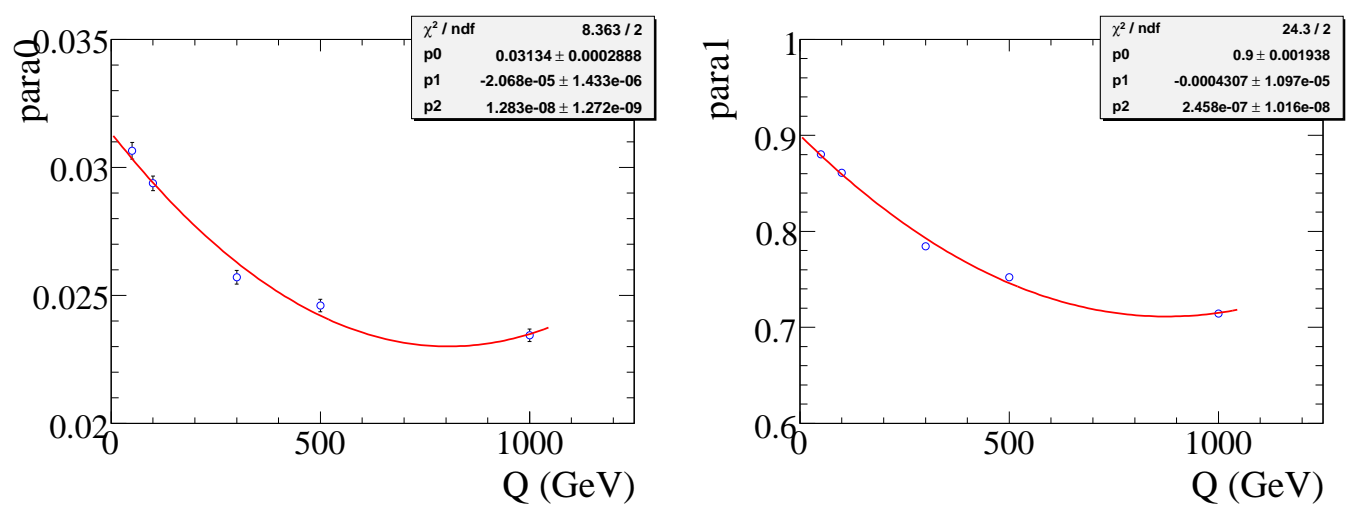

Figure 5.9: $Q$-dependence of the PDF fit parameters.

lytical description of the $q \bar{q} \rightarrow \gamma^{*} / Z \rightarrow \mu^{-} \mu^{+}$process to measure $\sin ^{2} \theta_{\text {eff. }}$ The choice of $\mu^{-} \mu^{+}$, as opposed to $e^{-} e^{+}$, is motivated by the simpler description of the CMS detector and background effects and by the fact that this final state has not yet been studied for $\sin ^{2} \theta_{\text {eff }}$ measurements in $q \bar{q}$ interactions. However, there is no expected limitation in the method for future application to other final states.

The expected multivariate distributions in Eq. 5.8 are modified by smearing due to detector resolution and photon final-state radiation and by non-uniform acceptance 


\section{CHAPTER 5. MEASUREMENT OF THE WEAK MIXING ANGLE}

effects over the phase-space of the observables. These effects will be accounted for in the analysis strategy in the next section.

The analysis uses the CMS [62] data corresponding to $(1.07 \pm 0.05) \mathrm{fb}^{-1}$ of integrated luminosity of the LHC proton-proton collisions at a center of mass energy of $\sqrt{s_{p p}}=7 \mathrm{TeV}$ collected during 2010 and 2011. The signal process $q \bar{q} \rightarrow \gamma^{*} / Z \rightarrow \mu^{-} \mu^{+}$ and the background process $q \bar{q} \rightarrow \gamma^{*} / Z \rightarrow \tau^{-} \tau^{+}$have been simulated with the NLO QCD generator POWHEG [63-65]. Parton showering is simulated using PYTHIA. The NLO PDFs used are CT10 [55]. Background samples of $W+$ jets and $t \bar{t}$ are generated using MadGraph [66], PYTHIA, and TAUOLA [67]. Backgrounds from $W W, W Z, Z Z$, and QCD are generated using PYTHIA. Generated events are processed through the CMS detector simulation and reconstruction. The simulation is created to most realistically represent the conditions of the detector during data collection. The CMS detector simulation is based on Geant $4[68,69]$.

Muon candidates are selected from an trigger-selected sample that contains events with two muons in the volume $|\eta| \leq 2.4$ with a transverse momentum $\left(p_{T}\right)$ requirement. This requirement depends on the period of data-taking, however it always accepts two muons with $p_{T}$ of at least 8 and $13 \mathrm{GeV}$. Offline, muon tracks are first reconstructed independently in the Tracker and the Muon System. Muon candidates are then reconstructed by two different algorithms. The global muon algorithm matches tracks in the Tracker to tracks in the Muon System, and then refits the individual hits in Tracker and Muon System to one overall track. The Tracker muon algorithm extrapo- 


\section{CHAPTER 5. MEASUREMENT OF THE WEAK MIXING ANGLE}

lates tracks with $p_{T}>0.5 \mathrm{GeV}$ and $p>2.5 \mathrm{GeV}$ in the Tracker to the Muon System, and a track is taken to be a muon candidate if it matches at least one track segment in the Muon System. Both algorithms take into account energy loss and multiple scattering in the yoke steel of the CMS magnet. High-quality muons are selected using standard CMS muon identification requirements. These criteria demand 10 hits in the Tracker, including one in the pixel detector, at least one hit in the muon stations, and a normalized $\chi^{2}<10$ for the global fit.

Muons are required to have a small impact parameter of less than $2 \mathrm{~mm}$ measured with respect to the beam spot. We further require the angle between the two muon tracks to be larger than 2.5 mrad in the laboratory frame, when inverting direction of one of the tracks. This removes any remaining cosmic ray background and has negligible effect on signal. As an illustration, Fig. 5.10 shows the angle between two muon tracks for the 2010 CMS data on the left and signal MC simulation on the right. A few events remain in the data sample that appear to be cosmic candidates and are rejected by this cut. To isolate single muons from muons overlapping with jets, the sum of transverse momentum in the Tracker within a surrounding cone of $\Delta R \equiv \sqrt{(\Delta \eta)^{2}+(\Delta \phi)^{2}}<0.3$, is required to be less than $15 \%$ of the measured transverse momentum of the muon. The ECAL and HCAL are not used for muon isolation to reduce the effect from the photon FSR, to maintain more linear efficiency as function of dilepton mass, and to maximize the amount of good quality data.

The kinematic requirements in the laboratory frame are $|\eta| \leq 2.4$ and $p_{T}>18$ 


\section{CHAPTER 5. MEASUREMENT OF THE WEAK MIXING ANGLE}
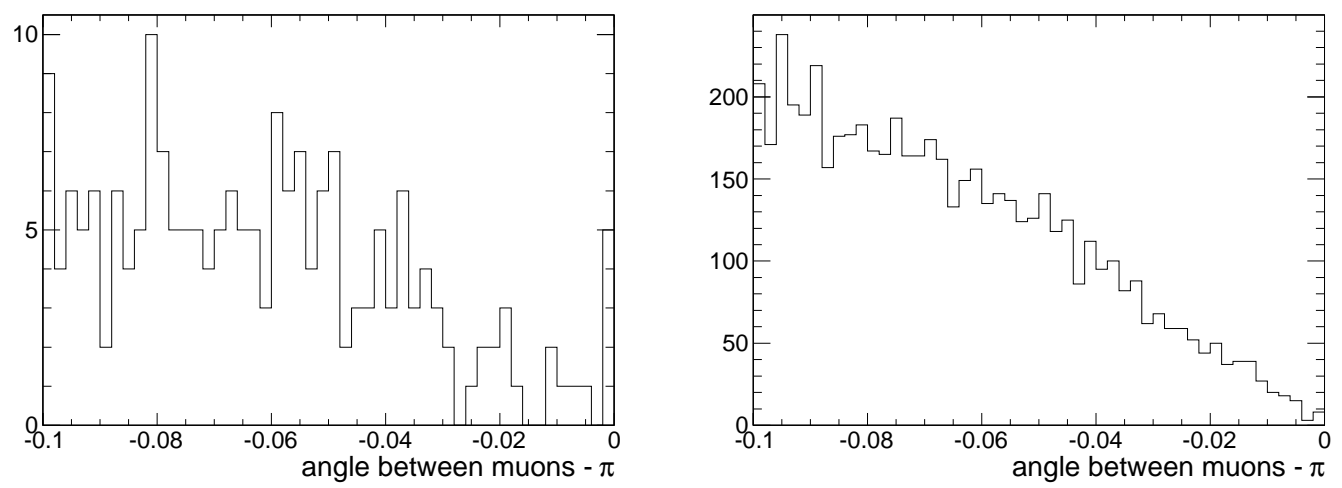

Figure 5.10: Distribution of the angle between the two muon tracks in data and signal $\mathrm{MC}$

and $8 \mathrm{GeV}$ for the two muons. However, we introduce additional requirements in the Collins-Soper frame in order to simplify the acceptance parameterization: $\left|\eta^{*}\right|<2.3$ and $p_{T}^{*}>18 \mathrm{GeV}$. The kinematic cuts in the laboratory frame are meant to ensure high quality events within the detector acceptance with good background suppression while the cuts in the Collins-Soper frame are implemented in the analytical parameterization. We design the kinematic cuts in such a way that selection in the lab frame is loose and does not cut into the the tighter selection in the Collins-Sopper frame. This is illustrated in Fig. 5.11, where the distribution of MC (POWHEG) events after progressive kinematic cuts are shown starting from all events selected by the trigger and track reconstruction (black), then after pseudo-rapidity cuts in the Collins-Soper frame (red) and laboratory frame (blue), and then after the transverse momentum cuts in the Collins-Soper frame (green) and laboratory frame (magenta). The overall acceptance efficiency of the kinematic cuts is high. We also require the dimuon transverse momentum to be less than $25 \mathrm{GeV}$ in order to suppress the contribution of events with 


\section{CHAPTER 5. MEASUREMENT OF THE WEAK MIXING ANGLE}

hard jet radiation which may cause a significant rotational inconsistency between the laboratory and Collins-Soper frame such that our kinematical assumptions break down.
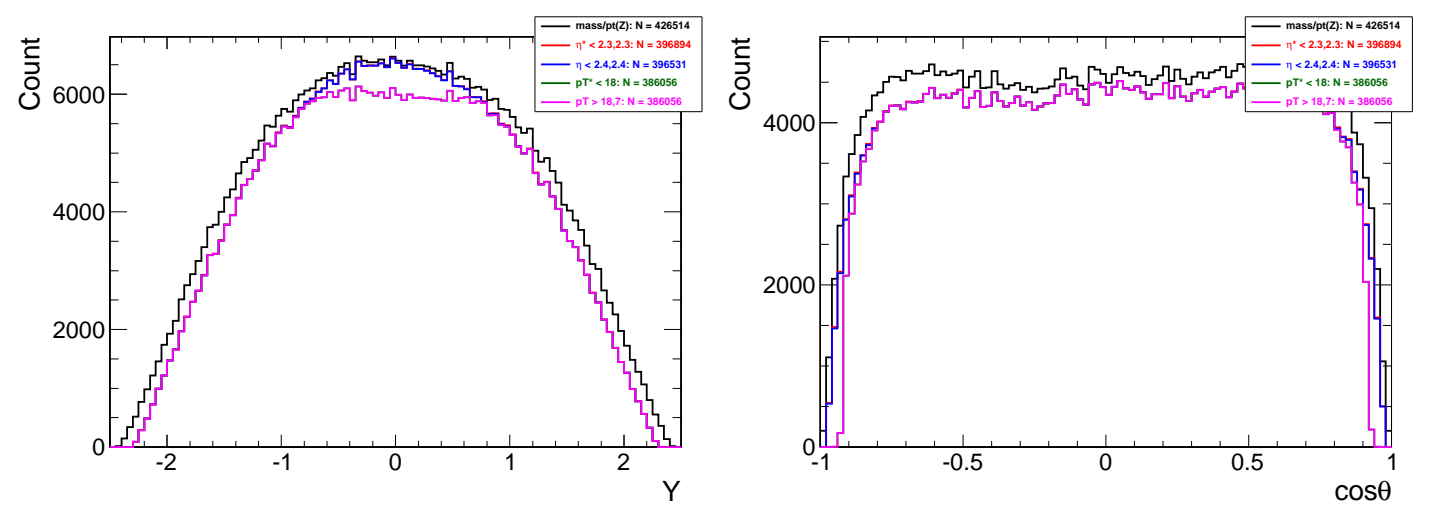

Figure 5.11: Distribution of MC (POWHEG) events after progressive kinematic cuts

Dilepton events are selected from events containing two oppositely charged, isolated, high $p_{T}$ muons with a dilepton invariant mass $m$ in the range $80-100 \mathrm{GeV}$. The dimuon system rapidity $Y$ is calculated from the lepton four-momenta as shown in Eq. (5.6). Restrictions on $\theta^{*}$ and $Y$ are motivated by detector acceptance effects which reduce their ranges, as we discuss below.

\subsection{Analysis Strategy}

We use an unbinned extended maximum-likelihood (ML) fit [70] that simultaneously describes the signal and background yields and the parameters of the $\left(Y, s, \cos \theta^{*}\right)$ 
distributions. The likelihood function is written as

$$
\mathcal{L}=\exp \left(-n_{\mathrm{sig}}-n_{\mathrm{bkg}}\right) \prod_{i}^{N}\left(n_{\mathrm{sig}} \times \mathcal{P}_{\mathrm{sig}}\left(\vec{x}_{i} ; \theta_{W} ; \vec{\xi}\right)+n_{\mathrm{bkg}} \times \mathcal{P}_{\mathrm{bkg}}\left(\vec{x}_{i} ; \vec{\xi}\right)\right)
$$

where each event candidate $i$ is characterized by a set of three observables $\vec{x}_{i}=$ $\left\{Y, s, \cos \theta^{*}\right\}_{i}, n_{\text {sig }}$ is the number of signal events, which includes all intermediate states $\left(\gamma^{*}, Z\right.$, and their interference), $n_{\mathrm{bkg}}$ is the small number of background events, $\mathcal{P}_{\text {type }}\left(\vec{x}_{i} ; \vec{\xi}\right)$ is the probability density function, and $\vec{\xi}$ are the parameters of that function. The number of selected events in the data is $N=297364$. The probability density function is defined for signal as

$$
\mathcal{P}_{\text {sig }}\left(Y, s, \cos \theta^{*} ; \theta_{W}\right)=\mathcal{G}\left(Y, s, \cos \theta^{*}\right) \times \int_{-\infty}^{+\infty} d x \mathcal{R}(x) \mathcal{P}_{\text {ideal }}\left(Y, s-x, \cos \theta^{*} ; \theta_{W}\right)
$$

The ideal angular distribution $\mathcal{P}_{\text {ideal }}\left(Y, s, \cos \theta^{*} ; \theta_{W}\right)$ in Eq. 5.15 is the $p p$ cross-section defined in Eq. 5.8. We correct $\mathcal{P}_{\text {ideal }}$ for detector effects, such as acceptance, resolution, and final state radiate (FSR).

\section{Detector Acceptance and Efficiency}

The acceptance function $\mathcal{G}\left(Y, s, \cos \theta^{*}\right)$ describes the non-uniform reconstruction efficiency across the phase-space of the three observables, which includes effects from online trigger requirements, detector acceptance, reconstruction algorithms, and selection requirements. It is defined in two parts: $\mathcal{G}\left(Y, s, \cos \theta^{*}\right)=\mathcal{G}_{1}\left(Y, s, \cos \theta^{*}\right) \times$ $\mathcal{G}_{2}\left(Y, s, \cos \theta^{*}\right)$. Described by $\mathcal{G}_{1}$, the most important effect is the loss of particles 


\section{CHAPTER 5. MEASUREMENT OF THE WEAK MIXING ANGLE}

along the beampipe while a secondary effect is the minimum transverse momentum requirement on the leptons. Otherwise the efficiency across the acceptance range is nearly uniform, defined by selection requirements $\left|\eta^{*}\right|<Y_{\max }=2.3$ and $p_{T}^{*}>p_{\min }=$ $18 \mathrm{GeV}$ in the previous section. The acceptance function $\mathcal{G}_{1}$ defines a sharp boundary in $\left(Y, s, \cos \theta^{*}\right)$ space, which can be expressed as limits on $\cos \theta^{*}$ for given $Y$ and $m$ values as follows

$$
\begin{aligned}
& \left|\cos \theta^{*}\right|<\tanh \left(Y_{\max }-|Y|\right), \\
& \left|\cos \theta^{*}\right|<\sqrt{1-\left(2 p_{\min } / m\right)^{2}},
\end{aligned}
$$

which is illustrated in Fig. 5.12 on the $\left(\cos \theta^{*}, Y\right)$ plane for a fixed value $m=m_{Z}$. The outer boundary corresponds to Eq. 5.16 and the horizontal lines correspond to Eq. 5.17.

Any non-uniformness in the acceptance region is described by $\mathcal{G}_{2}$ and may result from smooth transitions at the detector acceptance boundaries or non-uniform features of the track parameters in the phase space of the detector geometry. In Fig. 5.13, we illustrate the total efficiency of trigger, reconstruction, and acceptance of $Z \rightarrow \mu^{+} \mu^{-}$. In the top two plots, the total efficiency is given for the muon (antimuon) as a function of its pseudorapidity on the left (right). In the bottom three rows, we have the dilepton observables $Y, \cos \theta^{*}$, and $m$, respectively. In those plots, the left shows the efficiency as a function of those observables and the right has the raw comparison before and after trigger, reconstruction, and acceptance effects are been applied. The efficiency is close to uniform in the observables except near the acceptance boundaries in $\cos \theta^{*}$ 


\section{CHAPTER 5. MEASUREMENT OF THE WEAK MIXING ANGLE}

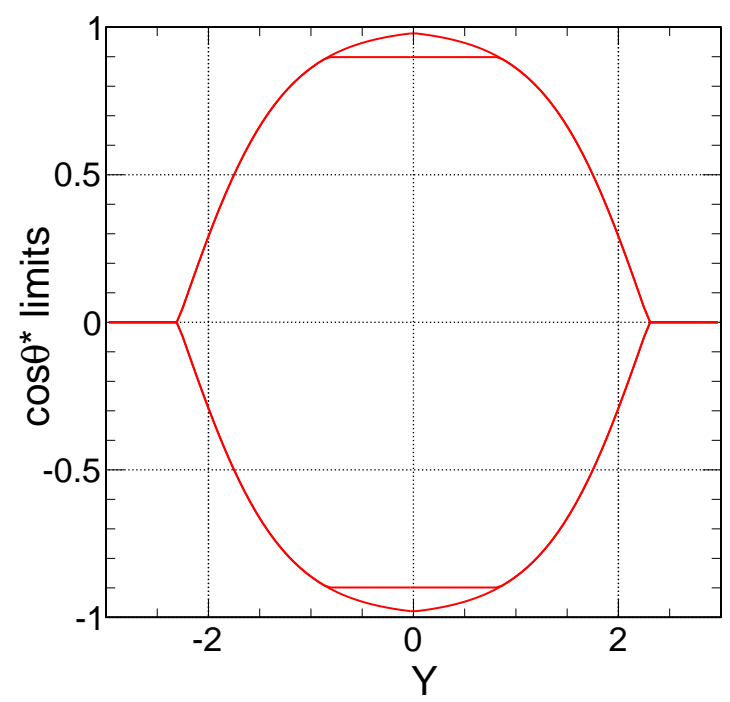

Figure 5.12: Accepted $\cos \theta^{*}$ range as a function of $Y$ for the kinematic selection in analysis

and $Y$. The efficiency is uniform in dilepton mass. In order to account for this effect, $\mathcal{G}_{2}$ is defined as a correlated function in $\cos \theta^{*}$ and $Y$ via even polynomials

$$
\mathcal{G}_{2}\left(Y, s, \cos \theta^{*}\right)=a_{1}+a_{2} Y^{2}+a_{3} Y^{8}+\left(c_{1}+c_{2} Y^{2}+c_{3} Y^{8}\right) \cos ^{8} \theta^{*}
$$

where $a_{1}, a_{2}, a_{3}, c_{1}, c_{2}, c_{3}$ are free parameters. The efficiency function is described with only even polynomials since it is symmetric due to the proton-proton symmetry; the direction of the boost is random, equally probable to be forward or backward, in the laboratory frame, thus asymmetric detector effects are washed out. We fit for the free parameters in Eq. 5.18 using the POWHEG and CMS detector simulation. The visualization of $\mathcal{G}_{2}$ is given in Fig. 5.14. We look at this efficiency effect and the agreement between simulation and data in greater detail in Section 5.4 on systematic uncertainties and find it has a very weak effect on $\sin ^{2} \theta_{\text {eff. }}$ 


\section{CHAPTER 5. MEASUREMENT OF THE WEAK MIXING ANGLE}
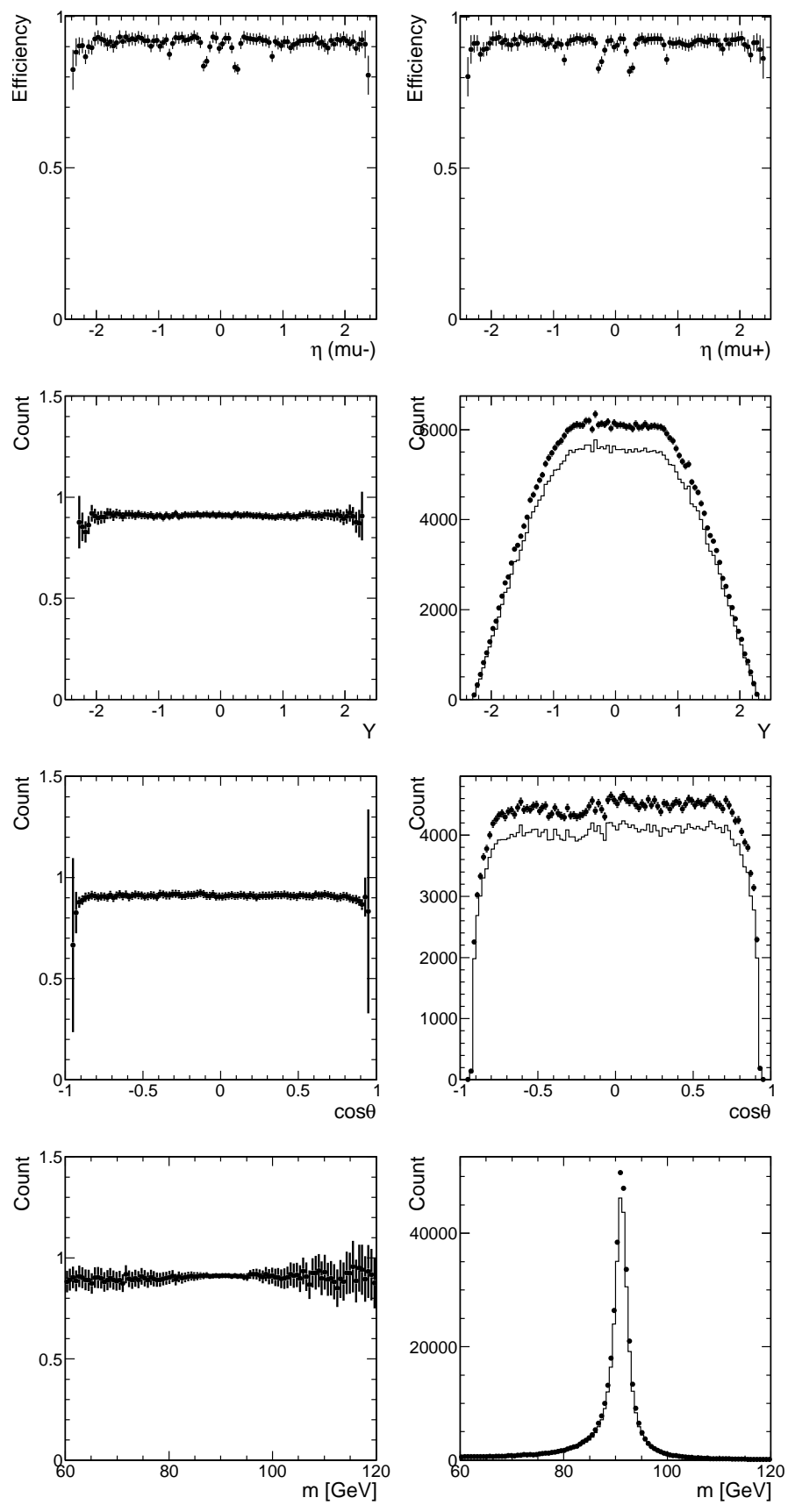

Figure 5.13: Total efficiency of trigger, reconstruction, and acceptance as a function of muon $\eta$, dilepton $Y, \cos \theta^{*}$, and $m$. 


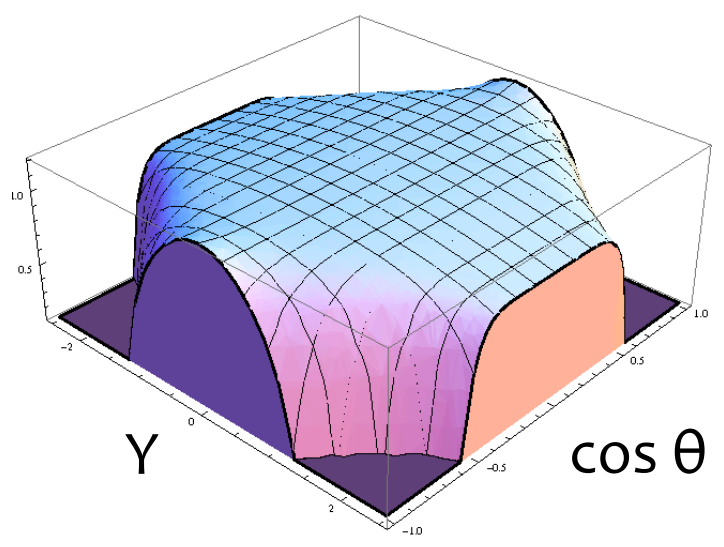

Figure 5.14: The efficiency function $\mathcal{G}_{2}$ fitted from using simulation

\section{Detector Resolution and FSR}

The effect of the smearing of the muon track parameters, such as muon momentum and direction, due to detector resolution and FSR is most evident in the invariant mass distribution. This effect is parameterized with the function $\mathcal{R}(x)$ in Eq. 5.15 where $\mathcal{R}(x)$ is convoluted with ideal description of the Drell-Yan process. The form of $\mathcal{R}(x)$ is a quadruple Gaussian function in order to fully describe the FSR tail and allow for analytical convolution.

Both acceptance and resolution effects are illustrated in Fig. 5.15, where analytical parameterization of $Y, m$, and $\cos \theta^{*}$ is in good agreement with LO simulation in both QCD and EWK, as generated by PYTHIA. In this illustration photon FSR is included and the major detector effects are introduced in the following way. For the three track parameters $\left(p_{T}, \phi, \theta\right)$, we apply Gaussian random smearing with an rms 
$\Delta p_{T}=0.025 \times p_{T}+0.0001 \times p_{T}^{2}(\mathrm{GeV}), \Delta \phi=\Delta \theta=0.001(\mathrm{rad})$, and neglect resolution effects on the track origin. We find this simplified simulation of detector effects useful to isolate production model uncertainties from detector effect parameterization.

Further studies are performed with full GEANT-based modeling of the CMS detector using POWHEG simulation of the dimuon events and with PYTHIA simulation of the parton showering and FSR. In the parameterization of the resolution function $\mathcal{R}(x)$, the FSR is modeled with PYTHIA and resolution effects are taken from the full CMS detector simulation, including effects of Tracker alignment on track parameter resolution. The resolution function $\mathcal{R}(x)$ is extracted from the realistic simulation POWHEG and CMS detector simulation and is used on the final fit to the data. Systematic effects on the resolution function from alignment and FSR are explored in greater detail in Section 5.4.

\section{Background parameterization}

The background contributions in the $q \bar{q} \rightarrow \gamma^{*} / Z \rightarrow \mu^{-} \mu^{+}$channel are small compared to the signal yield. The background consists of the cross-feed from the $q \bar{q} \rightarrow Z / \gamma^{*} \rightarrow \tau^{+} \tau^{-}$process, $\mathrm{QCD}, t \bar{t}$, and di-boson production $(W W, W Z, Z Z)$ in nearly equal contributions. The yields are measured with $M C$ simulation and in the case of QCD are cross-checked with data-driven techniques. The simulation for the other EWK background contributions are considered relatively stable considering the amount of background in this channel. The expected background yield is approxi- 


\section{CHAPTER 5. MEASUREMENT OF THE WEAK MIXING ANGLE}
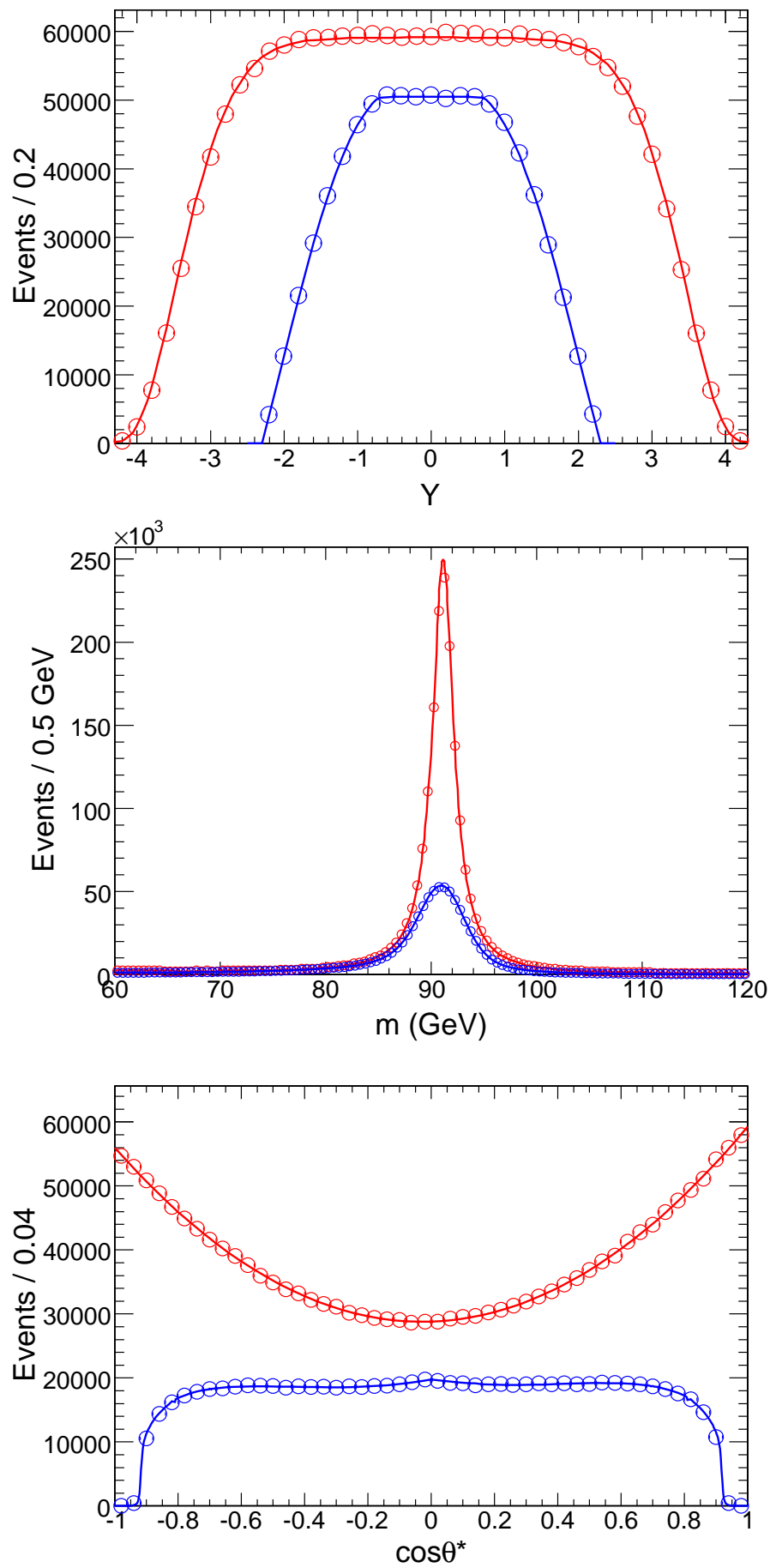

Figure 5.15: Distribution of $Y, m, \cos \theta^{*}$ for the analytical model and PYTHIA simulation before and after detector acceptance, efficiency, and resolution and FSR effects. 

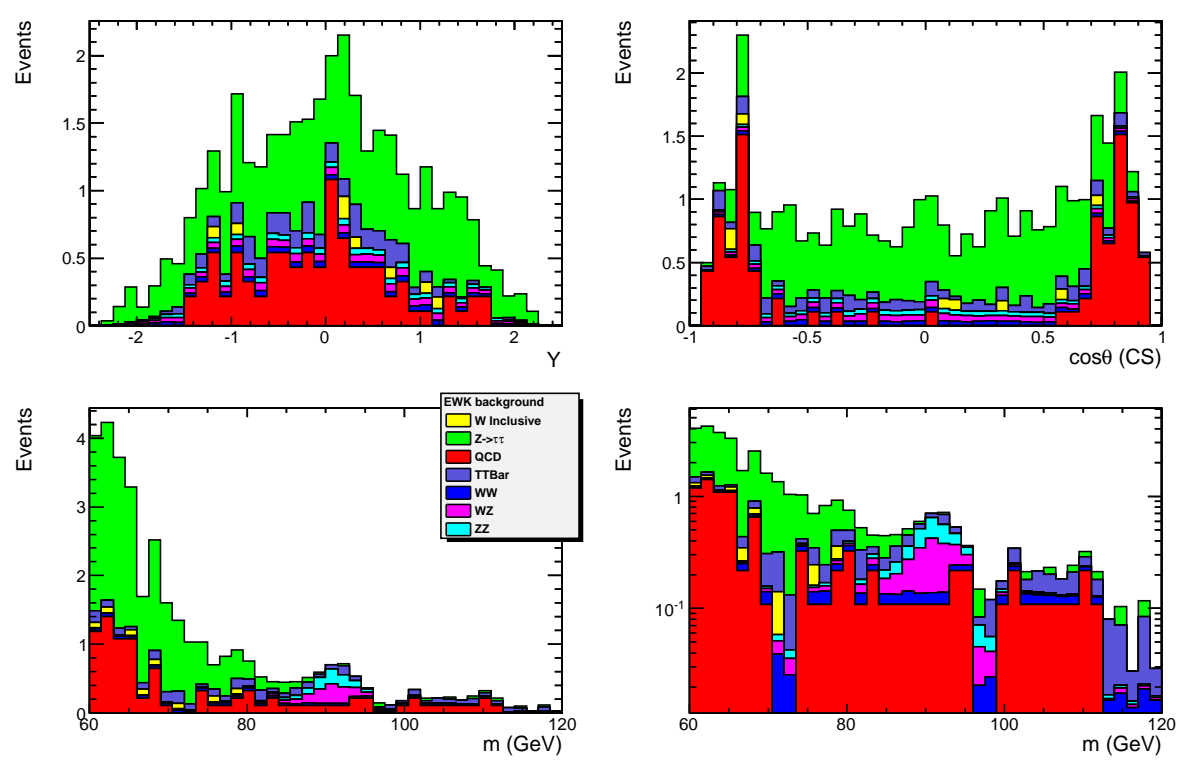

Figure 5.16: Distributions of the backgrounds to the Drell-Yan process

mately $0.05 \%$ of the signal yield and thus even large uncertainties in the background yields do not affect the measurement of $\sin ^{2} \theta_{\text {eff }}$ very much. A check of the systematic effect due to background contributions is performed in Section 5.4. In Fig. 5.16 the background distributions for each contribution are shown in the observables $Y, \cos \theta^{*}$ and $m$. The mass range from $60-120 \mathrm{GeV}$ is illustrated in the figure to show that a non-trivial background contribution exists in the region from $60-80 \mathrm{GeV}$ while the fit in the mass range $80-100 \mathrm{GeV}$ is relatively background-free. The shapes of these distributions are fitted and used in the full likelihood model given in Eq. 5.14. This parameterization is required to provide the most accurate comparison of the likelihood test described in Section 5.5. 


\subsection{Systematic Uncertainties}

While a blind analysis of the data has been performed where the central value of the final fit is not revealed until the analysis is fully reviewed, fits on the data with the given statistics for this analysis yield a statistical error on $\sin ^{2} \theta_{W}$ of approximately $1 \%$ or 0.002 . The list of systematic uncertainties on the measurement of $\sin ^{2} \theta_{\text {eff }}$ and associated corrections to the fit values is shown in Table 5.2. These uncertainties arise from both theoretical assumptions and detector modeling. Each contribution is discussed below in more detail. In comparing our overall systematic error with the expected statistical error, we find that they are comparable.

Table 5.2: Systematic uncertainties and corrections to the fit values in the measurement of $\sin ^{2} \theta_{\text {eff. }}$.

\begin{tabular}{|c|c|c|}
\hline \hline source & correction & uncertainty \\
\hline LO model (EWK) & - & \pm 0.0002 \\
PDF & - & \pm 0.0013 \\
FSR & - & \pm 0.0011 \\
LO model (QCD) & +0.0012 & \pm 0.0012 \\
resolution and alignment & +0.0007 & \pm 0.0013 \\
efficiency and acceptance & - & \pm 0.0003 \\
background & - & \pm 0.0001 \\
\hline total & +0.0019 & \pm 0.0025 \\
\hline
\end{tabular}




\section{LO model (EWK)}

Effects from NLO EWK contributions are expected to be small compared to precision in this analysis. Calculations with the ZFITTER program [71] in the on-shell scheme for $m_{t}=175 \mathrm{GeV}$ and $m_{H}=120 \mathrm{GeV}$ indicate that the variation of the effective value of $\sin ^{2} \theta_{\text {eff }}$ for light quarks is within 0.0002 of the lepton values. See Table 5.3 for the summary of results. It is only the heavier $b$ quark which requires corrections of the order 0.001 , but given that only about $2.5 \%$ of di-leptons are produced in $b \bar{b}$ interactions and no angular asymmetry can be measured with this initial state, this has negligible effect on the current measurements.

Table 5.3: Effective values of $\sin ^{2} \theta_{\text {eff }}$ for different fermion couplings to the $Z$ boson.

\begin{tabular}{ll}
\hline \hline source & uncertainty \\
\hline $\mathrm{e}$ & 0.23152 \\
$\mu$ & 0.23152 \\
$\tau$ & 0.23152 \\
up & 0.23142 \\
down & 0.23129 \\
charm & 0.23142 \\
strange & 0.23129 \\
bottom & 0.23294 \\
\hline \hline
\end{tabular}


CHAPTER 5. MEASUREMENT OF THE WEAK MIXING ANGLE

\section{PDF uncertainties}

Systematic effects associated with the uncertainties from parton distribution functions are detailed. By looking at the variations of our fit value of $\sin ^{2} \theta_{W}$ using POWHEG simulation as a function of the variations internal to a single PDF set or between different PDF sets, we can quantify the systematic uncertainty coming from PDFs. The official procedure is given by the PDF4LHC working group in [72] and [73].

In Fig. 5.17, the variations of $\sin ^{2} \theta_{W}$ are shown as a function of the internal degrees of freedom of the PDFs. We re-weight our POWHEG sample based on the LHAPDF [74] prescription for the CT10 [55], MSTW [56], and NNPDF [57] PDF sets. In Fig. 5.17, the variations as a function of the CT10 eigenvectors (top left) and $\alpha_{s}$ (top right) as well as for the MSTW eigenvectors (bottom left) and NNPDF replicas (bottom right) are shown. The calculation of the PDF and $\alpha_{s}$ uncertainties are given in Eqs. 3 and 9 of [72], respectively, for Hessian PDF sets (CTEQ and MSTW). Then, the two are added in quadrature to get the full PDF $+\alpha_{s}$ uncertainties. To give an example of the calculation, we give Eq. 3 from [72] here:

$$
\begin{aligned}
\Delta X_{\max }^{+} & =\sqrt{\sum_{i=1}^{N}\left[\max \left(X_{i}^{+}-X_{0}, X_{i}^{-}-X_{0}, 0\right)\right]^{2}} \\
\Delta X_{\max }^{-} & =\sqrt{\sum_{i=1}^{N}\left[\max \left(X_{0}-X_{i}^{+}, X_{0}-X_{i}^{-}, 0\right)\right]^{2}}
\end{aligned}
$$

The $\alpha_{s}$ uncertainties are neglible in the given context. Eqs. (5) and (6) of [72] give the uncertainties for the NNPDF set.

For each contributing PDF sets, we extract the central value, $\left\langle\sin ^{2} \theta_{W}\right\rangle$, and also 
CHAPTER 5. MEASUREMENT OF THE WEAK MIXING ANGLE
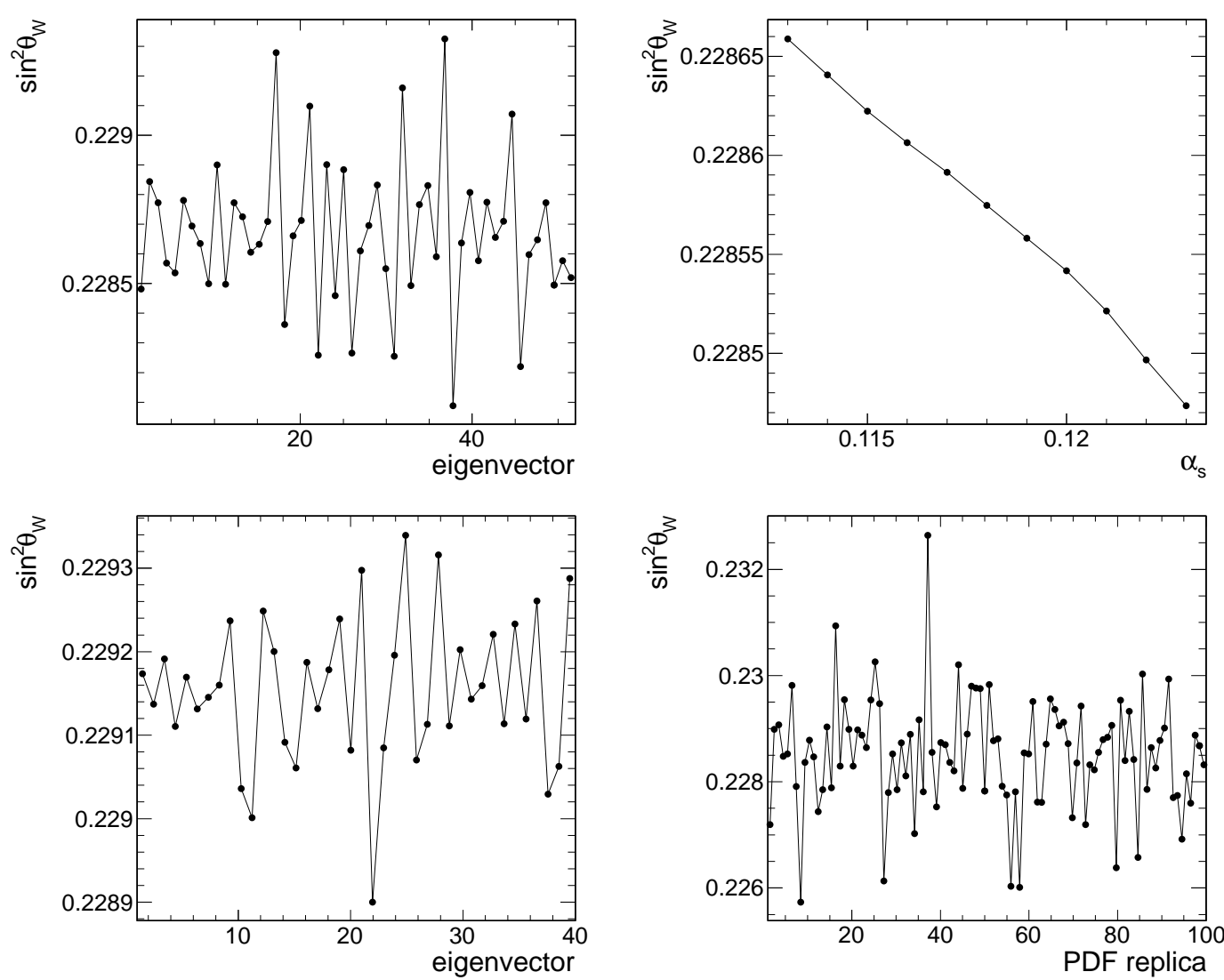

Figure 5.17: Effect on $\sin ^{2} \theta_{W}$ of PDF variations for various PDF models.

the systematic uncertainties, $\Delta_{+}$and $\Delta_{-}$. These are quoted as corrections to the nominal CT10 result and the corresponding upper and lower errors:

- CT10: $\Delta \sin ^{2} \theta_{W}=0.00000_{-0.00121}^{+0.00130}$

- MSTW2008: $\Delta \sin ^{2} \theta_{W}=+0.00050_{-0.00041}^{+0.00040}$

- NNPDF21: $\Delta \sin ^{2} \theta_{W}=+0.00030_{-0.00073}^{+0.00073}$

In order to combine uncertainties from all the PDF sets, an envelope is taken of all the contributing PDF sets and the error is extracted by taking the midpoint value of the 


\section{CHAPTER 5. MEASUREMENT OF THE WEAK MIXING ANGLE}

envelope and calling the uncertainty the distance to the edge of the envelope. We find that the main systematic uncertainties come from the CT10 PDF set and they cover the range of both NNPDF21 and MSTW2008. The total PDF systematic uncertainty from this procedure on $\sin ^{2} \theta_{W}$ is calculated to be, $\Delta_{P D F}=0.0013$.

\section{FSR}

In the parameterization of the resolution function $\mathcal{R}(x)$, the FSR is modeled with PYTHIA and we use alternative models with HORACE [75] and PHOTOS [76] for crosschecks. We use stand-alone generation without detailed CMS detector simulation for these studies. We first fit samples with FSR from Pythia, HORACE $\alpha$, HORACE "exp," and PHOTOS for parameters of the resolution function modeling the effect of the FSR. The three generators Pythia, HORACE $\alpha$, and PHOTOS are calculations with parton showering while HORACE exp is a state-of-the-art computation that includes additional higher order corrections.

All three generator programs provide similar results within about 0.0010 with $O(\alpha)$ calculations. We find that the typical deviations of the fitted $\sin ^{2} \theta_{\text {eff }}$ value are within 0.0010 of the original value if an alternative FSR model is used in the resolution function. In Fig. 5.18, we show the differences between the different FSR models in the tail of the $Z$ mass distribution. The results of various fits are summarized in Table 5.4. The relevant comparisons are between the same sample fit with different resolution functions (horizontal rows). We assign a systematic error of 0.0011 based 
on the difference between Pythia (used in our simulation) and HORACE(exp), which is the state-of-the-art EWK calculation.

Table 5.4: Results of the MC cross-fits using Horace $(\alpha)$, Horace (exp), Pythia (Pythia FSR), and Pythia (PHOTOS FSR).

\begin{tabular}{|r||c|c|c|c|c|}
\hline Resolution function used: & Horace $(\alpha)$ & Horace $(\exp )$ & Pythia & PHOTOS & error size \\
\hline \hline on Horace $(\alpha)$ sample (10M) & 0.2298 & 0.2295 & 0.2284 & 0.2296 & $\sim 0.0007$ \\
\hline on Horace $(\exp )$ sample (10M) & 0.2309 & 0.2307 & 0.2295 & 0.2308 & $\sim 0.0007$ \\
\hline on Pythia sample (9M) & 0.2322 & 0.2319 & 0.2308 & 0.2321 & $\sim 0.0006$ \\
\hline on PHOTOS sample (10M) & 0.2309 & 0.2306 & 0.2295 & 0.2314 & $\sim 0.0006$ \\
\hline
\end{tabular}

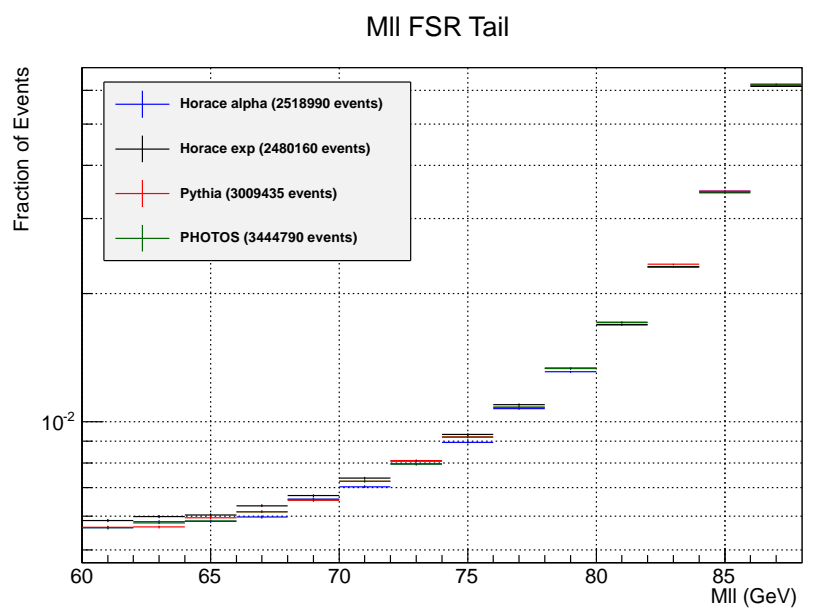

Figure 5.18: FSR tail in the range $60<m<90 \mathrm{GeV}$ in the process $q \bar{q} \rightarrow \gamma^{*} / Z \rightarrow$ $\mu^{-} \mu^{+}$generated with four different FSR models

We also come to a similar conclusion when we re-weight the fraction of FSR radiation in simulation. This re-weighting technique is based on the comparison of FSR radiation fraction between data and PYTHIA simulation. Table 5.5 shows variation of $\sin ^{2} \theta_{\text {eff }}$ in $\mathrm{MC}$ simulation with two re-weighting factors. In one case the fraction of events when the di-lepton mass changes by more than $1 \mathrm{GeV}$ due to 
FSR is reduced or increased by $2 \%$. In the other case the fractional change is $3 \%$. In Table 5.5, we present the systematic effect from FSR modeling in different mass ranges. The table shows the increase in the systematic uncertainties from FSR as the fit mass range increases and the motivation for fitting in the $80-120 \mathrm{GeV}$ range.

Table 5.5: Results of the FSR re-weighting and the change of $\sin ^{2} \theta_{\text {eff }}$.

\begin{tabular}{|c|c|c|c|c|}
\hline weight & $60-120 \mathrm{GeV}$ & $70-110 \mathrm{GeV}$ & $75-105 \mathrm{GeV}$ & $80-100 \mathrm{GeV}$ \\
\hline$\Delta 2 \%$ & 0.0012 & 0.0012 & 0.0009 & 0.0005 \\
\hline$\Delta 3 \%$ & 0.0018 & 0.0018 & 0.0013 & 0.0008 \\
\hline
\end{tabular}

\section{Tracker alignment systematics}

The detector resolution effects in muon track reconstruction are dominated by effects of the Silicon Tracker alignment. The procedure of tracker alignment was discussed in more detail in Section 3.3. We perform a realistic simulation of the alignment procedure to model statistical precision in track reconstruction. This is the so-called "Realistic" scenario of the tracker geometry, which is obtained with a similar alignment procedure as used for the data. We also consider systematic effects coming from the $\chi^{2}$-invariant deformations of the tracker (weak modes) by studying nine basic distortions in the cylindrical system. The size of these weak modes in the data are studied by observing the dependency of the dilepton invariant mass distribution on 


\section{CHAPTER 5. MEASUREMENT OF THE WEAK MIXING ANGLE}

the observables, which should be invariant, in the phase space of the detector.

In order to study the effect of misalignment, we vary our simulation geometry and extract a fit result for $\sin ^{2} \theta_{\text {eff }}$ in each case where our model remains the same and is tuned to the baseline simulation geometry. The baseline geometry in simulation is "Realistic" alignment. We then use the model tuned for "Realistic" and extract fits to simulation which has been re-reconstructed with 10 other alignment geometries: the design geometry and "Realistic" +9 weak modes. The weak modes represent $\chi^{2}$-invariant deformations of the tracker and are called Bowing, Curl, Elliptical, Radial, Sagitta, Skew, Telescope, Twist, and Z-expansion. These misalignment scenarios have been created by introducing the corresponding coherent distortion in the Tracker geometry and then repeating the procedure of alignment to observe the introduced distortion to be taken out, fully or partially. The remaining distortion was taken as a misalignment scenario. The typical initial distortions were taken to be of the size $200 \mu \mathrm{m}$, which is the typical size of constraints from detector survey, placement tolerance, and the observed agreement in the alignment procedure, such as comparing independent alignment algorithms.

The fits of these nine weak modes are performed. The typical errors in the fits are 0.0006 , but due to close to $100 \%$ overlap of events, the error on the difference is much smaller. In each case, we have our re-reconstructed POWHEG simulation and we generate toy experiments and extract the value of $\sin ^{2} \theta_{\text {eff }}$ by fitting in each toy experiment. The fit result is the mean result of all toy experiments. All variations stay 


\section{CHAPTER 5. MEASUREMENT OF THE WEAK MIXING ANGLE}

within \pm 0.0009 and in general exhibit similar features.

In addition, we characterize the effect of the weak modes by looking at the dilepton invariant mass as a function of our observable $\cos \theta^{*}$. The fit value using the design geometry, which has no alignment distortions, allows us to isolate any systematic effects from alignment. We subtract the dilepton mass versus $\cos \theta^{*}$ distribution for the design geometry from the "Realistic" and "Realistic + weak modes" scenarios to determine any remaining residual bias. In particular we highlight the "Realistic + Curl" mode which from initial studies we found to cause a large bias in the fit of

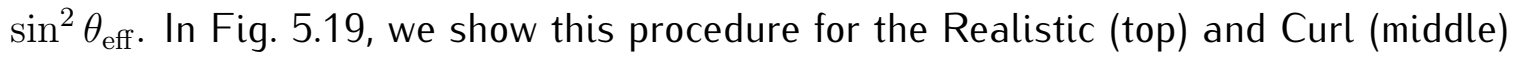
scenarios. The bottom of Fig. 5.19 shows the sample plot for the CMS data sample where the overall shift of data versus $M C$ is explained by the difference in the overall energy scale, which we also float in the fit to data as discussed below. In all plots on the left the black points show Ideal MC scenario which is compared the the scenarios in red. The subtraction and linear fit is shown on the right. In Table 5.6, the slope fit values are shown for the cases in Fig. 5.19. From this table, we quantify the bias from the Realistic and Curl scenarios, but it also gives us confidence that the bias is not as significant in the data. A similar procedure is followed for the other weak modes. Similar limits on the bias are found.

The full fit results with the "Realistic" and design geometries are

- Realistic: $\sin ^{2} \theta_{W}=0.2286 \pm 0.0006$

- Design: $\sin ^{2} \theta_{W}=0.2299 \pm 0.0006$ 
CHAPTER 5. MEASUREMENT OF THE WEAK MIXING ANGLE
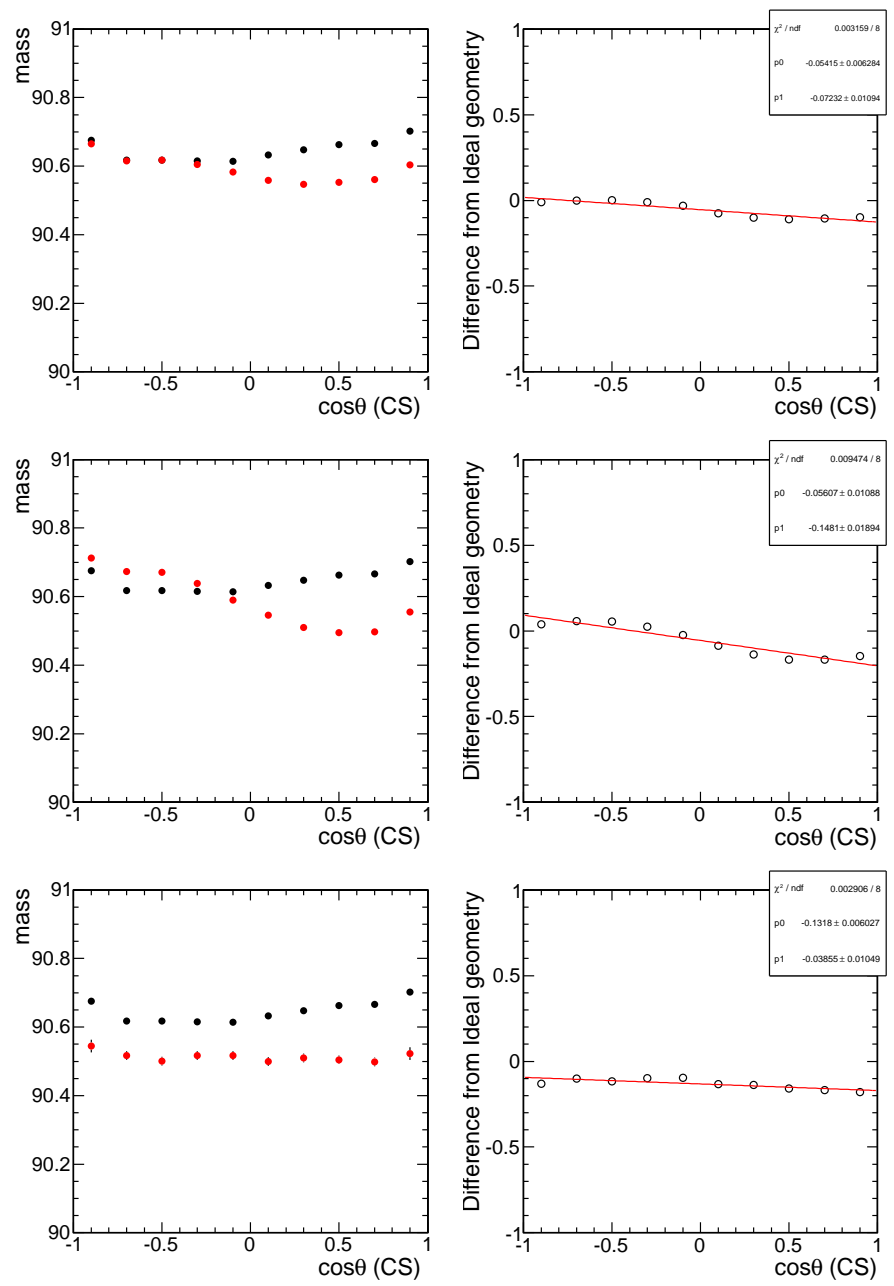

Figure 5.19: Profile of the di-lepton invariant mass distribution as a function of $\cos \theta^{*}$.

Table 5.6: Slope of the profile plots shown in Fig. 5.19

\begin{tabular}{|c|c|}
\hline Alignment scenario & slope $[\mathrm{GeV}]$ \\
\hline Realistic & -0.072 \\
Curl & -0.148 \\
data & -0.039 \\
\hline
\end{tabular}




\section{CHAPTER 5. MEASUREMENT OF THE WEAK MIXING ANGLE}

We should note that it is critical to redo the resolution function of the Ideal geometry since its resolution is much better. This is shown in Fig. 5.20 where the points show distribution with design alignment geometry in reconstruction, the blue line show the probability distribution with the self-consistent resolution function, and the red line shows the probability distribution with Realistic resolution function. From the fit with the design geometry, there is also a remaining effect of the order of 0.0010 even with Ideal geometry and these effects are consistent with those discussed in Section 5.4.

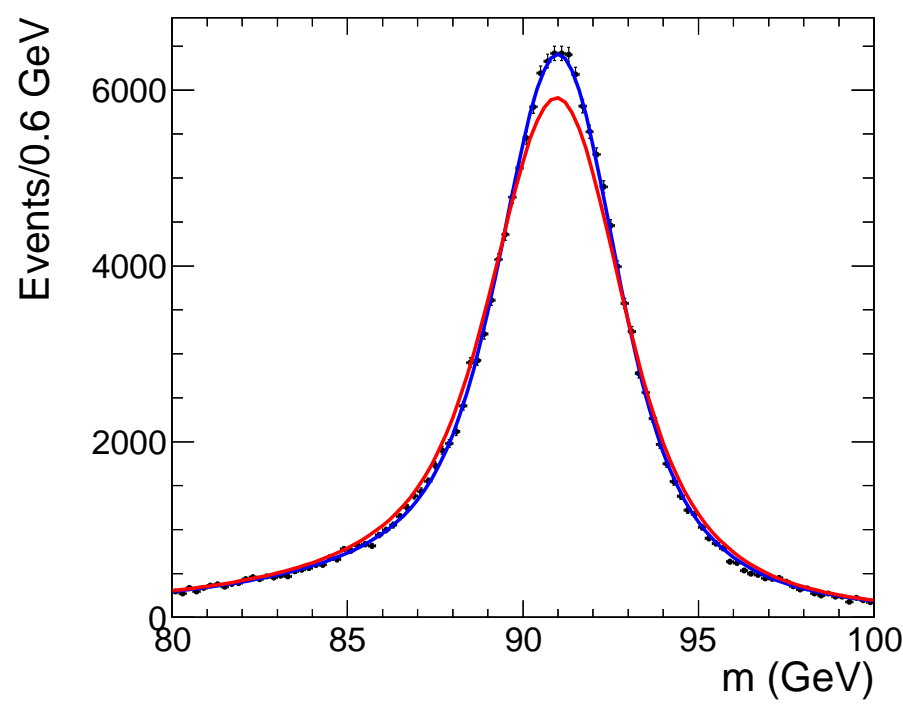

Figure 5.20: Invariant mass of the di-lepton pair in simulation for the design and Realistic geometries

Based on the above studies, we apply corrections to the fit results in data and MC and assign the corresponding systematic uncertainties due to alignment effects. These corrections are:

- data: $+0.0007 \pm 0.0013$ 
- Realistic MC: $+0.0013 \pm 0.0013$

. The correction in MC is based on the difference observed between Ideal and Realistic scenarios. The correction in data is based on the fact that the bias in data is expected to be twice smaller, as illustrated in Fig. 5.19 and Table 5.6.

In order to minimize uncertainties from the energy scale bias in track reconstruction, the reconstructed $Z$ mass is left free in the fit, effectively allowing the energy scale to be determined from the fit to data. We observe an effective shift of the energy scale of approximately $100 \mathrm{MeV}$ which is reflected if Fig. 5.19. We also allow the resolution function parameters to be free in the fit as a cross-check of both detector resolution and FSR effects and find results consistent with the nominal fit.

\section{LO model (QCD)}

In the analysis we apply the requirement that the di-muon transverse momentum $p_{T}(Z)$ be less than $25 \mathrm{GeV}$. This suppresses the contribution of events with hard jet radiation and makes kinematics similar to the LO model. However, to study the residual effects of modeling both initial state and PDFs at LO, we perform the following studies of the $p_{T}(Z)$ requirement. We perform a test with MC samples using POWHEG and CT10 PDFs and the full CMSSW simulation. Differences of the fitted $\sin ^{2} \theta_{\text {eff }}$ value in four different $p_{T}(Z)$ ranges compared to the nominal selection $p_{T}(Z)<25 \mathrm{GeV}$ are shown in Table 5.7. The overall stability of results of the order of 0.001 is observed. The $p_{T}(Z)$ is not perfectly reproduced in MC. In Fig. 5.21 we compare measured 


\section{CHAPTER 5. MEASUREMENT OF THE WEAK MIXING ANGLE}

$p_{T}$ spectra in data (black) and $\mathrm{MC}$ (red) on the left. We re-weight the MC events according to observed data/MC difference to match the $p_{T}$ spectra on the left and observe 0.0004 change in the $\sin ^{2} \theta_{\text {eff }}$ fitted value.

Table 5.7: Differences of the fitted $\sin ^{2} \theta_{\text {eff }}$ value in four different $p_{T}(Z)$ ranges for data and simulation

\begin{tabular}{|c|c|c|}
\hline & $\mathrm{MC}$ & data \\
\hline$p_{T}<15$ & -0.0013 & $+0.0001 \pm 0.0010$ \\
$p_{T}<20$ & -0.0007 & $-0.0008 \pm 0.0006$ \\
$p_{T}<25$ & - & - \\
$p_{T}<30$ & +0.0004 & $+0.0000 \pm 0.0004$ \\
$p_{T}<50$ & +0.0007 & $-0.0007 \pm 0.0008$ \\
\hline
\end{tabular}
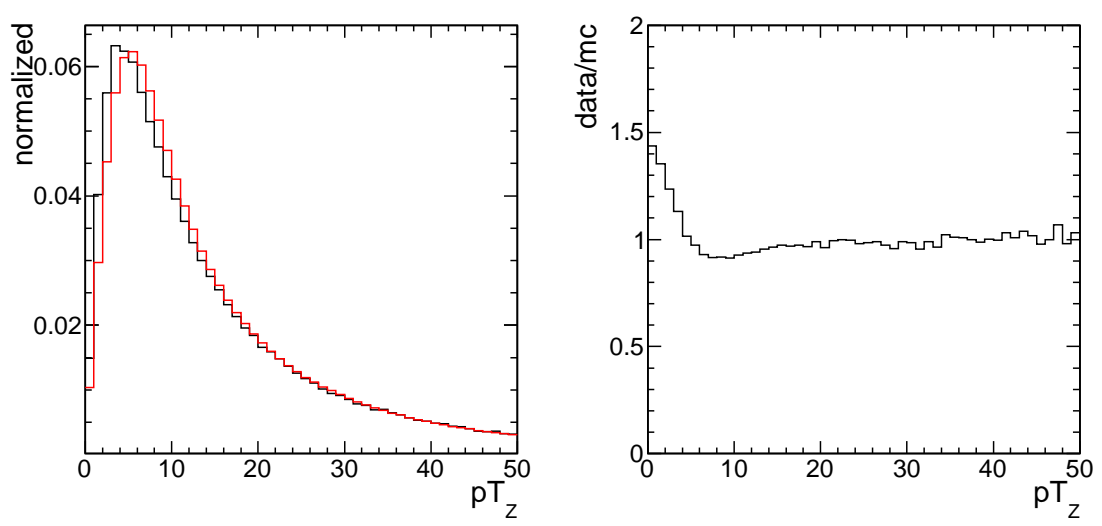

Figure 5.21: $p_{T}$ distribution of the di-muon system in data and simulation and the corresponding ratio

Based on the above studies, we apply a correction to the fit results in data and MC and assign the corresponding systematic uncertainties due to NLO effects. These 
corrections are summarized in Table 5.8. This correction is consistent with zero within $2 \sigma$ of precision in the above tests. However, we believe that the overall uncertainty of the level \pm 0.0012 is representative of the typical effects associated with the LO model and $p_{T}$ effects. It may in fact include double-counting of the PDF uncertainties which are of the same order and could affect these tests due to LO model of the PDFs as well.

Table 5.8: Correction to the $\sin ^{2} \theta_{\text {eff }}$ fit result in data and MC due to NLO (QCD) effects.

\begin{tabular}{|c|c|}
\hline & $\Delta \sin ^{2} \theta_{\text {eff }}$ \\
\hline data & $+0.0012 \pm 0.0012$ \\
\hline Realistic MC & $+0.0012 \pm 0.0012$ \\
\hline
\end{tabular}

\section{Efficiency and acceptance}

We find very weak sensitivity to details of efficiency parameterization $\mathcal{G}\left(Y, s, \cos \theta^{*}\right)$ across the acceptance range because only even terms in $\cos \theta^{*}$ and $Y$ contribute to efficiency model. This leads to negligible effects on the odd terms in the angular distribution which are sensitive to $\sin ^{2} \theta_{\text {eff. }}$. The sign of $Y$ is defined by the di-muon system direction along the beamline and has no preferential direction. The sign of $\cos \theta^{*}$ is defined by the charge of the "forward" lepton. Therefore the cylindrical symmetry combined with the random nature of the "forward" direction leads to the symmetry in the 


\section{CHAPTER 5. MEASUREMENT OF THE WEAK MIXING ANGLE}

efficiency function. This assessment has been verified with detailed GEANT-based simulation of the CMS detector, including major calibration and alignment effects. Even the extreme variation of the efficiency model $\mathcal{G}\left(Y, s, \cos \theta^{*}\right)$ to being flat across the acceptance range leads to negligible changes in the fit results with simulated samples. We also allow parameters of the model to be free in the fit to data. We assign a systematic error of 0.0003 due to efficiency and acceptance parameterization, which is the level of consistency of results in these studies.

\section{Background}

The number of background events $n_{\mathrm{bkg}}$ is fixed to the expected value of 157 events and is varied according to its associated uncertainties. The relative size of the background EWK processes is expected to be reproduced well by simulation, while we assign a 50\% uncertainty to the QCD rate based on studies with wrong-sign lepton pairs. However, in the mass range $80<m<100 \mathrm{GeV}$ the fraction of background is only $0.05 \%$ and the fit results do not depend on exact treatment of background. The measured $\sin ^{2} \theta_{\text {eff }}$ value remains stable within 0.0001 even when background is removed from the model. 


\section{Summary of systematics}

In the end we add the following correction and systematic uncertainty to the result:

$$
\sin ^{2} \theta_{\text {eff }}=+0.0019 \pm 0.0025,
$$

The correction is based on observed alignment and NLO (QCD) effects given above and the systematic uncertainty is the sum in quadrature of all effects in Table 5.2.

\section{Further Cross-checks}

We test the performance of the fitting procedure with generated samples, each with the observed number of events. Signal events are used from POWHEG-based CMS detector simulation with an input value of $\sin ^{2} \theta_{\text {eff }}=0.2311$. The number of background events is Poisson-distributed according to expectation. After the corrections discussed above are applied, the distribution $\left(\sin ^{2} \theta_{\text {eff }}-0.2311\right)$ normalized by the error is in agreement with a unit-width Gaussian distribution centered at zero, confirming that the error estimates are correct. A comparison of the $M C$ sample projections and the probability density functions are shown in Fig. 5.22. We have also repeated these studies with pure generated experiments, where the three observables were generated according to the likelihood model, and find consistent results.

While analysis of the data is performed blind, we examine the quality of the fit on data by comparing the data distributions to the likelihood model expectation and comparing the fit likelihood $\mathcal{L}$ value and observed statistical error to those expected 
CHAPTER 5. MEASUREMENT OF THE WEAK MIXING ANGLE

with generated samples. Projections of the data and the probability density functions are shown in Fig. 5.23 and exhibit similar agreement between the data and the model as with simulation shown in Fig. 5.22. Correction for the energy scale is already included in the fit model as discussed above. The observed statistical error is in good agreement with expectation from the generated experiments discussed above. The value of the likelihood $\mathcal{L}$ is also in agreement with expectations, exhibiting a similar difference from the pure generated experiments as observed for POWHEG-based CMS detector simulation. The level of the difference is consistent with the typical differences due to not perfect efficiency function modeling and NLO effects which do not affect the result of analysis within systematic uncertainties assigned. For example, the $-\log \mathcal{L}=-2,145,050$ in data, the $-\log \mathcal{L}=-2,145,660 \pm 113$ in $\mathrm{MC}$ and the $-\log \mathcal{L}=-2,146,580 \pm 99$ in pure generated experiments. An improvement of order of $\Delta \log \mathcal{L}=100$ happens just from floating the efficiency model parameters in data, with essentially no change in results.

\subsection{Results and Discussion}

The result of the analysis after unblinding the fit, which includes systematic uncertainties and corrections from Table 5.2, is

$$
\sin ^{2} \theta_{\text {eff }}=0.2287 \pm 0.0020 \text { (stat.) } \pm 0.0025 \text { (syst.) } .
$$




\section{CHAPTER 5. MEASUREMENT OF THE WEAK MIXING ANGLE}

This measurement of the effective weak-mixing angle in the predominantly $u \bar{u}$ and $d \bar{d} \rightarrow \gamma^{*} / Z \rightarrow \mu^{-} \mu^{+}$processes in proton-proton collisions is consistent with measurements in other processes $[51,52,77-80]$ as expected within the standard model.

This measurement is based on the likelihood method described by the correlated rapidity, dilepton invariant mass, and decay angle distributions in the Drell-Yan process. The evolution of this method may allow several parameters of the EWK couplings to be measured simultaneously, such as measurement of the vector and axial-vector couplings of the light quarks separately from the lepton couplings. The only change in the analysis design would require relaxing standard model constraints on the relationship between couplings in Table 5.1. Proper accounting for correlations of the systematic uncertainties in such a measurement becomes important.

With increased statistics of the Drell-Yan process at LHC, a further reduction of systematic uncertainties will become critical. Uncertainties from PDFs will reduce as better constraints on the proton model become available from LHC and elsewhere. In fact the Drell-Yan process is itself a useful input to the PDF model constraints and the methods discussed in this paper may be used to constrain the eigenvectors in the PDF model. However, one must be careful not to mix information used for PDF constraints from the Drell-Yan process to perform other measurements with the same events, unless they are introduced into an analysis in a correlated manner.

Uncertainties from the FSR model may be improved as higher-order EWK calculations are integrated with the higher-order QCD calculations of the matrix element in 


\section{CHAPTER 5. MEASUREMENT OF THE WEAK MIXING ANGLE}

the Drell-Yan process, such as, for example, the integration of POWHEG and Horace. While certain assumptions and corrections could allow us to reduce this particular error in the present analysis, we choose to be conservative since the total error is not necessarily sensitive to any one particular component and this approach reflects the present state of the tools used.

Understanding the alignment of the silicon vertex detectors on the LHC is critical for precision measurements using tracking information and it will certainly improve as LHC collaborations gain further experience. However, the present state of the CMS Tracker alignment is already excellent for this early stage of the experiment and for the measurement presented in this paper.

Finally, effects from LO assumptions in the design of the model may be further suppressed as NLO matrix elements are employed in the likelihood approach and more variables are integrated into the analysis. However, we view the current LO development as a conceptual step to develop multivariate matrix-element approaches to resonance polarization analyses which can be equally applied either to the precision measurements with the standard model processes or to potential new resonances which may appear on LHC. 

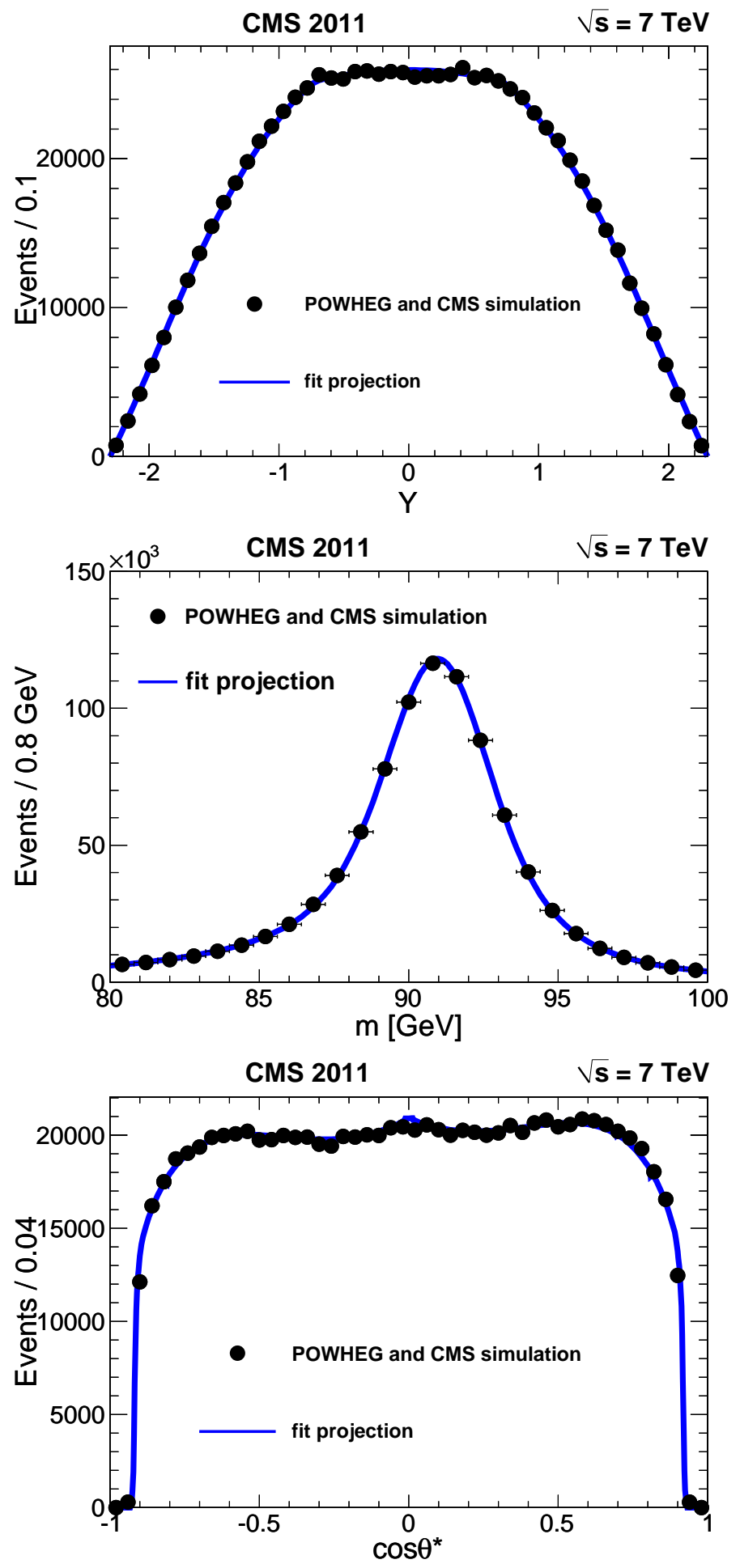

Figure 5.22: Distribution of $Y, \cos \theta^{*}, m$ in the analysis of $q \bar{q} \rightarrow \gamma^{*} / Z \rightarrow \mu^{-} \mu^{+}$events from POWHEG-based CMS detector simulation. 

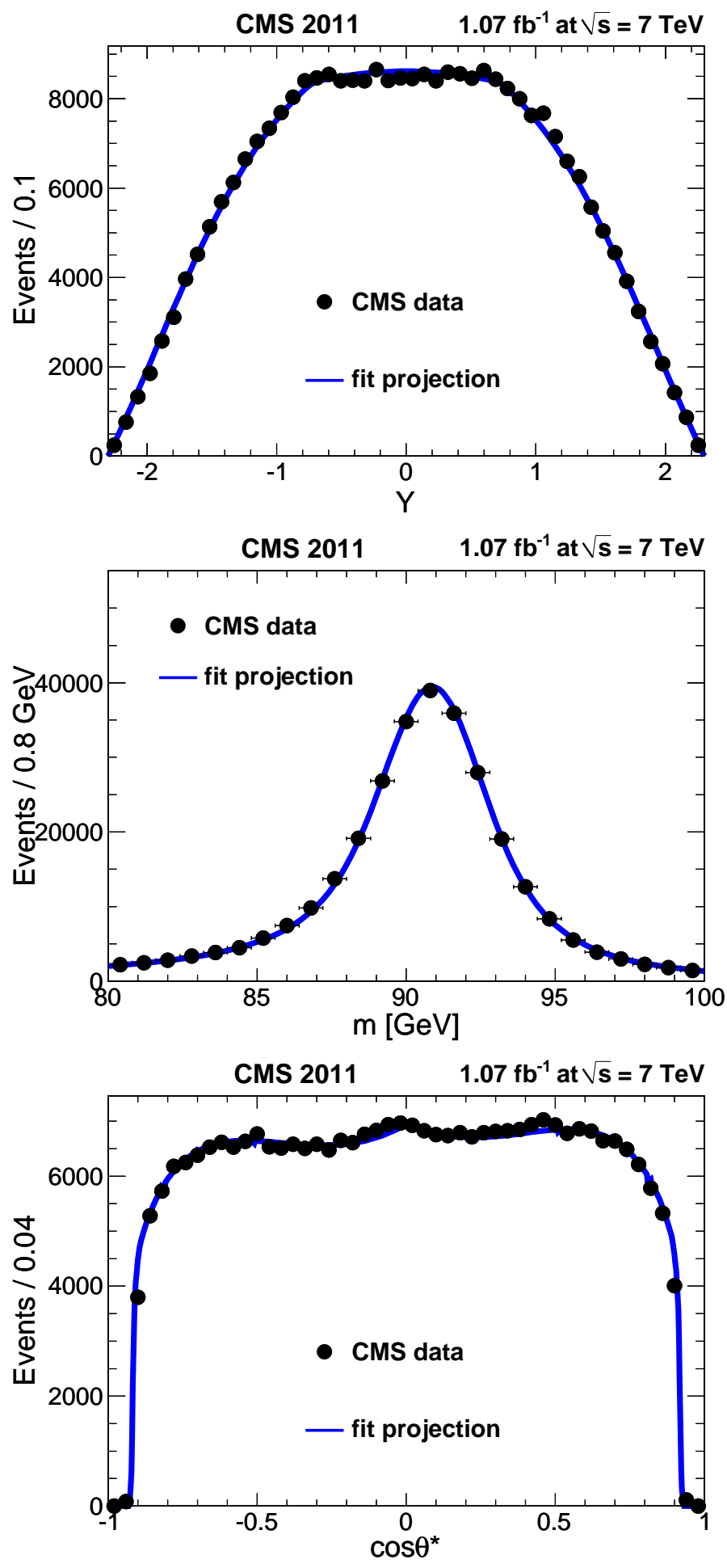

Figure 5.23: Distribution of $Y, \cos \theta^{*}, m$ in the analysis of $q \bar{q} \rightarrow \gamma^{*} / Z \rightarrow \mu^{-} \mu^{+}$events from LHC data. 


\section{Chapter 6}

\section{Study of resonances in $X \rightarrow Z Z \rightarrow 4 \ell$}

"What a bunch of cry babies...no guts, no glory, man."

- Rajesh Koothrappali, The Big Bang Theory

(in response to the claim that the LHC will create a black hole and swallow the earth)

In this chapter, the process $p p \rightarrow X \rightarrow Z Z \rightarrow 4 l$ is studied. In Section 6.1, we apply the concepts from Chapter 4 to the analysis of determining the nature of a particle $X$ using angular distributions. In Section 6.2, we examine the most recent searches for the Higgs boson at CMS. Limits are set for the search for the Higgs boson in the $p p \rightarrow H \rightarrow Z Z \rightarrow 4 l$ channel using $1.66 \mathrm{fb}^{-1}$ of CMS data, and those results are combined with the searches from many other decay channels to set the ultimate limits on the Higgs boson mass. 
CHAPTER 6. STUDIES OF RESONANCES IN $X \rightarrow Z Z \rightarrow 4 \ell$

\subsection{Spin and $C P$ determination of resonances

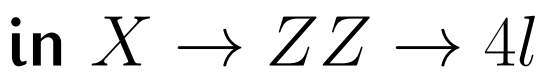

The goal of this section is to determine how well we can distinguish between various signal scenarios and how well we can determine the couplings of a new resonance to SM fields. The formalism developed in Chapter 4 is based on a generic resonance $X$ with spin $J$. In order to understand the sensitivity of the angular distributions, we perform a case study. Seven signal scenarios are chosen and we perform two types of statistical tests: hypothesis testing and parameter fitting. In the hypothesis testing, we compare our signal scenarios and use the angular distributions to quantify how well we can distinguish them. For the parameter fitting, an approach very similar to the measurement of $\sin ^{2} \theta_{W}$ is taken where we fit for the helicity fractions and phases.

\section{Case Studies}

\section{Signal and Background}

To illustrate the data analysis techniques and understand the statistical power of the proposed approaches, we choose seven signal scenarios which cover a range of spin and parity combinations. They are described in Table 6.1. The $0^{+}$scenario represents a SM Higgs-like scalar particle. The $0^{-}$scenario represents a pseudoscalar particle. The $1^{+}$scenarios and $1^{-}$represent an exotic axial-vector or vector particle, 
CHAPTER 6. STUDIES OF RESONANCES IN $X \rightarrow Z Z \rightarrow 4 \ell$

Table 6.1: List of signal scenarios used in the case study

\begin{tabular}{ccc}
\hline \hline scenario $\left(J^{P}\right)$ & $X \rightarrow Z Z$ decay parameters & $X$ production parameters \\
\hline $0^{+}$ & $a_{1} \neq 0$ in Eq. (4.1) & $g g \rightarrow X$ \\
$0^{-}$ & $a_{3} \neq 0$ in Eq. (4.1) & $g g \rightarrow X$ \\
$1^{+}$ & $g_{12} \neq 0$ in Eq. (4.3) & $q \bar{q} \rightarrow X: \rho_{11}, \rho_{12} \neq 0$ in Eq. (4.8) \\
$1^{-}$ & $g_{11} \neq 0$ in Eq. (4.3) & $q \bar{q} \rightarrow X: \rho_{11}, \rho_{12} \neq 0$ in Eq. (4.8) \\
$2_{m}^{+}$ & $g_{1}^{(2)}=g_{5}^{(2)} \neq 0$ in Eq. (4.4) & $g g \rightarrow X: g_{1}^{(2)} \neq 0$ in Eq. (4.4) \\
& $q \bar{q} \rightarrow X: \rho_{21} \neq 0$ in Eq. (4.9) \\
$2_{L}^{+}$ & $c_{2} \neq 0$ in Eq. (4.5) & $g g \rightarrow X: g_{2}^{(2)}=g_{3}^{(2)} \neq 0$ in Eq. (4.4) \\
& $q \bar{q} \rightarrow X: \rho_{21}, \rho_{22} \neq 0$ in Eq. (4.9) \\
$2^{-}$ & $g_{8}^{(2)}=g_{9}^{(2)} \neq 0$ in Eq. (4.4) & $g g \rightarrow X: g_{1}^{(2)} \neq 0$ in Eq. (4.4) \\
& & $q \bar{q} \rightarrow X: \rho_{21}, \rho_{22} \neq 0$ in Eq. (4.9) \\
\hline \hline
\end{tabular}

respectively. The $2^{+}$scenarios represent a spin-two tensor particle. In the case of $2_{m}^{+}$, the minimal couplings defined in Table 6.1 are considered and is often the most common Graviton-like resonance in the literature. In the case of $2_{L}^{+}$, we choose a longitudinally polarized Graviton-like tensor with a spin projection contribution of $J_{Z}=0$. Finally we have the $2^{-}$scenario which represents a pseudo-tensor like particle.

To perform the case studies, a Monte Carlo program ${ }^{1}$ [33] developed for this analysis was written to simulate the production and decay of spin-zero, spin-one, and spin-two resonances in hadron-hadron collisions, including all spin correlations, in the processes $g g(q \bar{q}) \rightarrow X \rightarrow Z Z \rightarrow 4 l$. A special feature of our program, that distinguishes it from other recent implementations [81], is that it includes the most general couplings of the $X$ particle to gluons and fermions in production and to $Z$ bosons in decay. The convention for the couplings used in the MC program are defined in Section 4.2. In addition, the program also includes an extension for hadronic $Z$ decays

\footnotetext{
${ }^{1}$ www.pha.jhu.edu/ spin
} 
CHAPTER 6. STUDIES OF RESONANCES IN $X \rightarrow Z Z \rightarrow 4 \ell$

to be used in the $2 l 2 q$ final state.

The spin-zero resonance is produced in collisions of gluons, and the spin-one resonance is produced in $q \bar{q}$ collisions. For the spin-two resonance, both partonic channels are included in the simulation. Since, in the general case, the relative strength of $g g X$ and $q \bar{q} X$ couplings is not known, the program allows the user to set the relative fraction of the $g g X$ and $q \bar{q} X$ couplings in production. The program also allows the user to define on-shell or off-shell production of the $X$ and $Z$ mass. The narrow width approximation is a reasonable assumption for the $Z$ bosons except for the case of a resonance $X$ below the $Z Z$ mass threshold, but it is not possible to say a priori if this also is a good approximation for the resonance $X$.

The program can output both weighted and unweighted events, depending on the requested mode of operation. Weighted events are typically used for fast calculations of simple one-dimensional distributions and for debugging purposes. Unweighted events, on the other hand, are used to interface the results of our program to programs that simulate parton showering and realistic detector response. The unweighted events are generated in the standard LHE [82] format for compatibility.

We now turn to the discussion of how backgrounds of the resonance $X$ for fourlepton production in hadron collisions are simulated. Such backgrounds arise from $Z\left(\gamma^{*}\right) Z\left(\gamma^{*}\right), Z b \bar{b}, t \bar{t}$ and $Z+$ jets where we assume that $b$-quarks decay semileptonically. Reducible backgrounds that involve $b$-decays into leptons can be controlled by requiring that leptons are isolated. Other reducible backgrounds can be strongly sup- 
CHAPTER 6. STUDIES OF RESONANCES IN $X \rightarrow Z Z \rightarrow 4 \ell$

pressed by requiring that the invariant masses of lepton pairs are close to the $Z$-boson mass, that there is no missing energy in the event, and that the four tracks originate from the same vertex near the interaction region. More discussion of techniques to suppress reducible background contributions are outlined in Section 6.2. As a result, $p p \rightarrow Z Z \rightarrow 4 l$ is the major irreducible background that survives all the possible selection requirements [83]. In this study, we restrict ourselves to $p p \rightarrow Z Z \rightarrow 4 l$ as the only background source and simulate it using Madgraph [66].

The distributions of all five angles $-\cos \theta^{*}, \Phi_{1}, \cos \theta_{1}, \cos \theta_{2}$, and $\Phi-$ for the seven models in Table 6.1 and the $p p \rightarrow Z Z \rightarrow 4 l$ background process are shown in Fig. 6.1. We note that in these studies, the azimuthal production angle we choose to use for computational convenience is $\Phi_{1}$ whereas in the algebraic expressions in Section 4.4 use the angle $\Psi$ for notational convenience. They are simply related by $\Psi=\Phi_{1}+\Phi / 2$ where all three angles are defined in Section 4.4.

These distributions were generated with our program assuming that the resonance mass is $m_{X}=250 \mathrm{GeV}$. In this section we consider $\sqrt{s}=14 \mathrm{TeV}$ proton-proton collisions and use the CTEQ6L1 parton distribution functions [54]. Note that the distributions of $\cos \theta_{1}$ and $\cos \theta_{2}$ are identical and are combined in one plot in each case. The projections of the ideal angular distributions derived in Section 4.4 agree well with simulated distributions. It is important to note that the plots in Fig. 6.1 are one-dimensional projections of a correlated five-dimensional angular distribution. From Fig. 6.1 we can see that different hypotheses can be most efficiently separated 
CHAPTER 6. STUDIES OF RESONANCES IN $X \rightarrow Z Z \rightarrow 4 \ell$
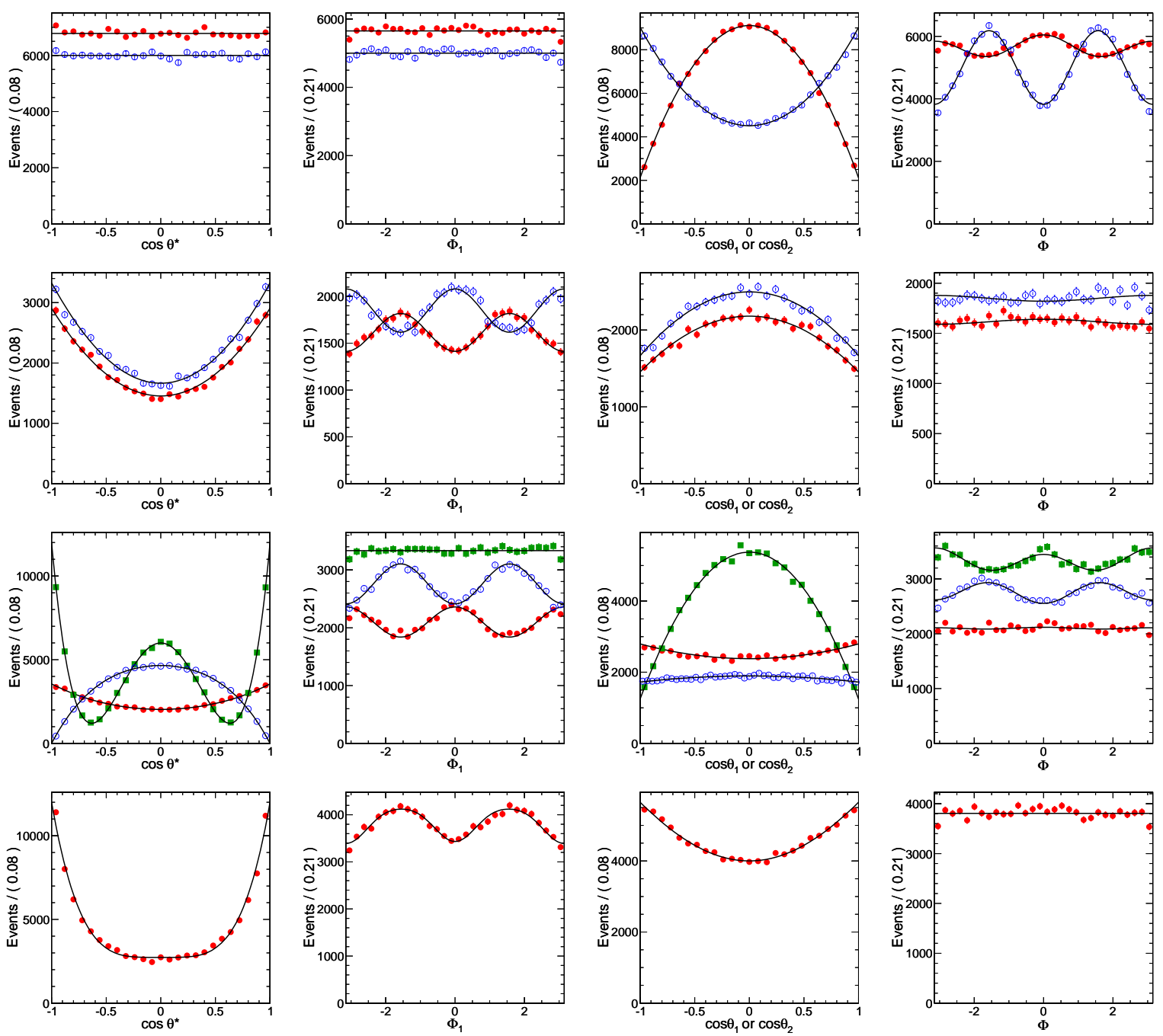

Figure 6.1: Distribution of the angular observables for the various signal scenarios in the case study and the background

if all five angles are analyzed simultaneously. Correlations in the multi-dimensional space of all angles are important for full separation power though those correlations cannot be easily illustrated. 
CHAPTER 6. STUDIES OF RESONANCES IN $X \rightarrow Z Z \rightarrow 4 \ell$

\section{Detector Effects}

For practical purposes, we perform a complete set of case studies using a standalone detector "simulation" which is not attached to any LHC experiment. Later, in addition, we produce a subset of these studies including full CMS detector simulation.

Note that, with our choice of the final state, we require measurements of the fourmomenta of all charged leptons for complete reconstruction of the event kinematics, including boosts to the rest frames of $X$ and $Z \mathrm{~s}$, where the production and helicity angles are defined. Similar to our studies of the Drell-Yan process, the main experimental effects to address are the smearing of track parameters due to finite detector resolution and geometrical detector acceptance which can sculpt the kinematic phase space of a particular process. We model the detector response in the following way. First, we assume that, for typical track momenta of order $50-250 \mathrm{GeV}$ considered in our examples, the track parameter resolution can be identified with the typical CMS tracker system resolution. Throughout this paper, we model detector resolution using the recently reported CMS track resolution parameters [24], obtained from analysis of cosmic ray data. For the three track parameters $\left(p_{T}, \phi, \theta\right)$, we apply conservative Gaussian random smearing with an rms $\Delta p_{T}=0.025 \times p_{T}+0.0001 \times p_{T}^{2}(\mathrm{GeV})$, $\Delta \phi=\Delta \theta=0.001$ (rad), and neglect resolution effects on the track origin. Within this simplified model of detector response, we observe that production and helicity angles can be measured with a typical resolution of the order of $\sim 0.01$ rad. This resolution is rather good and does not infringe on our ability to perform angular analysis. 
CHAPTER 6. STUDIES OF RESONANCES IN $X \rightarrow Z Z \rightarrow 4 \ell$

The second detector effect is the non-uniform reconstruction efficiency. We model it in a simple way assuming that only tracks produced centrally can be measured. We also assume that the detection efficiency does not change within the detector acceptance. Hence, the acceptance function is given by the step-function

$$
\mathcal{G}\left(\theta^{*}, \Phi_{1}, \theta_{1}, \theta_{2}, \Phi\right)=\prod_{i=1,2 ; q= \pm 1} \theta\left(\left|\eta_{\max }\right|-\left|\eta_{i, q}\left(\theta^{*}, \Phi_{1}, \theta_{1}, \theta_{2}, \Phi\right)\right|\right),
$$

where $\eta_{i, \pm}=\ln \cot \left(\theta_{i \pm} / 2\right)$ is the pseudorapidity of a lepton with the charge $q=$ \pm that originates from the decay of the $i^{\prime}$ th $Z$ boson, $\left|\eta_{\max }\right|=2.5$ is the maximal pseudorapidity that the tracker can reconstruct, and the product runs over the four leptons in the $X$ decay. Given the simple form of the acceptance function, it is straightforward to understand the effect of detector acceptance on angles measured in the laboratory frame, but their effect on the production and helicity angles is less obvious. To illustrate the effect of the lepton acceptance on the production angles, we plot the distributions of $\theta^{*}$ and $\Phi_{1}$ production angles for the spin-zero particle $X$ in Fig. 6.2. The mass of the resonance is $m_{X}=250 \mathrm{GeV}$ (solid points) and 1 $\mathrm{TeV}$ (open points). The lines show empirical parameterization. If these distributions are measured without any detector acceptance effects, the results are flat. Hence, the non-trivial shapes of these distributions shown in Fig. 6.2 are entirely due to an acceptance effect.

It is evident from Fig. 6.2 that the acceptance effects are very important in the analysis of data. We easily account for the acceptance effects in our MC simulation program on an event-by-event basis by using the acceptance function in Eq. 6.1, where 
CHAPTER 6. STUDIES OF RESONANCES IN $X \rightarrow Z Z \rightarrow 4 \ell$
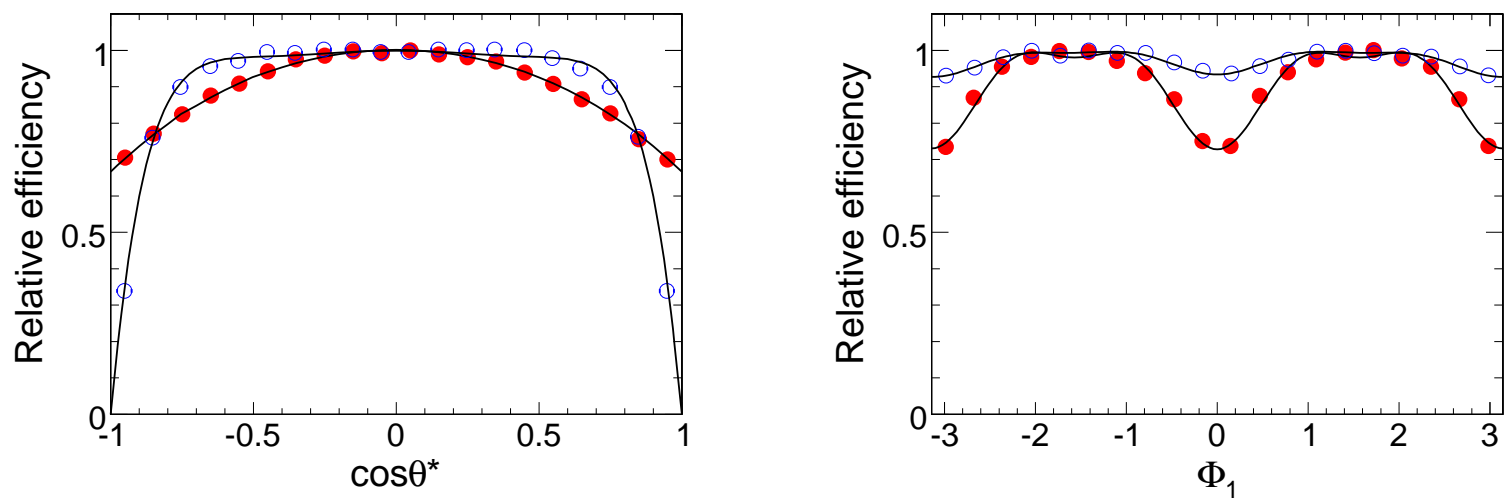

Figure 6.2: Detector acceptance effects for $\cos \theta^{*}$ and $\Phi_{1}$

we reject events if at least one lepton exceeds the maximal pseudorapidity. In order to implement the acceptance function into our likelihood function, we make certain simplifications. Based on the observation that the helicity angular distributions are independent of the production mechanism, we assume that they are not affected by the detector acceptance effects. The acceptance effect is isolated to the production angles, $\cos \theta^{*}$ and $\Phi_{1}$, and is treated as a two-dimensional function. In a more general case, acceptance effects on the helicity angles are expected to be rather mild.

\section{Data Analysis}

In this section, we now illustrate the application of the full angular analysis formalism by considering the production of a resonance $X$ and its subsequent decay to two $Z$ bosons. We use the $M C$ simulation discussed above to generate both signal and background events, and apply the multivariate fitting technique introduced in 
CHAPTER 6. STUDIES OF RESONANCES IN $X \rightarrow Z Z \rightarrow 4 \ell$

Section 5.3 to extract as much information about the produced resonances as possible. We point out that, because our analysis is general and because it is not based on any particular model of BSM physics, it is not possible to accurately predict how many $X$ particles are produced, for a given luminosity. Instead, we find it more reasonable to assume a certain number of signal and background events, reconstructed by an LHC experiment. This number of events should be sufficiently large to enable a meaningful angular analysis, yet it should be small enough, to be relevant for the situation soon after the resonance observation. The statistical power of the method can be easily extrapolated to a different number of resonance events.

As we explain below, we perform two different analyses in this paper, for which we choose to have 30 and 150 signal events, respectively. Roughly, this corresponds [?,?] to the number of SM Higgs boson events $p p \rightarrow H \rightarrow Z Z \rightarrow 4 l^{ \pm}, l=e, \mu$, detected by one LHC experiment with 5 or $25 \mathrm{fb}^{-1}$ integrated luminosity, for a Higgs boson mass $m_{H}=250 \mathrm{GeV}$, at a proton-proton center-of-mass energy of $14 \mathrm{TeV}$. Of course, the actual production rate of exotic particles may be larger or smaller; we choose 30 and 150 events merely for illustration purposes.

We consider two mass points, the low mass $m_{X}=250 \mathrm{GeV}$ and the relatively high mass $m_{X}=1 \mathrm{TeV}$. The background size is chosen according to the above LHC integrated luminosity convention, which leaves the high-mass scenario $m_{X}=1 \mathrm{TeV}$ with essentially no background, and the low-mass scenario with about 24 background events in the $m_{X}$ mass window between 230 and $270 \mathrm{GeV}$ [33]. The exact number of 
CHAPTER 6. STUDIES OF RESONANCES IN $X \rightarrow Z Z \rightarrow 4 \ell$

background events is not crucial for our analysis because we are mostly interested in understanding how well different signal hypotheses can be disentangled, rather than how well a signal can be separated from the background.

We now imagine that 30 non-SM resonance-like events have been experimentally observed and we need to understand their origin. As the very first step, we may want to find out if the observed events are described by a resonance with particular quantum numbers and a particular structure of interactions with the SM particles. To do this, we compute a confidence level at which one resonance hypothesis with a specific set of quantum numbers can be separated from another. For example, we may ask if an observed resonance looks more like a SM Higgs boson or more like a graviton with minimal couplings to SM fields. We use our MC event simulation to check how well separation of the two hypotheses can be done in practice. Once we have a good idea about the nature of the resonance $X$, we can proceed to a more statistically demanding analysis whose goal is to determine all the parameters which describe the angular distributions within a particular hypothesis.

The second approach clearly supersedes the first one and is the most optimal way to analyze data. Indeed, in the extreme version of the second approach, everything mass, width, spin, parity, and polarization parameters of the particle $X$ - is obtained through a single fitting process. The shortcoming of this approach is that it is very demanding statistically, since a large sample of events is required to enable simultaneous determination of a large number of signal parameters with decent precision. 
CHAPTER 6. STUDIES OF RESONANCES IN $X \rightarrow Z Z \rightarrow 4 \ell$

For the illustration of the second approach, we require that that 150 signal events are available. We note that our methods are general enough to implement either of the two approaches described above. The only practical difference in application of these methods is that the polarization parameters are either fixed or left as free parameters in the fit.

We use an unbinned extended maximum-likelihood (ML) fit to extract simultaneously the signal and background yields, the background shape, and the parameters of the signal angular distributions. The likelihood function for $N$ candidate events is written as

$$
\mathcal{L}=\exp \left(-n_{\mathrm{sig}, J}-n_{\mathrm{bkg}}\right) \prod_{i}^{N}\left(n_{\mathrm{sig}, J} \times \mathcal{P}_{\mathrm{sig}, J}\left(\vec{x}_{i} ; \vec{\zeta}_{J} ; \vec{\xi}\right)+n_{\mathrm{bkg}} \times \mathcal{P}_{\mathrm{bkg}}\left(\vec{x}_{i} ; \vec{\xi}\right)\right)
$$

where $n_{\mathrm{sig}, J}$ is the number of signal events for a resonance of spin $J, n_{\mathrm{bkg}}$ is the number of background events and $\mathcal{P}\left(\vec{x}_{i} ; \vec{\zeta} ; \vec{\xi}\right)$ is the probability density function for signal or background. Each event candidate $i$ is characterized by a set of six observables $\vec{x}_{i}=\left\{m_{Z Z}, \quad \cos \theta^{*}, \Phi_{1}, \quad \cos \theta_{1}, \quad \cos \theta_{2}, \Phi\right\}_{i}$. The number of observables can be extended or reduced, depending on the desired fit. The signal polarization parameters $\left\{f_{\lambda_{1} \lambda_{2}}, \phi_{\lambda_{1} \lambda_{2}}, f_{z m}\right\}$ are collectively denoted by $\vec{\zeta}_{J}$, and the remaining parameters by $\vec{\xi}$. When several decay channels must be combined, the joint likelihood in Eq. 6.2 is the natural way to achieve this. In the current analysis, a combination of all lepton channels with electrons and muons in the $Z Z$ decays is assumed.

For the signal, the probability density function $\mathcal{P}_{\text {sig, } J}\left(\vec{x}_{i} ; \vec{\zeta}_{J} ; \vec{\xi}\right)$ is taken to be a product of a function of the angles and a function of the resonance mass. For the 
CHAPTER 6. STUDIES OF RESONANCES IN $X \rightarrow Z Z \rightarrow 4 \ell$

background, the probability density function is a product of uncorrelated functions for each observable. As long as the results of the generated experiments are unbiased, small correlations are acceptable. It is an approximation of our approach that the background angular distributions are not correlated, which we find acceptable for the current analysis.

The angular probability density function for the signal is the ideal distribution in Eq. 4.30, multiplied by an empirically determined acceptance function $\mathcal{G}\left(\cos \theta^{*}, \Phi_{1}, \cos \theta_{1}, \cos \theta_{2}, \Phi\right)$, We approximate the acceptance function by $\mathcal{G}=$ $\mathcal{G}^{(1)}\left(\cos \theta^{*}\right) \times \mathcal{G}^{(2)}\left(\Phi_{1}\right)$, neglecting all correlations between different angles. The parameterization of the signal acceptance functions $\mathcal{G}^{(1,2)}$ is compared with simulated distributions in Fig. 6.2. The acceptance function $\mathcal{G}$ is included in the overall probability density function.

It is important to point out that one can do a better job of modeling if all that is needed is to understand how well a particular hypothesis describes experimental data. Since we test a specific hypothesis, ratios of helicity amplitudes are fixed. Hence, it is possible to determine the likelihood function numerically through the $M C$ simulation of the full five-dimensional angular distribution, including the detector effects, instead of using an analytical parameterization. Effectively, this requires storing a normalized five-dimensional histogram with fine binning. A similar approach can be used for background parameterization. However, it is more difficult to employ MC computation of the signal probability density, if we want to treat all the parameters, including 
CHAPTER 6. STUDIES OF RESONANCES IN $X \rightarrow Z Z \rightarrow 4 \ell$

helicity fractions, as free parameters in the fit. A straightforward use of the $M C$ program would require generating and storing probability distributions for a large set of independent polarization parameters. Clearly, for a large enough number of parameters to be fitted, this procedure becomes impractical. For the purposes of our studies, we find the simplified analytical approach to be acceptable since, as we have verified with our MC program, biases in the results are small.

The only other kinematic observable that is used in the fit in addition to the angles is the $Z Z$ invariant mass $m_{Z Z}$. The background $m_{Z Z}$ distribution is parameterized with a polynomial and its shape can be left unconstrained in the fit. We make the following assumptions about the mass and the width of the $X$ resonance. We assume that its width is negligible compared to the detector resolution effects, which are estimated to be about $3.5 \mathrm{GeV}$ at $m_{X}=250 \mathrm{GeV}$ and $23 \mathrm{GeV}$ at $m_{X}=1 \mathrm{TeV}$ with our simulation. The exact value of the resolution is only relevant for signal and background separation at the lower mass; the values quoted above are typical for both muon and electron final states. Hence, we describe the signal mass distribution with a Gaussian function of a known width. Should the resonance have a finite width that is larger than detector resolution, we can incorporate it into our analysis by replacing the Gaussian function with a convolution of the Breit-Wigner and Gaussian distributions. However, since the mass distribution has little, if any, influence on the resonance spin and its couplings to SM fields, a detailed study of mass resolution issues is left for later discussion.

The mass of the resonance $m_{X}$ is assumed to be known and is fixed in the fit. This 
CHAPTER 6. STUDIES OF RESONANCES IN $X \rightarrow Z Z \rightarrow 4 \ell$
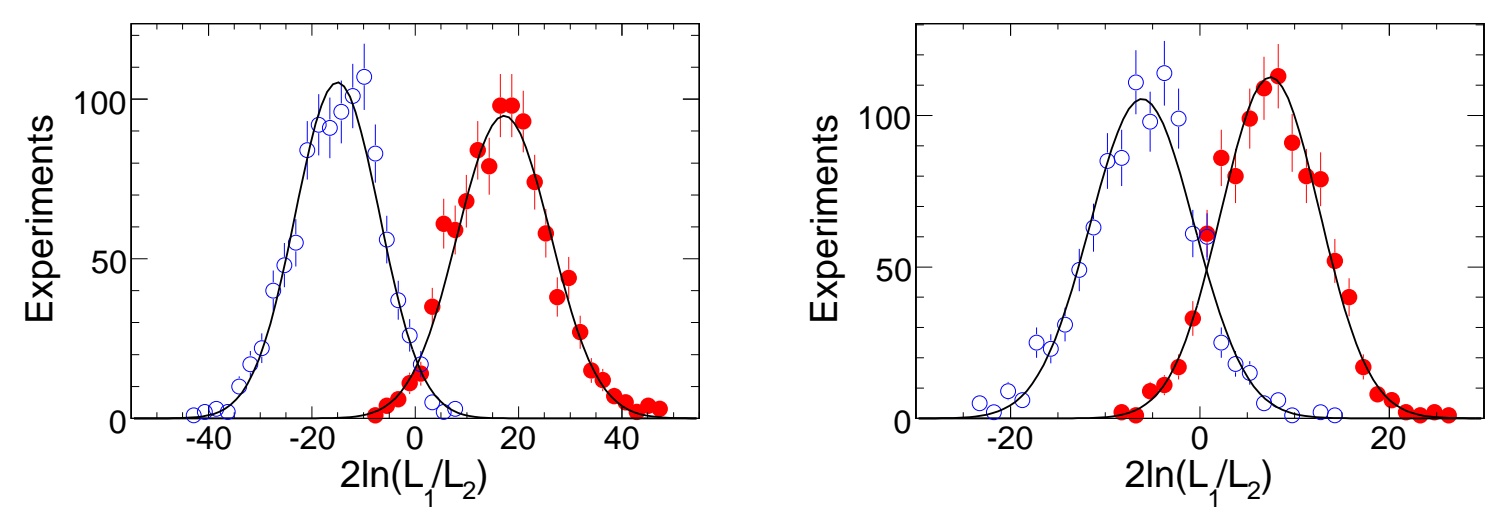

Figure 6.3: Separations of signal hypothesis for $0^{+}$and $0^{-}$(left) and $0^{+}$and $2_{m}^{+}$(right) assumption only affects the signal and background separation and requires certain care in the interpretation of the discovery significance. Detailed study of the separation of signal background is left for further examination, but we consider a simple example. Consider 30 events due to the SM Higgs-like resonance at $250 \mathrm{GeV}$. If only $m_{z z}$ is employed in the fit, the measured signal significance is $5.7 \sigma$ and it increases by nearly twenty percent if also angular variables are included in the fit. Here significance is calculated from $2 \ln \left(\mathcal{L}_{1} / \mathcal{L}_{2}\right)$ using the likelihood ratio for two scenarios with signal and with pure background, analogous to the technique discussed below, and corresponds to the Gaussian probability for pure background to fluctuate to the observed signal yield.

We are now in position to discuss the results of our analysis. As an illustration of the first approach, in Fig. 6.3, we plot the quantity $2 \ln \left(\mathcal{L}_{1} / \mathcal{L}_{2}\right)$ for two sets of 1000 MC experiments generated with the models $0^{+}$and $0^{-}$(left plot) or $0^{+}$and $2_{m}^{+}$(right plot), as listed in Table 6.1. Each experiment has on average 24 background and 30 
CHAPTER 6 . STUDIES OF RESONANCES IN $X \rightarrow Z Z \rightarrow 4 \ell$

Table 6.2: Results of the hypothesis separation of the seven scenarios listed in Table 6.1 for $m_{X}=250 \mathrm{GeV}$.

\begin{tabular}{ccccccc}
\hline \hline & $0^{-}$ & $1^{+}$ & $1^{-}$ & $2_{m}^{+}$ & $2_{L}^{+}$ & $2^{-}$ \\
\hline $0^{+}$ & $0.0 / 0.0 / 3.9 / 4.1 / 4.1$ & $0.8 / 1.0 / 1.8 / 1.9 / 2.3$ & $0.9 / 1.0 / 2.5 / 2.6 / 2.6$ & $0.8 / 0.9 / 2.4 / 2.5 / 2.8$ & $2.6 / 2.6 / 0.0 / 2.6 / 2.6$ & $1.6 / 1.7 / 2.4 / 3.0 / 3.3$ \\
$0^{-}$ & & $0.8 / 1.2 / 2.8 / 3.0 / 3.1$ & $0.9 / 1.0 / 2.5 / 2.8 / 3.0$ & $0.8 / 0.8 / 1.7 / 2.0 / 2.4$ & $2.9 / 2.9 / 4.1 / 4.8 / 4.8$ & $1.6 / 1.7 / 2.0 / 2.7 / 2.9$ \\
$1^{+}$ & & $0.0 / 1.1 / 1.1 / 1.2 / 2.2$ & $0.1 / 1.2 / 1.3 / 1.4 / 2.6$ & $2.8 / 2.8 / 1.9 / 3.5 / 3.6$ & $2.5 / 2.4 / 1.2 / 2.7 / 2.9$ \\
$1^{-}$ & & & $0.1 / 0.1 / 1.3 / 1.5 / 1.8$ & $2.8 / 2.9 / 2.5 / 3.8 / 3.8$ & $2.5 / 2.6 / 0.6 / 2.8 / 3.4$ \\
$2_{m}^{+}$ & & & & $2.9 / 2.9 / 2.6 / 3.6 / 3.8$ & $2.3 / 2.5 / 0.5 / 2.5 / 3.2$ \\
$2_{L}^{+}$ & & & & & $3.6 / 3.6 / 2.5 / 4.2 / 4.3$ \\
\hline \hline
\end{tabular}

Table 6.3: Results of the hypothesis separation of the seven scenarios listed in Table 6.1 for $m_{X}=1 \mathrm{TeV}$.

\begin{tabular}{|c|c|c|c|c|c|c|}
\hline & $0^{-}$ & $1^{+}$ & $1^{-}$ & $2_{m}^{+}$ & $2_{L}^{+}$ & $2^{-}$ \\
\hline $0^{+}$ & $0.0 / 0.0 / 3.9 / 4.0 / 4.1$ & $0.4 / 0.6 / 2.2 / 2.2 / 2.4$ & $0.3 / 0.5 / 2.2 / 2.1 / 2.4$ & $1.2 / 1.1 / 2.8 / 2.7 / 2.8$ & $1.0 / 1.0 / 0.0 / 1.2 / 1.2$ & $1.2 / 1.0 / 2.0 / 2.7 / 2.8$ \\
\hline $0^{-}$ & & $0.5 / 0.9 / 2.8 / 2.9 / 3.1$ & $0.5 / 0.8 / 2.7 / 2.9 / 3.1$ & $1.4 / 1.5 / 1.4 / 2.1 / 2.1$ & $1.1 / 1.1 / 4.0 / 4.3 / 4.5$ & $0.8 / 0.9 / 2.9 / 2.8 / 2.9$ \\
\hline $1^{+}$ & & & $0.0 / 1.1 / 1.2 / 1.2 / 2.3$ & $0.9 / 0.9 / 2.0 / 2.0 / 2.3$ & $1.3 / 1.4 / 2.1 / 2.4 / 2.6$ & $1.3 / 1.3 / 1.1 / 1.8 / 2.0$ \\
\hline $1^{-}$ & & & & $0.9 / 0.9 / 2.0 / 2.0 / 2.1$ & $1.3 / 1.3 / 2.2 / 2.5 / 2.7$ & 1.4/1.6/0.0/1.4/2.2 \\
\hline $2_{m}^{+}$ & & & & & $1.7 / 1.7 / 3.0 / 3.2 / 3.2$ & $2.1 / 2.0 / 2.1 / 2.6 / 2.6$ \\
\hline $2_{L}^{+}$ & & & & & & $1.5 / 1.5 / 2.1 / 3.3 / 3.3$ \\
\hline
\end{tabular}

signal events, Poisson distributed. These events are generated at $m_{X}=250 \mathrm{GeV}$ in 1000 statistically independent experiments. The likelihood $\mathcal{L}_{k}$ in Eq. 6.2 is evaluated independently for each hypothesis $k$. The discussion of the statistical interpretation of $2 \ln \left(\mathcal{L}_{1} / \mathcal{L}_{2}\right)$ was given recently in Ref. [84]. The distribution of $2 \ln \left(\mathcal{L}_{1} / \mathcal{L}_{2}\right)$ is expected to peak at positive values for events generated according to model one (on average $\mathcal{L}_{1}>\mathcal{L}_{2}$ ) and at negative values for events generated according to model two (on average $\mathcal{L}_{2}>\mathcal{L}_{1}$ ). From Fig. 6.3, we extract the quantity $\mathcal{S}$ in the following way. We find the point beyond which the right-side tail of the left histogram and the left-side tail of the right histogram have equal areas. These areas correspond to the one-sided Gaussian probability outside of the $\mathcal{S} / 2 \sigma$ range. If the two histograms in Fig. 6.3 were perfectly Gaussian distributed with unit width, then $\mathcal{S}$ corresponds to the separation between the peaks of the two distributions. The effective signal hypothesis separation 
CHAPTER 6. STUDIES OF RESONANCES IN $X \rightarrow Z Z \rightarrow 4 \ell$

for $0^{+}$and $0^{-}$is $4.1 \sigma$ and for $0^{+}$and $2_{m}^{+}$is $2.8 \sigma$.

The above procedure is repeated for all combinations of seven hypotheses listed in Table 6.1, for $m_{X}=250 \mathrm{GeV}$ and $m_{X}=1 \mathrm{TeV}$, and results are presented in Tables 6.2 and 6.3. In those tables, we show the increase in the separation power between two hypotheses if more information about angular distributions is included in the likelihood fit. It is natural to refer to a likelihood fit that includes $n$ angular variables as $n$-dimensional or $n \mathrm{D}$; the results of the $1 \mathrm{D}, 2 \mathrm{D}, 3 \mathrm{D}, 4 \mathrm{D}$, and $5 \mathrm{D}$ fits (1D/2D/3D/4D/5D) are shown in Tables 6.2 and 6.3. In the $1 \mathrm{D}$ fit, only the $\cos \theta^{*}$ angular distribution is used; in the 2D fit, the $\left(\cos \theta^{*}, \Phi_{1}\right)$ distribution is included; in the 3D fit, we have $\left(\cos \theta_{1}, \cos \theta_{2}, \Phi\right)$; in the $4 \mathrm{D}$ fit, the $\left(\cos \theta^{*}, \cos \theta_{1}, \cos \theta_{2}, \Phi\right)$ distribution is used; and finally in 5D fit, the $\left(\cos \theta^{*}, \Phi_{1}, \cos \theta_{1}, \cos \theta_{2}, \Phi\right)$ distribution is employed. We note that because we generate a finite number of experiments (1000), the statistical uncertainties on the values of $\mathcal{S}$ reported in Tables 6.2 and 6.3 are typically in the range $0.1-0.2$. This explains some of the small discrepancies in the values quoted. For example, one could expect the separation of $0^{+}$and $0^{-}$hypotheses to be the same in 3D, 4D, and 5D cases. However, statistical fluctuations and better background suppression make the 4D and 5D cases look better.

It is clear from Tables 6.2 and 6.3 that the multidimensional angular analysis allows efficient separation of different spin hypotheses and, therefore, is a very powerful approach to spin determination. While separations $\mathcal{S}=2$ are typically achieved, there are important cases when larger separations are possible. In particular, we 
CHAPTER 6. STUDIES OF RESONANCES IN $X \rightarrow Z Z \rightarrow 4 \ell$

observe $\mathcal{S}=4$ for the separation of $0^{+}$and $0^{-}$hypotheses, which might be useful even for the SM Higgs boson if its mass exceeds $2 m_{z}$. Also, the separation of the spin-two with the minimal couplings hypothesis from the spin-two with longitudinal amplitude dominance hypothesis is close to $\mathcal{S}=3$, for any production mechanism scenario. In all cases, the results of the 5D fit provide the best separation power and supersede the other fits.

A glance at Tables 6.2 and 6.3 is sufficient to recognize that there are cases when different hypotheses about the nature of the resonance cannot be separated $(\mathcal{S}=0)$. For example, the production angles $\cos \theta^{*}$ and $\Phi_{1}$ provide no separation between the two spin-zero scenarios $\left(0^{+}\right.$and $\left.0^{-}\right)$due to the absence of spin correlations. At the same time, the three helicity angle $\left(\cos \theta_{1}, \cos \theta_{2}, \Phi\right)$ distributions are identical for the $0^{+}$and $2_{L}^{+}$hypotheses, therefore the $3 \mathrm{D}$ case results in $\mathcal{S}=0$ as well. The only source of separation in this case is the production angle. We have not considered any example with no separation from either production or helicity angles, but such situation is possible when spin-zero and spin-two scenarios with $f_{z 1}=f_{z 2}=0.4$ are considered, as we discussed earlier. A hint at this situation is observed in the decreased $\mathcal{S}$-value that characterizes the separation of $0^{+}$and $2_{L}^{+}$hypotheses when the mass of the resonance increases from $250 \mathrm{GeV}$ to $1 \mathrm{TeV}$. This is the consequence of the fact that at $1 \mathrm{TeV}$ we allow $q \bar{q}$ production. This leads to $f_{z 1}=0.25$ and $f_{z 2}=0.30$, which is closer to the unpolarized case. As the result, the $\mathcal{S}$-value decreases dramatically.

The most general analysis of an observed resonance requires that the likelihood in 
CHAPTER 6. STUDIES OF RESONANCES IN $X \rightarrow Z Z \rightarrow 4 \ell$

Eq. 6.2 is maximized with respect to the signal yield and all signal angular parameters, $\vec{\zeta}_{J}$, as well as with respect to unconstrained background parameters $\vec{\xi}$. This requires a large number of free parameters even for signal alone. The fit delivers the most probable values of these parameters and the covariance matrix which describes their uncertainties and correlations ${ }^{2}$. In principle, it is possible to pursue this strategy within our approach, but for the fit to converge with high success rate, one may need large enough event sample. Therefore, for the illustration of the fit technique, we consider a limited procedure where only one spin hypothesis is considered at a time, but all other parameters relevant for that particular spin hypothesis are allowed to float in the fit.

In Tables 6.4 and 6.5, this technique is illustrated with the spin-zero hypothesis with samples generated according to the $0^{+}$and $0^{-}$scenarios, respectively. We show the results of the fit with both "perfect" and realistic detectors. In Fig. 6.4 we show distributions for two parameters in this analysis for a set of 1000 generated experiments. On the top, a distribution of the number of fitted signal events $n_{\text {sig }}$ (left) and the fraction of transverse component in the decay amplitude $\left(f_{++}+f_{--}\right)$(right) is shown for the $0^{+}$signal hypothesis corresponding to Table 6.4. The bottom of the plot shows the top parameters normalized by the fit errors. The means $\mu$ and widths $w$ of these distributions are shown in Table 6.4 as $\mu \pm w$. These values are the most probable central values and errors of a measurement, but they are subject to statistical fluctu-

\footnotetext{
${ }^{2}$ Knowledge of the full dependence of $-2 \ln \mathcal{L}$ on all the parameters is even more beneficial.
} 
CHAPTER 6. STUDIES OF RESONANCES IN $X \rightarrow Z Z \rightarrow 4 \ell$

ation in each given experiment. Normalized distributions in Fig. 6.4 illustrate proper error estimates in the fit. In Tables 6.6 and 6.7, the result of a similar study with the spin-one hypotheses $1^{+}$and $1^{-}$are presented. Finally, in Tables 6.7, 6.8, and 6.9 we illustrate the fit with the spin-two hypotheses for $2_{m}^{+}, 2_{L}^{+}$, and $2^{-}$, respectively, where we also restrict the hypothesis to the definite parity to reduce the number of free parameters. We used 1000 generated samples of 150 signal events each, Poisson distributed, and the corresponding number of background events, per sample. In all cases the set of observables includes $m_{z Z}, \cos \theta^{*}, \cos \theta_{1}, \cos \theta_{2}$, and $\Phi$. There are several cases where contribution of a certain helicity amplitude is either exactly zero (e.g. $A_{00}$ in the $0^{-}$case) or negligible (e.g. $A_{++}$in the $2_{m}^{+}$case). Since measuring the phase of that amplitude becomes impossible, we leave it as "free" in the tables. Table 6.4: Results of the fit for the free parameters of the spin-zero hypothesis with generated samples of SM Higgs-like $X$ corresponding to $0^{+}$.

\begin{tabular}{lcccccc}
\hline \hline & \multicolumn{3}{c}{$m_{X}=250 \mathrm{GeV}$} & \multicolumn{2}{c}{$m_{X}=1 \mathrm{TeV}$} \\
& generated & \multicolumn{2}{c}{ fitted } & generated & \multicolumn{2}{c}{ fitted } \\
& & without detector & with detector & & without detector & with detector \\
\hline$n_{\text {sig }}$ & 150 & $150 \pm 13$ & $153 \pm 15$ & 150 & $150 \pm 12$ & $152 \pm 12$ \\
$\left(f_{++}+f_{--}\right)$ & 0.208 & $0.21 \pm 0.07$ & $0.23 \pm 0.08$ & 0.000 & $0.00 \pm 0.03$ & $0.00 \pm 0.03$ \\
$\left(f_{++}-f_{--}\right)$ & 0.000 & $0.01 \pm 0.13$ & $0.01 \pm 0.14$ & 0.000 & $0.00 \pm 0.02$ & $0.00 \pm 0.02$ \\
$\left(\phi_{++}+\phi_{--}\right)$ & $2 \pi$ & $6.30 \pm 1.46$ & $6.39 \pm 1.54$ & $2 \pi$ & free & free \\
$\left(\phi_{++}-\phi_{--}\right)$ & 0 & $0.00 \pm 1.06$ & $0.01 \pm 1.09$ & 0 & free & free \\
\hline \hline
\end{tabular}

Table 6.5: Results of the fit for the free parameters of the spin-zero hypothesis with generated samples of a pseudo-scalar $X$ corresponding to $0^{-}$.

\begin{tabular}{lcccccc}
\hline \hline & \multicolumn{3}{c}{$m_{X}=250 \mathrm{GeV}$} & \multicolumn{3}{c}{$m_{X}=1 \mathrm{TeV}$} \\
& generated & \multicolumn{2}{c}{ fitted } & generated & \multicolumn{2}{c}{ fitted } \\
& & without detector & with detector & & without detector & with detector \\
\hline$n_{\text {sig }}$ & 150 & $150 \pm 13$ & $151 \pm 15$ & 150 & $151 \pm 12$ & $150 \pm 13$ \\
$\left(f_{++}+f_{--}\right)$ & 1.000 & $1.00 \pm 0.05$ & $1.00 \pm 0.06$ & 1.000 & $1.00 \pm 0.05$ & $1.00 \pm 0.06$ \\
$\left(f_{++}-f_{--}\right)$ & 0.000 & $0.00 \pm 0.35$ & $0.00 \pm 0.40$ & 0.000 & $0.00 \pm 0.31$ & $-0.01 \pm 0.32$ \\
$\left(\phi_{++}+\phi_{--}\right)$ & $\mathrm{N} / \mathrm{A}$ & free & free & $\mathrm{N} / \mathrm{A}$ & free & free \\
$\left(\phi_{++}-\phi_{--}\right)$ & $\pi$ & $3.15 \pm 0.31$ & $3.14 \pm 0.41$ & $\pi$ & $3.15 \pm 0.31$ & $3.14 \pm 0.33$ \\
\hline \hline
\end{tabular}


CHAPTER 6. STUDIES OF RESONANCES IN $X \rightarrow Z Z \rightarrow 4 \ell$

Table 6.6: Results of the fit for the free parameters of the spin-one hypothesis with generated samples of an exotic pseudo-vector $X$ corresponding to $1^{+}$.

\begin{tabular}{lcccccc}
\hline \hline & \multicolumn{3}{c}{$m_{X}=250 \mathrm{GeV}$} & \multicolumn{3}{c}{$m_{X}=1 \mathrm{TeV}$} \\
& generated & \multicolumn{2}{c}{ fitted } & generated & \multicolumn{2}{c}{ fitted } \\
& & without detector & with detector & & without detector & with detector \\
\hline$n_{\text {sig }}$ & 150 & $150 \pm 13$ & $152 \pm 15$ & 150 & $151 \pm 12$ & $152 \pm 13$ \\
$f_{+0}$ & 0.250 & $0.26 \pm 0.15$ & $0.26 \pm 0.17$ & 0.250 & $0.25 \pm 0.16$ & $0.25 \pm 0.15$ \\
$\left(\phi_{+0}-\phi_{0-}\right)$ & 0 & $0.02 \pm 0.73$ & $0.01 \pm 0.86$ & 0 & $-0.03 \pm 0.67$ & $-0.03 \pm 0.77$ \\
\hline \hline
\end{tabular}

Table 6.7: Results of the fit for the free parameters of the spin-one hypothesis with generated samples of an exotic vector $X$ corresponding to $1^{-}$.

\begin{tabular}{lcccccc}
\hline \hline & \multirow{3}{*}{$\begin{array}{c}m_{X}=250 \mathrm{GeV} \\
\text { fitted }\end{array}$} & generated & \multicolumn{2}{c}{$m_{X}=1 \mathrm{TeV}$} \\
& & \multicolumn{2}{c}{ fitted } \\
& & without detector & with detector & & without detector & with detector \\
\hline$n_{\text {sig }}$ & 150 & $150 \pm 13$ & $152 \pm 16$ & 150 & $151 \pm 12$ & $152 \pm 13$ \\
$f_{+0}$ & 0.250 & $0.24 \pm 0.15$ & $0.24 \pm 0.16$ & 0.250 & $0.24 \pm 0.17$ & $0.25 \pm 0.15$ \\
$\left(\phi_{+0}-\phi_{0-}\right)$ & $\pi$ & $3.14 \pm 0.72$ & $3.18 \pm 0.76$ & $\pi$ & $3.14 \pm 0.71$ & $3.14 \pm 0.69$ \\
\hline \hline
\end{tabular}

Table 6.8: Results of the fit for the free parameters of the spin-two hypothesis with positive parity with generated samples of an exotic $X$ corresponding to $2_{m}^{+}$.

\begin{tabular}{lcccccc}
\hline \hline & \multicolumn{3}{c}{$m_{X}=250 \mathrm{GeV}$} & \multicolumn{3}{c}{$m_{X}=1 \mathrm{TeV}$} \\
fitted & generated & \multicolumn{2}{c}{ fitted } \\
& & \multicolumn{2}{c}{ generated } & without detector & with detector \\
\hline$n_{\text {sig }}$ & 150 & $150 \pm 13$ & $151 \pm 16$ & 150 & $151 \pm 12$ & $153 \pm 13$ \\
$f_{z 2}$ & 1.000 & $1.00 \pm 0.17$ & $0.84 \pm 0.17$ & 0.750 & $0.75 \pm 0.12$ & $0.80 \pm 0.10$ \\
$f_{z 1}$ & 0.000 & $0.00 \pm 0.19$ & $0.00 \pm 0.25$ & 0.250 & $0.25 \pm 0.14$ & $0.16 \pm 0.15$ \\
$f_{++}$ & 0.013 & $0.01 \pm 0.04$ & $0.00 \pm 0.05$ & 0.000 & $0.00 \pm 0.05$ & $0.00 \pm 0.05$ \\
$f_{+-}$ & 0.282 & $0.28 \pm 0.04$ & $0.31 \pm 0.05$ & 0.445 & $0.44 \pm 0.06$ & $0.44 \pm 0.04$ \\
$f_{+0}$ & 0.075 & $0.07 \pm 0.04$ & $0.06 \pm 0.05$ & 0.000 & $0.01 \pm 0.06$ & $0.01 \pm 0.06$ \\
$\phi_{++}$ & 0 & $0.00 \pm 1.75$ & $0.04 \pm 1.76$ & 0 & free & free \\
\hline \hline
\end{tabular}

Table 6.9: Results of the fit for the free parameters of the spin-two hypothesis with positive parity with generated samples of an exotic $X$ corresponding to $2_{L}^{+}$.

\begin{tabular}{ccccccc}
\hline \hline & \multicolumn{3}{c}{$m_{X}=250 \mathrm{GeV}$} & \multicolumn{3}{c}{$m_{X}=1 \mathrm{TeV}$} \\
fitted & generated & \multicolumn{2}{c}{ fitted } \\
& & \multicolumn{2}{c}{ generated } & without detector & with detector \\
\hline$n_{\text {sig }}$ & 150 & $150 \pm 13$ & $154 \pm 15$ & 150 & $151 \pm 12$ & $152 \pm 13$ \\
$f_{z 2}$ & 0.400 & $0.40 \pm 0.07$ & $0.33 \pm 0.10$ & 0.300 & $0.30 \pm 0.08$ & $0.34 \pm 0.09$ \\
$f_{z 1}$ & 0.000 & $0.00 \pm 0.03$ & $0.00 \pm 0.04$ & 0.250 & $0.25 \pm 0.07$ & $0.23 \pm 0.07$ \\
$f_{++}$ & 0.104 & $0.10 \pm 0.04$ & $0.06 \pm 0.05$ & 0.000 & $0.00 \pm 0.01$ & $0.00 \pm 0.02$ \\
$f_{+-}$ & 0.000 & $0.00 \pm 0.04$ & $0.04 \pm 0.05$ & 0.000 & $0.00 \pm 0.01$ & $0.00 \pm 0.03$ \\
$f_{+0}$ & 0.000 & $0.00 \pm 0.02$ & $0.00 \pm 0.02$ & 0.000 & $0.00 \pm 0.02$ & $0.00 \pm 0.02$ \\
$\phi_{++}$ & $\pi$ & $3.20 \pm 0.75$ & $3.17 \pm 0.71$ & $\pi$ & free & free \\
\hline \hline
\end{tabular}

Coming back to the question of separating SM-like and non-SM-like $0^{+}$hypotheses, it is clear from Fig. 6.4 and Table 6.4 that the hypothesis $a_{1}=0$ and $a_{2} \neq 0$ in Eq. (4.1), corresponding to $f_{++}+f_{--}=0$, can be separated with high confidence 
Table 6.10: Results of the fit for the free parameters of the spin-two hypothesis with negative parity with generated samples of an exotic $X$ corresponding to $2^{-}$.

\begin{tabular}{|c|c|c|c|c|c|c|}
\hline & \multicolumn{3}{|c|}{$m_{X}=250 \mathrm{GeV}$} & \multicolumn{3}{|c|}{$m_{X}=1 \mathrm{TeV}$} \\
\hline & \multirow[t]{2}{*}{ generated } & \multicolumn{2}{|c|}{ fitted } & \multirow[t]{2}{*}{ generated } & \multicolumn{2}{|c|}{ fitted } \\
\hline & & without detector & with detector & & without detector & with detector \\
\hline$n_{\text {sig }}$ & 150 & $150 \pm 13$ & $152 \pm 15$ & 150 & $151 \pm 12$ & $152 \pm 13$ \\
\hline$f_{z 2}$ & 1.000 & $1.00 \pm 0.06$ & $0.97 \pm 0.12$ & 0.750 & $0.75 \pm 0.11$ & $0.81 \pm 0.15$ \\
\hline$f_{z 1}$ & 0.000 & $0.00 \pm 0.11$ & $0.05 \pm 0.20$ & 0.250 & $0.25 \pm 0.13$ & $0.19 \pm 0.24$ \\
\hline$f_{++}$ & 0.125 & $0.12 \pm 0.06$ & $0.13 \pm 0.07$ & 0.000 & $0.00 \pm 0.07$ & $0.00 \pm 0.07$ \\
\hline
\end{tabular}
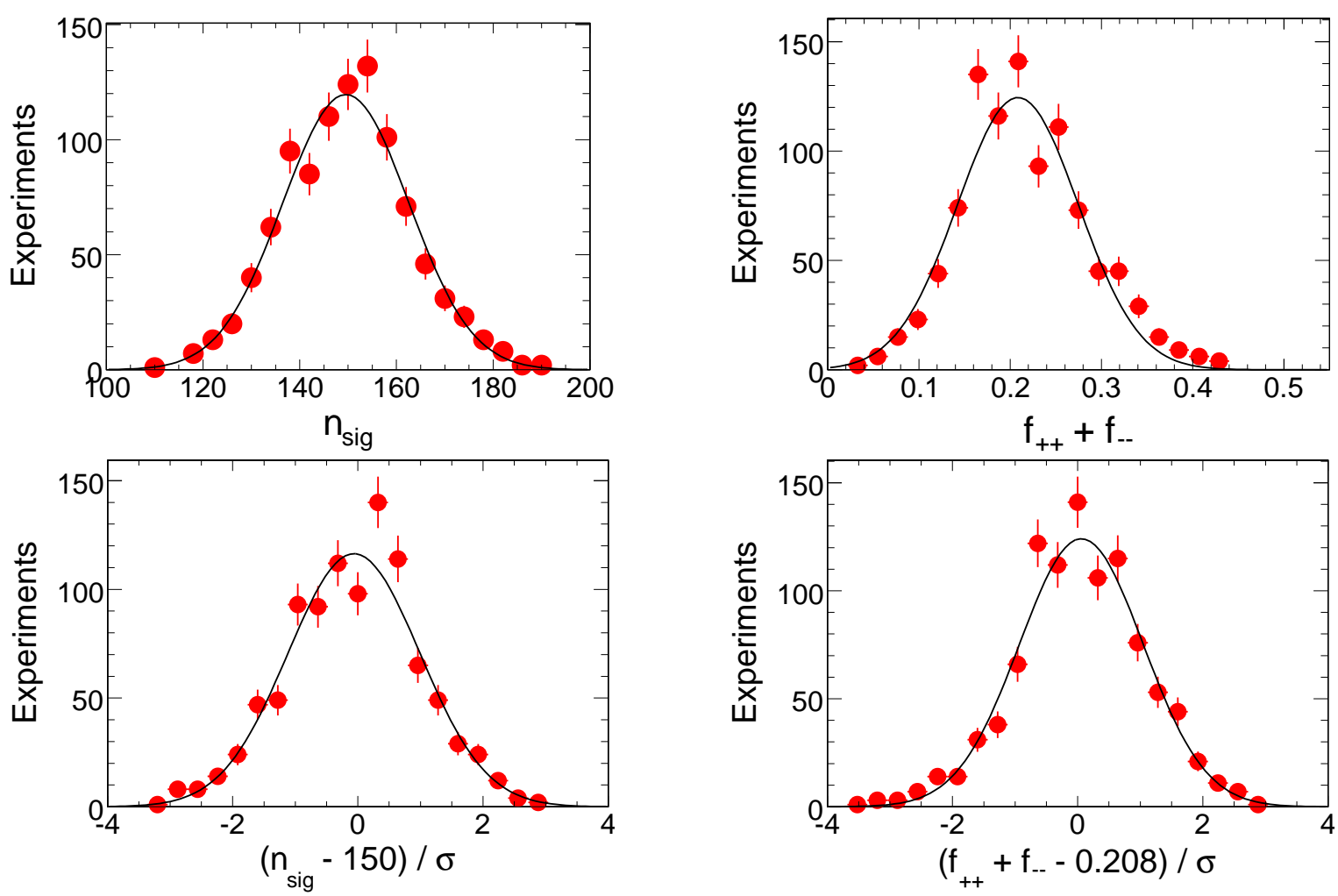

Figure 6.4: Fit for the parameters in Table 6.4 for a $0^{+}$signal hypothesis for 1000 toy experiments.

at $250 \mathrm{GeV}$, but no separation is possible at $1 \mathrm{TeV}$ due to the absence of a sizable transverse amplitude in both scenarios. As another example, the separation of the $1^{+}$ and $1^{-}$scenarios in Tables 6.2 and 6.3 is related to the measurement of $\left(\phi_{+0}-\phi_{0-}\right)$ in Tables 6.6 and 6.7, although there is an additional free parameter in the latter case. The signature of the minimal coupling model of a spin-two resonance decay is the dom- 
CHAPTER 6. STUDIES OF RESONANCES IN $X \rightarrow Z Z \rightarrow 4 \ell$

inance of the $A_{+-}$and $A_{-+}$amplitudes, while the model discussed in Refs. $[31,85,86]$ predicts longitudinal $A_{00}$ amplitude dominance. This was our motivation for comparing the $2_{m}^{+}$and $2_{L}^{+}$cases. We observe that the angular distributions of the two models could be well separated based on the measurement of $f_{+-}$, as it is also evident from Fig. 6.1 and Tables 6.2, 6.3. There are several cases where probability distributions of some parameter are not Gaussian; typically, this occurs when the central value of a parameter is at, or very close to, its physical boundary. In those cases, we still approximate the expectation value for this parameter and the error by $\mu \pm w$, where we extrapolate the value of $w$ from the rms, but we note that statistical meaning of the error estimated in such a way can be very different. For this reason, the proper interpretation of the values quoted in the tables in terms of the separation $\mathcal{S}$ value requires a more careful treatment which accounts for the non-Gaussian shape of all distributions.

The general conclusion from Tables $6.4-6.10$ is that our analysis yields unbiased results for all the parameters that describe the angular distributions in the production and decay of a resonance $X$. Indeed, it follows from those tables that, when detector effects are neglected, the central values of all the parameters correspond to their input values. This verifies both the fit implementation and angular parameterizations.

Once detector effects are introduced, certain biases appear but they are minor. These biases come from the fact that we ignore angular correlations in both the detector acceptance function and in our parametrization of angular background distri- 
CHAPTER 6. STUDIES OF RESONANCES IN $X \rightarrow Z Z \rightarrow 4 \ell$

butions. Both of these parameterizations are detector-specific and would need to be modeled in each experiment. For illustration purposes, we introduced a simplified model of the acceptance function in the present paper. We pointed out that the sophistication of the required model for the acceptance function and the background parametrization depends on the actual mass of the observed resonance. Indeed, it is evident from Tables $6.4-6.10$ that the bias is smaller at higher masses due to reduced angular correlations, as we explained earlier. The expected statistical errors of the measurements are not strongly affected by detector effects; this allows us to predict statistical precision in future measurements.

\section{Data Analysis with CMS Simulation}

A subset of the tests performed above with standalone detector simulation is performed using a full CMS simulation. The Monte Carlo program is interfaced by a specific LHE format to Pythia using the CMS software. Then those events are used as input to the full CMS GEANT simulation. These studies are performed with 20 signal events and a number of background events corresponding to approximately $5 \mathrm{fb}^{-1}$ of

data at the LHC. The center of mass energy of the LHC is assumed to be $\sqrt{s_{p p}}=7 \mathrm{TeV}$. Tables $6.11,6.12,6.13$. The tables show separation for $m_{X}=250,500,1000 \mathrm{GeV}$, respectively. The studies with full CMS simulation do not exactly match those studies done with standalone simulation, but the confidence level with which we can separate signal hypotheses are summarized these tables. Finally, in Table 6.14, we also present 
CHAPTER 6 . STUDIES OF RESONANCES IN $X \rightarrow Z Z \rightarrow 4 \ell$

a first separation of the $0^{+}$and $0^{-}$hypotheses at a $m_{X}=140 \mathrm{GeV}$ below the $Z Z$ threshold. This separation uses information about the off-shell $Z$ mass distribution in addition to the usual angular distributions. This can be seen in Fig. 6.5 where we have illustrated the off-shell $Z$ mass distribution in three cases: a scalar resonance with a $140 \mathrm{GeV}$ mass (left), a pseudoscalar resonances with a $140 \mathrm{GeV}$ mass (middle) and the $Z Z$ background (right).

Table 6.11: Results of the hypothesis separation $(\mathcal{S})$ of the seven scenarios for $m_{X}=$ $250 \mathrm{GeV}$. with the $X \rightarrow Z Z \rightarrow 4 l$.

\begin{tabular}{lcccccc}
\hline \hline & $0^{-}$ & $1^{+}$ & $1^{-}$ & $2_{m}^{+}$ & $2_{L}^{+}$ & $2^{-}$ \\
\hline $0^{+}$ & 3.3 & 1.8 & 2.0 & 2.2 & 1.5 & 2.7 \\
$0^{-}$ & & 2.6 & 2.4 & 1.7 & 3.9 & 2.2 \\
$1^{+}$ & & & 1.7 & 1.7 & 2.4 & 2.1 \\
$1^{-}$ & & & & 1.1 & 2.5 & 2.4 \\
$2_{m}^{+}$ & & & & & 2.8 & 2.1 \\
$2_{L}^{+}$ & & & & & & 3.3 \\
\hline \hline
\end{tabular}

Table 6.12: Results of the hypothesis separation $(\mathcal{S})$ of the seven scenarios for $m_{X}=$ $500 \mathrm{GeV}$. with the $X \rightarrow Z Z \rightarrow 4 l$.

\begin{tabular}{lcccccc}
\hline \hline & $0^{-}$ & $1^{+}$ & $1^{-}$ & $2_{m}^{+}$ & $2_{L}^{+}$ & $2^{-}$ \\
\hline $0^{+}$ & 4.3 & 2.6 & 2.9 & 3.6 & 1.3 & 2.7 \\
$0^{-}$ & & 2.7 & 2.6 & 1.9 & 4.6 & 2.8 \\
$1^{+}$ & & & 1.9 & 1.9 & 3.1 & 1.9 \\
$1^{-}$ & & & & 1.8 & 3.2 & 2.1 \\
$2_{m}^{+}$ & & & & & 4.0 & 2.7 \\
$2_{L}^{+}$ & & & & & & 3.0 \\
\hline \hline
\end{tabular}


CHAPTER 6. STUDIES OF RESONANCES IN $X \rightarrow Z Z \rightarrow 4 \ell$

Table 6.13: Results of the hypothesis separation $(\mathcal{S})$ of the seven scenarios for $m_{X}=1$ $\mathrm{TeV}$ with the $X \rightarrow Z Z \rightarrow 4 l$.

\begin{tabular}{ccccccc}
\hline \hline & $0^{-}$ & $1^{+}$ & $1^{-}$ & $2_{m}^{+}$ & $2_{L}^{+}$ & $2^{-}$ \\
\hline $0^{+}$ & 4.7 & 2.9 & 2.9 & 4.1 & 0.8 & 2.9 \\
$0^{-}$ & & 2.8 & 2.8 & 1.9 & 4.4 & 2.7 \\
$1^{+}$ & & & 2.0 & 2.0 & 3.0 & 2.0 \\
$1^{-}$ & & & & 1.9 & 2.8 & 2.2 \\
$2_{m}^{+}$ & & & & & 3.9 & 2.6 \\
$2_{L}^{+}$ & & & & & & 2.8 \\
\hline \hline
\end{tabular}
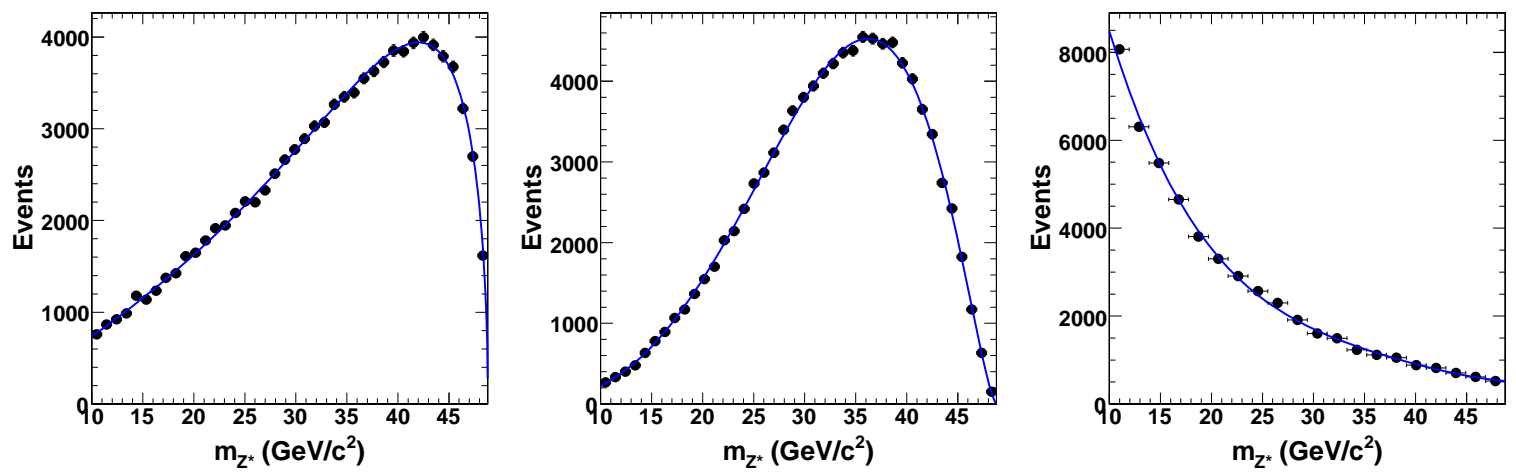

Figure 6.5: Projection off-shell $Z$ mass for three samples at $140 \mathrm{GeV}$ : signal $0^{+}$, signal $0^{-}$, and background.

Table 6.14: Results of the hypothesis separation of the $0^{+}$and $0^{-}$scenarios for $m_{X}=140 \mathrm{GeV}$.

\begin{tabular}{ll}
\hline \hline & $0^{-}$ \\
\hline $0^{+}$ & 3.3 \\
\hline
\end{tabular}

\section{Outlook on resonance properties}

The importance of the LHC physics program for the future of high-energy physics requires designing techniques and analysis tools that are not biased towards a particular model of beyond the Standard Model physics. While it may be impractical or impossible to do so in many complicated cases, the case of a single resonance produc- 
CHAPTER 6. STUDIES OF RESONANCES IN $X \rightarrow Z Z \rightarrow 4 \ell$

tion at the LHC is sufficiently robust to aim at the most general description, yet simple enough to make such a description possible. Motivated by this, we studied production of a single resonance at the LHC and its decay into four-lepton final states in the process $p p \rightarrow X \rightarrow Z Z \rightarrow \ell_{1} \bar{\ell}_{1} \ell_{2} \bar{\ell}_{2}$, allowing for the most general couplings of the resonance to Standard Model matter and gauge fields. Our goal was to understand if full reconstruction of the final state and the analysis of the most general angular distributions can be used to determine the spin and parity of the resonance and to constrain its couplings to vector gauge bosons, quarks, and gluons.

We used those angular distributions to construct the likelihood functions for the angular analysis. We wrote a Monte Carlo simulation program that describes the production of the resonance $X$ in the process $p p \rightarrow X \rightarrow Z Z \rightarrow \ell_{1} \bar{\ell}_{1} \ell_{2} \bar{\ell}_{2}$, includes all spin correlations throughout the decay chains, and employs the most general couplings of the resonance $X$ to matter and gauge fields of the Standard Model. We supplemented this analysis with a simplified (but fairly realistic) model of the detector effects. When all the pieces of our study are put together, we obtain a powerful analysis tool that allows for a realistic estimate of how much information the two LHC experiments will be able to extract from the study of angular distributions once the resonance in the process $p p \rightarrow X \rightarrow Z Z \rightarrow \ell_{1} \bar{\ell}_{1} \ell_{2} \bar{\ell}_{2}$ is observed.

We find that angular distributions provide good separation of various hypotheses about the spin of the resonance. As we illustrate in Tables 6.2 and 6.3, with 30 fully reconstructed events a typical separation of various spin hypotheses is $\mathcal{S}=2-3 \sigma$, 
CHAPTER 6. STUDIES OF RESONANCES IN $X \rightarrow Z Z \rightarrow 4 \ell$

as defined in text, but in some cases separation as good as $4 \sigma$ is achieved. From Tables 6.2 and 6.3 , it is clear that even with $\mathrm{O}(30)$ events, at the time of the discovery of a new resonance, we are already able to make a statement about the nature of the discovered resonance.

With a somewhat larger event sample, it becomes possible to determine helicity amplitude fractions that characterize resonance production and decay as well as the resonance spin from a multidimensional likelihood fit with decent precision. Modelindependent determination of the helicity amplitude fractions and phases is the ultimate goal of such an analysis which can then be interpreted within any model of beyond the Standard Model physics. We provide relationships between the amplitude measurements and the fundamental coupling constants of the resonance to matter and gauge fields. Our studies show that such model-independent analysis is viable at the LHC. We look forward to its application to real LHC data.

Finally, we note that the analysis presented in this paper can be extended in a number of ways. A natural possibility is to allow hadronic final states (e.g. $Z \rightarrow q \bar{q}$ ) and/or missing energy. This has been explored in greater detail at CMS in the search for a SM Higgs decaying to $Z Z \rightarrow 2 \ell 2 q$. Another obvious candidate for the latter is the decay $X \rightarrow W^{+} W^{-}$. We note that most of the discussion of the $X \rightarrow Z Z$ decays given in this paper also applies to decays $X \rightarrow W^{+} W^{-}$. However, there are important differences related to the fact that $W^{+}$and $W^{-}$are not identical particles. Consequently, Eq. 4.12 does not apply in the $W^{+} W^{-}$case and nine, rather than six, 
CHAPTER 6. STUDIES OF RESONANCES IN $X \rightarrow Z Z \rightarrow 4 \ell$

independent helicity amplitudes are required to describe $X \rightarrow W^{+} W^{-}$decay. While the increase in the number of the helicity amplitudes implies that most of the formulas for angular distributions derived in this paper are not complete for $X \rightarrow W^{+} W^{-}$, it is interesting to remark that Eq. 4.1 remains the most general description of a spin-zero $X \rightarrow W^{+} W^{-}$decay. Another aspect of the decay of the resonance $X$ to two nonidentical particles is that forward-backward asymmetry can be generated. Of course, we have looked at this in great detail with the SM Drell-Yan process in Chapter 5. On the experimental side, the angular analysis of $X \rightarrow W^{+} W^{-} \rightarrow 4 l$ decay is challenging due to the presence of two neutrinos in the final state. It might be beneficial to consider semileptonic final states where one $W$ decays hadronically and the other leptonically. However, this case requires detailed studies because of potentially large irreducible backgrounds from events with jets and missing energy. Similar issues should be investigated if semileptonic final states are allowed in $X \rightarrow Z Z$ decays. We hope to return to the discussion of these channels in the near future.

These methods may also be used in direct searches for $X \rightarrow Z Z \rightarrow 4 l$ resonances where we can use the angular distributions to separate the background from the signal. Our sensitivity for SM Higgs searches can be improved by using the angular information to discriminate between the signal and background hypotheses. In particular, this is also true below the $Z Z$ invariant mass threshold. The formalism has been developed to describe resonances below a mass of $2 m_{Z}$ where there is also dependence on the off-shell $Z$ mass. Combining the mass information with the angles 
CHAPTER 6. STUDIES OF RESONANCES IN $X \rightarrow Z Z \rightarrow 4 \ell$

into a single likelihood function will provide the best discriminating power between a signal resonance and the background in this mass region.

\subsection{Search for the Higgs in $H \rightarrow Z Z \rightarrow 4 \ell$}

As the integrated luminosity at the LHC grows, we begin to study the $X \rightarrow Z Z \rightarrow$ $4 l$ process in greater detail. While the current luminosity of $1.66 \mathrm{fb}^{-1}$ may not yet be ideal for performing angular analysis, a search is performed for the SM Higgs boson. The search covers the mass range from 110 to $600 \mathrm{GeV}$. The direct searches for the SM Higgs boson at the LEP $\mathrm{e}^{+} \mathrm{e}^{-}$collider have lead to a lower mass bound of $m_{\mathrm{H}}>114.4 \mathrm{GeV}$ at $95 \%$ confidence level $(95 \% \mathrm{CL})$ [5]. The direct searches by the D0 and CDF experiments at the Tevatron exclude the mass range $158<m_{\mathrm{H}}<173 \mathrm{GeV}$ (95\% CL) [87]. The mass of the SM Higgs is favored to be in the range $m_{\mathrm{H}}<185 \mathrm{GeV}$ due to electroweak precision measurements [88]. In the absence of new physics beyond the SM, the requirement of perturbative unitarity of the theory sets an upper bound on $m_{\mathrm{H}}$ in a range from about 500 to $800 \mathrm{GeV}$ [89-91].

In this section, a brief description of this search for the Higgs boson in the channel $p p \rightarrow H \rightarrow Z Z^{(*)} \rightarrow \ell^{+} \ell^{-} \ell^{+} \ell^{-}$is given, while full details can be found in [83]. We emphasize the statistical interpretations and results of this search and present the exclusion limits. The intention is to present the latest results from the CMS experiment in this channel and provide an up-to-date look at the status of this decay mode at 
CHAPTER 6. STUDIES OF RESONANCES IN $X \rightarrow Z Z \rightarrow 4 \ell$

the LHC. The SM Higgs cross-section at the LHC at $\sqrt{s_{p p}}=7 \mathrm{TeV}$ is provided [92] in Fig. 6.6. The leading production mechanism at the LHC for the Higgs is gluon fusion $p(g) p(g) \rightarrow H$ where the Feynam diagram for this process is similar to the middle diagram in Fig. 4.2. In addition, there are other non-negligible production contributions from weak-boson fusion ( $p p \rightarrow q q H)$, vector boson associated production $(p p \rightarrow W H$ and $p p \rightarrow Z H)$ and $t \bar{t}$ associated production $(p p \rightarrow t \bar{t} H)$. The branching ratios of the SM Higgs are provided [92] in Fig. 6.7. Of particular note is the branching ratio of the Higgs to $Z Z$ where the decay becomes significant above the $Z Z$ mass threshold. The shape of this branching ratio gives us a feeling for the sensitivity of this channel where in the range $m_{H} \sim[140,160] \mathrm{GeV}$ the $Z Z$ channel also has a significant contribution.

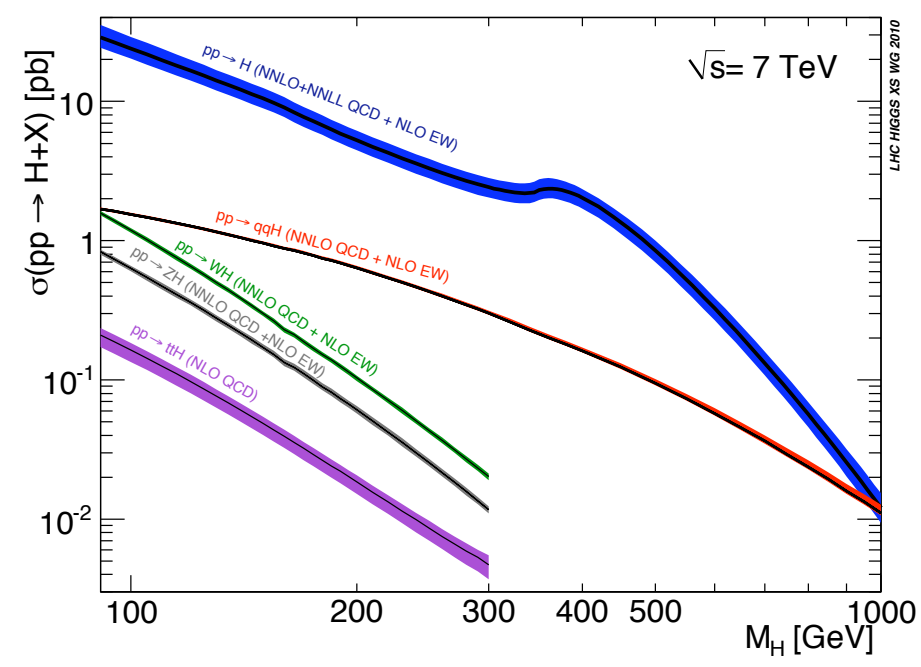

Figure 6.6: Production cross-section for SM Higgs at $7 \mathrm{TeV}$ 
CHAPTER 6. STUDIES OF RESONANCES IN $X \rightarrow Z Z \rightarrow 4 \ell$

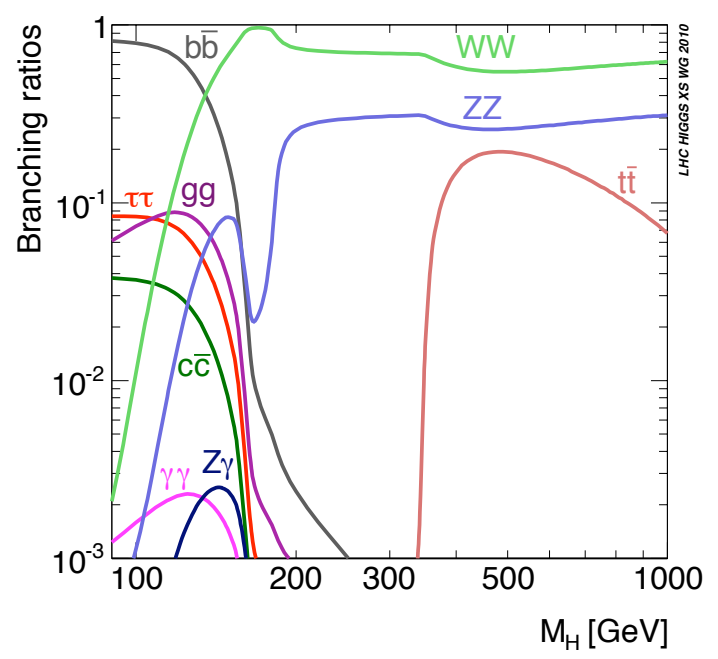

Figure 6.7: Branching ratios for SM Higgs at $7 \mathrm{TeV}$

\section{Datasets, Event Selection, and Backgrounds}

The CMS data sample of $1.66 \pm 0.07 \mathrm{fb}^{-1}$ used for this study is based on data collected in 2010 and 2011. Monte Carlo (MC) data samples for the SM Higgs boson signal and a large variety of electroweak and QCD-induced SM background processes, including full simulation and reconstruction, are used for the optimization of the event selection. Cross sections are re-weighted at least to next-to-leading-order (NLO), or beyond where possible. The general multi-purpose MC event generator PYTHIA [93] is used in conjunction with other event generators, for the showering, hadronization, decays and to add the underlying pp event. The events are processed with a detailed simulation of the CMS detector based on GEANT4 [94]. The Higgs boson samples are generated with POWHEG [95] which incorporates NLO gluon fusion 
CHAPTER 6. STUDIES OF RESONANCES IN $X \rightarrow Z Z \rightarrow 4 \ell$

$(g g \rightarrow \mathrm{H})$ and weak-boson fusion $q \bar{q} \rightarrow q \bar{q} \mathrm{H}$. The event rates are scaled according to the most recent calculations [92] of the total cross section $\sigma(p p \rightarrow \mathrm{H})$. The total cross section is scaled by the branching ratio $\mathcal{B}(\mathrm{H} \rightarrow 4 \ell)[92,96-99]$ where interference in the $4 e$ or $4 \mu$ channels is taken into account using the Prophecy $4 \mathrm{f}[92,96,97]$ generator tool for precision calculations. Di-boson production (WW, WZ, ZZ, Z $\gamma$ ) is generated at leading order (LO) with PYTHIA. The leading background contribution from $\mathrm{ZZ}^{(*)}$ events are re-weighted with a $m_{4 \ell}$-dependent $\mathrm{K}$-factor to account for the contributions of NLO diagrams. Both $q \bar{q} \rightarrow \mathrm{ZZ}^{(*)}$ and $g g \rightarrow \mathrm{ZZ}^{(*)}$ contributions are evaluated with MCFM [100]. An uncertainty of $\pm 30 \%$ is attributed to the gluon-induced contribution. An inclusive Z+jets sample has been generated with MadGraph [101] at LO and corrected with a NLO k-factor, including decays to gluons and light $(d, u, s)$ and heavy $(c, b)$ quarks for the jets in the final state. This sample is split at generator level into separate contributions of $Z+$ light jets ("Z+jets") and $Z+$ heavy flavour jets $(\mathrm{Z} b \bar{b} / \mathrm{Z} c \bar{c})$ for more detailed study. Top pair production is generated at NLO with POWHEG.

The trigger used in this analysis has evolved in response to the rapidly increasing instantaneous luminosity. The presence of at least one charged lepton, either an electron or a muon with a $p_{T}^{\ell}$ above $\mathcal{O}(10) \mathrm{GeV}$, is required for the sample corresponding to the early data taking with instantaneous luminosities in the range $10^{29}-10^{31} \mathrm{~cm}^{-2} \mathrm{~s}^{-1}$. The presence of a pair of electrons with $E_{T, 1}^{e}>17$ and $E_{T, 2}^{e}>8 \mathrm{GeV}$, or a pair of muons with $p_{T, 1}^{\mu}>13$ and $p_{T, 2}^{\mu}>8 \mathrm{GeV}$, is required for a major fraction of the data 
CHAPTER 6. STUDIES OF RESONANCES IN $X \rightarrow Z Z \rightarrow 4 \ell$

sample, collected with instantaneous luminosities up to $\simeq 2.2 \times 10^{33} \mathrm{~cm}^{-2} \mathrm{~s}^{-1}$. The trigger is virtually fully efficient $(\geq 99 \%)$ for the $4 e, 4 \mu$ and $2 e 2 \mu$ channels. Standard CMS reconstruction of the muons is detailed in Section 5.2. A detailed description of the electron reconstruction is outlined in Ref. [83].

Due to the low amount of statistics expected at the current luminosity, about 20 events, and the relatively clean nature of the $4 l$ final state, the analysis strategy is to keep kinematic requirements sufficiently loose to preserve the high trigger efficiency. There are loose selections made on the masses of the first and second $Z$ and a requirement of four or more leptons. The $p_{T}$ requirements on the leptons are kept loose and are only required to be consistent with trigger requirements. Then, additional cuts are meant to supress reducible background from $Z+$ jets, $Z b \bar{b} / Z c \bar{c}$ and $t \bar{t}$. A requirement is made on the lepton isolation in order to ensure that the leptons do not original from jets. There is also a selection based on the impact parameter of the leptons such that they come from the same vertex. This is necessary to reduce backgrounds where leptons originate from displaced vertices. After requiring all selection requirements, the remaining dominant background contribution comes from the irreducible $Z Z \rightarrow 4 l$ process while only smaller contributions from $Z+$ jets, $Z b \bar{b} / Z c \bar{c}$ and $t \bar{t}$ remain.

The small number of events observed makes it difficult to estimate the background using mass sidebands. Such an approach will have large statistical uncertainties and is only feasible at higher integrated luminosities. Instead the procedure is to choose a wide background control region outside the signal phase space which is populated 
CHAPTER 6. STUDIES OF RESONANCES IN $X \rightarrow Z Z \rightarrow 4 \ell$

by relaxing some selection criteria, and verify that the event rates change according to the MC expectation.

The $Z Z$ background contribution is normalized to the $Z$ inclusive cross-section. The ratio of the $Z Z$ and $Z$ cross-sections are examined in Monte Carlo simulation. Then this ratio is used to scale the $Z$ cross-section measurement made in data to the expected $Z Z$ cross-section. Care is required with the acceptance cuts to ensure an accurate ratio determination. A cross-check is performed by comparing the event rate from this normalization with an estimate purely from simulation. The background yields for the $Z Z$ contribution is presented in Table 6.15. In the case of the reducible $Z+$ jets, $Z b \bar{b} / Z c \bar{c}$ and $t \bar{t}$ backgrounds, the control region is defined by relaxing the isolation and impact parameter requirements. Then an extrapolation is made from this control region to the signal region where the scale factor is taken from simulation. A summary of the expected background rates for these reducible background contributions are given in Table 6.16 .

Table 6.15: Number of $Z Z$ background events from $m_{4 l}=$ [100-600] GeV for two methods of estimation

\begin{tabular}{c|cc}
\hline channel & Normalization to Z rate & MC model simulation \\
\hline$N^{\mathrm{ZZ} \rightarrow 4 e}$ & $4.05 \pm 0.26$ & $4.07 \pm 0.38$ \\
$N^{\mathrm{ZZ} \rightarrow 4 \mu}$ & $6.02 \pm 0.40$ & $6.23 \pm 0.57$ \\
$N^{\mathrm{ZZ} \rightarrow 2 e 2 \mu}$ & $9.87 \pm 0.66$ & $10.06 \pm 0.93$ \\
\hline
\end{tabular}


CHAPTER 6. STUDIES OF RESONANCES IN $X \rightarrow Z Z \rightarrow 4 \ell$

Table 6.16: Number of expected events and relative uncertainties for reducible for reducible background contributions $t \bar{t}, Z b \bar{b} / c \bar{c}$ and $Z+$ jets

\begin{tabular}{|c|c|}
\hline$N^{\mathrm{Z} b \bar{b} / c \bar{c}, t \bar{t} \rightarrow 4 e}$ & $0.01 \pm 0.01$ \\
$N^{\mathrm{Z} b \bar{b} / c \bar{c}, t \bar{t} \rightarrow 4 \mu}$ & $0.05 \pm 0.01$ \\
$N^{\mathrm{Z} b \bar{b} / c \bar{c}, t \bar{t} \rightarrow 2 e 2 \mu}$ & $0.06 \pm 0.01$ \\
\hline$N^{\mathrm{Z}+\text { jets } \rightarrow 4 e}$ & $0.48 \pm 0.08$ \\
$N^{\mathrm{Z}+\text { jets } \rightarrow 4 \mu}$ & $0.09 \pm 0.02$ \\
$N^{\mathrm{Z}+\text { jets } \rightarrow 2 e 2 \mu}$ & $0.61 \pm 0.11$ \\
\hline
\end{tabular}

\section{Systematics}

The main sources of systematic uncertainties on the expected yields are summarized in Table 6.17. Systematic uncertainties have been evaluated from data for the trigger efficiency as well as for effects from individual lepton reconstruction, identification and isolation efficiencies, and from energy-momentum calibration. Additional systematic uncertainties affecting the derivation of exclusion limits come from the limited statistics in the background control regions which propagate to the background evaluation in the signal region. All major background sources are derived from control regions, and the comparison of the data with the background expectation in the signal region is independent of the uncertainty on the LHC integrated luminosity for the data sample. This uncertainty enters in the calculation of a cross section limit through the normalization of the signal. Also given for completeness in Table 6.17 is 
CHAPTER 6. STUDIES OF RESONANCES IN $X \rightarrow Z Z \rightarrow 4 \ell$

an evaluation of the systematics uncertainties on the Higgs boson cross section and branching ratio [92].

Table 6.17: Summary of the magnitude of systematic uncertainties in percent.

\begin{tabular}{c|c}
\hline Luminosity & 4.5 \\
Trigger efficiency & 1.5 \\
Higgs cross section & $17-20$ \\
Higgs B.R. & 2 \\
Lepton reco/ID eff. & $2-3$ \\
Lepton isolation eff. & 2 \\
Electron energy scale & 3 \\
\hline
\end{tabular}

\section{Statistical Interpretation and Results}

In order to quantify the sensitivity of the experiment to the presence of a Higgs boson signal, an the unbinned one-dimensional $m_{4 l}$ distribution is used as a discriminator.

We use the $C L_{s}$ method or hybrid frequentist-bayesian [102] to quantify the upper limit on the SM Higgs boson signal. A likelihood function is constructed from the expected signal and background distributions of the four lepton invariant mass. Systematic uncertainties enter the likelihood as multiplicative corrections to the yields. 
CHAPTER 6. STUDIES OF RESONANCES IN $X \rightarrow Z Z \rightarrow 4 \ell$

For each value of the signal strength $r$ defined as the ratio of the signal cross-section to the SM Higgs cross-section $\sigma / \sigma_{S} M$, a test statistic is defined starting from the likelihood ratio $2 \ln Q=2 \ln \left(\mathcal{L}_{s+b}(r) / \mathcal{L}_{b}\right)$ where $\mathcal{L}_{s+b}(r)$ is likelihood for the signal-plusbackground hypothesis, and $\mathcal{L}_{b}$ is the one for the background-only hypothesis. The predicted distribution of the test statistic in the two cases is determined from toy Monte Carlo, performing a bayesian pseudo-integration on the systematical uncertainties. The observed value of the test statistic is then compared with the prediction, yielding the $p$-values given the background or the signal-plus-background hypotheses. The two $p$-values are combined to construct the pseudo $p$-value $C L_{s}=C L_{s+b} /\left(1-C L_{b}\right)$. The upper limit on $r$ at $95 \%$ confidence level is defined as the value of $r$ for which $C L_{s}=0.05$.

\section{Inputs to the Exclusion Limits}

The exclusion limits for a SM-like Higgs Boson are computed for a large number of mass points in the mass range from 110 to $600 \mathrm{GeV}$. The choice of the intervals in between Higgs mass hypotheses is driven by either detector resolution of the resonance or its natural width depending on which is larger. For the masses from 110 -160 GeV, the limits are computed every $1 \mathrm{GeV}$; for the masses from 160-290 GeV, the limits are computed every $2 \mathrm{GeV}$; for the masses from 290-350 GeV, the limits are computed every $5 \mathrm{GeV}$; for the masses from $350-400 \mathrm{GeV}$, the limits are computed every $10 \mathrm{GeV}$; for the masses from $400-600 \mathrm{GeV}$, the limits are computed every $20 \mathrm{GeV}$. The 
CHAPTER 6. STUDIES OF RESONANCES IN $X \rightarrow Z Z \rightarrow 4 \ell$

choice of Higgs mass points allows for good sensitivity of the exclusion limits in the full mass range. Due to the large number of mass points and availability of simulated signal samples, we must interpolate the mass shapes of signal hypotheses where no simulation exists.

As discussed above, we determine the exclusion limits using the mass shape method. The mass shape method uses the unbinned $4 l$ invariant mass distribution for the hypothesized signal shape and expected background shape as a discriminator. Because of the mass shape interpolation, we determine the shapes of the hypothesized signal and expected background distributions. With these shapes we may perform the calculation of the exclusion limits via the mass shape method.

As discussed in previous sections, the contributing background processes consist of the irreducible electroweak $Z Z$ production and reducible contributions coming from $Z+$ jets, $Z b \bar{b}$, and $t \bar{t}$. The shape of the irreducible $Z Z$ background contributions are determined fitting for the shape of the leading-order (LO) $4 l$ invariant mass distribution in simulation and then folding in the next-to-leading-order (NLO) effects from both $q \bar{q} \rightarrow Z Z$ and $g g \rightarrow Z Z$ processes. The LO mass distribution is fit with the empirical functional form [103]:

$$
\frac{d N}{d m}=f_{1} \times F(m, \hat{x})+\left(1-f_{1}\right) \times F(m, \hat{y})
$$

where

$$
F(m, \hat{a})=\left(0.5+0.5 \times \operatorname{sign}\left(m-a_{1}\right) \times \operatorname{erf}\left(\frac{\left|m-a_{1}\right|}{a_{2}}\right) \times \exp \left(\frac{m}{a_{3}}\right)\right.
$$


CHAPTER 6. STUDIES OF RESONANCES IN $X \rightarrow Z Z \rightarrow 4 \ell$

In Fig. 6.8, we plot the simulation and the fit function for the $2 e 2 m u$ (left), $4 e$ (middle) and $4 \mu$ (right) decay channels. The fit and simulation show good agreement. The
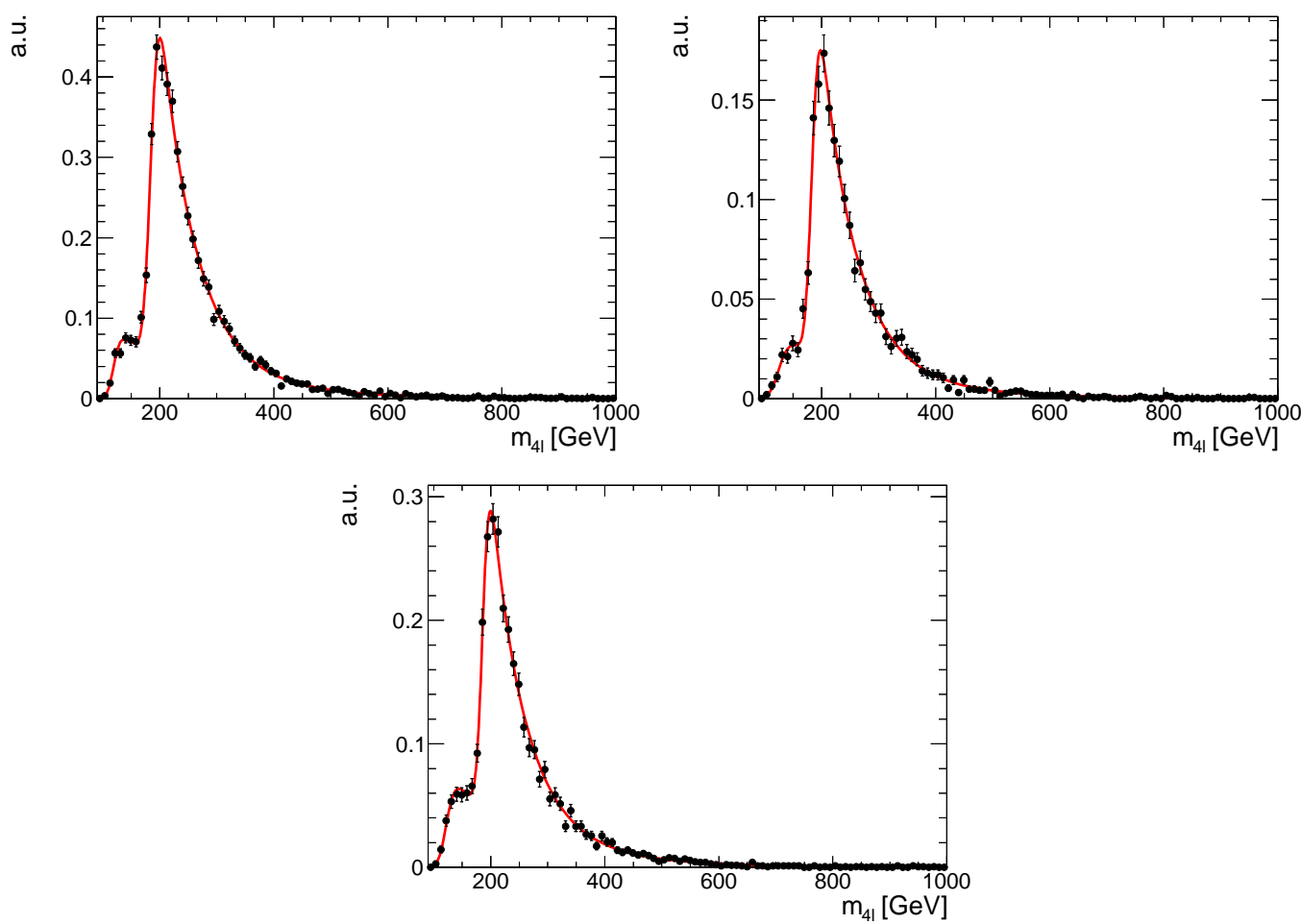

Figure 6.8: Leading-order invariant mass distribution for the $Z Z$ background contribution (points) and the fitted PDF (lines).

NLO effects from $Z Z$ irreducible background processes are shown as a function of $4 l$ invariant mass in Fig. 6.9. They are calculated using the MCFM Monte Carlo program.

The shapes for the reducible backgrounds are also determined from simulation. The shapes are parameterized with Landau functions. The yields for the reducible backgrounds are determined from data-driven techniques extrapolating from a control region into the signal region and contribute mostly at lower Higgs mass hypotheses. 
CHAPTER 6. STUDIES OF RESONANCES IN $X \rightarrow Z Z \rightarrow 4 \ell$
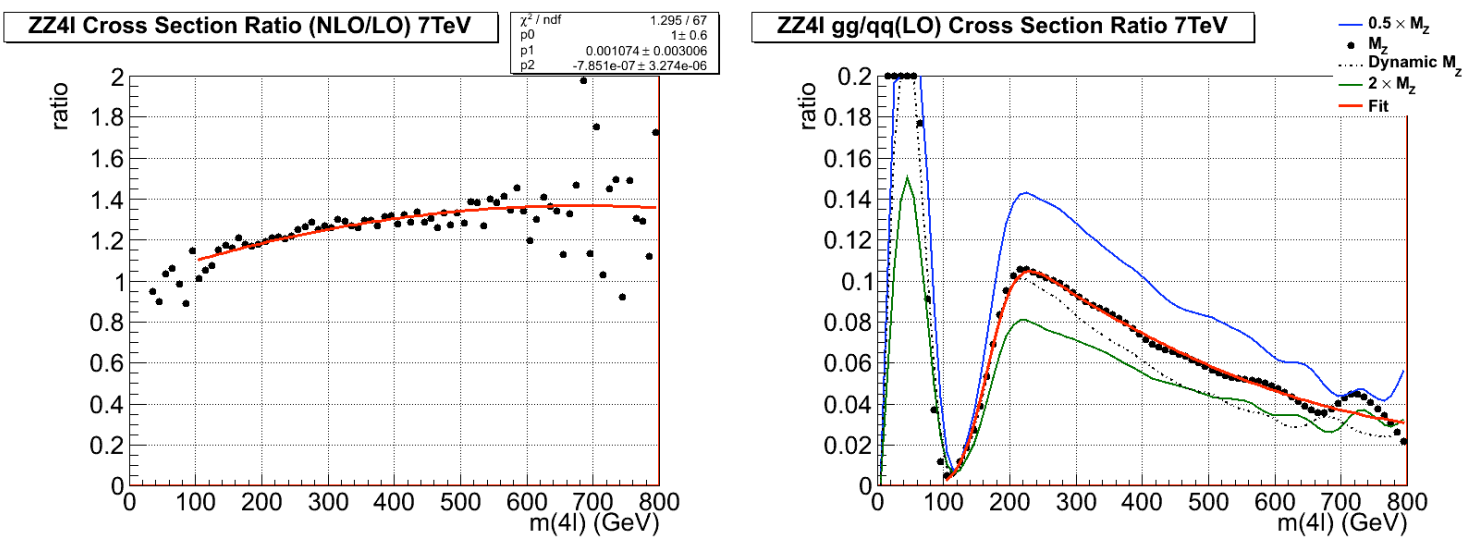

Figure 6.9: NLO contributions for the $Z Z$ backgrounds for $q q \rightarrow Z Z$ (left) and $g g \rightarrow$ $Z Z$ (right)

The signal shape is determined using 17 simulated POWHEG samples over the full mass range. We fit for the shapes for each simulated sample using a Breit-Wigner-like PDF to describe the theoretical resonance line shape convoluted with a Crystal-Ball function to describe the detector effects. Then we interpolate the parameters of the Crystal-Ball function to the Higgs mass points where there is no simulated sample available.

The Higgs theoretical mass distribution is described by the functional form [104]:

$$
B W\left(m_{4 l} \mid m_{H}\right)=\frac{1}{\mathcal{N}} \frac{\Gamma_{g g}\left(m_{4 l}\right) \Gamma_{Z Z}\left(m_{4 l}\right)}{\left(m_{4 l}^{2}-m_{H}^{2}\right)^{2}+m_{4 l}^{2} \Gamma_{\text {tot }}\left(m_{4 l}\right)} * L_{g g}^{\prime}\left(m_{4 l}\right) * m_{4 l}
$$

Here, $m_{4 l}$ is the $4 l$ invariant mass while $m_{H}$ is the mass of the Higgs hypothesis. The partial Higgs widths [92] are given by $\Gamma$ and $L_{g g}^{\prime}\left(m_{4 l}\right)$ is the gluon partonic luminosity function, an example of which is given in Fig. 4.1. The motivation for choosing the shape is to cover the mass distribution in the full search region. In particular, the running width and gluon partonic luminosity function is important in the high mass 
CHAPTER 6. STUDIES OF RESONANCES IN $X \rightarrow Z Z \rightarrow 4 \ell$

region above approximately $300 \mathrm{GeV}$. Further, the choice of signal theoretical mass distribution is flexible enough to encompass theoretical uncertainties on the line shape in the high mass region. We then convolute this line shape with a Crystal-Ball function to perform the final signal fit. The fits for a few representative mass points are given in Fig. 6.10. The $2 \mathrm{e} 2 \mu$ channel is on the left, $4 \mathrm{e}$ channel in the middle, and $4 \mu$ channel on the right. The $120 \mathrm{GeV}$ mass point is on top, $200 \mathrm{GeV}$ mass point is in the middle, and $400 \mathrm{GeV}$ mass point is on the bottom. We then interpolate the parameters of
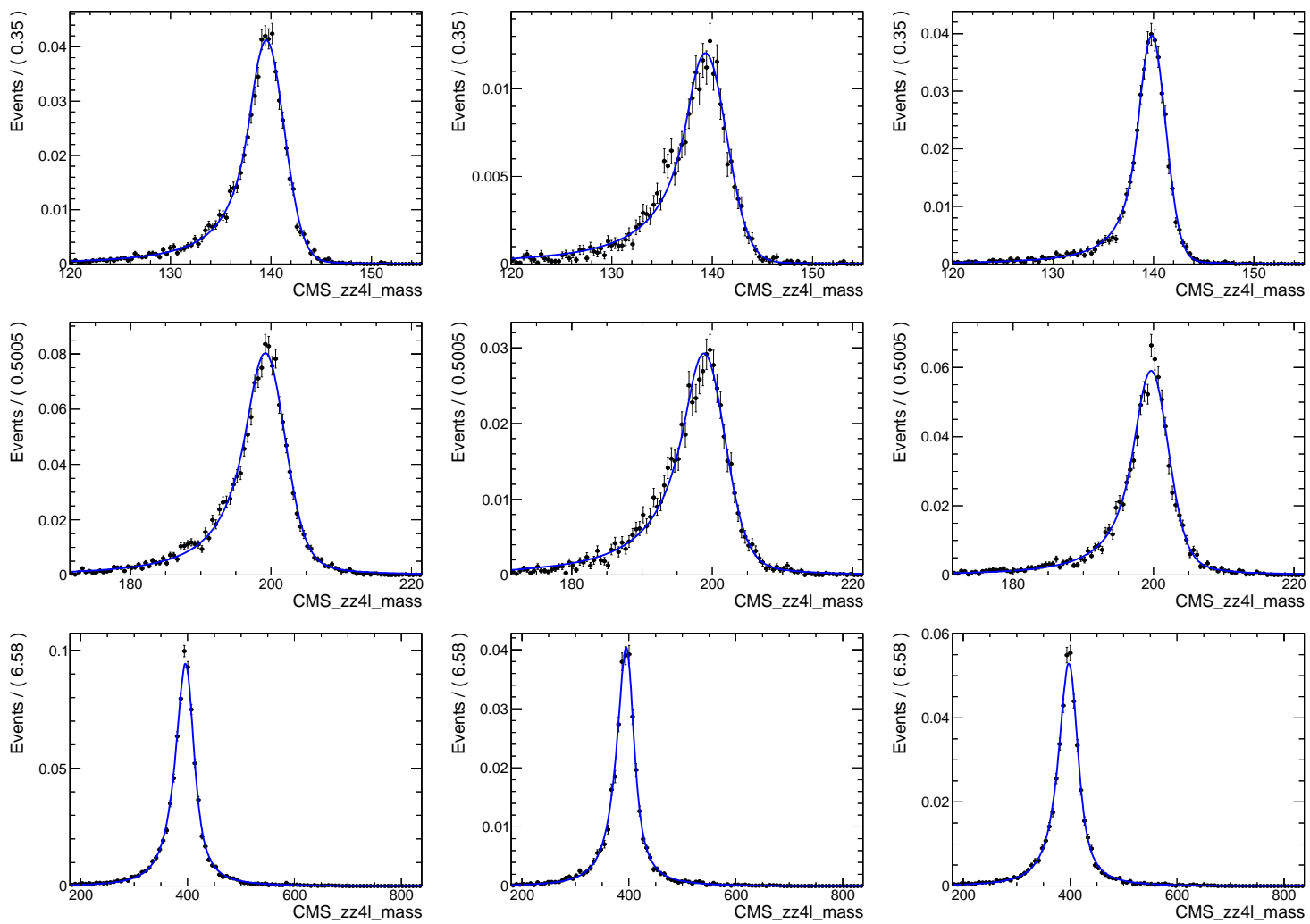

Figure 6.10: Fits of simulated Higgs signal events for three different mass points

the signal mass distribution at mass points where no simulated sample is available. 
CHAPTER 6. STUDIES OF RESONANCES IN $X \rightarrow Z Z \rightarrow 4 \ell$

The procedure for this in the $2 e 2 \mu$ channel is shown in Fig. 6.11. The Breit-Wigner function parameters $m_{H}$ (top left) and $\Gamma_{H}$ (top middle) are fixed to their theoretical values. The tail parameters of the Crystal Ball function $\alpha$ (bottom middle) and $n$ (bottom right) are fixed based on the radiative tail and are different for the 3 channels. The mean (top right) and width (bottom left) of the Crystal ball function are left to float as a function of mass.
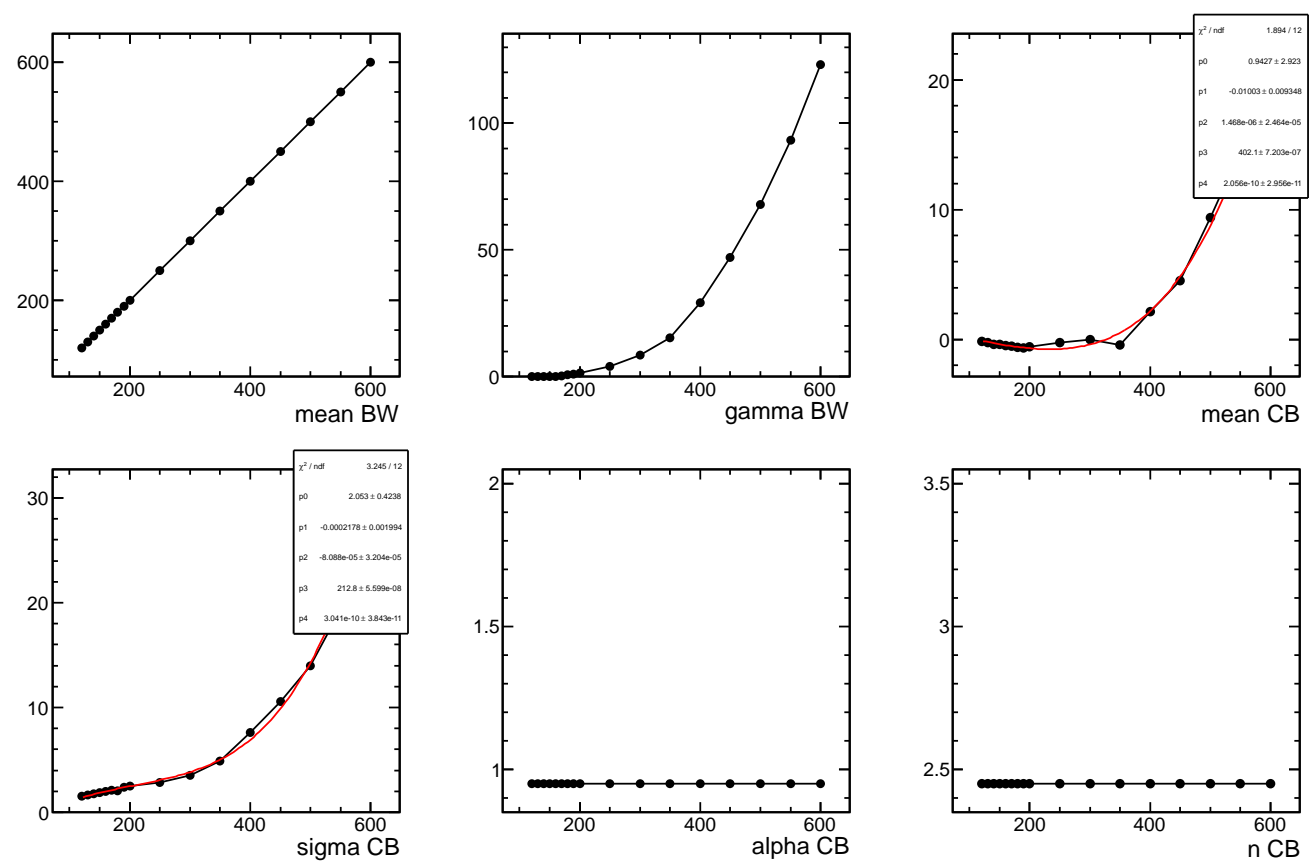

Figure 6.11: Breit-Wigner-like and Crystal Ball function parameters as a function of mass for the signal mass parameterization

To get the yields for the final signal contribution, we also need the overall signal efficiency such that the signal rates are given as:

$$
N=\sigma \times \mathcal{B R} \times \varepsilon
$$


CHAPTER 6. STUDIES OF RESONANCES IN $X \rightarrow Z Z \rightarrow 4 \ell$

The cross-section, $\sigma$, and branching ratios, $\mathcal{B R}$, are given in [92]. The signal efficiencies, which are also parameterized in $4 l$ invariant mass, are given in Fig. 6.12 for the $4 e$ (left), $4 \mu$ (middle) and $2 e 2 \mu$ (right) channels. Given the inputs for the signal and background contributions, we pull all the distributions together and also include the data. We observe $54 e$ events, $104 \mu$ events, and $62 e 2 \mu$ events in the CMS data sample of $1.66 \mathrm{fb}^{-1}$. This is shown in Fig. 6.13 with a Higgs mass hypothesis of $300 \mathrm{GeV}$ for illustration.
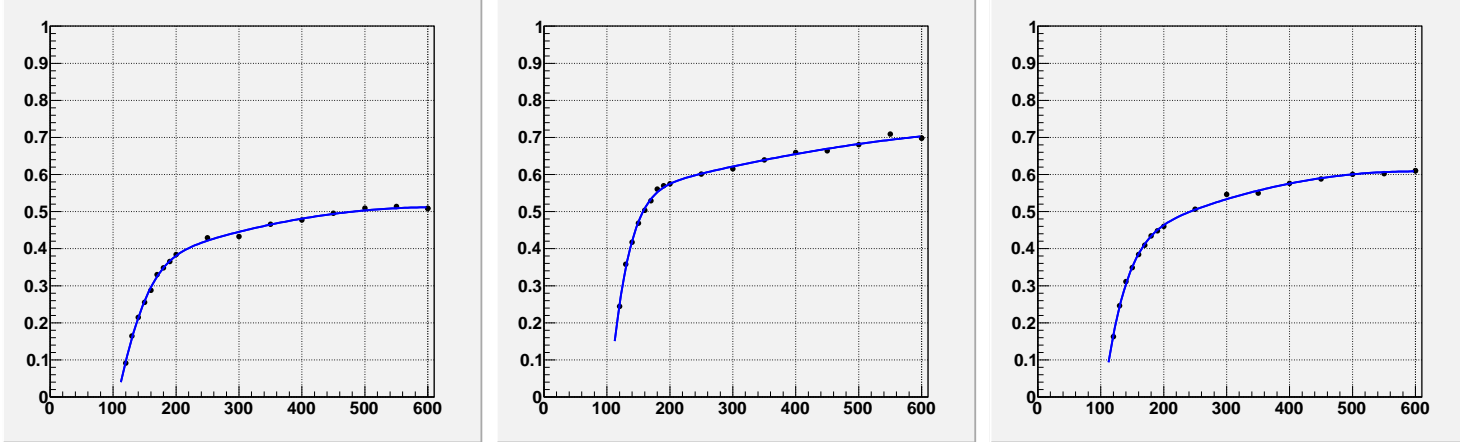

Figure 6.12: Signal efficiencies for the $4 e, 4 \mu$ and $2 e 2 \mu$ channels. 
CHAPTER 6. STUDIES OF RESONANCES IN $X \rightarrow Z Z \rightarrow 4 \ell$
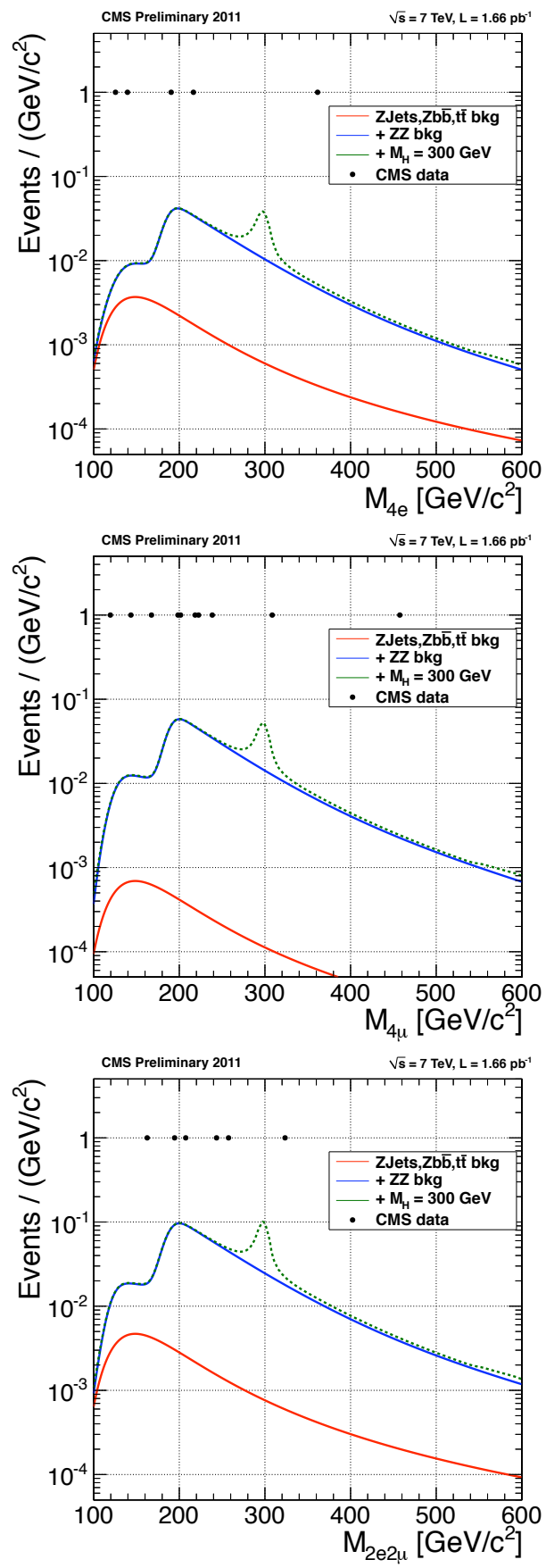

Figure 6.13: All input shapes are shown for signal (300 GeV) and background including data points 
CHAPTER 6. STUDIES OF RESONANCES IN $X \rightarrow Z Z \rightarrow 4 \ell$

\section{Results}

The observed and mean expected $95 \% \mathrm{CL}$ upper limits on Higgs $\sigma(p p \rightarrow \mathrm{H}+$ $X) \times \mathcal{B}(\mathrm{ZZ} \rightarrow 4 \ell)$ from an unbinned mass distribution analysis method, obtained for Higgs masses in the range 110-600 GeV are shown in Fig. 6.14. The left plot shows exclusion limits for the SM Higgs boson. The limits are made using a $\mathrm{CL}_{\mathrm{s}}$ approach, for the expected ratios to the SM. The right plot shows the excluded cross-section times branching ratio to $Z Z \rightarrow 4 l\left(\sigma_{H} \times B R(Z Z \rightarrow 4 l)\right)$. For comparison it shows the same quantity for the SM Higgs boson and a possible SM extension with a sequential fourth family of high mass fermions (SM4) [105-108]. The bands represent the $1 \sigma$ and $2 \sigma$ probability intervals around the expected limit. The expected limits reflect the dependence of the branching ratio $\mathcal{B}(H \rightarrow Z Z)$ on the Higgs boson mass. The worsening limits at high masses arise from the decreasing signal cross section. The reduced sensitivity around $m_{\mathrm{H}}=160 \mathrm{GeV}$ and for low masses arise from the very small $H \rightarrow Z Z$ branching ratio in these regions. By virtue of the excellent mass resolution and low background the structure in the measured limits follows the distribution of the observed events.

\section{Combination with other Higgs channels}

In this section so far, we have presented the latest search for the Higgs boson in the channel $H \rightarrow Z Z \rightarrow 4 l$. By itself, this decay mode nearly excludes much 
CHAPTER 6. STUDIES OF RESONANCES IN $X \rightarrow Z Z \rightarrow 4 \ell$

of the region just above the $Z Z$ invariant mass threshold. However, to get the most statistically powerful limit on the SM Higgs, we use the information from the $Z Z \rightarrow 4 l$ decay channel in combination with the other SM Higgs decay modes. The other SM Higgs decay modes considered by CMS in the most recent results are $H \rightarrow \gamma \gamma$ [109], $H \rightarrow \tau^{+} \tau^{-}$[110], $H \rightarrow b \bar{b}$ [111], $H \rightarrow W^{+} W^{-} \rightarrow 2 l 2 \nu$ [112], $H \rightarrow Z Z \rightarrow 4 l$ [83], $H \rightarrow Z Z \rightarrow 2 l 2 \tau$ [113], $H \rightarrow Z Z \rightarrow 2 l 2 q[39]$ and $H \rightarrow Z Z \rightarrow 2 l 2 \nu$ [114]. Discussion of each of the individual modes are left to the references. By doing a statistical combination of all these channels, we exclude at $95 \% \mathrm{CL}$ the SM Higgs mass in three mass ranges: $145-216,226-288$, and $310-400 \mathrm{GeV}$. The statistical combination is shown in Fig. 6.15. The top of Fig. 6.15 shows the latest limits from CMS along with constraints on the Higgs mass from LEP and the Tevatron. The bottom of Fig. 6.15 shows the same limits but with the individual contributions from each individual Higgs decay mode. The latest limits on the Higgs mass point to the regions where the SM Higgs mass may still be possible. In particular, these limits exclude the SM Higgs in a large region above the $Z Z$ mass threshold where the channel $Z Z \rightarrow 4 l$ has the most sensitivity. 
CHAPTER 6. STUDIES OF RESONANCES IN $X \rightarrow Z Z \rightarrow 4 \ell$
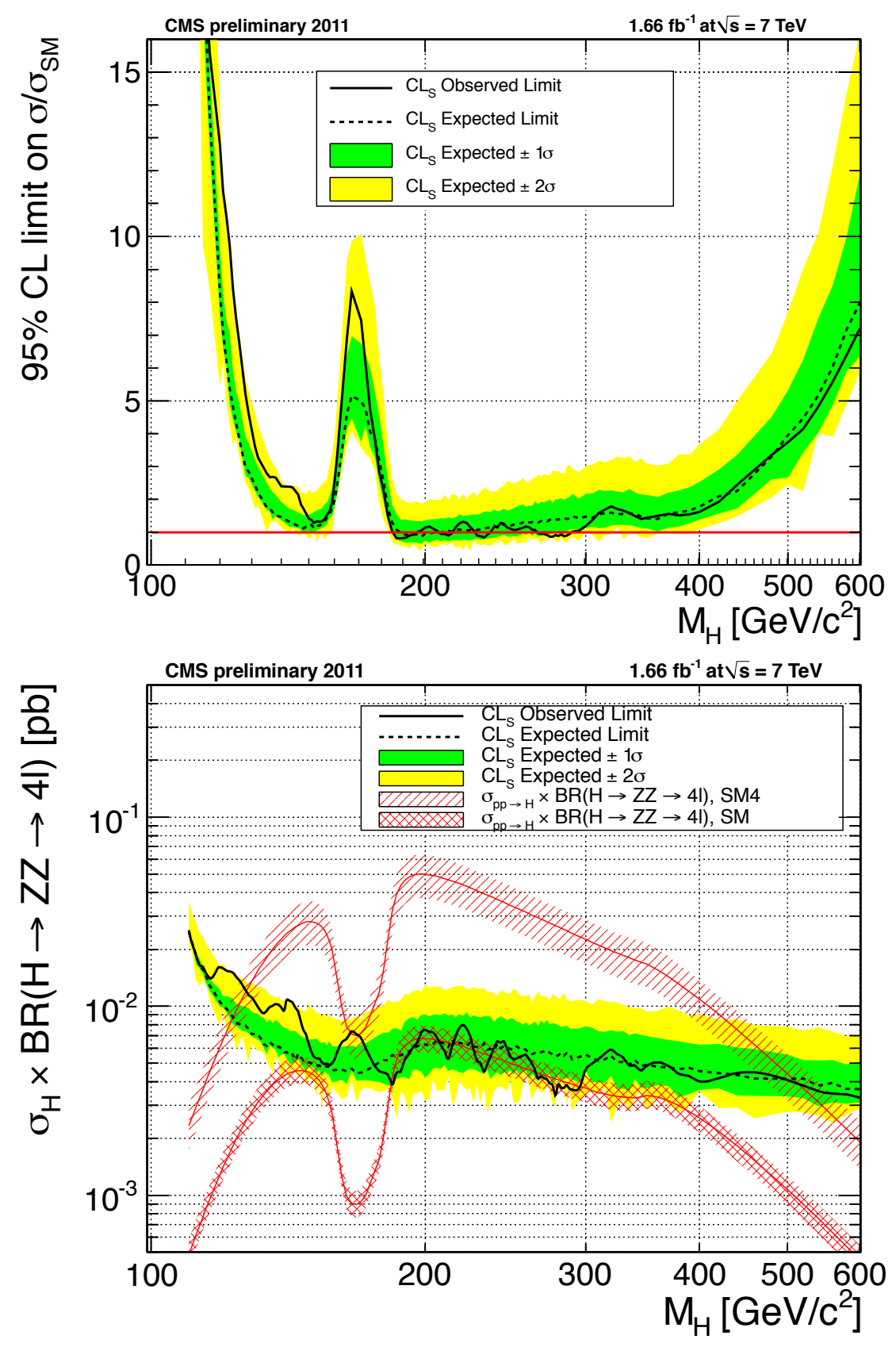

Figure 6.14: The mean expected and the observed upper limits at 95\% C.L. on $\sigma(p p \rightarrow$ $\mathrm{H}+\mathrm{X}) \times \mathcal{B}(\mathrm{ZZ} \rightarrow 4 \ell)$ 

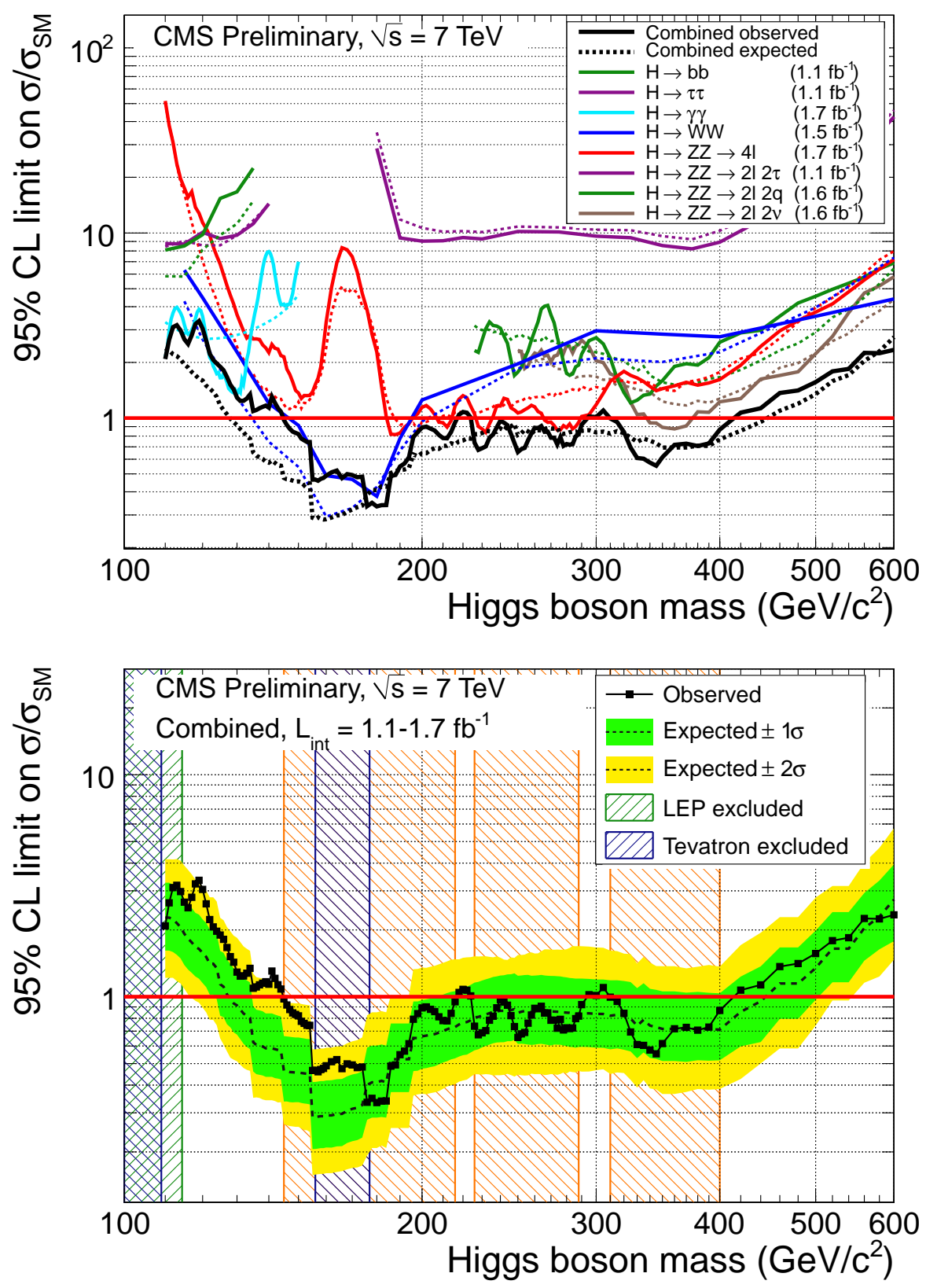

Figure 6.15: Combined CMS Higgs Limits with 1.1 to $1.7 \mathrm{fb}^{-1}$. 


\section{Chapter 7}

\section{Conclusion and Outlook}

"Fifteen hundred years ago everybody knew the Earth was the center of the universe. Five hundred years ago, everybody knew the Earth was flat, and fifteen minutes ago, you knew that humans were alone on this planet. Imagine what you'll know tomorrow."

- Kay, MIB

After nearly 20 years of preparation, the dawn of the LHC era has arrived. With a new energy regime available, physicists are able to probe the Standard Model and physics beyond it in an unprecedented way. The LHC has been performing well and providing data to the experiments at a current pace of approximately $1 \mathrm{fb}^{-1}$ a month when constantly running. CMS and the other LHC experiments are producing a large amount of physics results from exotic particle searches to constraints on supersymmetry to precision electroweak physics to searches for the Higgs boson. As the search for

physics beyond the SM continues, it becomes important to refine our techniques and methods such that we can extract as much information from our data as possible. 


\section{CHAPTER 7. CONCLUSION AND OUTLOOK}

In this thesis, we explore the general kinematics of resonances. We take advantage of spin correlations in particle interactions to understand the dynamics of resonance production and decay. We use a multivariate likelihood method to analyze the observables in an efficient way. Two applications of these methods are presented. In the first, the Drell-Yan process is analyzed and the weak mixing angle is measured. In the second, the channel, $X \rightarrow Z Z \rightarrow 4 \ell$, is examined in great detail. We derive general angular distributions and use them to separate different possible signal hypotheses and propose techniques for understanding the couplings of new resonances to the SM fields. However, the methods can be applied in many other ways. For example, it is being used in the study of the $Z Z \rightarrow 2 \ell 2 q$ final state [39] in the search for the Higgs. It can also be applied to the search for new gauge bosons decaying in a dilepton final state. The concept of understanding the full kinematics of a process is a general one which allows us to extract as much information as possible from a process on an event-by-event basis.

In the analysis of the weak mixing angle, we tested our formalism on the DrellYan process. We made a first measurement of $\sin ^{2} \theta_{W}$ at the LHC in proton-proton collisions and in the dimuon decay mode. The weak mixing angle is measured to be

$$
\sin ^{2} \theta_{W}=0.2287 \pm 0.0020 \text { (stat.) } \pm 0.0025 \text { (sys.) }
$$

The measurement is in agreement with those from other processes including the Tevatron, LEP and SLD. With the current data sample, the statistical and systematic errors are similar. However, to improve the measurement in the future, the systematic un- 


\section{CHAPTER 7. CONCLUSION AND OUTLOOK}

certainties must be reduced. While some systematic uncertainties should be reduced with a better understanding of the detector or improved simulation, an extension of the leading order model may be required.

In the analysis of $X \rightarrow Z Z \rightarrow 4 \ell$ resonances, we provide a generalized modelindependent formalism for describing the decay kinematics. In particular, we studied various signal hypotheses and statistically quantified how well we could distinguish different new physics models. From our analysis, we find that at the time of the discovery of a new resonance, it is already possible to make a statement about its spin and parity. With the current CMS data sample of approximately $1.66 \mathrm{fb}^{-1}$, we perform a search for the SM Higgs boson in the $p p \rightarrow H \rightarrow Z Z \rightarrow 4 \ell$ channel. No statistically significant deviation from expectation is observed. In the region $180<m_{H}<300 \mathrm{GeV}$, the SM Higgs boson is excluded at $95 \% \mathrm{CL}$ or nearly excluded. Combining the results of the $H \rightarrow Z Z \rightarrow 4 \ell$ channel with searches in other decay modes at CMS, the Higgs is excluded in three mass ranges: 145-216, 226-288, and 310-400 GeV.

In these two analyses, the measurement of $\sin ^{2} \theta_{W}$ and the search for the Higgs boson, we probe the nature of electroweak symmetry breaking in the SM in different ways. The weak mixing angle is a free parameter of the SM and its measurement confirms our description of the electroweak symmetry. The measurement of the weak mixing angle, along with measurements of the top quark, $W$ and $Z$ masses and others precision electroweak measurements, provide indirect constraints on the mass of the Higgs boson. In Fig. 1.2 from Chapter 1, the results of those indirect constraints on 


\section{CHAPTER 7. CONCLUSION AND OUTLOOK}

the Higgs mass as well as direct constraints from LEP and the Tevatron, previous to this work, were shown [4]. From our search for the Higgs boson at the LHC, the constraints on the Higgs mass continue are dramatically improved.

The LHC plans to provide at least double our current data sample by the end of 2011 and, before a technical stop at the end of 2012, the LHC plans to provide approximately $10 \mathrm{fb}^{-1}$ more data. This anticipated sample will be sufficient to exclude the SM Higgs boson in the full mass range from 110-600 GeV. If a SM Higgs boson exists, there should be hint in the coming year, but if it is excluded instead, this would require a shift in our understanding of how the electroweak symmetry is broken. It is a very exciting time for particle physics. The search for physics beyond the SM at the LHC continues... 


\section{Acknowledgements}

There are so very many people that I would like to thank in helping me get to this point. There is certainly not enough space here to do everyone justice though I hope that my profound appreciation is understood.

First, I would like to acknowledge my advisor, Andrei Gritsan. I am very grateful to have spent the last five years with him learning, studying, discussing, and everything in between. I appreciate most the time and care that he has put into our relationship which is certainly above and beyond what I had hoped for and expected. From our very first discussions of survey constraints in alignment to the long dissection of those gruesome angular distributions, I have learned so much. It goes beyond physics as well, from small things such as the ergonomic placement of my monitor to timing and planning for graduating and looking for a job. It goes without saying that I count myself very fortunate to have Andrei as my advisor.

I would also like to thank the rest of the JHU CMS group. Bruce, Barry, Petar, Morris and Sal have all provided valuable advice, insight, and collaboration over my graduate career. I thank Gavril for his lengthy discussions while carpooling. Working 


\section{ACKNOWLEDGEMENTS}

with Zijin was very educational; he was a joy to work with and I very much appreciate his patience. I am very grateful to have had the opportunity to meet and work with Alessio. From our "contentious" collaboration on alignment to our (in)famous Battle Gnocchi, I was very lucky to have the chance to learn from and laugh with him. I would like to thank the students of the group as well. I appreciate the chats with the "old guard" Jen and Mark and of course, Yanyan, for the countless amount of knowledge and experience she has passed on. I would like to thank LB for the many chats and his camaraderie; I am fortunate to have such a good friend. I am also fortunate to have been able to work with Andrew whose enthusiasm and sense of humor was always a joy. Finally I would like to acknowledge the younger students, especially Kevin, with whom I am lucky to have worked.

I would like to acknowledge all the great people in the JHU Physics and Astronomy department. I am fortunate to have had the opportunity to collaborate with Markus and Kirill. Their expertise and knowledge is vast and I am happy they were willing to spend some of their time with me to impart their wisdom. I am indebted to Keith for his discussion of Chapter 2, but more importantly, for his friendship from day one of graduate school. I would like to thank all the staff in Bloomberg as well from Lonnie, Mike and Mark to all the great people in the main office who made my time in Baltimore great fun and kept me from falling too far behind any administrative deadlines. Surely, I also cannot forget all the wonderful friends I have made in the department: Justin, Sarah, Stuart, Waqas and all the other wonderful people from my 


\section{ACKNOWLEDGEMENTS}

year; and Tom, Reenie, Seamus, Luke, and many many others. I appreciate the great times we have all spent together that have made graduate school such a memorable time and of course, the many amazing athletic triumphs.

I would also like to take the opportunity to acknowledge all of my collaborators on CMS. There are countless discussions and presentations that all have contributed to my education. In particular, I would like to thank the tracker alignment group whose members have always been so welcoming and easy to work with; and especially Roberto, with whom I am happy to have gotten to know well both in and outside of physics. I would also like to thank my colleagues in other groups with which I was involved, the Electroweak, Exotic, and Higgs as well as the Tracker DPG and Tracking and Muon POGs for their endless collaboration. Finally, I would like to thank the LHC and all the people who work so hard on it for providing the data to CMS and the other experiments.

Lastly, I would like to thank my family for all their support through the years. It has certainly been a long journey but without their unwavering love, it would not have been possible. I thank my mom and dad for always being proud of me no matter what, and I thank Tammy, Matt, and Mall-E for their support and to whom I am grateful to have gotten to know better during my time at JHU. I also would like to thank my new parents and sisters for their encouragement; their enthusiasm is very much recognized

and appreciated. Finally, I am grateful to my wonderful wife Cancan. Her support and love has always been my constant making the tough days easier to bear and the good 


\section{ACKNOWLEDGEMENTS}

days even better. I am lucky she is willing to put up with me and love me in spite of myself. 


\section{Bibliography}

[1] H. Murayama, "Quantum Universe," 2007, slides of a talk given January 22, 2007 at the University of Tennessee.

[2] F. O. W. T. S. Kretzer, H.L. Lai, "Cteq6 parton distributions with heavy quark mass effects," Phys. Rev., vol. D 69, p. 114005, 2004.

[3] L. Evans and P. Bryant (eds.), "LHC machine," JINST, vol. 3, p. S08001, 2008.

[4] T. Group, “Design Report Tevatron 1 project,” 1984.

[5] S. Weinberg, "A Model of Leptons," Phys. Rev. Lett., vol. 19, pp. 1264-1266, 1967.

[6] K. Nakamura et al., "Review of particle physics," J.Phys.G, vol. G37, p. 075021, 2010.

[7] R. Barate et al., "Search for the standard model Higgs boson at LEP," Phys.Lett., vol. B565, pp. 61-75, 2003. 


\section{BIBLIOGRAPHY}

[8] T. CDF, D. Collaborations, t. T. N. Phenomena, and H. W. Group, "Combined CDF and D0 Upper Limits on Standard Model Higgs Boson Production with up to $8.6 \mathrm{fb}-1$ of Data," 2011, " Temporary entry *.

[9] K. G. Begeman, A. H. Broeils, and R. H. Sanders, "Extended rotation curves of spiral galaxies: Dark haloes and modified dynamics," Mon. Not. Roy. Astron. Soc., vol. 249, p. 523, 1991.

[10] A. G. Riess et al., “Observational Evidence from Supernovae for an Accelerating Universe and a Cosmological Constant," Astron. J., vol. 116, pp. 1009-1038, 1998.

[11] A. Sakharov, "Violation of CP Invariance, c Asymmetry, and Baryon Asymmetry of the Universe," Pisma Zh.Eksp.Teor.Fiz., vol. 5, pp. 32-35, 1967, reprinted in "Kolb, E.W. (ed.), Turner, M.S. (ed.): The early universe" 371-373, and in "Lindley, D. (ed.) et al.: Cosmology and particle physics" 106-109, and in Sov. Phys. Usp. 34 (1991) 392-393 [Usp. Fiz. Nauk 161 (1991) No. 5 61-64].

[12] J. J. Sakurai and J. Napolitano, "Modern quantum physics," 2011.

[13] F. Halzen and A. D. Martin, "QUARKS AND LEPTONS: AN INTRODUCTORY COURSE IN MODERN PARTICLE PHYSICS," 1984.

[14] M. Jacob and G. Wick, "On the general theory of collisions for particles with spin," Annals Phys., vol. 7, pp. 404-428, 1959. 


\section{BIBLIOGRAPHY}

[15] F. Mandl and G. Shaw, "QUANTUM FIELD THEORY," 1985.

[16] K. Grotz and H. Klapdor, "The Weak interaction in nuclear, particle and astrophysics," 1990.

[17] E. Leader and E. Predazzi, "An Introduction to gauge theories and modern particle physics. Vol. 1: Electroweak interactions, the new particles and the parton model," Camb.Monogr.Part.Phys.Nucl.Phys.Cosmol., vol. 3, p. 1, 1996.

[18] E. Bruning, Oliver S., E. Collier, P., E. Lebrun, P., E. Myers, S., E. Ostojic, R. et al., "LHC Design Report. 1. The LHC Main Ring," 2004.

[19] G. Bayatian et al., "CMS physics: Technical design report," 2006.

[20] CMS Collaboration, "Commissioning of the CMS High-Level Trigger with Cosmic Rays," JINST, vol. 5, p. T03005, 2010.

[21] CMS Collaboration, "Commissioning and Performance of the CMS Pixel Tracker with Cosmic Ray Muons," JINST, vol. 5, p. T03007, 2010.

[22] CMS Collaboration, "Commissioning and Performance of the CMS Silicon Strip Tracker with Cosmic Ray Muons," JINST, vol. 5, p. T03008, 2010.

[23] CMS Collaboration, "Precise Mapping of the Magnetic Field in the CMS Barrel Yoke using Cosmic Rays," JINST, vol. 5, p. T03021, 2010.

[24] CMS Collaboration, "Commissioning of the CMS Experiment and the Cosmic Run at Four Tesla," JINST, vol. 5, p. T03001, 2010. 


\section{BIBLIOGRAPHY}

[25] CMS Collaboration, "Alignment of the CMS Silicon Tracker during Commissioning with Cosmic Rays," JINST, vol. 5, p. T03009, 2010.

[26] V. Blobel, "Software alignment for tracking detectors," Nucl.Instrum.Meth., vol. A566, pp. 5-13, 2006.

[27] V. Karimaki, T. Lampen, and F. Schilling, "The HIP algorithm for track based alignment and its application to the CMS pixel detector," 2006.

[28] D. Brown, A. Gritsan, Z. Guo, and D. Roberts, "Local Alignment of the BABAR Silicon Vertex Tracking Detector," Nucl.Instrum.Meth., vol. A603, pp. 467-484, 2009.

[29] W. Adam et al., "Stand-alone Cosmic Muon Reconstruction Before Installation of the CMS Silicon Strip Tracker," JINST, vol. 4, p. P05004, 2009.

[30] W.-Y. Keung, I. Low, and J. Shu, "Landau-Yang Theorem and Decays of a Z' Boson into Two Z Bosons," Phys.Rev.Lett., vol. 101, p. 091802, 2008.

[31] L. Randall and R. Sundrum, "A Large mass hierarchy from a small extra dimension," Phys.Rev.Lett., vol. 83, pp. 3370-3373, 1999.

[32] K. Agashe, H. Davoudiasl, G. Perez, and A. Soni, "Warped Gravitons at the LHC and Beyond," Phys.Rev., vol. D76, p. 036006, 2007.

[33] M. J. Strassler and K. M. Zurek, "Echoes of a hidden valley at hadron colliders," Phys.Lett., vol. B651, pp. 374-379, 2007. 


\section{BIBLIOGRAPHY}

[34] Y. Gao, A. V. Gritsan, Z. Guo, K. Melnikov, M. Schulze et al., "Spin determination of single-produced resonances at hadron colliders," Phys.Rev., vol. D81, p. 075022, 2010.

[35] J. M. Campbell, J. Huston, and W. Stirling, "Hard Interactions of Quarks and Gluons: A Primer for LHC Physics," Rept.Prog.Phys., vol. 70, p. 89, 2007.

[36] C.-N. Yang, "Selection Rules for the Dematerialization of a Particle Into Two Photons," Phys. Rev., vol. 77, pp. 242-245, 1950.

[37] L. D. Landau, Dokl. Akad. Nawk, vol. 60, pp. 207-209, 1948.

[38] T. Trueman, "phi phi DECAY AS A PARITY AND SIGNATURE TEST," Phys.Rev., vol. D18, p. 3423, 1978.

[39] J. R. Dell'Aquila and C. A. Nelson, "P OR CP DETERMINATION BY SEQUENTIAL DECAYS: V1 V2 MODES WITH DECAYS INTO anti-lepton (A) lepton (B) AND/OR anti-q (A) q (B)," Phys.Rev., vol. D33, p. 80, 1986.

[40] CMS Collaboration, "Search for the standard model Higgs Boson in the decay channel $\mathrm{H} \rightarrow \mathrm{ZZ} \rightarrow \ell^{-} \ell^{+} \mathrm{q} \overline{\mathrm{q}}$ at CMS," CMS PAS, vol. HIG-11-017, 2011.

[41] J. L. Rosner, "Forward - backward asymmetries in hadronically produced lepton pairs," Phys.Rev., vol. D54, pp. 1078-1082, 1996.

[42] S. Choi, . Miller, D.J., M. Muhlleitner, and P. Zerwas, "Identifying the Higgs spin and parity in decays to Z pairs," Phys.Lett., vol. B553, pp. 61-71, 2003. 


\section{BIBLIOGRAPHY}

[43] S. Drell and T.-M. Yan, "Massive Lepton Pair Production in Hadron-Hadron Collisions at High-Energies," Phys.Rev.Lett., vol. 25, pp. 316-320, 1970.

[44] J. Rosner, "Off-peak lepton asymmetries from new z's," Phys. Rev. D, vol. 35, p. 2244, 1987.

[45] M. Dittmar, "Neutral current interference in the tev region: The experimental sensitivity at the cern lhc," Phys. Rev. D, vol. 55, pp. 161-166, 1997.

[46] D. H., H. J.L., and R. T.G., "Phenomenology of the randall-sundrum gauge hierarchy model," Phys. Rev. Lett., vol. 84, pp. 2080-2083, 2000.

[47] B. Allanach, K. Odagiri, M. A. Parker, and B. Webber, "Searching for narrow graviton resonances with the ATLAS detector at the Large Hadron Collider," JHEP, vol. 0009, p. 019, 2000.

[48] B. Allanach, K. Odagiri, M. Palmer, M. A. Parker, A. Sabetfakhri et al., “Exploring small extra dimensions at the large hadron collider," JHEP, vol. 0212, p. 039, 2002.

[49] R. Cousins, J. Mumford, and V. Valuev, "Measurement of forward-backward asymmetry of simulated and reconstructed $z^{\prime} \rightarrow \mu^{+} \mu^{-}$events in cms," CMS Note, vol. 2005/022, 2005.

[50] P. Osland, A. Pankov, N. Paver, and A. Tsytrinov, "Spin identification of the 


\section{BIBLIOGRAPHY}

Randall-Sundrum resonance in lepton-pair production at the LHC," Phys.Rev., vol. D78, p. 035008, 2008.

[51] "Precision electroweak measurements on the $Z$ resonance," Phys.Rept., vol. 427, pp. 257-454, 2006.

[52] G. P. Zeller et al., "A precise determination of electroweak parameters in neutrino nucleon scattering," Phys. Rev. Lett., vol. 88, p. 091802, 2002.

[53] and V. Abazov, "Measurement of $\sin ^{2} \theta_{\text {eff }}^{\text {lept }}$ and $Z$-light quark couplings using the forward-backward charge asymmetry in $p \bar{p} \rightarrow Z / \gamma^{*} \rightarrow e^{+} e^{-}$events with $\mathrm{L}=5.0$ $\mathrm{fb}^{-1}$ at $\sqrt{s}=1.96 \mathrm{TeV}, " 2011$.

[54] K. Nakamura et al., “Particle Data Group,” J. Phys. G, vol. 37, p. 075021, 2010.

[55] J. Collins and D. Soper, "Angular distribution of dileptons in high-energy hadron collisions," Phys. Rev. D, vol. 16, p. 2219, 1977.

[56] H.-L. Lai, M. Guzzi, J. Huston, Z. Li, P. Nadolsky, J. Pumplin, and C.-P. Yuan, “New parton distributions for collider physics," 2010.

[57] A. D. Martin, W. J. Stirling, R. S. Thorne, and G. Watt, "Parton distributions for the LHC," Eur. Phys. J., vol. C63, pp. 189-285, 2009.

[58] R. D. Ball et al., "A first unbiased global NLO determination of parton distributions and their uncertainties," Nucl. Phys., vol. B838, pp. 136-206, 2010. 


\section{BIBLIOGRAPHY}

[59] A. De Roeck and R. S. Thorne, "Structure Functions," 2011.

[60] M. Botje et al., "The PDF4LHC Working Group Interim Recommendations," 2011.

[61] T. Sjostrand, S. Mrenna, and P. Skands, "Pythia 6.4 physics and manual," JHEP, vol. 05, p. 026, 2006.

[62] "The cms experiment at the cern lhc," JINST, vol. 0803, p. S08004, 2008.

[63] P. Nason, "A new method for combining nlo qcd with shower monte carlo algorithms," JHEP, vol. 11, p. 040, 2004.

[64] S. Frixione, P. Nason, and C. Oleari, "Matching nlo qcd computations with parton shower simulations: the powheg method," JHEP, vol. 11, p. 070, 2007.

[65] S. Alioli, P. Nason, C. Oleari, and E. Re, "Nlo vector-boson production matched with shower in powheg," JHEP, vol. 07, p. 06, 2008.

[66] J. Alwall, P. Demin, S. de Visscher, R. Frederix, M. Herquet, F. Maltoni, T. Plehn, D. Rainwater, and T. Stelzer, "Madgraph/madevent v4: The new web generation," JHEP, vol. 09, p. 028, 2007.

[67] N. Davidson, G. Navana, T. Przedzinski, E. Richter-Was, and Z. Was, "Universal interface of tauola technical and physics documentation," 2010.

[68] J. Sulkimo, M. Takahata, S. Tanaka, E. Tcherniaev, E. Safai-Tehrani, M. Tropeano, P. Truscott, H. Uno, P. Urban, M. Verderi, A. Walkden, W. Wander, H. Weber, 


\section{BIBLIOGRAPHY}

J. Wellisch, T. Wenaus, D. Williams, T. Yamada, H. Yoshida, and D. Zschiesche, "Geant 4 - a simulation toolkit," NIM A, vol. 506/3, pp. 250-303, 2003.

[69] J. Allison, K. Amako, J. Apostolakis, H. Araujo, P. Dubois, M. Asai, G. Barrand, R. Capra, S. Chauvie, R. Chytracek, G. Cirrone, G. Cooperman, G. Cosmo, G. Cuttone, G. Daquino, M. Donszelmann, M. Dressel, G. Folger, F. Foppiano, J. Generowicz, V. Grichine, S. Guatelli, P. Gumplinger, A. Heikkinen, I. Hrivnacova, A. Howard, S. Incerti, V. Ivanchenko, T. Johnson, F. Jones, T. Koi, R. Kokoulin, M. Kossov, H. Kurashige, V. Lara, S. Larsson, F. Lei, O. Link, F. Longo, M. Maire, A. Mantero, B. Mascialino, I. McLaren, P. Lorenzo, K. Minamimoto, K. Murakami, P. Nieminen, L. Pandola, S. Parlati, L. Peralta, J. Perl, A. Pfeiffer, M. Pia, A. Ribon, P. Rodrigues, G. Russo, S. Sadilov, G. Santin, T. Sasaki, D. Smith, N. Starkov, S. Tanaka, E. Tcherniaev, B. Tome, A. Trindade, P. Truscott, L. Urban, M. Verderi, A. Walkden, J. Wellisch, D. Williams, D. Wright, and $\mathrm{H}$. Yoshida, "Geant4 developments and applications," IEEE Transactions on Nuclear Science, vol. 53/1, pp. 270-278, 2006.

[70] F. James and M. Roos, "Minuit: A System for Function Minimization and Analysis of the Parameter Errors and Correlations," Comput.Phys.Commun., vol. 10, pp. 343-367, 1975.

[71] A. B. Arbuzov et al., "ZFITTER: A semi-analytical program for fermion pair 


\section{BIBLIOGRAPHY}

production in e+ e- annihilation, from version 6.21 to version 6.42," Comput. Phys. Commun., vol. 174, pp. 728-758, 2006.

[72] S. Alekhin, S. Alioli, R. D. Ball, V. Bertone, J. Blumlein et al., "The PDF4LHC Working Group Interim Report," 2011, * Temporary entry *.

[73] M. Botje, J. Butterworth, A. Cooper-Sarkar, A. de Roeck, J. Feltesse et al., "The PDF4LHC Working Group Interim Recommendations," 2011.

[74] M. Whalley, D. Bourilkov, and R. Group, "The Les Houches accord PDFs (LHAPDF) and LHAGLUE," 2005.

[75] C. Carloni Calame, G. Montagna, O. Nicrosini, and A. Vicini, "Precision electroweak calculation of the production of a high transverse-momentum lepton pair at hadron colliders," JHEP, vol. 0710, p. 109, 2007.

[76] P. Golonka and Z. Was, "PHOTOS Monte Carlo: A Precision tool for QED corrections in $Z$ and $W$ decays," Eur.Phys.J., vol. C45, pp. 97-107, 2006.

[77] "Precision electroweak measurements on the Z resonance," Phys. Rept., vol. 427, pp. 257-454, 2006.

[78] D. e. a. Acosta, "Measurement of the forward-backward charge asymmetry of electron-positron pairs in $p \bar{p}$ collisions at $\sqrt{s}=1.96$ tev," Phys. Rev. D, vol. 71, no. 5, p. 052002, Mar 2005. 


\section{BIBLIOGRAPHY}

[79] V. M. e. a. Abazov, "Measurement of the forward-backward charge asymmetry and extraction of $\sin ^{2} \theta_{W}^{e f f}$ in $p \bar{p} \rightarrow z / \gamma^{*}+x \rightarrow e^{+} e^{-}+x$ events produced at $\sqrt{s}=1.96$ tev," Phys. Rev. Lett., vol. 101, no. 19, p. 191801, Nov 2008.

[80] A. Aktas et al., "A determination of electroweak parameters at HERA," Phys. Lett., vol. B632, pp. 35-42, 2006.

[81] K. Hagiwara, J. Kanzaki, Q. Li, and K. Mawatari, "HELAS and MadGraph/MadEvent with spin-2 particles," Eur.Phys.J., vol. C56, pp. 435-447, 2008.

[82] J. Alwall, A. Ballestrero, P. Bartalini, S. Belov, E. Boos et al., "A Standard format for Les Houches event files," Comput.Phys.Commun., vol. 176, pp. 300-304, 2007.

[83] CMS Collaboration, "Search for a Standard Model Higgs boson in the decay channel $H \rightarrow Z Z^{(*)} \rightarrow 4 \ell, "$ CMS PAS, vol. HIG-11-015, 2011.

[84] R. Cousins, J. Mumford, J. Tucker, and V. Valuev, "Spin discrimination of new heavy resonances at the LHC," JHEP, vol. 0511, p. 046, 2005.

[85] A. Fitzpatrick, J. Kaplan, L. Randall, and L.-T. Wang, "Searching for the KaluzaKlein Graviton in Bulk RS Models," JHEP, vol. 0709, p. 013, 2007.

[86] O. Antipin, D. Atwood, and A. Soni, "Search for RS gravitons via W(L)W(L) decays," Phys.Lett., vol. B666, pp. 155-161, 2008.

[87] T. Aaltonen et al., "Combined CDF and D0 Upper Limits on Standard Model Higgs Boson Production with up to $8.2 \mathrm{fb}^{-1}$ of Data," 2011. 


\section{BIBLIOGRAPHY}

[88] "Precision electroweak measurements and constraints on the Standard Model," 2010.

[89] B. W. Lee, C. Quigg, and H. Thacker, “Weak Interactions at Very High-Energies: The Role of the Higgs Boson Mass," Phys.Rev., vol. D16, p. 1519, 1977.

[90] L. Durand, J. M. Johnson, and J. L. Lopez, "Perturbative unitarity revisited: a new upper bound on the Higgs boson mass," Phys.Rev.Lett., vol. 64, p. 1215, 1990.

[91] K. Riesselmann, "Limitations of a standard model Higgs boson," 1997.

[92] LHC Higgs Cross Section Working Group, S. Dittmaier, C. Mariotti, G. Passarino, and R. Tanaka (Eds.), "Handbook of LHC Higgs Cross Sections: 1. Inclusive Observables," CERN-2011-002, CERN, Geneva, 2011.

[93] S. M. T. Sjostrand and P. Z. Skands, "Pythia 6.4 physics and manual," JHEP, vol. 0605, 2006.

[94] "Geant."

[95] S. Alioli, P. Nason, C. Oleari and E. Re, "A general framework for implementing nlo calculations in shower monte carlo programs: the powheg box," JHEP.

[96] A. Bredenstein, A. Denner, S. Dittmaier, and M. M. Weber, "Precise predictions for the Higgs-boson decay $\mathrm{H} \rightarrow \mathrm{WW} / \mathrm{ZZ} \rightarrow 4$ leptons," Phys. Rev., vol. D74, p. 013004, 2006. 


\section{BIBLIOGRAPHY}

[97] A. Bredenstein, A. Denner, S. Dittmaier, and M. Weber, "Radiative corrections to the semileptonic and hadronic Higgs-boson decays $\mathrm{H} \rightarrow \mathrm{W}$ W / Z Z $\rightarrow 4$ fermions," JHEP, vol. 0702, p. 080, 2007.

[98] A. Djouadi, J. Kalinowski, M. Muhlleitner, and M. Spira, "An update of the program HDECAY," in The Les Houches 2009 workshop on TeV colliders: The tools and Monte Carlo working group summary report, 2010.

[99] S. Actis, G. Passarino, C. Sturm, and S. Uccirati, "NNLO Computational Techniques: the Cases $H \rightarrow \gamma \gamma$ and $H \rightarrow g g, "$ Nucl. Phys., vol. B811, pp. 182-273, 2009.

[100] J. Campbell and R. K. Ellis, Nucl. Phys. Proc. Suppl., vol. 205-206, pp. 10-15, 2010, mCFM code: http://mcfm.fnal.gov/.

[101] J. Alwall et al., "MadGraph/MadEvent v4: The New Web Generation," JHEP, vol. 09, p. 028, 2007.

[102] A.L. Read, "First workshop on confidence limits," CERN Report 2000-005 p. 81, 2000.

[103] S. e. a. Baffioni, "Search strategy for the higgs boson in the $z z^{*}$ decay channel with the cms experiment," CMS Note, vol. 2008/050, 2008.

[104] P. Avery, M. Chen, A. Drozdetskiy, A. Korytov, G. Mitselmakher, and M. Snow- 


\section{BIBLIOGRAPHY}

ball, "A complete model of the four-lepton invariant mass distributions for $h \rightarrow z z \rightarrow 4 \ell$ and $z z \rightarrow 4 \ell$ events," CMS Note, vol. 2011/202, 2011.

[105] Q. Li, M. Spira, J. Gao, and C. S. Li, “Higgs Boson Production via Gluon Fusion in the Standard Model with four Generations," Phys. Rev., vol. D83, p. 094018, 2011.

[106] C. Anastasiou, S. Buehler, F. Herzog, and A. Lazopoulos, "Total cross-section for Higgs boson hadroproduction with anomalous Standard Model interactions," 2011.

[107] N. B. Schmidt, S. A. Cetin, S. Istin, and S. Sultansoy, "The Fourth Standart Model Family and the Competition in Standart Model Higgs Boson Search at Tevatron and LHC," Eur. Phys. J., vol. C66, pp. 119-126, 2010.

[108] LHC Higgs Cross Section Working Group, "Fourth Generation Model," 2011, https://twiki.cern.ch/twiki/bin/view/LHCPhysics/SM4At7TeV.

[109] CMS Collaboration, "Search for a Higgs boson decaying into two photons in the CMS detector," CMS PAS, vol. HIG-11-021, 2011.

[110] CMS Collaboration, "Search for Neutral Higgs Bosons Decaying to Tau Pairs in pp Collisions at $\sqrt{s}=7 \mathrm{TeV}, "$ CMS PAS, vol. HIG-11-009, 2011.

[111] CMS Collaboration, "Search for the Standard Model Higgs Boson decaying to 


\section{BIBLIOGRAPHY}

Bottom Quarks and Produced in Association with a W or a Z Boson," CMS PAS, vol. HIG-11-012, 2011.

[112] CMS Collaboration, "Search for the Higgs Boson Decaying to $W^{+} W^{-}$in the Fully Leptonic Final State," CMS PAS, vol. HIG-11-014, 2011.

[113] CMS Collaboration, "Search for a Standard Model Higgs boson produced in the decay channel $H \rightarrow Z Z \rightarrow 2 \ell 2 \tau$ with CMS detector at $\sqrt{s}=7 \mathrm{TeV}$," CMS PAS, vol. HIG-11-013, 2011.

[114] CMS Collaboration, "Search for the Higgs boson in the $H \rightarrow Z Z \rightarrow 2 \ell 2 \nu$ channel in pp collisions at $\sqrt{s}=7$ TeV," CMS PAS, vol. HIG-11-016, 2011. 


\section{CURRICULUM VITAE}

\section{Nhan V. Tran}

Johns Hopkins University, Department of Physics and Astronomy

Email: ntran@pha.jhu.edu

Phone: $630-840-8202$

\section{Education}

Doctoral Candidate for Ph.D., Physics

Johns Hopkins University, September 2005 - present

B.S., Physics

Princeton University, September 2001 - June 2005

\section{Research Experience}

- Graduate Research, Johns Hopkins University, CMS Collaboration, 2006 - present

- Senior Thesis, Princeton University, "Preliminary Measurements Towards Detailed State-to-State Collisional Population Transfer in Excited Atoms", Spring 2005

- Summer Researcher, Princeton University - Summer 2004, Advisor: Michael Romalis; Summer 2002 \& 2003, Advisor: Daniel Marlow

- Junior Paper, Princeton University, "Richness-Dependent Cluster Correlation Functions From SDSS Data", Spring 2004

- Junior Paper, Princeton University, "CP Violation in Belle and the measurement of $\sin 2 \phi_{1}$ in $B^{0} \rightarrow \phi K_{s}^{0 \prime \prime}$, Fall 2003 


\section{CURRICULUM VITAE}

\section{Teaching Experience}

- Teaching Assistant: General Physics II, Spring 2006

- Teaching Assistant, General Physics I, Fall 2005

\section{Awards \& Grants}

NSF US LHC Graduate Student Support Award, 2009

URA Visiting Scholar, Fall 2009

\section{Presentations and Talks}

"Analysis of $Z \rightarrow l^{+} l^{-}$polarization at CMS", LHC students' poster session at next LHCC. Geneva, Switzerland, March 2011.

"Analysis of $Z \rightarrow l^{+} l^{-}$polarization at CMS", Recontres de Moriond, EW. La Thuile, Italy, March 2011.

"Model-independent spin and coupling determination of Higgs-like resonances", Higgs Hunting Workshop. Orsay, France, July 2010.

"Spin determination of single-produced resonances at the LHC", ICHEP 2010. Paris, France, July 2010.

"Spin determination of single-produced resonances at the LHC", Fermilab Users' Meeting. Batavia, IL, USA, June 2010. 


\section{CURRICULUM VITAE}

"Spin determination of single-produced resonances at the LHC", Pheno 2010 Symposium. Madison, WI, USA, May 2010.

"Spin determination of single-produced resonances at the LHC", Northwestern University High Energy Physics Seminar. Evanston, IL, USA, April 2010.

"CMS Alignment Implications for Physics Performance", LHC Detector Alignment Workshop. Geneva, Switzerland, June 2009.

"CMS Tracker Alignment and Implications on Physics Performance", Physics at the LHC Conference. Split, Croatia, October 2008.

\section{Publications}

- CMS Collaboration, "Search for a Standard Model Higgs boson in the decay channel $H \rightarrow Z Z^{(*)} \rightarrow 4 l^{\prime \prime}$, CMS PAS HIG-11-015 (2011)

- CMS Collaboration, "Measurement of the weak mixing angle in the Drell-Yan process at LHC with CMS", CMS PAS EWK-11-003 (2011).

- CMS Collaboration, "Measurement of Forward-Backward Asymmetry of Lepton Pairs and the Weak-mixing Angle at CMS", CMS PAS EWK-10-011 (2011).

- Y. Gao et al., "Spin determination of single-produced resonances at hadron colliders", Phys. Rev. D. 81, 075022 (2010).

- CMS Collaboration, "Alignment of the CMS silicon tracker during commissioning with cosmic rays", 2010 JINST 5 T03009.

- CMS Collaboration, "Commissioning and performance of the CMS pixel tracker with 


\section{CURRICULUM VITAE}

cosmic ray muons", 2010 JINST 5 T03007.

- CMS Collaboration, "Commissioning and performance of the CMS silicon strip tracker with cosmic ray muons", 2010 JINST 5 T03008.

- CMS Collaboration, "Performance of CMS muon reconstruction in cosmic-ray events", 2010 JINST 5 T03022.

- W. Adam et al., "CMS Tracker Alignment at the Integration Facility", JINST 4 T07001, 2009.

- A. Bonato, A.V. Gritsan, Z.J. Guo, N.V. Tran, E. Yazgan, "Angular Analysis of $Z \rightarrow l^{+} l^{-}$ and the Measurement of $\sin ^{2} \theta_{W}, "$ CMS AN-2011/031.

- N. Akchurin et al., "Measurement of the Forward-Backward Asymmetry of $\mu^{+} \mu^{-}$Pairs in CMS at $\sqrt{s}=7 \mathrm{TeV}$," CMS AN-2010/455.

- A. Bonato, A.V. Gritsan, Z.J. Guo, N.V. Tran, A. Whitbeck, "Angular Analysis of Resonances $p p \rightarrow X \rightarrow Z Z, "$ CMS AN-2010/351.

- A. Bonato et al., "Application of Survey Measurements in Tracker Alignment", CMS IN 2009/027.

- A.V. Gritsan, M. Kubantsev, N.V. Tran, "Optical Survey Analysis of the CMS Forward Pixel Detector and Application to Alignment with Tracks", CMS IN 2007/012.

\section{Outreach}

"The Science of the Large Hadron Collider", USA Science and Engineering Festival, Washington D.C., USA, October 2010. 


\section{CURRICULUM VITAE}

"Particle Physics, The Mysteries of the Universe, and the LHC", Central Bucks West High School, Doylestown, PA, USA, December 2009.

Johns Hopkins Physics Fair, Baltimore, MD, USA, April 2006 and April 2007.

\section{Extracurricular}

- Vice President, JHU Physics and Astromony Graduate Students (PAGS) 2006-2007

December 13, 2011 